\title{
THE STUDY AND IMPLEMENTATION OF ELECTRICALLY SMALL PRINTED ANTENNAS FOR AN INTEGRATED TRANSCEIVER DESIGN
}

\begin{abstract}
By
Peter Speer

Submitted to the graduate degree program in Electrical Engineering and Computer Science and the Graduate Faculty of the University of Kansas in partial fulfillment of requirements for the degree of Master of Science.
\end{abstract}

Chairperson

Date Defended: April 28, 2009 
The thesis Committee for Peter Speer certifies

that this is the approved version of the following thesis

\section{THE STUDY AND IMPLEMENTATION OF ELECTRICALLY SMALL PRINTED ANTENNAS FOR AN INTEGRATED TRANSCEIVER DESIGN}

Chairperson

Date approved: 


\section{Acknowledgments:}

It gives me great pleasure to honor those people who have been a positive influence and encouraged me in the completion of this project. I would like to thank my friends Jeremy Baker, Tony Cecil, Bruce Dawson, and Mike Landis for offering their excellent professional contributions and collaboration. They have graciously provided their time and energy in support this of work while being extremely busy with other work-related projects. Their helpful comments and excellent constructive feedback have provided a solid foundation for screening new ideas. I recognize them because they have always been there to help out and have generously given their time without hesitation.

I would like to foremost thank Dr. Allen for encouraging me to pursue this topic and return to school at the University of Kansas. He has provided me with excellent academic advice and outstanding support since the very beginning of my student career. I would like also to thank Dr. Stiles and Dr. Blunt for sitting on my committee and teaching me the necessary background material needed for success. They have been tremendously helpful along the way and I am deeply grateful to them. I would also like to thank the EECS department and faculty for its devout dedication while offering remote classes to working professionals.

I would also like to thank my wonderful wife Lauren who has been incredibly patient while I have committed time to this project. She has encouraged me to keep

going in tough times and has stood by me with optimism. Her support has been immensely helpful in the completion of this project and is extremely appreciated. To my family who has always supported me in life and in everything I have done, I am truly grateful for their positive influence and helpful encouragement. 


\section{Table of Contents:}

\begin{tabular}{|c|c|}
\hline & ............................. \\
\hline Intr & oduction .... \\
\hline 1.1 & Motivation. \\
\hline 1.2 & Overview... \\
\hline 1.3 & Applications . \\
\hline 1.4 & Definitions... \\
\hline 1.5 & Acronyms .................. \\
\hline Ante & enna Theory............... \\
\hline 2.1 & Performance .......... \\
\hline 2.1 .1 & General Data . \\
\hline 2.1.2 & Electrical Specifications.. \\
\hline 2.2 & Grounded Coplanar Waveguide Feed... \\
\hline 2.3 & Microstrip Width-to-Depth Ratio................ \\
\hline 2.4 & Microstrip Characteristic Impedance ........ \\
\hline 2.5 & Microstrip Effective Dielectric Constant. \\
\hline 2.6 & 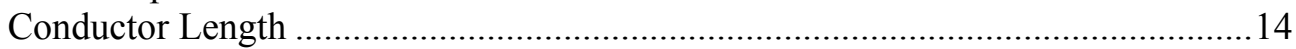 \\
\hline 2.7 & Geometries ............. \\
\hline 2.8 & Electrically Small Antennas.. \\
\hline Anso & oft Simulation..... \\
\hline 3.1 & Models (Top View)... \\
\hline 3.2 & Models (Isometric View). \\
\hline 3.3 & Inverted-F Antenna Simulation.. \\
\hline 3.3.1 & Antenna Orientation and Matching Circuit. . \\
\hline 3.3.2 & Simulated 3D Radiation Pattern Gain $(\mathrm{dB})$ after Matching ... \\
\hline 3.3 .3 & Simulated 2D Radiation Pattern after Matching ................... \\
\hline 3.3 .4 & 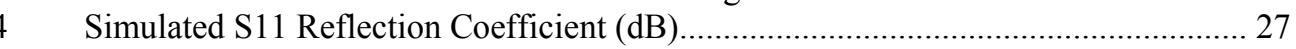 \\
\hline 3.3 .5 & Simulated Voltage Standing Wave Ratio (VSWR) ....................... \\
\hline 3.4 & Long Meandering Monopole Simulation ................................ \\
\hline 3.4 .1 & Antenna Orientation and Matching Circuit.. \\
\hline 3.4 .2 & 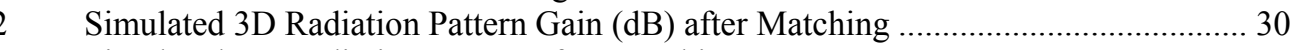 \\
\hline 3.4 .3 & 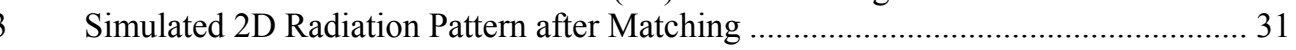 \\
\hline 3.4 .4 & Simulated S11 Reflection Coefficient $(\mathrm{dB}) \ldots \ldots$. \\
\hline 3.4 .5 & Simulated Voltage Standing Wave Ratio (VSWR).................. \\
\hline 3.5 & Short Meandering-Monopole Simulation ……........................ \\
\hline 3.5 .1 & Antenna Orientation and Matching Circuit \\
\hline 3.5 .2 & 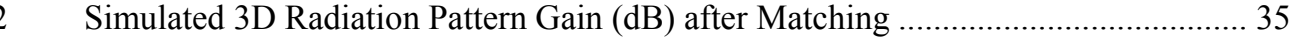 \\
\hline 3.5.3 & Simulated 2D Radiation Pattern............. \\
\hline 3.5.4 & Simulated S11 Reflection Coefficient (dB).. \\
\hline 3.5 .5 & Simulated Voltage Standing Wave Ratio (VSWR) ................... \\
\hline 3.6 & Compact Meandering-Monopole Simulation... \\
\hline 3.6.1 & Antenna Orientation and Matching Circuit.. \\
\hline 3.7 & Simulated 3D Radiation Pattern Gain (dB) after Matching. \\
\hline 3.7 .1 & Simulated 2D Radiation Pattern after Matching ..... \\
\hline 3.7 .2 & 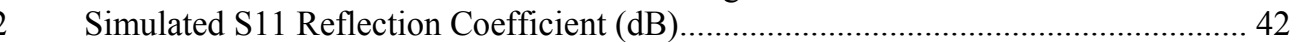 \\
\hline 3.7 .3 & Simulated Voltage Standing Wave Ratio (VSWR).. \\
\hline & 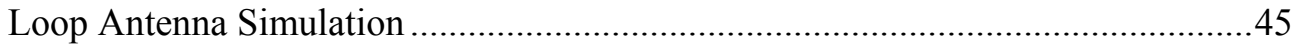 \\
\hline 3.8.1 & Antenna Orientation and Matching Circuit ...................... \\
\hline
\end{tabular}


3.8.2 Simulated 3D Radiation Pattern Gain (dB) after Matching ...................................... 45

3.8.3 Simulated 2D Radiation Pattern after Matching ......................................................... 46

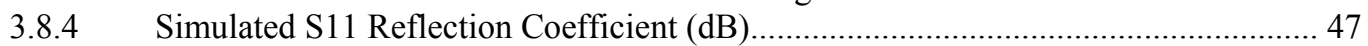

3.8.5 Simulated Voltage Standing Wave Ratio (VSWR) .................................................... 49

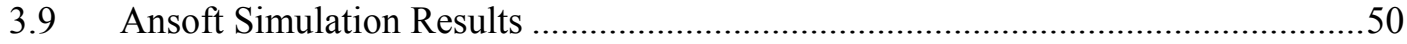

$4 \quad$ Prototype and Test Capabilities.......................................................................... 52

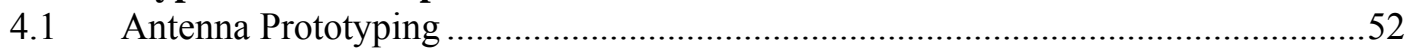

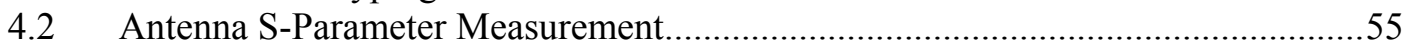

4.3 Antenna Radiation Pattern Measurement ................................................................56

5 Prototype-Antenna Measurement Results ........................................................................ 58

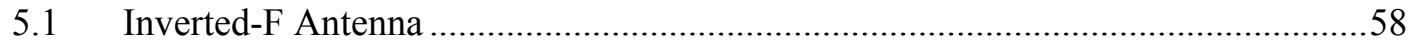

5.1.1 Antenna Orientation and Matching Circuit .............................................................. 58

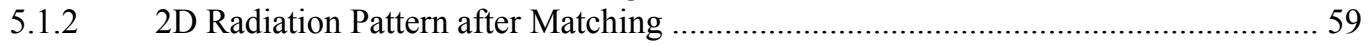

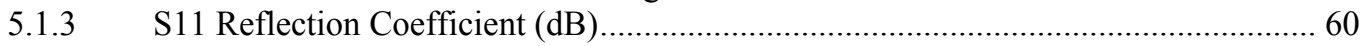

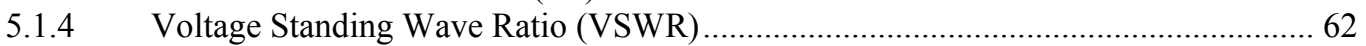

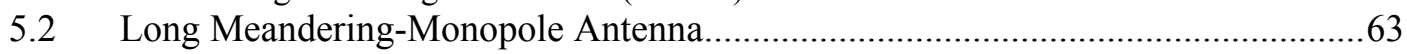

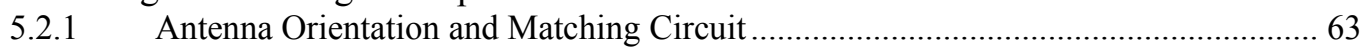

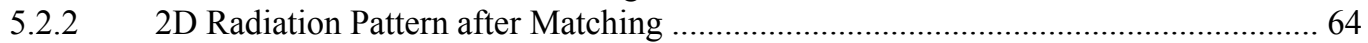

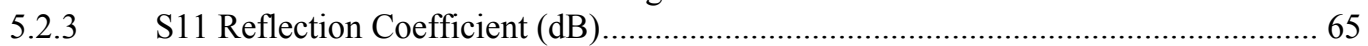

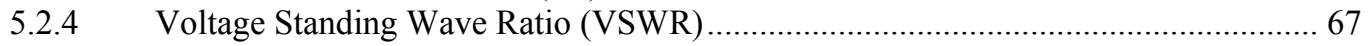

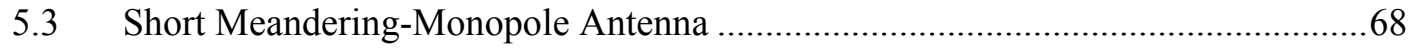

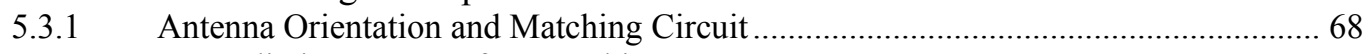

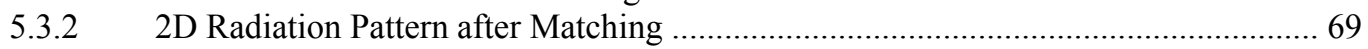

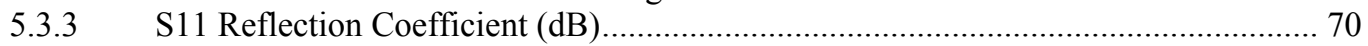

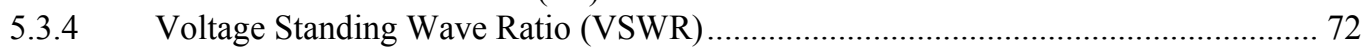

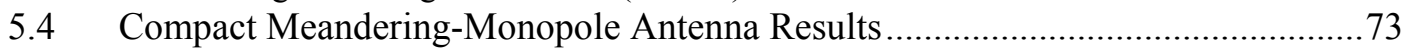

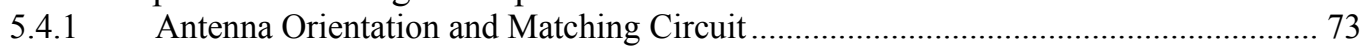

5.4.2 2D Radiation Pattern after Matching ..................................................................... 74

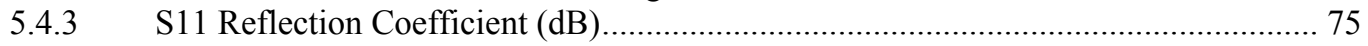

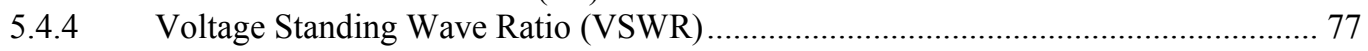

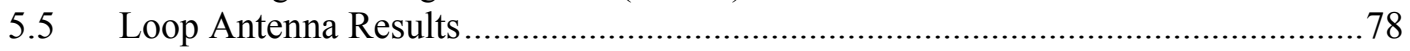

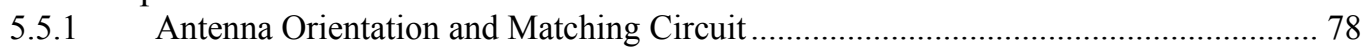

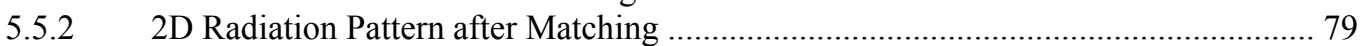

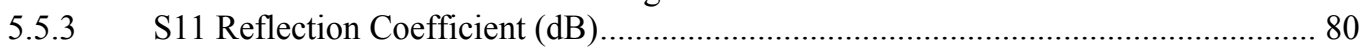

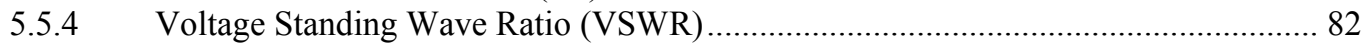

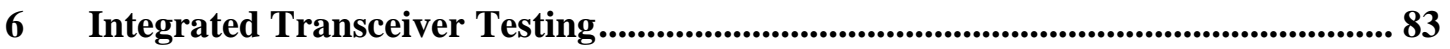

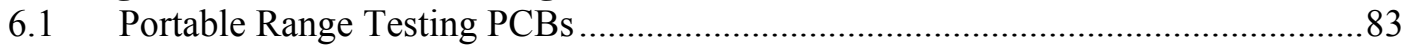

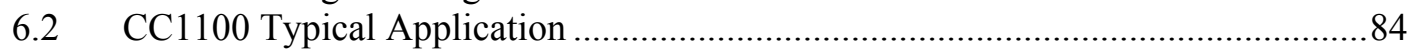

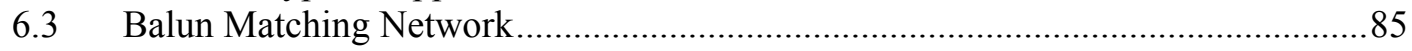

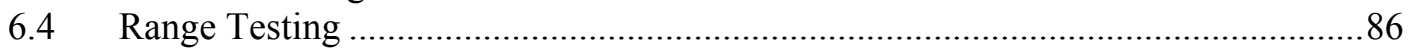

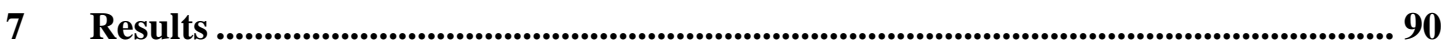

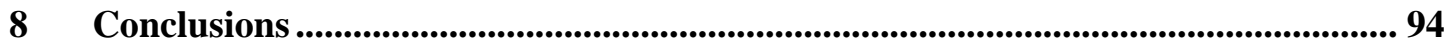

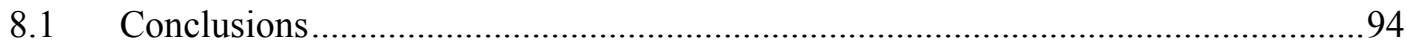

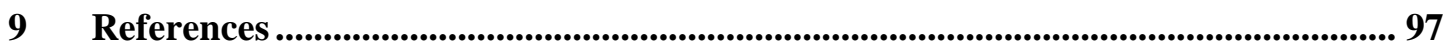




\section{List of Figures:}

Figure 2.1.1: Antenna with keepout under PCB and removal of ground plane. [2] .............................. 9

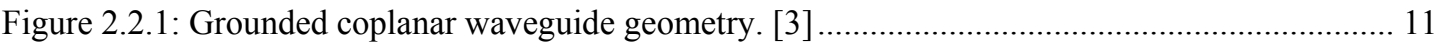

Figure 2.2.2: Grounded coplanar waveguide dimensions for 50-Ohm antenna feed............................ 11

Figure 2.7.1: Structure and nomenclature of right-angled microstrip bend. [6] .................................... 16

Figure 2.7.2: Geometry of the CPW-fed inverted-F monopole antenna................................................. 17

Figure 2.7.3: Geometry of the CPW-fed long meandering-monopole antenna...................................... 17

Figure 2.7.4: Geometry of the CPW-fed short meandering-monopole antenna. ..................................... 18

Figure 2.7.5: Geometry of the CPW-fed compact meandering-monopole antenna.............................. 19

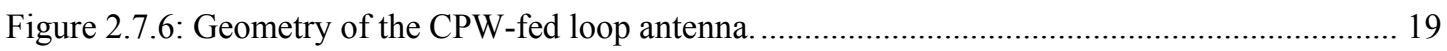

Figure 3.1.1: Ansoft HFSS simulation models (top view) for all antennas. From top-to-bottom: (a) Inverted-F antenna, (b) Long meandering-monopole antenna, (c) Short meandering-monopole antenna, (d) Compact meandering-monopole antenna, and (e) Loop antenna.

Figure 3.2.1: Ansoft HFSS simulation models (isometric view) for all antennas. From top-tobottom: (a) Inverted-F antenna, (b) Long meandering-monopole antenna, (c) Short meandering-monopole antenna, (d) Compact meandering-monopole antenna, and (e) Loop antenna.

Figure 3.3.1: Inverted-F antenna isometric orientation and simulated matching network required for 50-Ohm match.

Figure 3.3.2: Inverted-F antenna simulated 3D-radiation pattern expressed as gain in $\mathrm{dB}$ for the isometric orientation before addition of a matching network.

Figure 3.3.3: Inverted-F antenna simulated 2D-radiation pattern expressed as gain in $\mathrm{dB}$ for $\mathrm{XY}$, $\mathrm{XZ}$, and $\mathrm{YZ}$ planes before addition of a matching network

Figure 3.3.4: Inverted-F antenna input-port reflection coefficient (S11) expressed in dB before addition of a matching network

Figure 3.3.5: Inverted-F antenna input-port reflection coefficient (S11) expressed in $\mathrm{dB}$ after addition of a matching network.

Figure 3.3.6: Inverted-F antenna input-port reflection coefficient (smith chart) before addition of a matching network

Figure 3.3.7: Inverted-F antenna input-port reflection coefficient (smith chart) after addition of a matching network.

Figure 3.3.8: Inverted-F antenna input-port Voltage Standing Wave Ratio (VSWR) before addition of a matching network.

Figure 3.3.9: Inverted-F antenna input-port Voltage Standing Wave Ratio (VSWR) after addition of a matching network.

Figure 3.4.1: Long meandering-monopole isometric orientation and simulated matching network required for $50-\mathrm{Ohm}$ match.

Figure 3.4.2: Long meandering-monopole simulated 3D-radiation pattern expressed as gain in $\mathrm{dB}$ for the isometric orientation before addition of a matching network. 
Figure 3.4.3: Long meandering-monopole simulated 2D-radiation pattern expressed as gain in $\mathrm{dB}$ for XY, XZ, and YZ planes before addition of a matching network.

Figure 3.4.4: Long meandering-monopole input-port reflection coefficient (S11) expressed in $\mathrm{dB}$ before addition of a matching network

Figure 3.4.5: Long meandering-monopole input-port reflection coefficient (S11) expressed in $\mathrm{dB}$ after addition of a matching network.

Figure 3.4.6: Long meandering-monopole input-port reflection coefficient (smith chart) before addition of a matching network.

Figure 3.4.7: Long meandering-monopole input-port reflection coefficient (Smith Chart) after addition of a matching network.

Figure 3.4.8: Long meandering-monopole input-port Voltage Standing Wave Ratio (VSWR) before addition of a matching network.

Figure 3.4.9: Long meandering-monopole input-port Voltage Standing Wave Ratio (VSWR) after addition of a matching network.

Figure 3.5.1: Short meandering-monopole isometric orientation and simulated matching network required for $50-\mathrm{Ohm}$ match.

Figure 3.5.2: Short meandering-monopole simulated 3D-radiation pattern expressed as gain in $\mathrm{dB}$ for the isometric orientation before addition of a matching network.

Figure 3.5.3: Short meandering-monopole simulated 2D-radiation pattern expressed as gain in $\mathrm{dB}$ for XY, XZ, and YZ planes before addition of a matching network.

Figure 3.5.4: Short meandering-monopole input-port reflection coefficient (S11) expressed in dB before addition of a matching network.

Figure 3.5.5: Short meandering-monopole input-port reflection coefficient (S11) expressed in dB after addition of a matching network.

Figure 3.5.6: Short meandering-monopole input-port reflection coefficient (Smith Chart) before addition of a matching network.

Figure 3.5.7: Short meandering-monopole input-port reflection coefficient (Smith Chart) after addition of a matching network.

Figure 3.5.8: Short meandering-monopole input-port Voltage Standing Wave Ratio (VSWR) before addition of a matching network.

Figure 3.5.9: Short meandering-monopole input-port Voltage Standing Wave Ratio (VSWR) after addition of a matching network.

Figure 3.6.1: Compact meandering-monopole isometric orientation and simulated matching network required for 50-Ohm match.

Figure 3.7.1: Compact meandering-monopole simulated 3D-radiation pattern expressed as Gain in $\mathrm{dB}$ for the isometric orientation before addition of a matching network.

Figure 3.7.2: Compact meandering-monopole simulated 2D-radiation pattern expressed as Gain in $\mathrm{dB}$ for $\mathrm{XY}, \mathrm{XZ}$, and $\mathrm{YZ}$ planes before addition of a matching network.

Figure 3.7.3: Compact meandering-monopole input-port reflection coefficient (S11) expressed in $\mathrm{dB}$ before addition of a matching network.

Figure 3.7.4: Compact meandering-monopole input-port reflection coefficient (S11) expressed in $\mathrm{dB}$ after addition of a matching network. 
Figure 3.7.5: Compact meandering-monopole input-port reflection coefficient (Smith Chart) before addition of a matching network.

Figure 3.7.6: Compact meandering-monopole input-port reflection coefficient (Smith Chart) after addition of a matching network.

Figure 3.7.7: Compact meandering-monopole input-port Voltage Standing Wave Ratio (VSWR) before addition of a matching network.

Figure 3.7.8: Compact meandering-monopole input-port Voltage Standing Wave Ratio (VSWR) after addition of a matching network

Figure 3.8.1: Loop antenna isometric orientation and simulated matching network required for 50Ohm match.

Figure 3.8.2: Loop antenna simulated 3D-radiation pattern expressed as gain in $\mathrm{dB}$ for the isometric orientation before addition of a matching network.

Figure 3.8.3: Loop antenna simulated 2D-radiation pattern expressed as gain in $\mathrm{dB}$ for $\mathrm{XY}, \mathrm{XZ}$, and YZ planes before addition of a matching network.

Figure 3.8.4: Loop antenna input-port reflection coefficient (S11) expressed in dB before addition of a matching network.

Figure 3.8.5: Loop antenna input-port reflection coefficient (S11) expressed in dB after addition of a matching network.

Figure 3.8.6: Loop antenna input-port reflection coefficient (Smith Chart) before addition of a matching network.

Figure 3.8.7: Loop antenna input-port reflection coefficient (Smith Chart) after addition of a matching network.

Figure 3.8.8: Loop antenna input-port Voltage Standing Wave Ratio (VSWR) before addition of a matching network.

Figure 3.8.9: Loop antenna input-port Voltage Standing Wave Ratio (VSWR) after addition of a matching network.

Figure 4.1.1: Microstation Gerber file, from top-to-bottom: (a) Inverted-F antenna, (b) Long meandering-monopole antenna, (c) Short meandering-monopole antenna, (d) Compact meandering-monopole antenna, and (e) Loop antenna.

Figure 4.1.2: LPKF Minicontac-RS through-hole electroplating bath

Figure 4.1.3: The LPKF automated protomat router and fabricated prototypes. 54

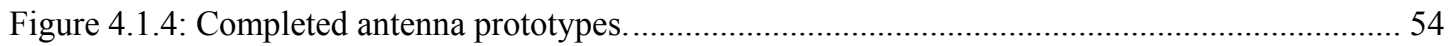

Figure 4.2.1: Agilent HP N3383A 300KHz-9GHz network analyzer.................................................. 55

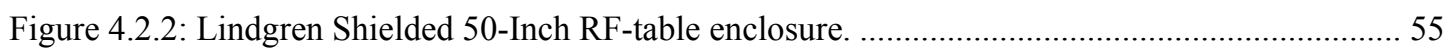

Figure 4.3.1: Equipment needed for radiation pattern measurement................................................ 56

Figure 5.1.1: Inverted-F antenna orientation and 50-Ohm matching network. .................................. 58

Figure 5.1.2: Inverted-F antenna 2D-radiation patterns expressed as gain in $\mathrm{dB}$ for $\mathrm{XY}, \mathrm{XZ}$, and $\mathrm{YZ}$ planes for horizontal and vertical polarization after addition of a matching network. 59

Figure 5.1.3: Inverted-F antenna input-port reflection coefficient (S11) expressed in dB before addition of a matching network. 
Figure 5.1.4: Inverted-F antenna input-port reflection coefficient (S11) expressed in dB after addition of a matching network.

Figure 5.1.5: Inverted-F antenna input-port reflection coefficient (Smith Chart) before addition of a matching network.

Figure 5.1.6: Inverted-F antenna input-port reflection coefficient (Smith Chart) after addition of a matching network.

Figure 5.1.7: Inverted-F antenna input-port Voltage Standing Wave Ratio (VSWR) before addition of a matching network.

Figure 5.1.8: Inverted-F antenna input-port Voltage Standing Wave Ratio (VSWR) after addition of a matching network.

Figure 5.2.1: Long meandering-monopole orientation and 50-Ohm matching network

Figure 5.2.2: Long meandering-monopole 2D-radiation pattern expressed as gain in $\mathrm{dB}$ for $\mathrm{XY}$, $\mathrm{XZ}$, and YZ planes after addition of a matching network.

Figure 5.2.3: Long meandering-monopole input-port reflection coefficient (S11) expressed in dB before addition of a matching network.

Figure 5.2.4: Long meandering-monopole input-port reflection coefficient (S11) expressed in $\mathrm{dB}$ after addition of a matching network.

Figure 5.2.5: Long meandering-monopole input-port reflection coefficient (Smith Chart) before addition of a matching network.

Figure 5.2.6: Long meandering-monopole input-port reflection coefficient (Smith Chart) after addition of a matching network.

Figure 5.2.7: Long meandering-monopole input-port Voltage Standing Wave Ratio (VSWR) before addition of a matching network.

Figure 5.2.8: Long meandering-monopole input-port Voltage Standing Wave Ratio (VSWR) after addition of a matching network.

Figure 5.3.1: Short meandering-monopole orientation and 50-Ohm matching network

Figure 5.3.2: Short meandering-monopole 2D-radiation pattern expressed as gain in $\mathrm{dB}$ for $\mathrm{XY}$, $\mathrm{XZ}$, and $\mathrm{YZ}$ planes after addition of a matching network.

Figure 5.3.3: Short meandering-monopole input-port reflection coefficient (S11) expressed in dB before addition of a matching network.

Figure 5.3.4: Short meandering-monopole input-port reflection coefficient (S11) expressed in dB after addition of a matching network.

Figure 5.3.5: Short meandering-monopole input-port reflection coefficient (Smith Chart) before addition of a matching network.

Figure 5.3.6: Short meandering-monopole input-port reflection coefficient (Smith Chart) after addition of a matching network.

Figure 5.3.7: Short meandering-monopole input-port Voltage Standing Wave Ratio (VSWR) before addition of a matching network.

Figure 5.3.8: Short meandering-monopole Input-Port Voltage Standing Wave Ratio (VSWR) after

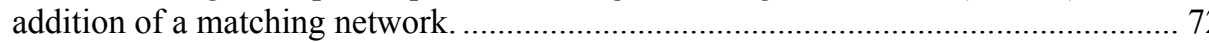

Figure 5.4.1: Compact meandering-monopole orientation and 50-Ohm matching network. 
Figure 5.4.2: Compact meandering-monopole 2D-radiation pattern expressed as gain in $\mathrm{dB}$ for $\mathrm{XY}$, $\mathrm{XZ}$, and $\mathrm{YZ}$ planes after addition of a matching network.

Figure 5.4.3: Compact meandering-monopole input-port reflection coefficient (S11) expressed in $\mathrm{dB}$ before addition of a matching network.

Figure 5.4.4: Compact meandering-monopole input-port reflection coefficient (S11) expressed in $\mathrm{dB}$ after addition of a matching network.

Figure 5.4.5: Compact meandering-monopole input-port reflection coefficient (Smith Chart) before addition of a matching network.

Figure 5.4.6: Compact meandering-monopole input-port reflection coefficient (Smith Chart) after addition of a matching network.

Figure 5.4.7: Compact meandering-monopole input-port Voltage Standing Wave Ratio (VSWR) before addition of a matching network.

Figure 5.4.8: Compact meandering-monopole input-port Voltage Standing Wave Ratio (VSWR) after addition of a matching network.

Figure 5.5.1: Compact meandering-monopole orientation and 50-Ohm matching network.

Figure 5.5.2: Loop antenna 2D-radiation pattern expressed as gain in $\mathrm{dB}$ for $\mathrm{XY}, \mathrm{XZ}$, and $\mathrm{YZ}$ planes after addition of a matching network......

Figure 5.5.3: Loop antenna input-port reflection coefficient (S11) expressed in dB before addition of a matching network.

Figure 5.5.4: Loop antenna input-port reflection coefficient (S11) expressed in dB after addition of a matching network.

Figure 5.5.5: Loop antenna input-port reflection coefficient (Smith Chart) before addition of a matching network.

Figure 5.5.6: Loop antenna input-port reflection coefficient (Smith Chart) after addition of a matching network.

Figure 5.5.7: Loop antenna input-port Voltage Standing Wave Ratio (VSWR) before addition of a

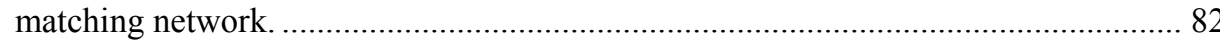

Figure 5.5.8: Loop antenna input-port Voltage Standing Wave Ratio (VSWR) after addition of a

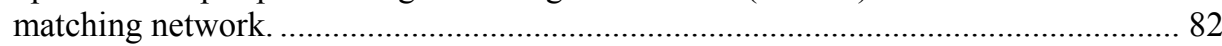

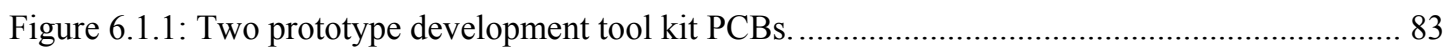

Figure 6.2.1: Reference circuit schematic provided by Chipcon (9) .................................................. 84

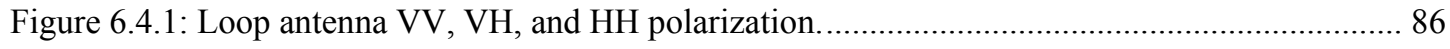

Figure 6.4.2: Face-to-Face, Side-to-Side, and Back-to-Back orientations in VV polarization................ 87

Figure 6.4.3: Reference monopole and HP Agilent 20-dB adjustable attenuator.................................... 87

Figure 6.4.4: Plot of signal strength vs range for differing loop antenna orientation in VV

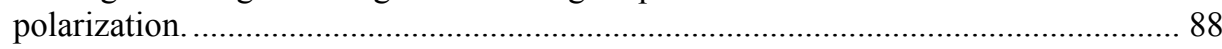

Figure 6.4.5: Plot of transmitted signal strength vs range for loop antenna polarizations...................... 89 


\begin{abstract}
This work focuses on the design and evaluation of the inverted-F, meandering-monopole, and loop antenna geometries. These printed antennas are studied with the goal of identifying which is suitable for use in a miniaturized transceiver design and which has the ability to provide superior performance using minimal Printed Circuit Board (PCB) space. As a result, the main objective is to characterize tradeoffs and identify which antenna provides the best compromise among volume, bandwidth and efficiency. For experimentation purposes, three types of meandering-monopole antenna are examined resulting in five total antennas for the study.
\end{abstract}

The performance of each antenna under study is evaluated based upon return loss, operational bandwidth, and radiation pattern characteristics. For our purposes, return loss is measured using the S11-port reflection coefficient which helps to characterize how well the small antenna is able to be efficiently fed. Operational bandwidth is measured as the frequency range over which the antenna maintains 2:1 Voltage Standing Wave Ratio (VSWR) or equivalently has 10-dB return loss. Ansoft High Frequency Structure Simulator (HFSS) is used to simulate expected resonant frequency, bandwidth, VSWR, and radiation pattern characteristics. Ansoft HFSS simulation is used to provide a good starting point for antenna design before actual prototype are built using an LPKF automated router. Simulated results are compared with actual measurements to highlight any differences and help demonstrate the effects of antenna miniaturization. Radiation characteristics are measured illustrating how each antenna is affected by the influence of a non-ideal ground plane. The antenna with outstanding performance is further evaluated to determine its maximum range of communication. Each designs range performance is evaluated using a pair of transceivers to demonstrate round-trip communication. 
This research is intended to provide a knowledge base which will help decrease the number of design iterations needed for future implementation of products requiring integration of small printed antennas. In the past, several design iterations have been needed to fine tune antenna dimensions and achieve acceptable levels of performance. This process consumes a large amount of time and material resources leading to costly development of transceiver designs. Typically, this occurs because matching components and antenna geometries are almost never correct on the first design. This work hopes to determine the limitations associated with antenna miniaturization and provide well known antenna examples that can be easily used in future work. 


\section{Introduction}

\subsection{Motivation}

Printed antennas have been an area of interest for many years, are fairly well known, and are widely used for a large variety of applications. It is understood that antenna length should be half a wavelength or larger to efficiently transform a guided wave into radiated energy. As technology gets smaller, many applications do not have room to properly implement the required antenna. In these cases, designers are commonly forced to use electrically small antennas which require careful tuning to achieve efficient operation. Electrically small refers to any antenna with its largest dimension measuring less than one-tenth of a wavelength at the desired operating frequency. These antennas when used improperly, may still work, but do not achieve the optimized performance. A tradeoff exists to design the smallest possible antenna while satisfying requirements for bandwidth, efficiency, and radiation characteristics.

If designed properly, these antennas can offer exceptional performance in very little space. According to Bancroft [1], printed microstrip antenna designs are ideal because of their main advantages: "low-cost fabrication, easily conformable to curved surfaces, resistant to shock and vibration, designs readily produce linear or circular polarization, considerable range of available gain and pattern options ( 2.5 to 10.0 $\mathrm{dBi}$ ), other devices realizable in microstrip may be integrated into microstrip antennas with no extra fabrication steps (e.g., branchline hybrid to produce circular polarization or incorporate a feed network for an array of microstrip antennas), and the antenna thickness (profile) is small.” In addition, they can be easily mass produced, do not require cavity backing, have low scattering cross section, and their matching may be integrated into the antenna structure. 
Great performance comes at a price, as designs must take into account nontrivial influences on antenna impedance caused by layout and packaging. To perform satisfactorily, according to Chen [2], "behavior of small unbalanced antennas is strongly dependent on the geometry of its ground plane. There is a direct correlation between the available impedance bandwidth and the length of the antennas ground plane." Small antennas are greatly affected by their immediate surroundings and in most cases the surrounding ground plane has more of an effect on the radiation pattern than the antenna itself. In extreme situations, the antenna element is merely used to adjust the desired frequency of operation. In addition, an asymmetrical ground plane causes additional gain in the direction of the extended ground plane and higher peak gain. A more symmetrical ground plane causes an omni directional radiation pattern with lower peak gain. It has been historically shown that unintentional objects will adversely affect the performance of small antennas when least expected. According to Bancroft [1], "there are several disadvantages such as narrow bandwidth (5\% to $10 \%$ [2:1 VSWR] is typical without special techniques), dielectric losses can be large for thin patches resulting in poor antenna efficiency, and sensitivity to environmental factors such as temperature and humidity." In these designs, it is important to build antennas that function extremely well.

The design phase holds several critical tradeoffs ultimately determining the maximum attainable performance of the antenna. This type of work must be done correctly the first time; once a design has been finalized, it is faithfully reproduced without any further modifications. Due to the copy-and-paste nature of society, marginal transceiver designs have a way of living forever. It is important to develop systems that function to the best of their ability with the very best matching possible each time as there is a good chance designs will be reused repeatedly without any additional validation of correctness. This importance is a significant motivational factor behind this paper's objective and why this work is being performed. 
The first objective of this work is to compare advantages and disadvantages of each antenna. Each antenna type has differing characteristics making it ideal for specific situations. To evaluate performance in a manner suitable for comparison, each antenna is identically implemented using a 50-Ohm Grounded Coplanar Waveguide (CPW) as its feeding line. The GCPW will allow each antenna to be independently assessed using a Vector Network Analyzer (VNA) for measurement of input-port reflection coefficient, efficiency, and bandwidth. Specialized turntable equipment will be used to measure antenna radiation patterns and characterize the antennas sensitivity to horizontally and vertically polarized data. The benefits and drawbacks of each design will be summarized in the results section and it will be shown that no single type of antenna is ideal for every situation.

The second objective of this work is to investigate the performance of these antennas in real world conditions. For that reason, the best performing antenna will be subjected to additional range testing to determine the maximum attainable range in a free-space environment. A custom set of range testing PCBs containing a CC1100 transceiver, balun circuit, and 50-Ohm matching network is used to perform antenna evaluation. Two range testing PCBs are used to determine signal strength with respect to distance for three differing polarizations of the antenna under test. Maximum path loss and attenuation can theoretically be calculated using Friis transmission formula, however, due to background noise and varying transceiver sensitivity levels, link budget calculations differ from real world conditions. It will be shown that range testing measurements can be used to estimate a realistic link budget for the system being evaluated. 


\subsection{Overview}

The challenge of this project is to design a miniaturized printed antenna for use in an integrated transceiver design capable of reliable communication over 200 feet. The entire design, including all radio, balun, and antenna circuitry must fit on a single sided FR4 PCB with dimensions of 0.030 " thick, 0.440 " high, and 1.6 " wide. The design must accommodate the printed antennas very low input impedance yet must still be reliable and easy to manufacture. All circuitry components including the transceiver chip, reference crystal, and matching network must fit into the predefined ground plane area. A 0.200 " epoxy layer will eventually be used to ruggedize the design and the antenna matching circuitry must have the ability to accommodate additional detuning caused by the packaging process.

For compatibility with legacy products, the frequency of operation is centered at $900 \mathrm{MHz}$ using Gaussian Frequency Shift Keying (GFSK) and frequency deviation of $1.5 \mathrm{kHz}$. The data baud rate is defined to be 38400 bits-per-second. The transceiver must be capable of offering Link Quality (LQ) and Received Signal Strength Indication (RSSI) for incoming messages. The input power is limited to 3.3 volts with $100 \mathrm{~mA}$ of current draw. External power amplifiers may be used as long as current consumption remains within budget. Low powered sleep is desired to accommodate battery powered operation. Plans are to leverage previous knowledge and utilize microprocessor firmware already written to control the Chipcon CC1101 transceiver using 3-wire SPI serial bus (SCLK, SO, and SI). The transceiver register map, radio state machine, and command listing are compatible with the entire CC11xx series of devices. 


\subsection{Applications}

The information contained in this document may pertain, but is not limited to the following applications: Satellite Communication, RADAR, Telemetry, Environmental Instrumentation, Remote Sensing, Wireless Sensors, Large Antenna Feed Elements, Navigation Receivers, Key Fobs, Medical Implants, ect. This is not a complete exhaustive list but it shows the importance and wide extent of applications where printed antennas are frequently used. According to Bancroft [1], "a large number of commercial needs are met by the use of microstrip and printed antennas, these include the ubiquitous Global Positioning System (GPS), Zigbee, Bluetooth, WiMax, WiFi Applications, 802.11a,b,g and others." This list is almost certainly expected to grow as new technologies and commercial needs begin to emerge in the future.

\subsection{Definitions}

3-dB Beamwidth is the width of the antenna main-lobe measured between half-power points in degrees $3-\mathrm{dB}$ down from its maximum gain.

Antenna Radiation Pattern is a three dimensional graphical representation of the antennas radiation into space as a function of angular direction describing how the antenna radiates energy.

Antenna Resistance is the total real valued resistance of the antenna accounting for both radiation and ohmic-loss resistance.

Directivity is the ratio of maximum radiation intensity (power per unit solid angle) in a given direction to the intensity of an isotropic radiator averaged over a sphere is all directions. 
Efficiency is the ratio of radiated power to power available at the antennas input port. Antenna efficiency is directly related to antenna radiation resistance which is responsible for radiated power.

Gain is the ratio of the antennas radiated power in a given direction to the radiated power of an isotropic radiator. Gain is expressed in $\mathrm{dB}$ and is identical to directivity but it also includes the effects of antenna efficiency accounting for power in the antenna converted to heat.

Input Impedance is the complex impedance of the antenna at its terminals presented on a Smith chart with real and imaginary parts.

Isotropic Radiator is an imaginary antenna that has a gain of $0-\mathrm{dB}$ and radiates power equally in all directions and has a perfectly spherical radiation pattern.

Null is a direction where radiated power is nonexistent or minimal in comparison to main lobe radiated power.

Loss Resistance is part of the antenna's total resistance converted to heat and relates to the power dissipated within the antenna.

Radiation Resistance is part of the antenna's total resistance caused by radiated electromagnetic waves and relates to radiated power.

Sidelobe is any other lobe not considered to be the main lobe.

VSWR Bandwidth is defined as the frequency range over which the Antenna has a VWSR of 2:1. At a VSWR of $2: 1$, the S11-reflection coefficient is $-10 \mathrm{~dB}$, 
approximately 90 percent of the incident power is radiated and 10 percent of the incident power is reflected.

VSWR is the Voltage Standing Wave Ratio defined as the ratio of reflected voltage to incident voltage in a standing wave pattern developed when power is reflected from a load. The VSWR is used to characterize the impedance mismatch between a source and a load and is another way of expressing reflection coefficient and how closely the source and load impedance are matched. In this work, VSWR expresses how closely the antenna is matched to its $50-\mathrm{Ohm}$ input port. 


\subsection{Acronyms}

\begin{tabular}{|c|c|}
\hline ADS & Advanced Design System \\
\hline AUT & Antenna Under Test \\
\hline BALUN & Balanced-to-Unbalanced Conversion \\
\hline CAD & Computer Aided Design \\
\hline $\mathrm{CPW}$ & Coplanar Waveguide \\
\hline DAMS & Desktop Antenna Measurement Studio \\
\hline EM & Electromagnetic \\
\hline FR4 & Flame Retardant 4 \\
\hline GCPW & Grounded Coplanar Waveguide \\
\hline GFSK & Gaussian Frequency Shift Keying \\
\hline GPS & Global Positioning System \\
\hline HFSS & High Frequency Structure Simulator \\
\hline HPIB & Hewlett Packard Interface Bus \\
\hline LNA & Low Noise Amplifier \\
\hline LQ & Link Quality \\
\hline $\mathrm{LT}$ & Loss Tangent \\
\hline MDS & Minimum Detectable Signal \\
\hline PCB & Printed Circuit Board \\
\hline RSSI & Received Signal Strength Indication \\
\hline $\mathrm{SCK}$ & Serial Clock \\
\hline SI & Serial Input \\
\hline SMA & Sub-Miniature version A \\
\hline $\mathrm{SO}$ & Serial Output \\
\hline SPI & Serial Peripheral Interface \\
\hline TEM & Transverse Electromagnetic \\
\hline VNA & Vector Network Analyzer \\
\hline VWSR & Voltage Standing Wave Ratio \\
\hline
\end{tabular}




\section{Antenna Theory}

\subsection{Performance}

In order to optimize the amount of power radiated, the ground plane must be completely removed underneath the antenna. A keepout zone is added below the antenna where components are not to be placed. This concept is illustrated below in Figure 2.1.1. Removal of the ground plane ensures the printed antenna design does not follow conventional TEM microstrip design equations. However, the same principle that contributes to unwanted radiation loss in microstrip transmission lines is directly responsible for the radiation characteristics in planar antennas. Because of this similarity three brief sections are included to address microstrip width-to-depth ratio, characteristic impedance, and effective dielectric constant in sections $2.3-2.5$.

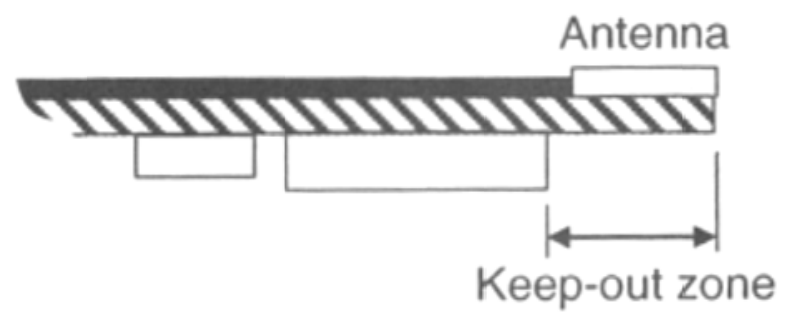

Figure 2.1.1: Antenna with keepout under PCB and removal of ground plane. [2]

An overall set of goals must be established for specific antenna performance before the design procedure can begin. A suitable dielectric substrate must be chosen, in this case, fiberglass reinforced $1 / 2$ oz copper-cladded FR4 epoxy with 0.028 " thickness. A list of antenna performance criterion has been established that is estimated will meet the project requirements of transmitting over 200 feet. If more than one of the evaluated antenna geometries outperforms specifications listed in sections 2.1.1and 2.1.2 the antenna with the largest ground plane having the most component placement room will be selected for additional range testing. 


\subsubsection{General Data}

Frequency $=900 \mathrm{MHz}$

Bandwidth $=20 \mathrm{kHz}$

Operating Temperature $=-30{ }^{\circ} \mathrm{C}$ to $+80{ }^{\circ} \mathrm{C}$

Radiation Characteristics $=$ Dependent on loop geometry.

Polarization $=$ Linear

Power handling capability $=10 \mathrm{~mW}$

Weight $=0.1 \mathrm{~g}$

Type $=$ Low height, SMD

Max Dimensions = 1.6" x 0.44" x 0.030"

Input impedance with matching $=50 \Omega$

\subsubsection{Electrical Specifications}

Peak Gain $=2.1 \mathrm{dBi}$

Average Gain $=-1.2 \mathrm{dBi}$

Average Efficiency (e) $=75 \%$ (estimated)

Maximum Return Loss $=-11 \mathrm{~dB}$

Maximum VSWR $=1.8: 1$

\subsection{Grounded Coplanar Waveguide Feed}

To avoid an impedance mismatch, each antenna feed is constructed with a Grounded Coplanar Waveguide (GCPW) transmission line. The equations needed to solve for a GCPW are still empirical and outside the scope of this text. However, electromagnetic field solving programs such as Advanced Design System (ADS) by Agilent can be used for simulation of GCPW geometry to find the proper trace width and ground plane separation size. The geometry of GCPW is shown Figure 2.2.1. 


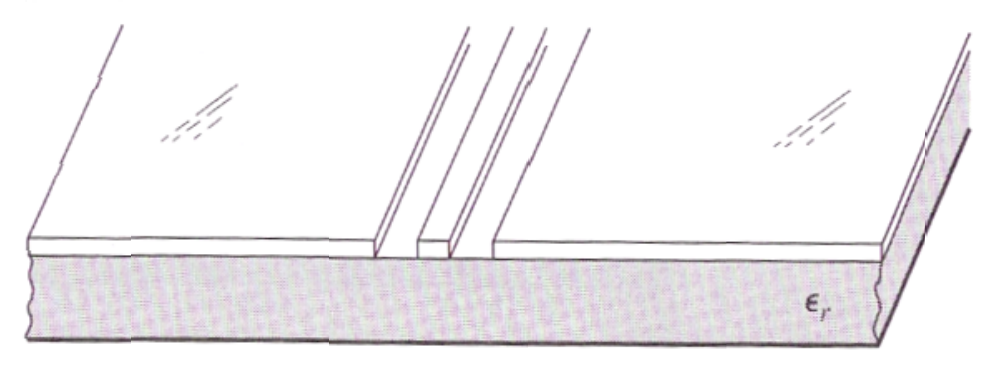

Figure 2.2.1: Grounded coplanar waveguide geometry. [3]

According to Waterhouse [4], "Coplanar Waveguides are increasingly used in microstrip circuits due to easy direct integration of active solid-state devices within the antenna. Coplanar Waveguides have low radiation loss, low dispersion, good control of characteristic impedance, and uniplanar configuration." Numerical modeling for GCPW assumes a conductor material deposited on substrate surrounded only by air. The following dimensions in Figure 2.2.2 were calculated using the ADS transmission line calculator. This configuration is able to achieve the needed $50-\mathrm{Ohm}$ characteristic impedance and key parameters are setup as follows: PCB dielectric constant $\varepsilon_{r}=4.4$, copper foil thickness $t=0.0007$ ", operating frequency $f_{0}=$ $900 \mathrm{MHz}$, substrate height $h=0.028 "$, ground plane separation $s=0.006 "$, and conductor width $w=0.030 "$

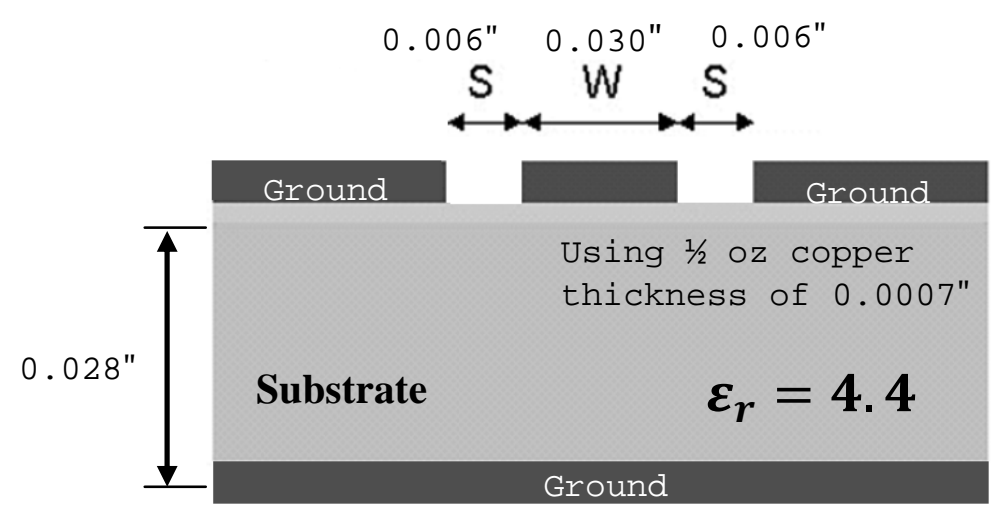

Figure 2.2.2: Grounded coplanar waveguide dimensions for 50-Ohm antenna feed. 


\subsection{Microstrip Width-to-Depth Ratio}

The following TEM equations are used to describe the behavior of microstrip transmission line suspended above a ground plane by dielectric material. According to Pozar [3], "the conductor width $d$, to substrate depth $W$, can be determined as the ratio $W / d$ given a characteristic impedance $Z_{0}$ and substrate dielectric constant $\epsilon_{r}$." The following two equations are used to calculate proper microstrip width-to-depth ratio:

$$
\frac{W}{d}=\left\{\begin{array}{cc}
\frac{8 e^{A}}{e^{2 A}-2} & (\text { for } W / d<2) \\
\frac{2}{\pi}\left[B-1-\ln (2 B-1)+\frac{\epsilon_{r}-1}{2 \epsilon_{r}}\left\{\ln (B-1)+0.39-\frac{0.61}{\epsilon_{r}}\right\}\right] & (\text { for } W / d>2)
\end{array}\right\}
$$

Where,

$$
\begin{aligned}
& A=\frac{Z_{0}}{60} \sqrt{\frac{\epsilon_{r}+1}{2}}+\frac{\epsilon_{r}-1}{\epsilon_{r}+1}\left(0.23+\frac{0.11}{\epsilon_{r}}\right) \\
& B=\frac{377 \pi}{2 Z_{0} \sqrt{\epsilon_{r}}}
\end{aligned}
$$

An initial assumption must be made for $W / d$ to be $>2$. If the calculation for $W / d$ then yields a number less than 2 , this calculation is repeated again using the equation for $W / d<2$.

Width-to-depth ratio is generally calculated with some preferred characteristic impedance in mind. For example, if the microstrip's characteristic impedance is desired to be 50-Ohms, given a characteristic impedance of $Z_{0}=50 \mathrm{ohms}$ and FR4 dielectric constant $\epsilon_{r}=4.4$, the resulting width-to-depth ratio is $W / d=1.91$. With a substrate depth $d=0.028 "$, the ideal calculated microstrip width is $W=0.053 "$. 


\subsection{Microstrip Characteristic Impedance}

According to Pozar [3], "the characteristic impedance can be calculated for a microstrip if conductor width and substrate depth are known."

$$
Z_{0}=\left\{\begin{array}{cl}
\frac{60}{\sqrt{\epsilon_{l}} \ln \left(\frac{8 d}{W}+\frac{W}{4 d}\right)} & (\text { for } W / d \leq 1) \\
\frac{120 \pi}{\sqrt{\epsilon_{l}}\left[\frac{W}{d}+1.393+0.667 \ln \left(\frac{W}{d}+1.444\right)\right]} & (\text { for } W / d \geq 1)
\end{array}\right\}
$$

For example, given a conductor width $W=0.020$ ", substrate depth $d=$ $0.028 "$, and effective dielectric constant $\varepsilon_{l}=3.16$, the resulting characteristic impedance of $Z_{0}=81.85 \mathrm{Ohms}$ can be calculated.

\subsection{Microstrip Effective Dielectric Constant}

The microstrip dielectric constant is altered because some of the electric fields surrounding its conductor exist in air while others exist in substrate. A new relative dielectric constant is calculated to account for wave propagation in the microstrip and represents an equivalent homogeneous medium accounting for effects of surrounding dielectric materials. According to Pozar [3], "the relative dielectric constant is calculated using the following equation if the conductor-width to substrate-depth ratio $W / d$ is $\geq 1: "$

$$
\varepsilon_{l}=\frac{\varepsilon_{r}+1}{2}+\frac{\varepsilon_{r}-1}{2} \frac{1}{\sqrt{1+12 d / W}}(\text { for } w / d \geq 1)
$$

However, according to Bahl and Trivedi [5], "if the width-to-depth ratio $W / d$ is $<1$ the following equation must be used:" 


$$
\varepsilon_{l}=\frac{\varepsilon_{r}+1}{2}+\frac{\varepsilon_{r}-1}{2}\left[\frac{1}{\sqrt{1+12 d / W}}+0.04\left(1-\left(\frac{W}{d}\right)^{2}\right)\right](\text { for } w / d<1)
$$

The effects of conductor thickness and frequency dispersion are ignored in each of the equations above. They are expressed only as a function of width-to-depth ratio and effective dielectric constant.

As an example, consider FR4 substrate with a dielectric constant $\varepsilon_{r}=4.4$, conductor width of 0.020 ", and substrate depth of $0.028 \%$. The resulting constant $\varepsilon_{l}=3.16$ is slightly less than the original. If the adjusted effective dielectric constant is greater than the original dielectric constant or less than $\epsilon_{r}=1$ for air, an error has occurred.

\subsection{Conductor Length}

To calculate the appropriate antenna conductor length, the desired free-space wavelength must be divided by the square root of the effective dielectric constant. In the following example, given a 900-MHz frequency and effective dielectric $\epsilon_{l}=$ 3.13, we find the desired $\lambda_{1 / 2}$ and $\lambda_{1 / 4}$ antenna lengths.

$$
\begin{aligned}
& \lambda_{1 / 2 \text { Freespace }}=\frac{c}{2 f}=16.67 \mathrm{~cm} \text { (6.56 inches) } \\
& \lambda_{1 / 2 \text { Effective }}=\frac{c}{2 f \sqrt{\epsilon_{l}}}=9.42 \mathrm{~cm} \text { (3.71 inches) } \\
& \lambda_{1 / 4 \text { Freespace }}=\frac{c}{4 f}=8.33 \mathrm{~cm} \text { (3.28 inches) } \\
& \lambda_{1 / 4 \text { Effective }}=\frac{c}{4 f \sqrt{\epsilon_{l}}}=4.71 \mathrm{~cm} \text { (1.85 inches) }
\end{aligned}
$$




\subsection{Geometries}

The following antenna structures are studied to determine which is ideally suited for use in an integrated transceiver. Each antenna's radiating-element layout is slightly different and is based only upon what length reasonably fits within each design. For this reason, each poses its own set of unique challenges when optimizing for maximized power output. To be flexible, a lumped four-element matching network is used. In this configuration, a Pi, T, or two-stage ladder network can be used if needed to accommodate the required 50-Ohm match.

Typically, the resonant frequency of the printed antenna is selected by adjusting its length. Because a matching network is being used, there is no physical requirement on antenna length. Actual radiating-element length in each design may be shorter or longer than optimal when taking into account the effects of dielectric constant and excited mode-of-resonance. A matching network is used to compensate for the effects of non-ideal length. If the antenna is being designed for specific impedance without a matching network in mind, it becomes very critical to precisely know the effective dielectric constant and mode of resonance to appropriately set the radiating-element's length. It becomes more difficult because any small variation in dielectric constant has a very large effect on optimal antenna length. Other challenges include finding a balance between antenna efficiency and operational bandwidth. There is more on this point in Section 2.8.

All antennas have chamfered corners to help compensate for capacitance, fringing effects, and impedance mismatch in right-angled microstrip bends. According to Edwards [6], "the optimal bend distance $b$ should be approximately 57 percent of the conductor width w", as illustrated in Figure 2.7.1. 


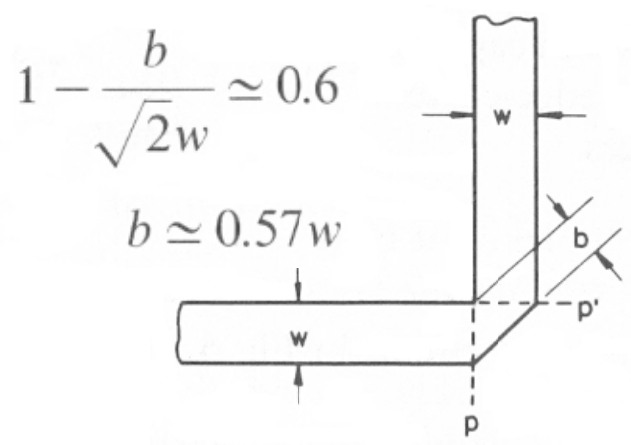

Figure 2.7.1: Structure and nomenclature of right-angled microstrip bend. [6]

Each antenna design uses a Grounded Coplanar Waveguide (GCPW) feed to provide 50-Ohm characteristic impedance. The GCPW measurements for trace width, ground plane separation, and substrate height have been calculated using the Agilent ADS line-calculator program. The antenna feedstrip for each design consists of a GCPW with a 0.030 " trace width and 0.006 " ground plane separation assuming a 0.0007 ' conductor thickness and dielectric constant of $\epsilon_{r}=4.4$ for FR4 substrate material.

With the exception of the inverted-F antenna, each antenna is driven in singleended unbalanced configuration and is matched for 50-Ohm input impedance. The inverted-F antenna is a balanced antenna and is tuned by simply adjusting radiating element length. To simplify test procedures, the connector for each antenna under test is standardized SMA end-feed jack.

The radiating element width and height is identical for each design, a 0.020 " trace width and a 0.0007 " conductor thickness has been established. As a general rule of thumb, the conductor width is denoted as (W) and a minimum separation of $1.5 *$ (W) is always maintained between the antenna and nearby objects, including ground plane and adjacent meandering-antenna segments. For each of the antennas, a 0.020 " radiating element width required at least a 0.030 " keepout zone be held between the antenna trace and neighboring structures. 
The first antenna, known as the inverted-F Antenna, is shown in Figure 2.7.2. According to Chen [7], to achieve good impedance matching, a shorting pin can be introduced close to the vertical segment so that a shunt-driven inverted-L antenna transmission with an open end - an inverted-F antenna - is formed." The following design is folded to increase the length of its thin-wire radiating element. Ansoft simulations are performed at $900 \mathrm{MHz}$ to find the optimal radiating-element length of 2.25 inches and shorting-pin-to-feed-line distance.

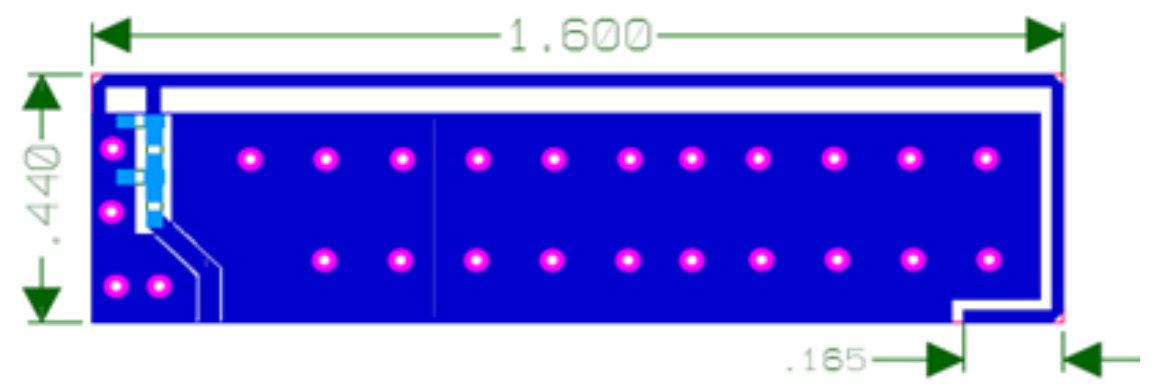

Figure 2.7.2: Geometry of the CPW-fed inverted-F monopole antenna.

The second antenna, known as the Long Meandering-Monopole Antenna, is shown in Figure 2.7.3. This antenna is modeled after a simple folded-monopole antenna and has the longest radiating-element length at 5.74 inches. The design of this antenna structure is known to be highly inefficient due to opposing currents that travel in opposite directions along parallel sections of antenna. The field-cancellation effect caused by these currents reduces the usefulness of this antenna. Even so, it has been included because it adds variety to the experiment and its long radiating element length can possibly make it easier to be matched.

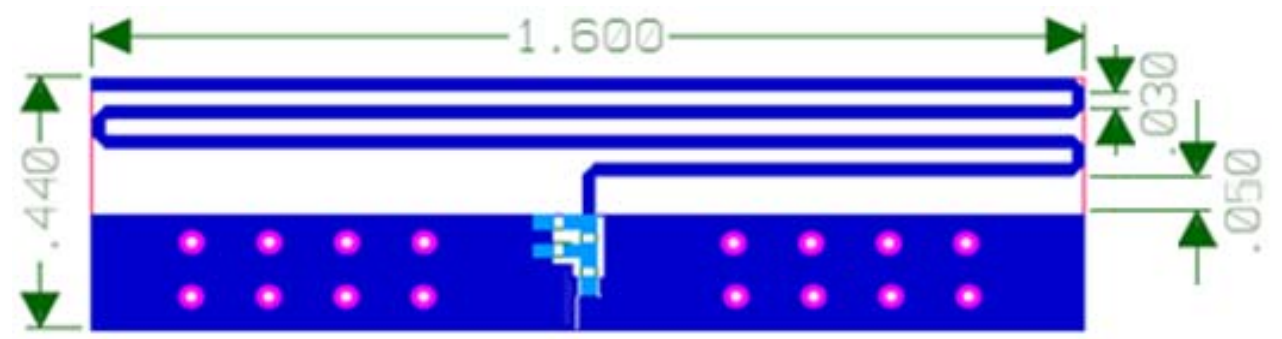

Figure 2.7.3: Geometry of the CPW-fed long meandering-monopole antenna. 
The third antenna, known as the short meandering-monopole antenna, is shown in Figure 2.7.4. This antenna is very similar to the long meandering monopole but its total length is only 4.11 inches. The Short Meandering Monopole is slightly longer than one half of a wavelength at $900 \mathrm{MHz}$. One meander has been removed to offer larger ground plane area providing additional room for component-placement. Similar to the long meandering monopole, this antenna suffers from inefficiencies due to opposing currents traveling in opposite directions along parallel sections of antenna. The field-cancellation effects caused by these currents will reduce the usefulness of this antenna. Even so, it has been included for comparison of its radiation pattern and ability to be matched.

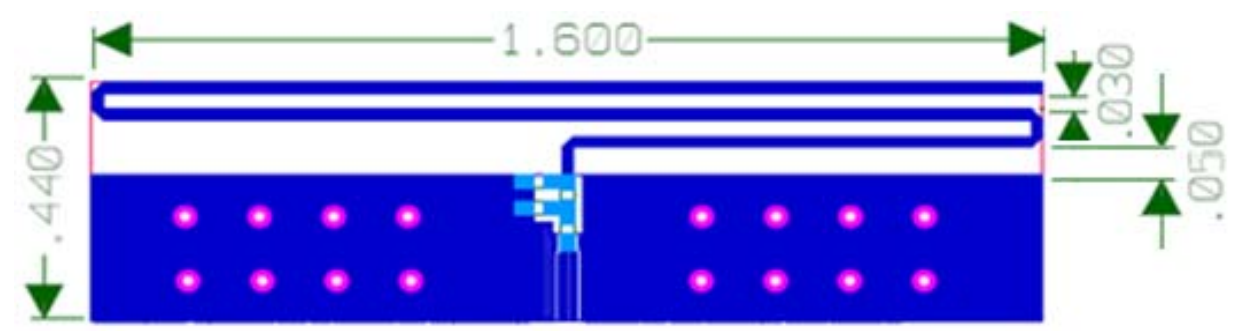

Figure 2.7.4: Geometry of the CPW-fed short meandering-monopole antenna.

The fourth antenna, known as the compact meandering-monopole antenna, is shown in Figure 2.7.5. This antenna has 10 meanders with a total length of 4.97 inches. It still based on a bent monopole but offers a different ground plane configuration than its previous two counterparts. This configuration is expected to have a slightly different radiation pattern because its orientation is rotated. The antenna efficiency will still suffer due to magnetic-field cancellation in neighboring conductors. However, radiation is still possible because this antenna is much longer and current cancellation effects will not occur in narrow "edge" portions of the antenna. 


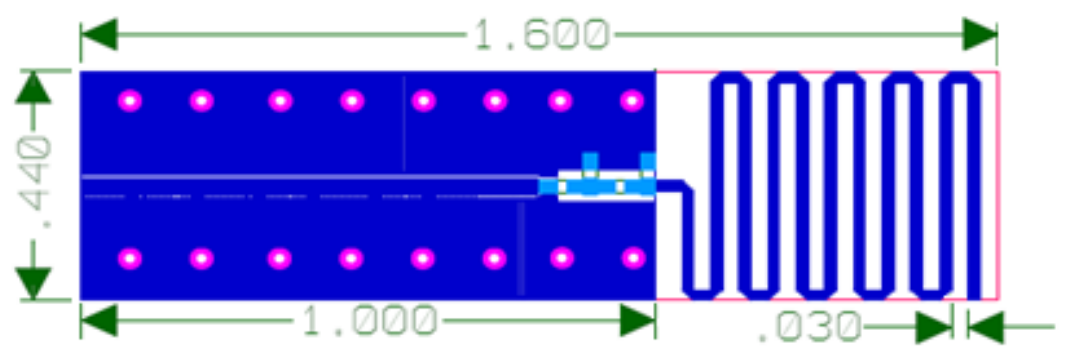

Figure 2.7.5: Geometry of the CPW-fed compact meandering-monopole antenna.

The fifth antenna, known as the loop antenna, is shown in Figure 2.7.6. This antenna has the distinct advantage of being configured as a bent monopole or a loop antenna depending on installation of a grounding inductor. For the purposes of this study, the end of the loop antenna is not shorted to the ground plane and is being used in the bent monopole configuration. The total 2.04-inch element length is slightly more than one-quarter wavelength at $900 \mathrm{MHz}$ when the effective dielectric constant is taken into account. The radiation pattern of this antenna is expected to resemble a monopole and be similar in appearance to the compact meandering monopole antenna mentioned above.

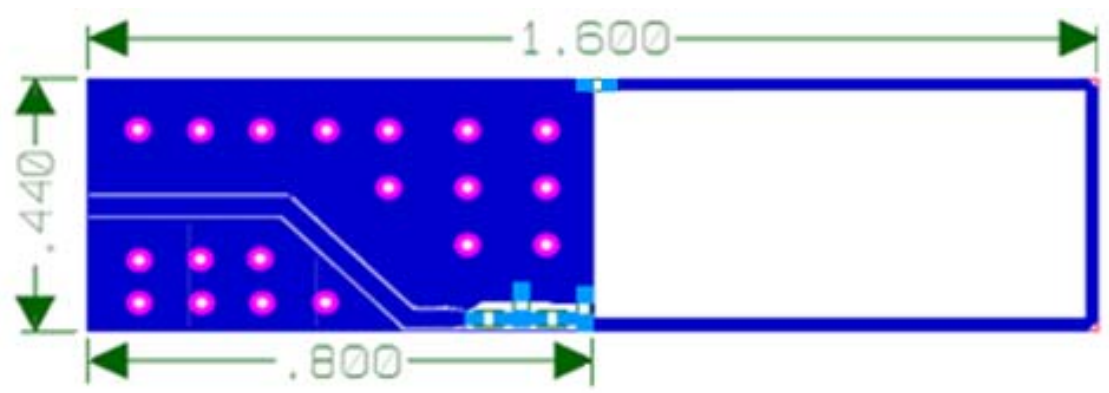

Figure 2.7.6: Geometry of the CPW-fed loop antenna. 


\subsection{Electrically Small Antennas}

According to Chen [2], "The operation of electrically small antennas is dictated by fundamental relationships which relate their minimum Q-factor to the volume of the smallest sphere in which they can be enclosed, often referred to as the Chu-Harrington limit." The Q-factor measures how much energy is stored verses energy that is radiated. Electrically small antennas are known for having reactive input impedances that must be countered by adding the opposite reactance. Typically, the reactance is capacitive and a small inductor is added in series with the antenna. Bear in mind, any additional lumped tuning elements will lower the quality factor reducing efficiency but has the ability to improve antenna bandwidth. The addition of lumped elements is a necessity for tuning the resonant frequency. However, caution must be used because additional elements will lower the Q-factor causing less power to be radiated by the antenna. We must choose the configuration which offers the highest possible efficiency while still maintaining the required operational bandwidth. By adding lumped elements the bandwidth is broadened but efficiency is sacrificed. This tradeoff must be carefully balanced and efficiency should be exchanged for bandwidth only when absolutely needed.

Antennas with a physically short length appear capacitive up to the frequency where the antennas are about one-quarter of a wavelength long. The radiation resistance is small and the capacitive reactance is large. Low radiation resistance means a large amount of current is required to produce any kind of significant radiation by the antenna. Tools that increase the antennas radiation resistance and reduce the amount of reactance are constantly being sought after. Because printed antennas have a smaller radiation resistance there is a significant portion of the power dissipated in matching circuitry that otherwise would have been radiated by the antenna. Put another way, the matching element loss due to resistance becomes large with respect to the antennas radiation resistance. 


\section{Ansoft Simulation}

Ansoft HFSS is state-of-the-art commercial design software for simulating Sparameter data and computing 3D full-wave electromagnetic (EM) fields. It is used

to simulate each antenna design to provide expected radiation pattern (gain), Voltage Standing Wave Ratio (VSWR), and S11 reflection coefficients. The challenge is to predict initial antenna performance and required matching circuitry needed to achieve 50-Ohms input impedance. Ansoft provides a great starting point for antenna design before moving forward to the prototyping phase. However, simulation will never be a replacement for prototyping because it is nearly impossible to model everything existing in real life.

The purpose of this exercise is to perform simulations predicting initial antenna behavior, implement the designs, and measure the actual results. If used correctly, HFSS can improve the probability of first-pass design success reducing the length of time needed for production ready designs. However, there are many reasons why simulated results might vary from real-world conditions. These reasons include the following: the fact that routed prototype edges are jagged not smooth, the antenna trace width varies with router-bit depth and is not constant, the copper thickness after plating is always thicker than $1 / 2 \mathrm{oz}$, and the SMA feeding structure has not been accounted for in the 3D model. The amount of difference caused by these variations is captured and can be incorporated into upcoming simulations to help achieve more precise results in the future.

Each model shown in Figure 3.1.1 and Figure 3.2.1 includes a fourcomponent matching network that is used to simulate the expected elements needed to achieve a 50-Ohm match. A driven terminal solution is setup to solve for data at the antenna input port. Initial results are simulated by replacing series elements with shorts (copper) and modeling shunt elements as open (air). A 50-Ohm lumped- 
element excitation port (shown in orange) is used to drive the matching networks input. The frequency sweep is setup for a $900-\mathrm{MHz}$ solution with 1601 points of data starting at $700 \mathrm{MHz}$ and ending at $1.1 \mathrm{GHz}$. The convergence is set to a Maximum Delta-S of 0.04 and the far-field radiation pattern is simulated using an $8 \times 8 \times 8$-inch air box. These parameters are important and small variation in size or aspect ratio will cause different simulation results to be attained.

The initial simulation is performed to capture the antenna's unaltered performance characteristics without any matching. The complex reflection coefficient for S11 is plotted on a Smith chart and used to calculate the appropriate matching network combination capable of adjusting initially simulated results to 50Ohms. This procedure is identical to the matching process performed in a laboratory for an actual antenna. The calculated matching network is then evaluated in the simulation by applying a Lumped-RLC condition to the appropriate 0402-size packages contained in the model. An iterative process is needed to fine tune the matching network, starting at the load impedance and moving toward the source, adding elements one by one, to ensure the calculated RLC value produces the expected Smith chart movement. Once refined, a final simulation is performed to capture expected performance characteristics of the final "matched" antenna. The simulated results are presented in the following Sections 3.3 - 3.8 in a "before matching" and "after matching" style format. This simulation method is being evaluated to determine if it can be an effective way to predict antenna performance. Simulated results from this simulation process are compared with actual measured results in Chapter 7 of the report. 


\subsection{Models (Top View)}

(a)

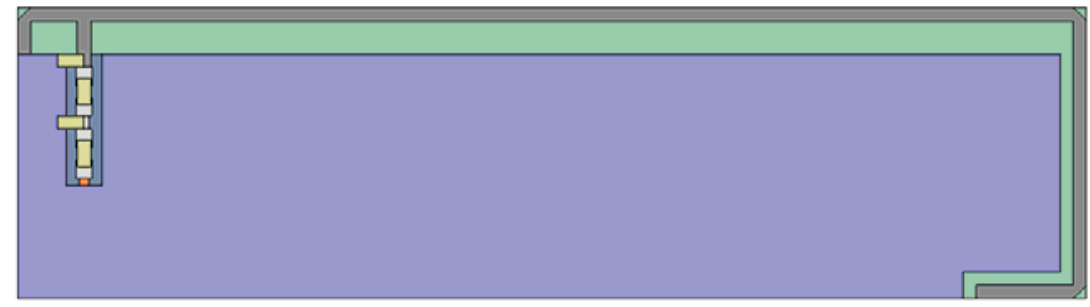

(b)

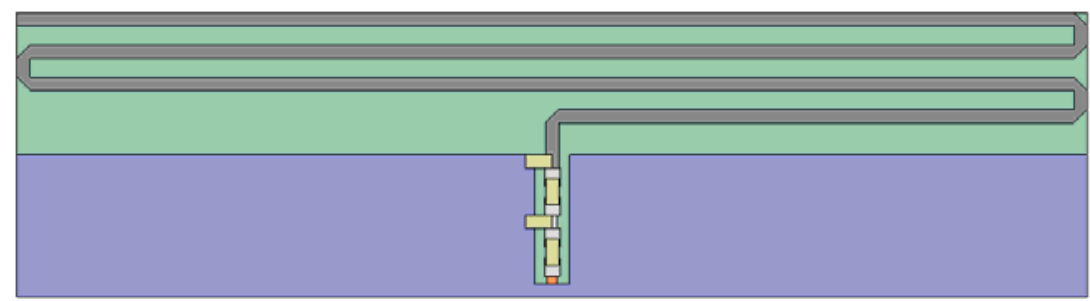

(c)

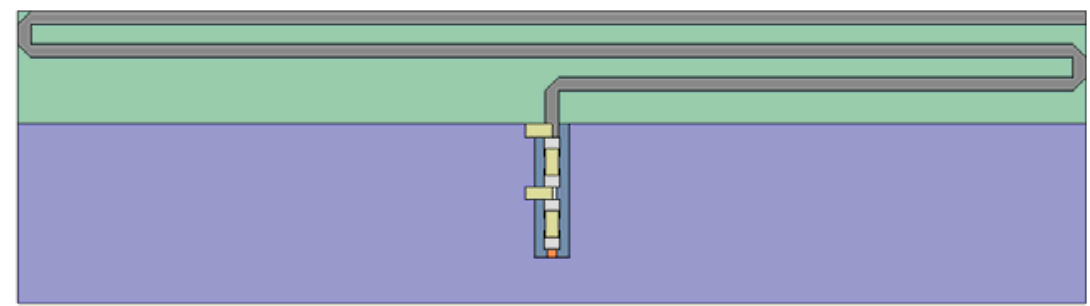

(d)

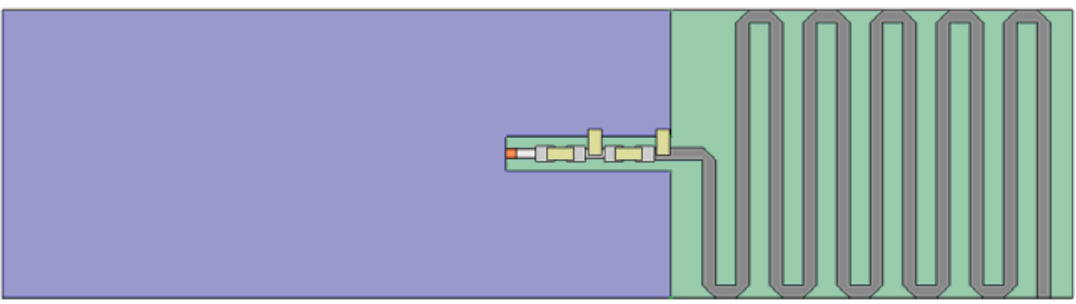

(e)

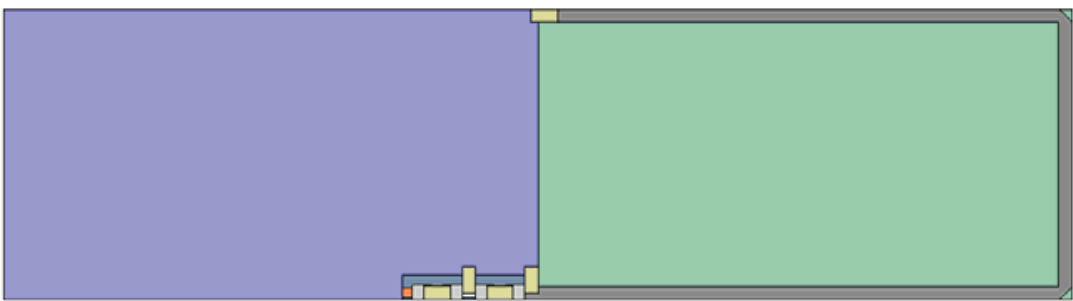

Figure 3.1.1: Ansoft HFSS simulation models (top view) for all antennas. From top-to-bottom: (a) Inverted-F antenna, (b) Long meandering-monopole antenna, (c) Short meanderingmonopole antenna, (d) Compact meandering-monopole antenna, and (e) Loop antenna. 


\subsection{Models (Isometric View)}

(a)

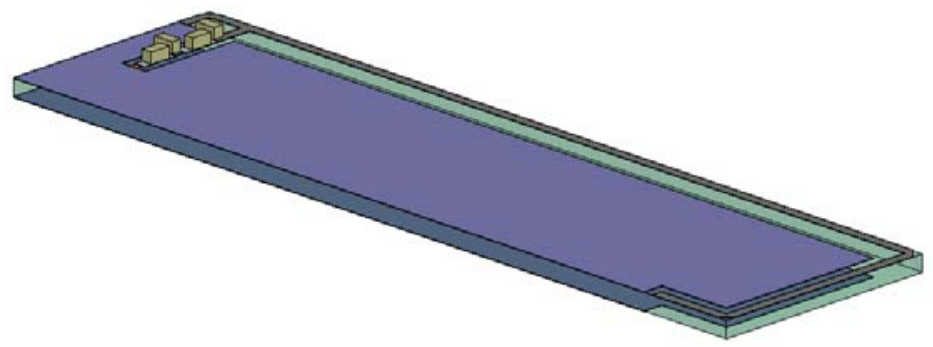

(b)

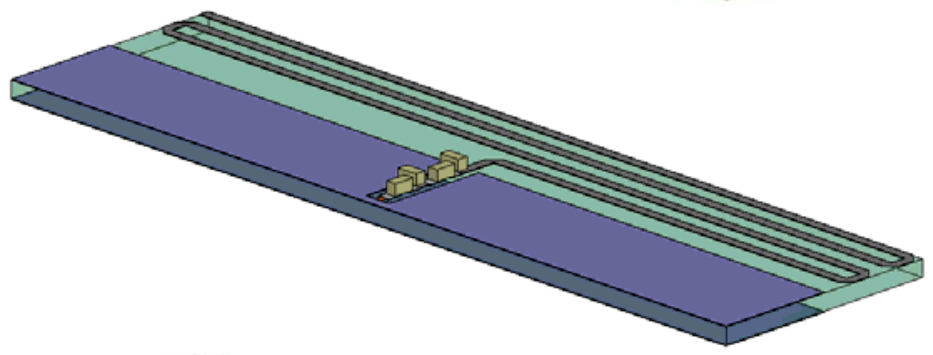

(c)

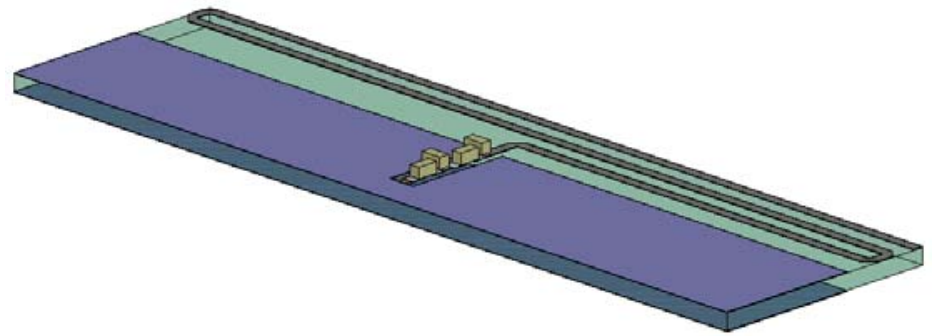

(d)

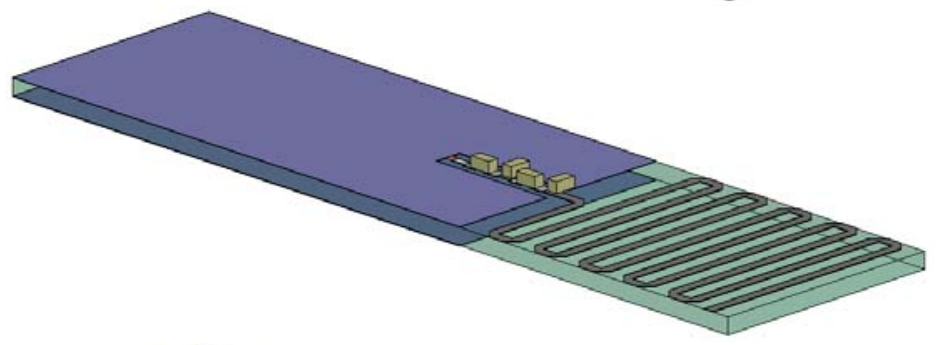

(e)

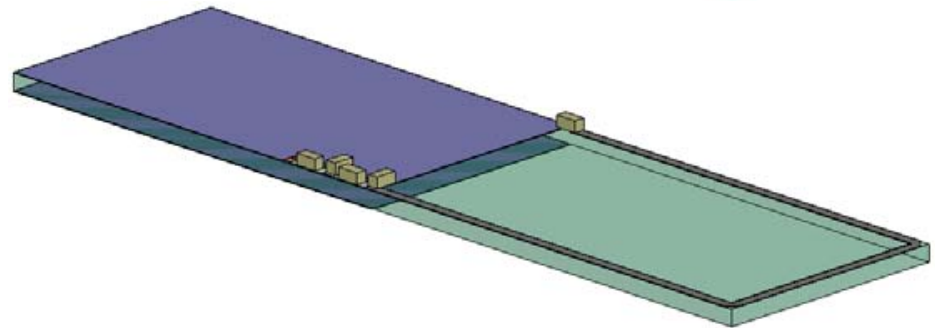

Figure 3.2.1: Ansoft HFSS simulation models (isometric view) for all antennas. From top-tobottom: (a) Inverted-F antenna, (b) Long meandering-monopole antenna, (c) Short meanderingmonopole antenna, (d) Compact meandering-monopole antenna, and (e) Loop antenna. 


\subsection{Inverted-F Antenna Simulation}

\subsubsection{Antenna Orientation and Matching Circuit}

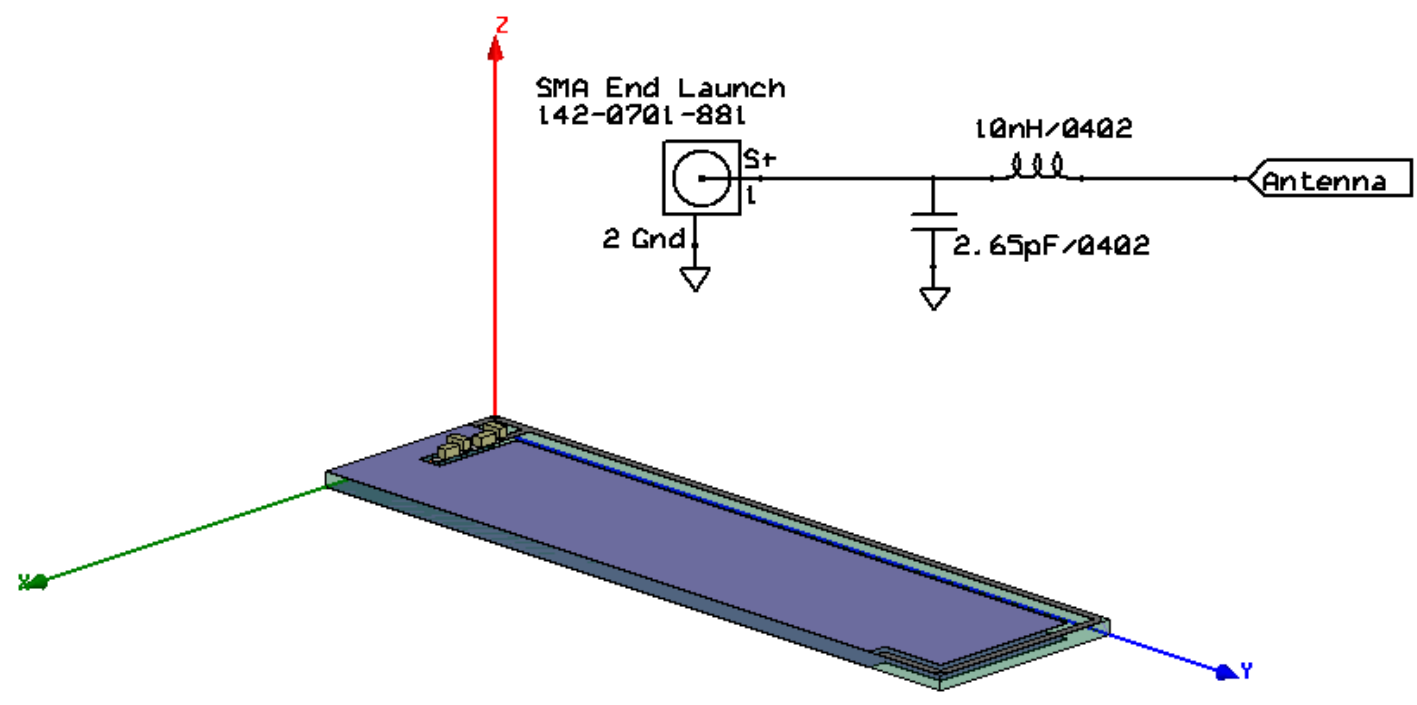

Figure 3.3.1: Inverted-F antenna isometric orientation and simulated matching network required for 50-Ohm match.

\subsubsection{Simulated 3D Radiation Pattern Gain (dB) after Matching}
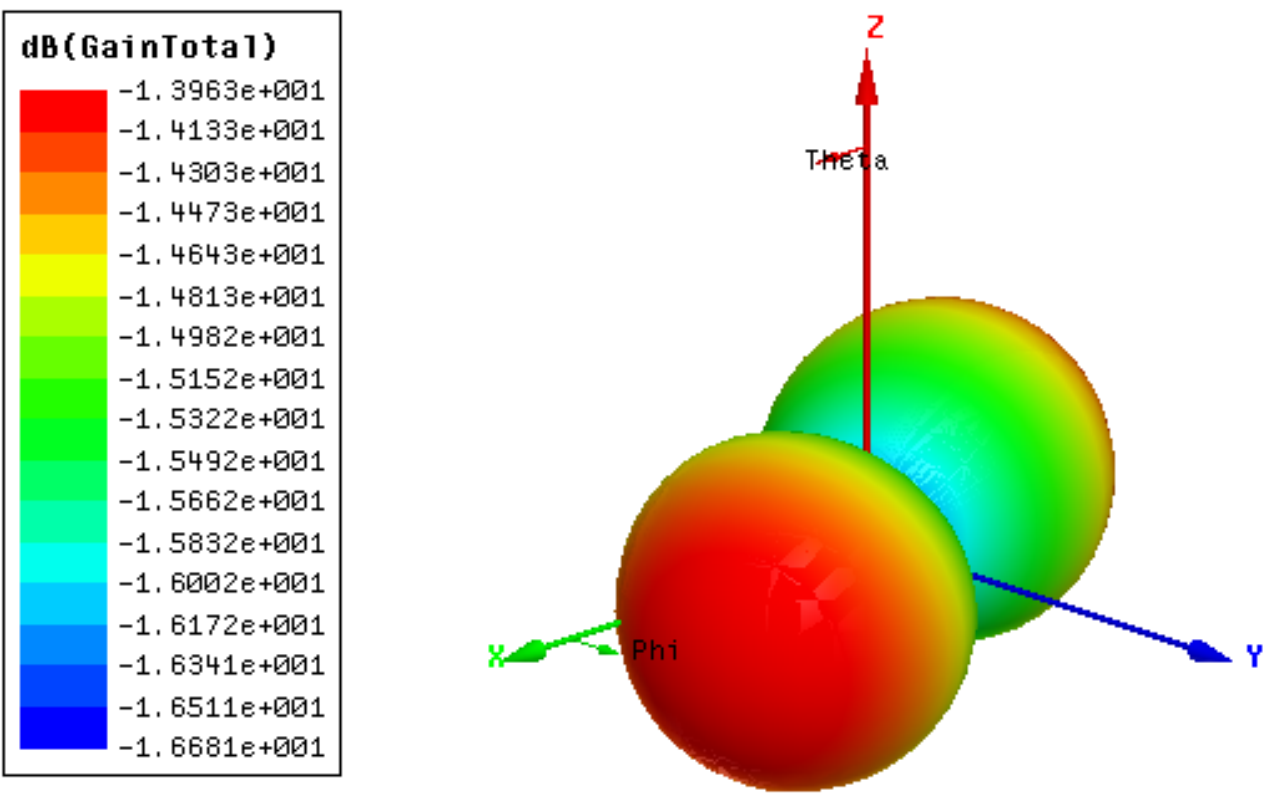

Figure 3.3.2: Inverted-F antenna simulated 3D-radiation pattern expressed as gain in $\mathrm{dB}$ for the isometric orientation before addition of a matching network. 


\subsubsection{Simulated 2D Radiation Pattern after Matching}
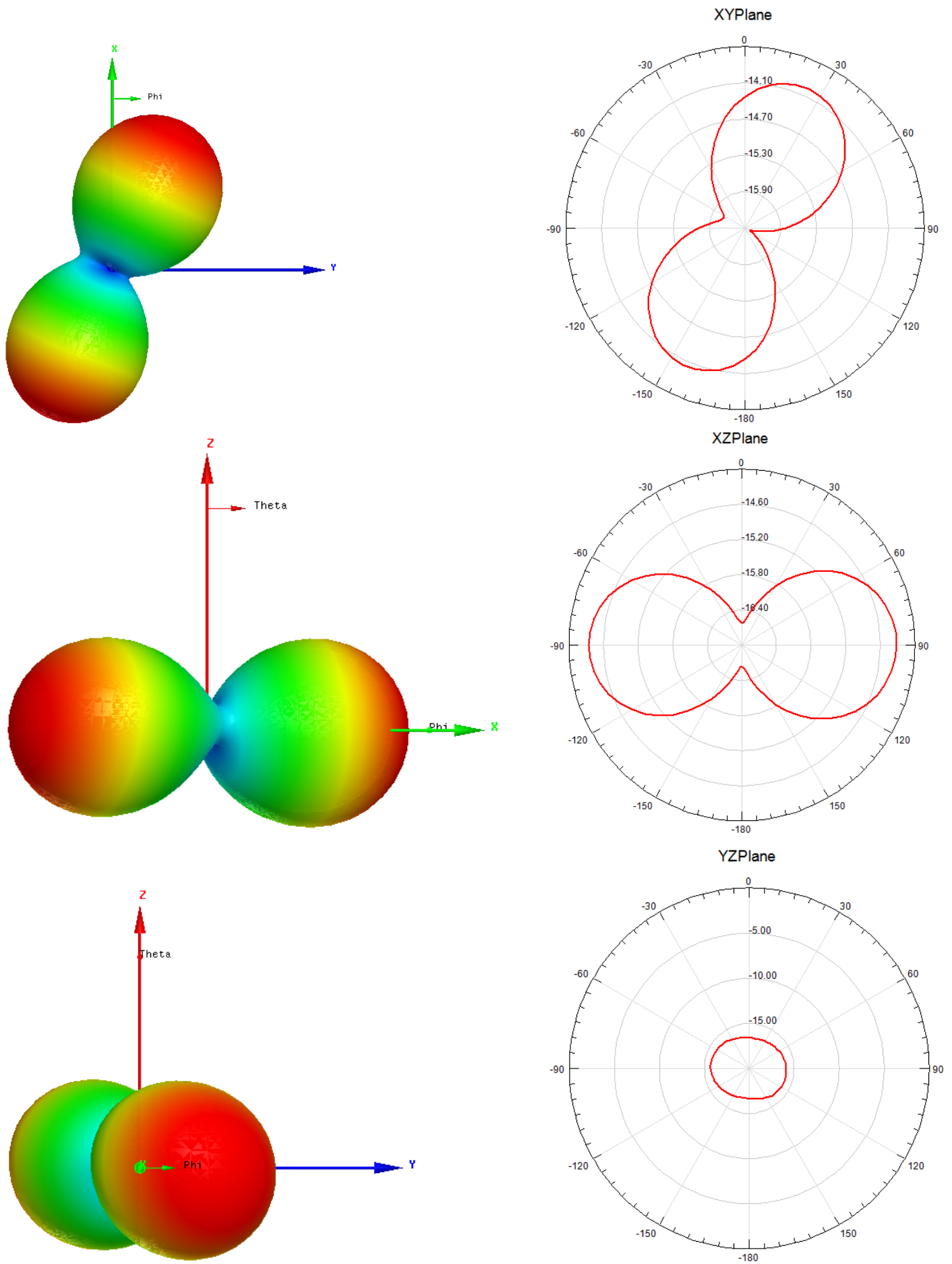

Figure 3.3.3: Inverted-F antenna simulated 2D-radiation pattern expressed as gain in $\mathrm{dB}$ for $\mathrm{XY}$, $\mathrm{XZ}$, and $\mathrm{YZ}$ planes before addition of a matching network. 


\subsubsection{Simulated S11 Reflection Coefficient (dB)}

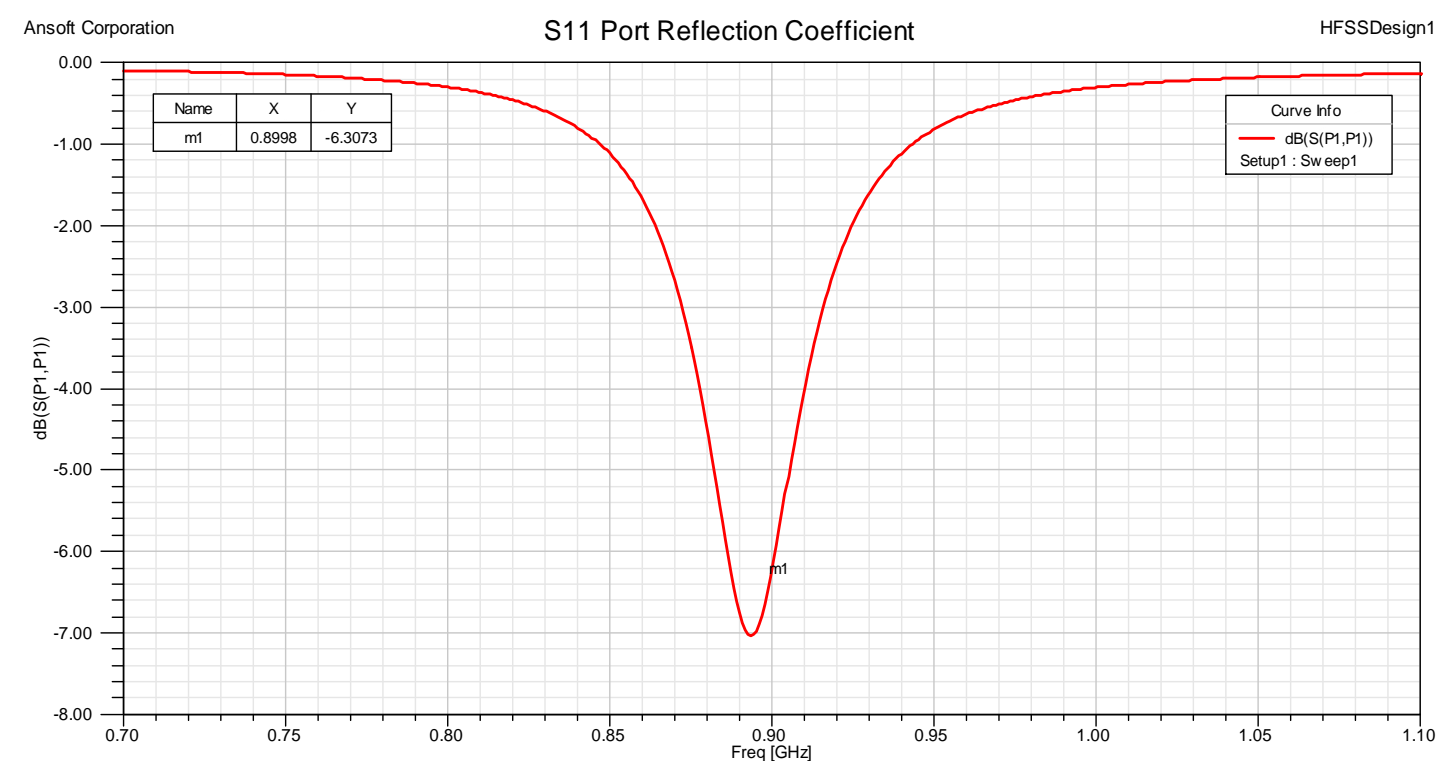

Figure 3.3.4: Inverted-F antenna input-port reflection coefficient (S11) expressed in dB before addition of a matching network.

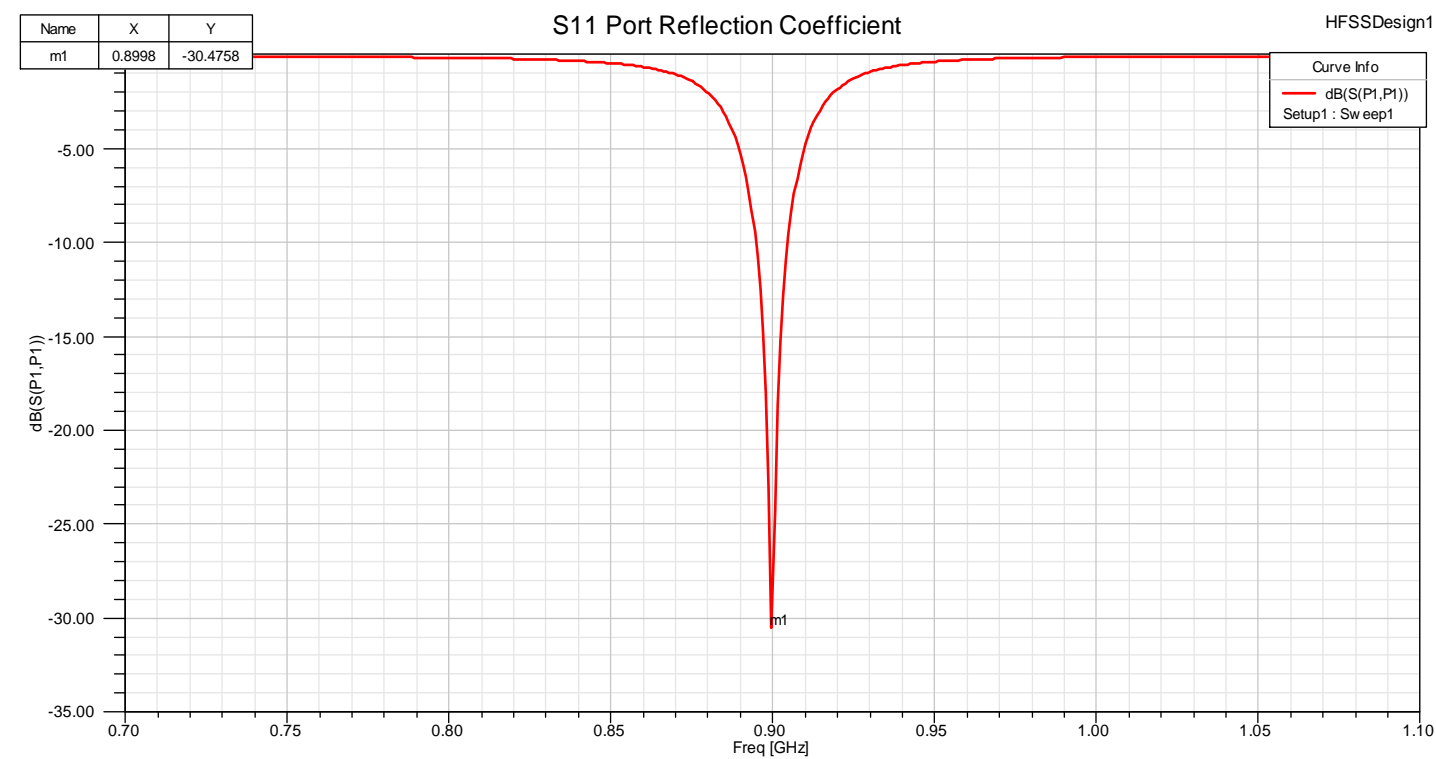

Figure 3.3.5: Inverted-F antenna input-port reflection coefficient (S11) expressed in dB after addition of a matching network. 


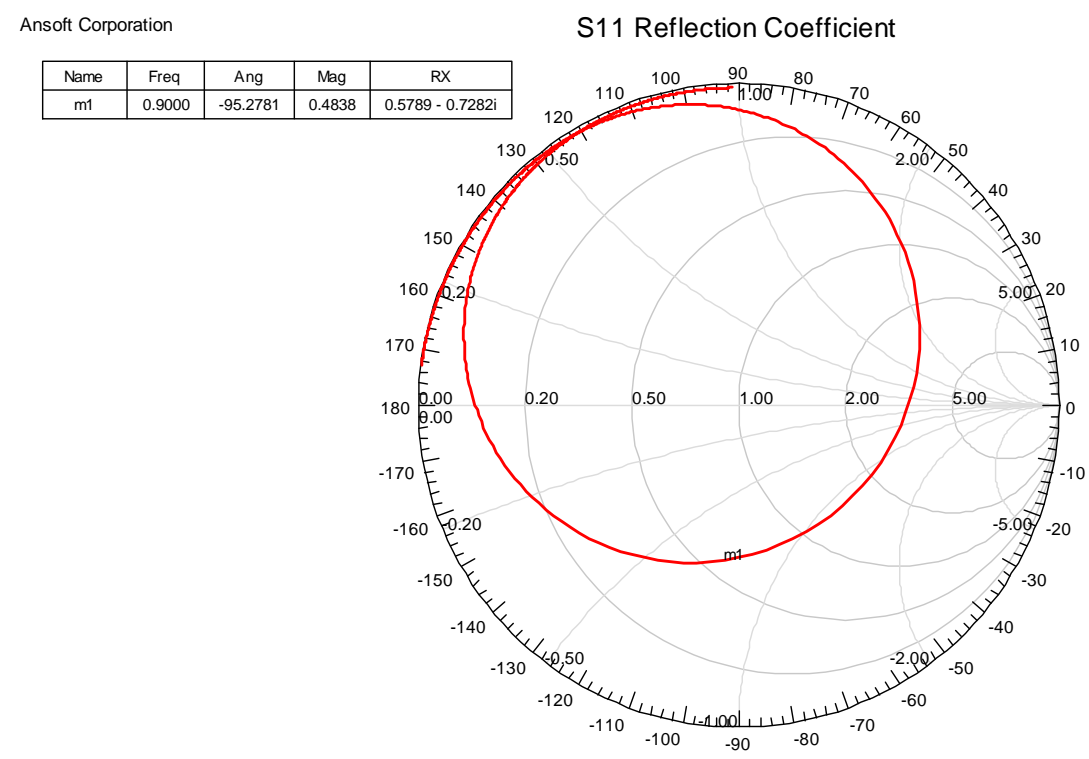

HFSSDesign1

Figure 3.3.6: Inverted-F antenna input-port reflection coefficient (smith chart) before addition of a matching network.

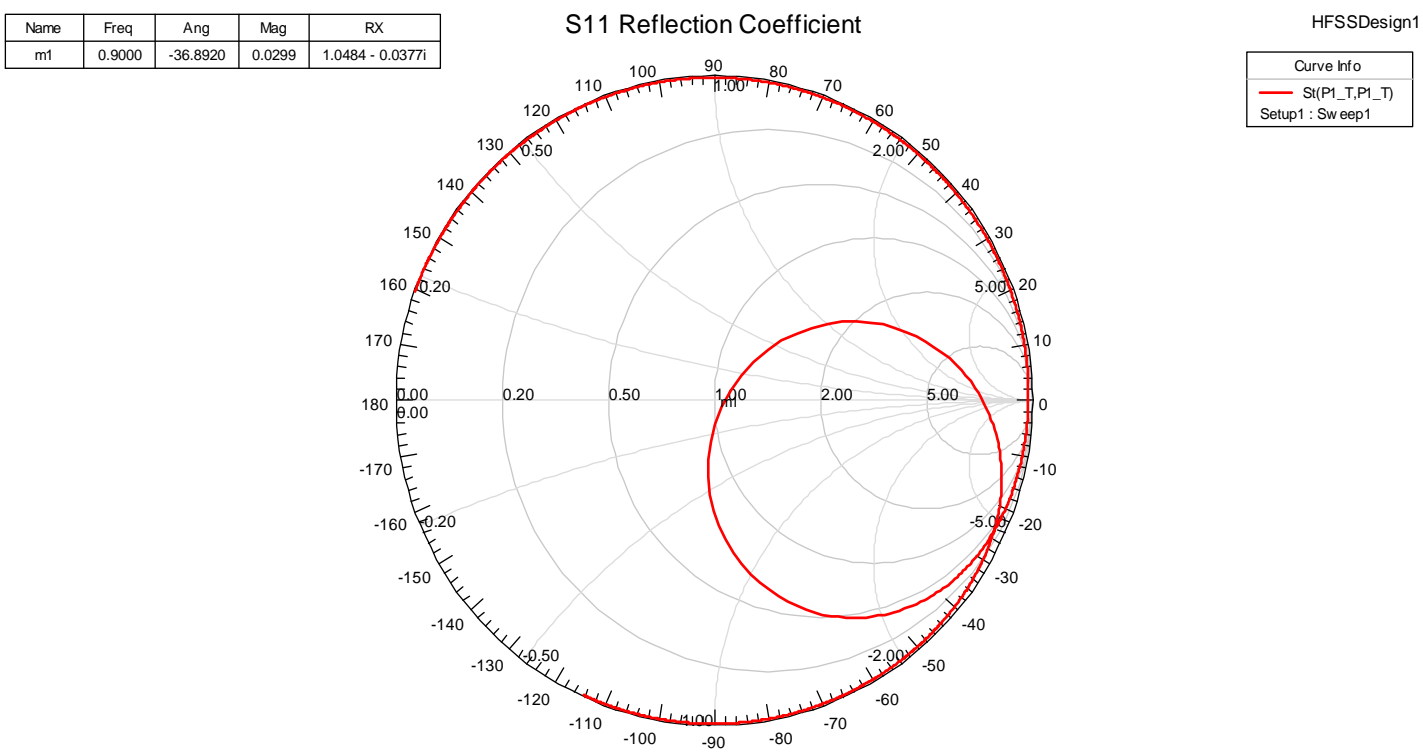

Figure 3.3.7: Inverted-F antenna input-port reflection coefficient (smith chart) after addition of a matching network. 


\subsubsection{Simulated Voltage Standing Wave Ratio (VSWR)}

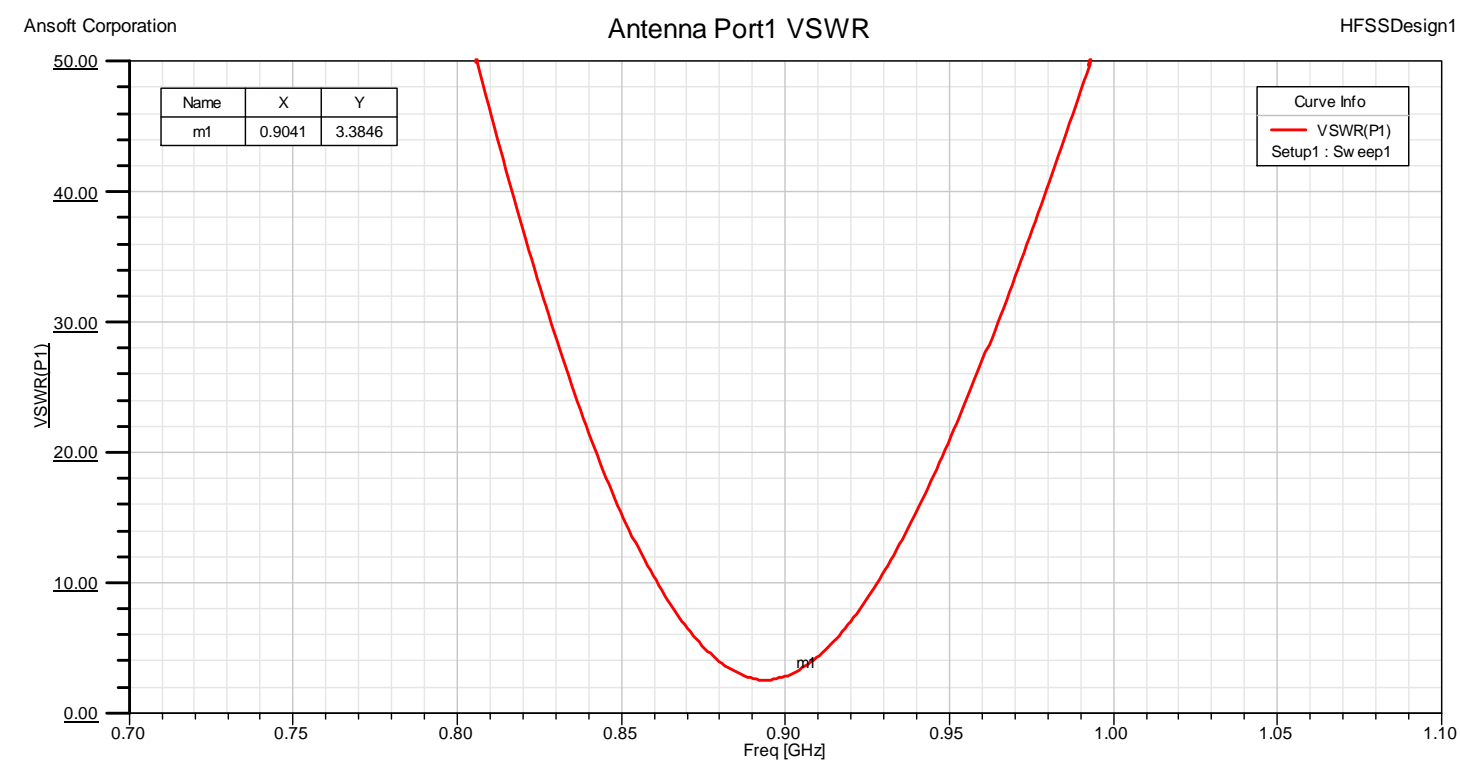

Figure 3.3.8: Inverted-F antenna input-port Voltage Standing Wave Ratio (VSWR) before addition of a matching network.

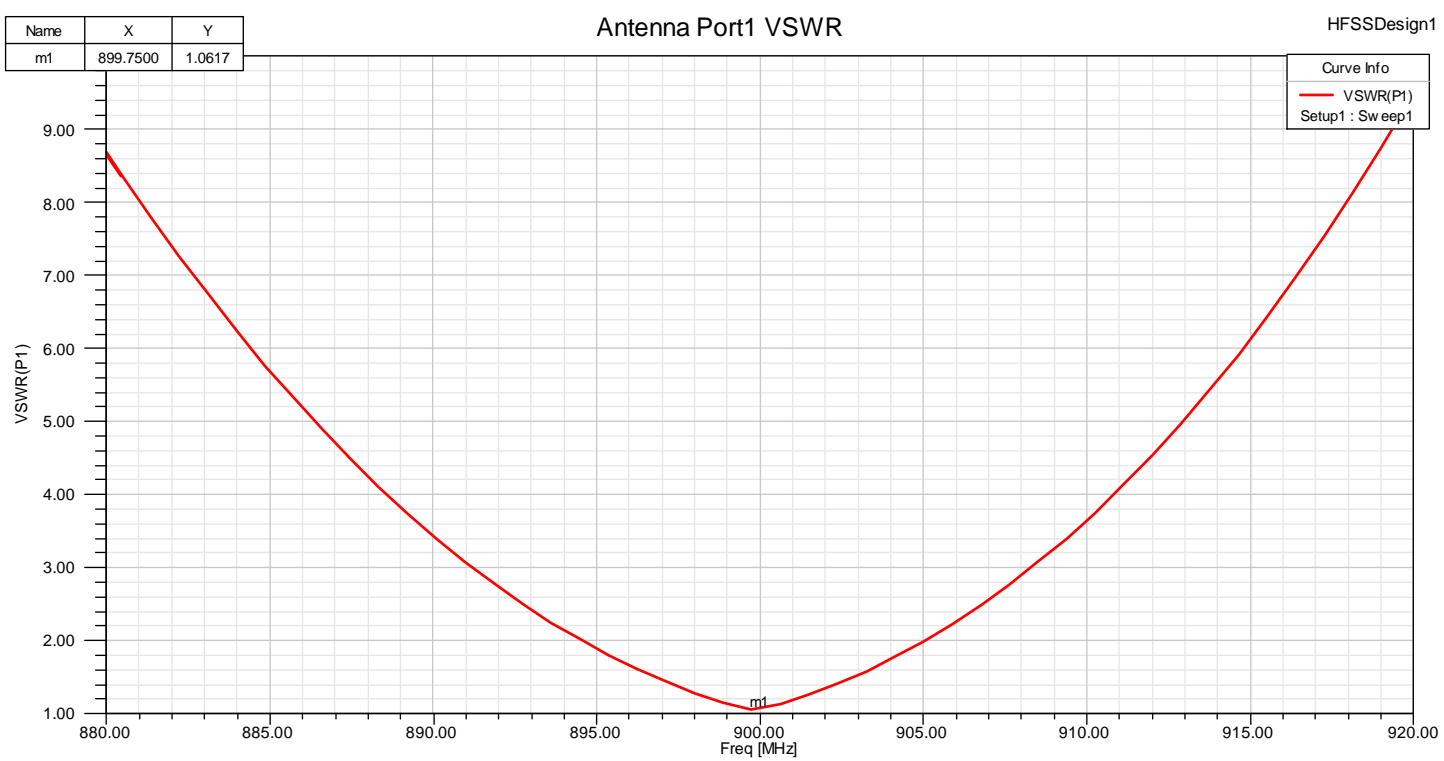

Figure 3.3.9: Inverted-F antenna input-port Voltage Standing Wave Ratio (VSWR) after addition of a matching network. 


\subsection{Long Meandering Monopole Simulation}

\subsubsection{Antenna Orientation and Matching Circuit}

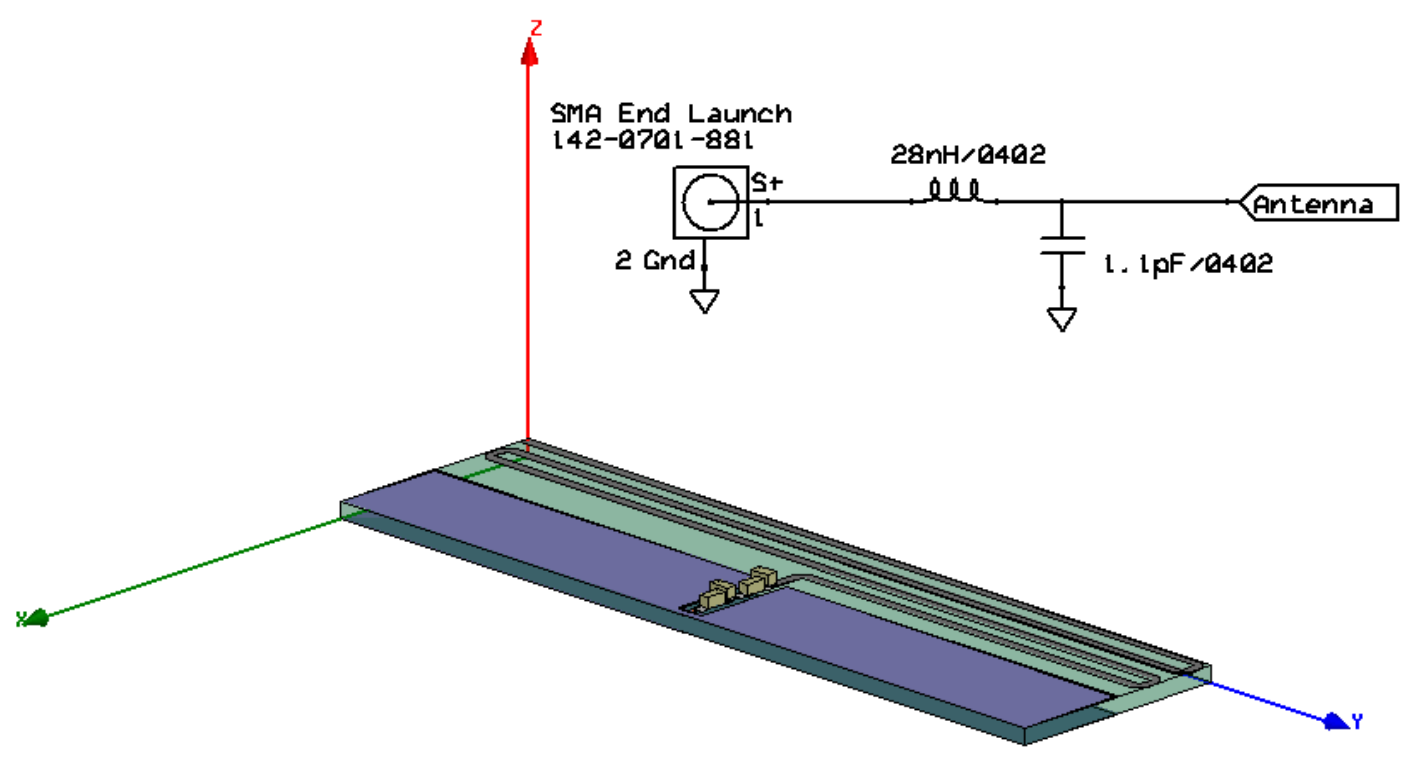

Figure 3.4.1: Long meandering-monopole isometric orientation and simulated matching network required for 50-0hm match.

\subsubsection{Simulated 3D Radiation Pattern Gain (dB) after Matching}
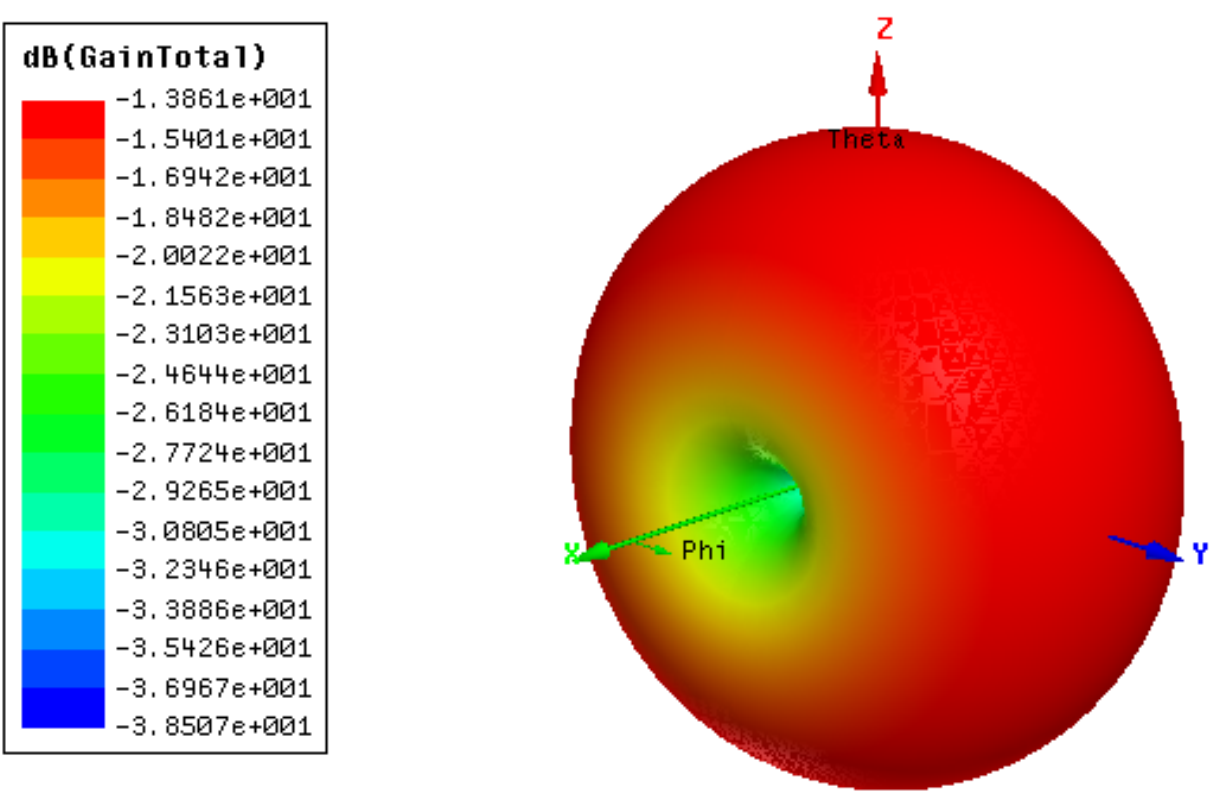

Figure 3.4.2: Long meandering-monopole simulated 3D-radiation pattern expressed as gain in $\mathrm{dB}$ for the isometric orientation before addition of a matching network. 


\subsubsection{Simulated 2D Radiation Pattern after Matching}
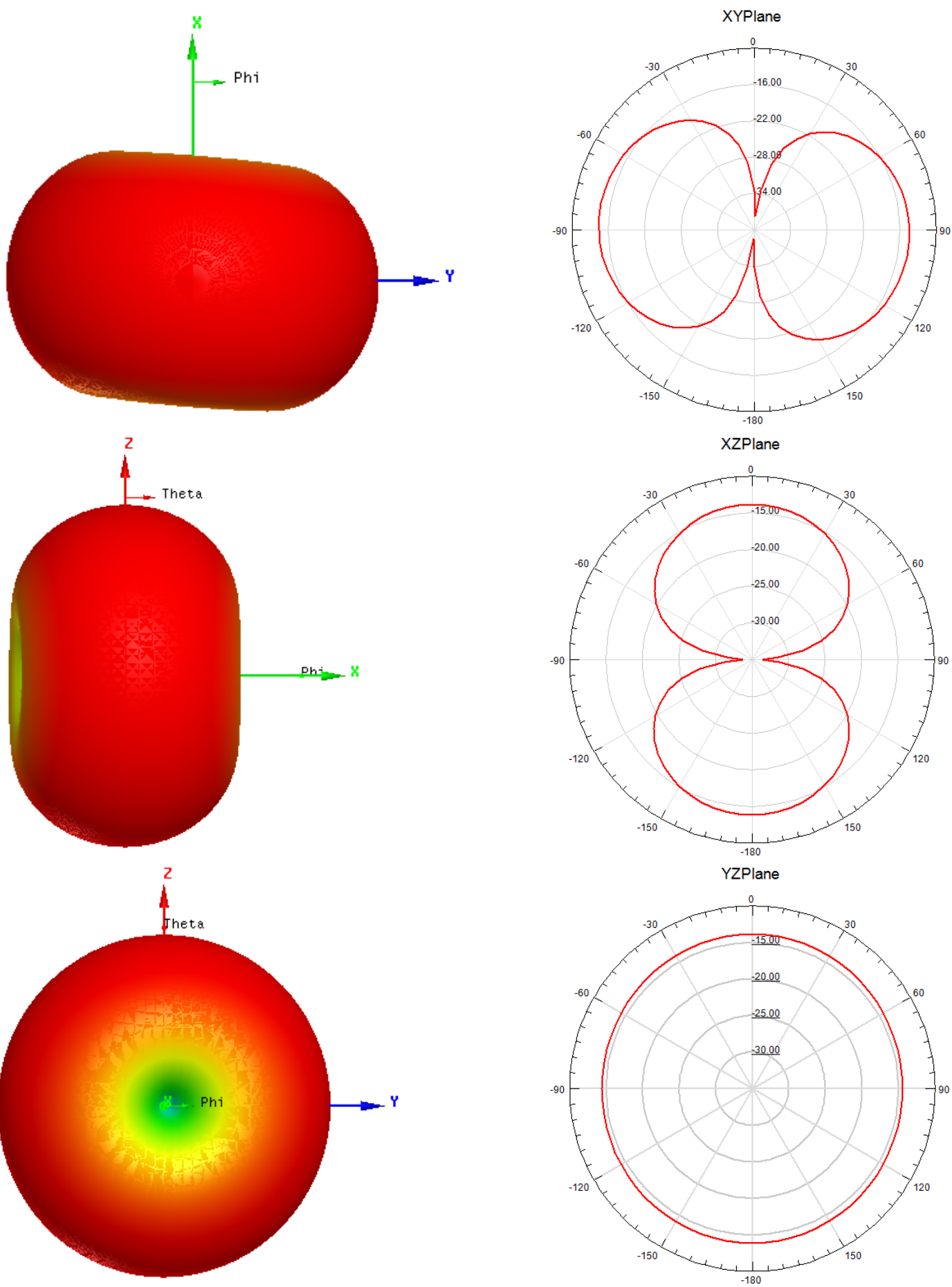

Figure 3.4.3: Long meandering-monopole simulated 2D-radiation pattern expressed as gain in dB for XY, XZ, and YZ planes before addition of a matching network. 


\subsubsection{Simulated S11 Reflection Coefficient (dB)}

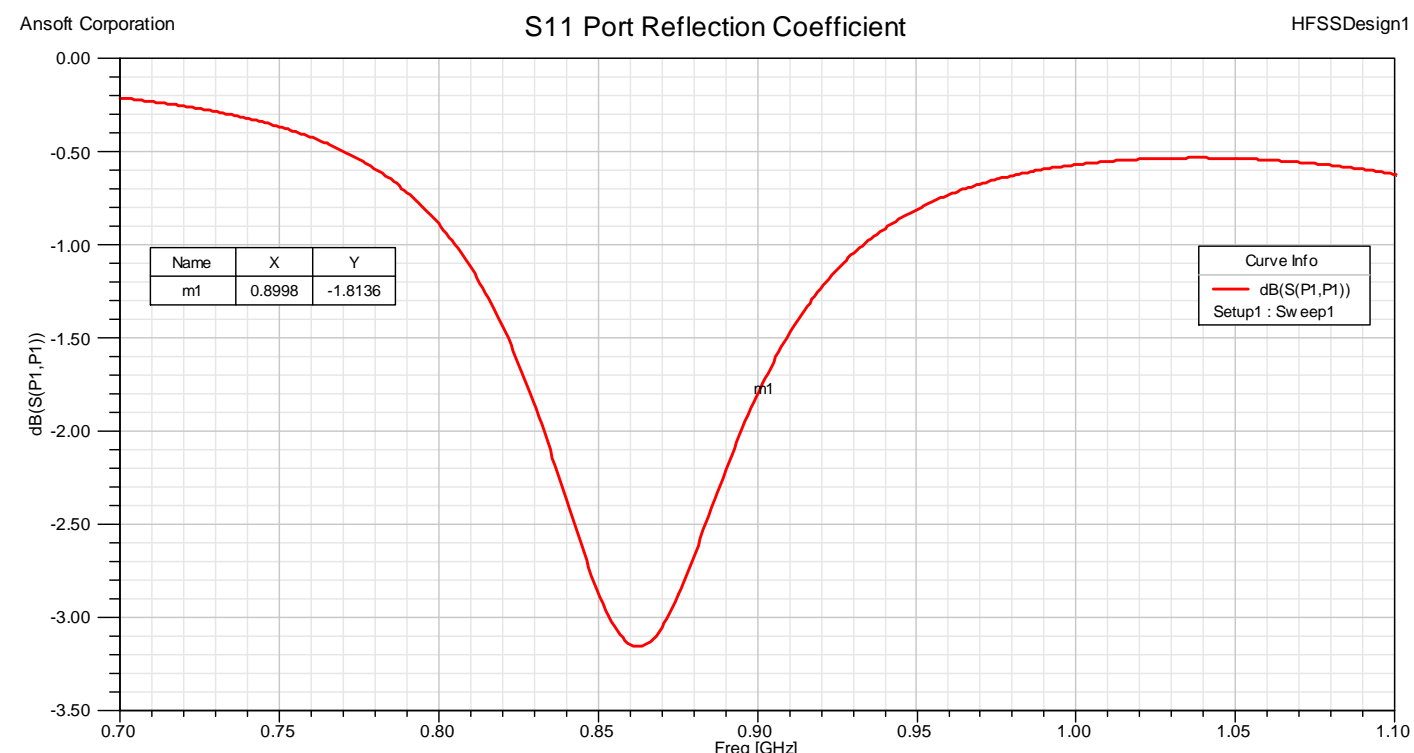

Figure 3.4.4: Long meandering-monopole input-port reflection coefficient (S11) expressed in dB before addition of a matching network.

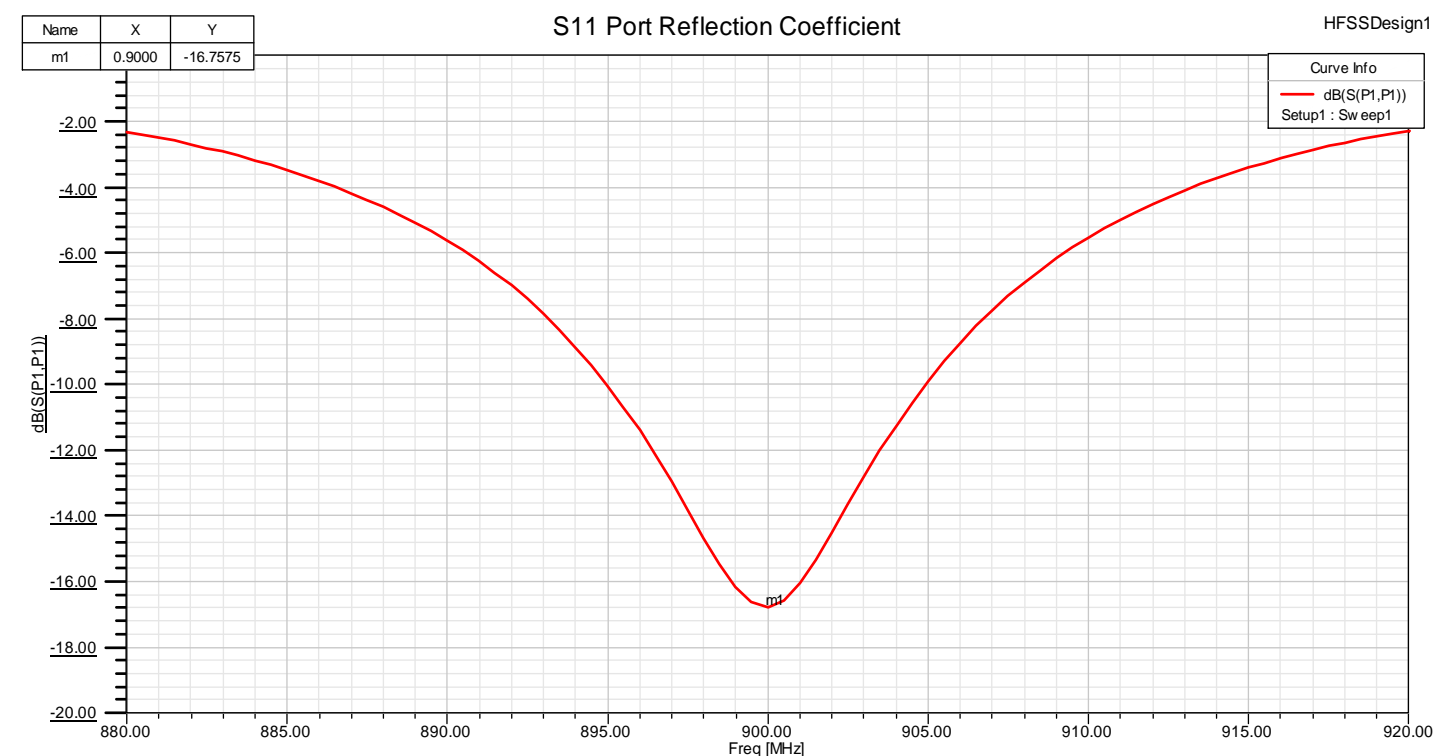

Figure 3.4.5: Long meandering-monopole input-port reflection coefficient (S11) expressed in dB after addition of a matching network. 


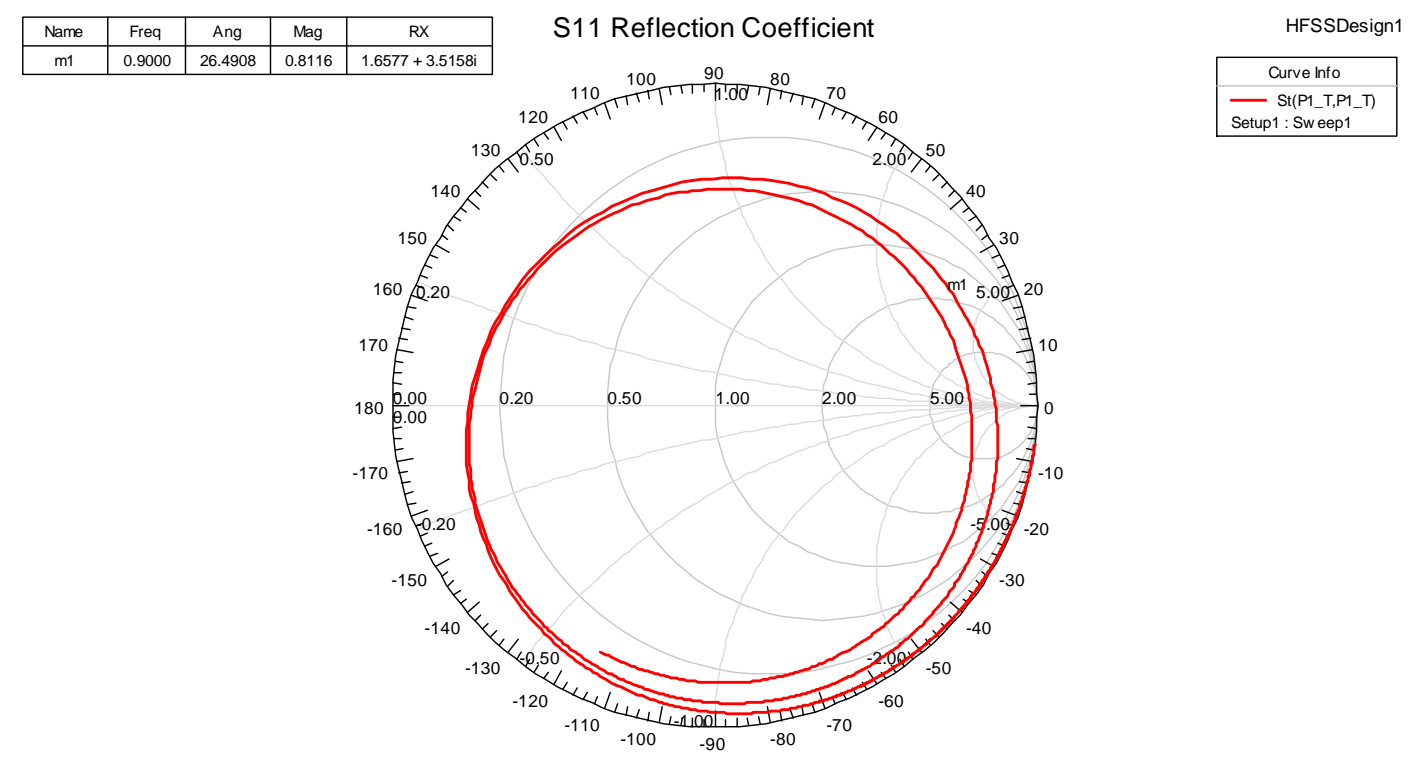

Figure 3.4.6: Long meandering-monopole input-port reflection coefficient (smith chart) before addition of a matching network.
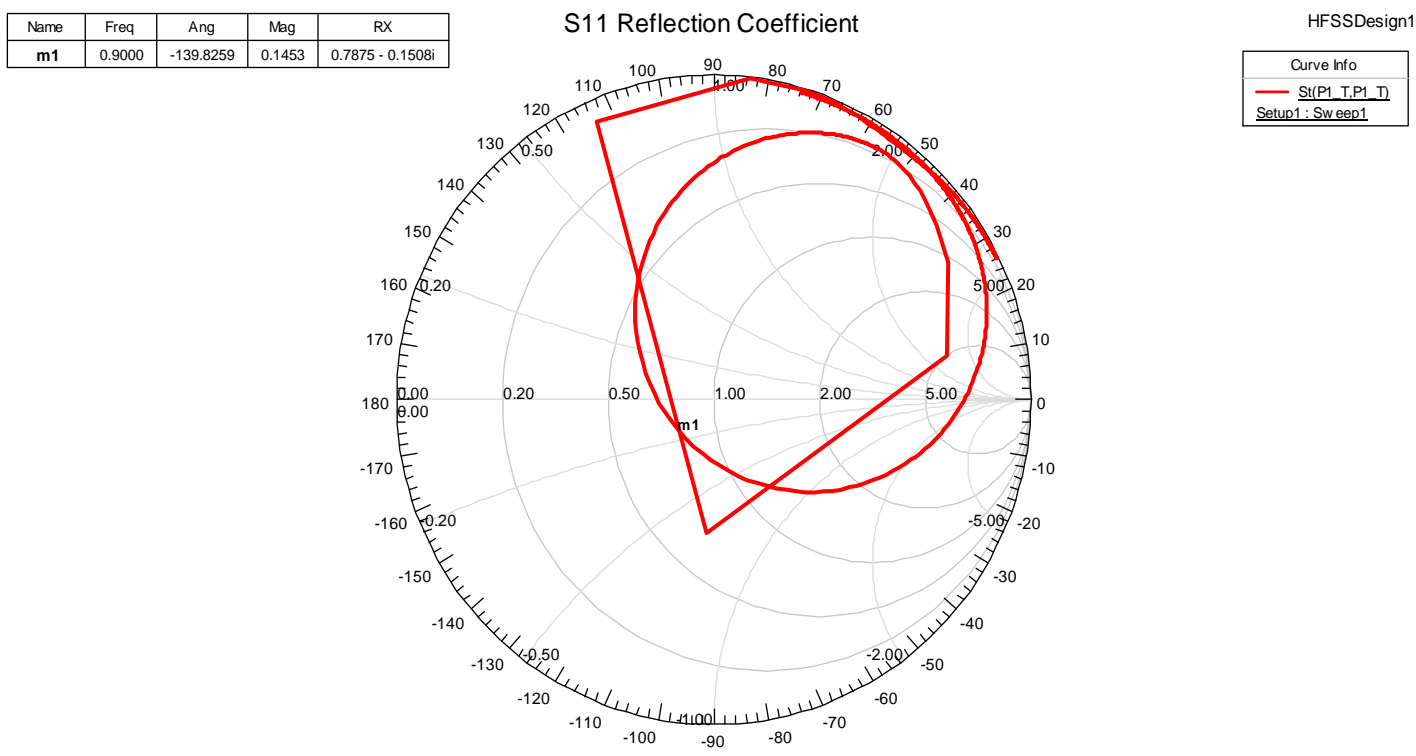

Figure 3.4.7: Long meandering-monopole input-port reflection coefficient (Smith Chart) after addition of a matching network. 


\subsubsection{Simulated Voltage Standing Wave Ratio (VSWR)}

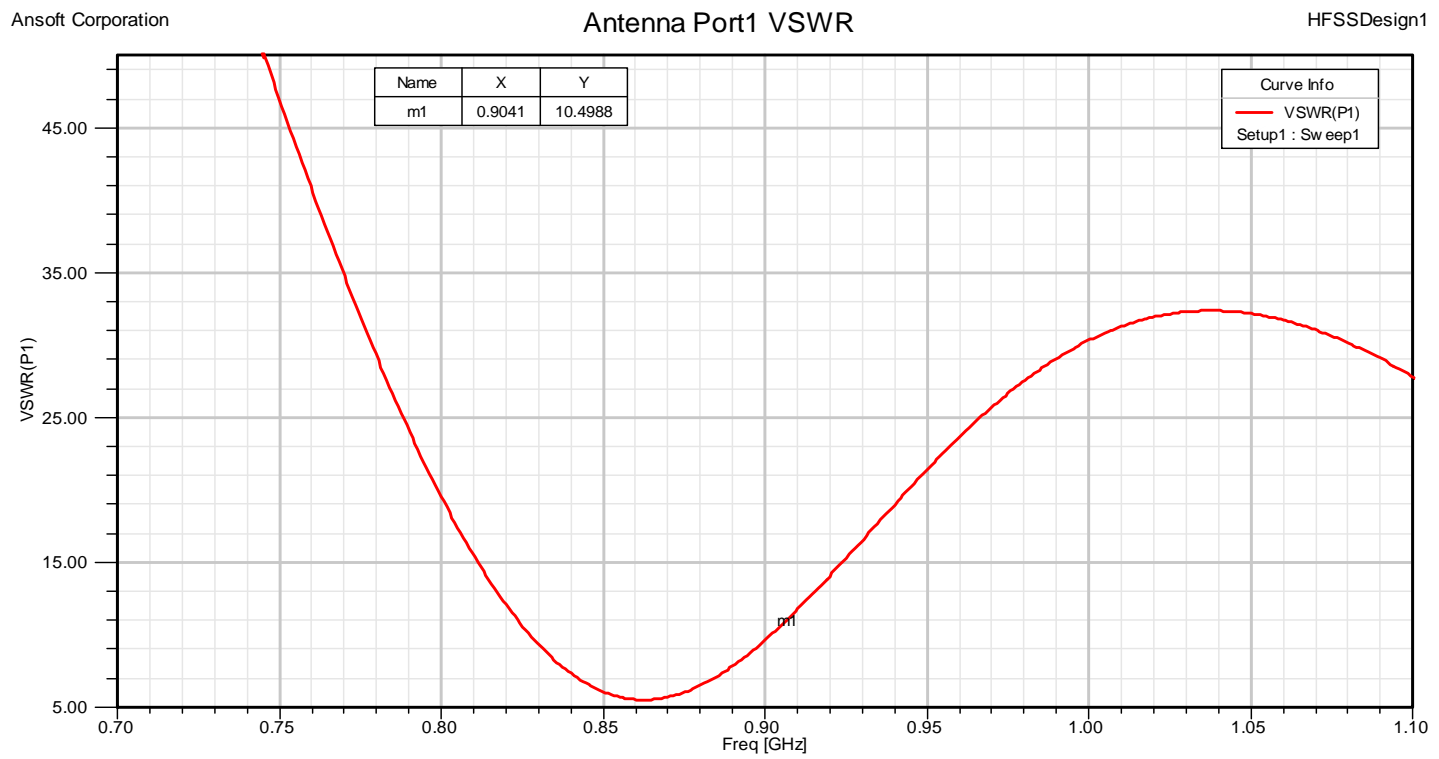

Figure 3.4.8: Long meandering-monopole input-port Voltage Standing Wave Ratio (VSWR) before addition of a matching network.

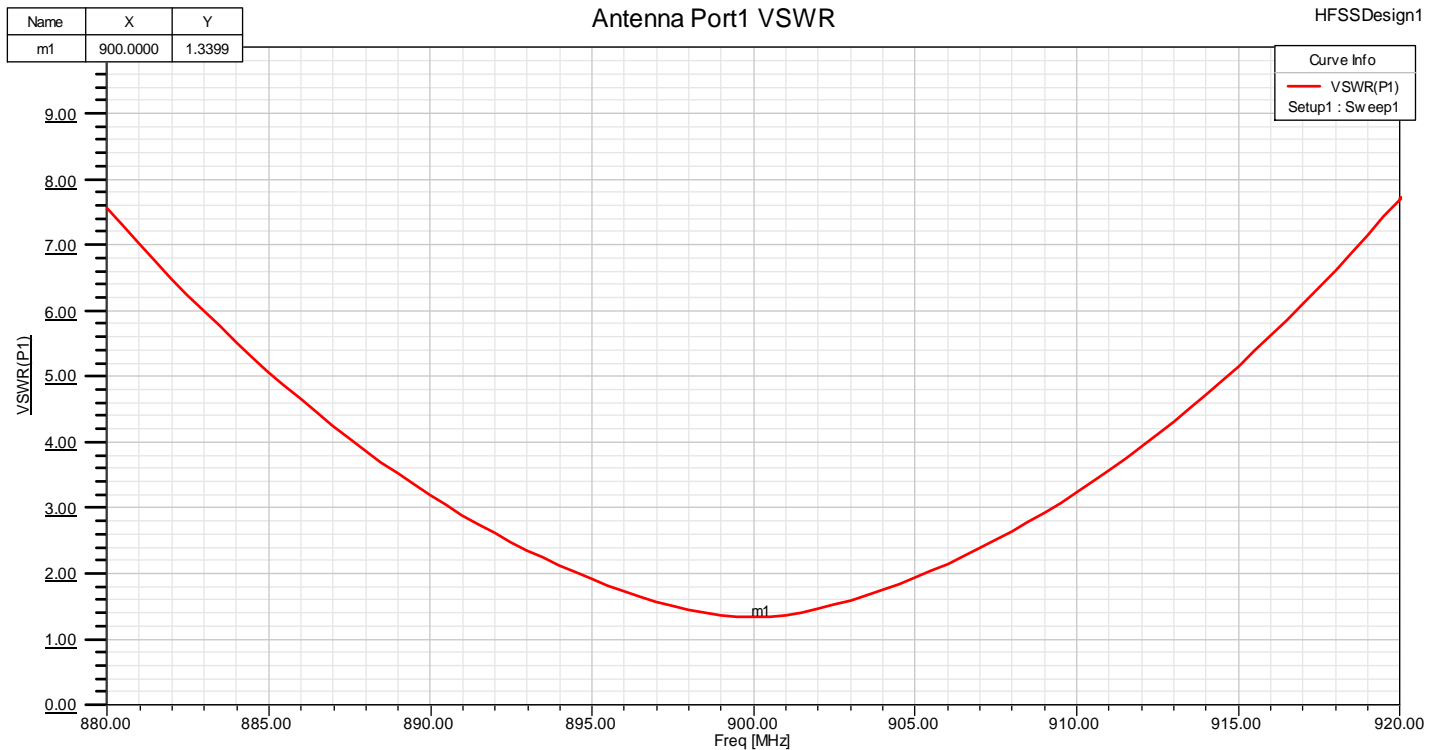

Figure 3.4.9: Long meandering-monopole input-port Voltage Standing Wave Ratio (VSWR) after addition of a matching network. 


\subsection{Short Meandering-Monopole Simulation}

\subsubsection{Antenna Orientation and Matching Circuit}

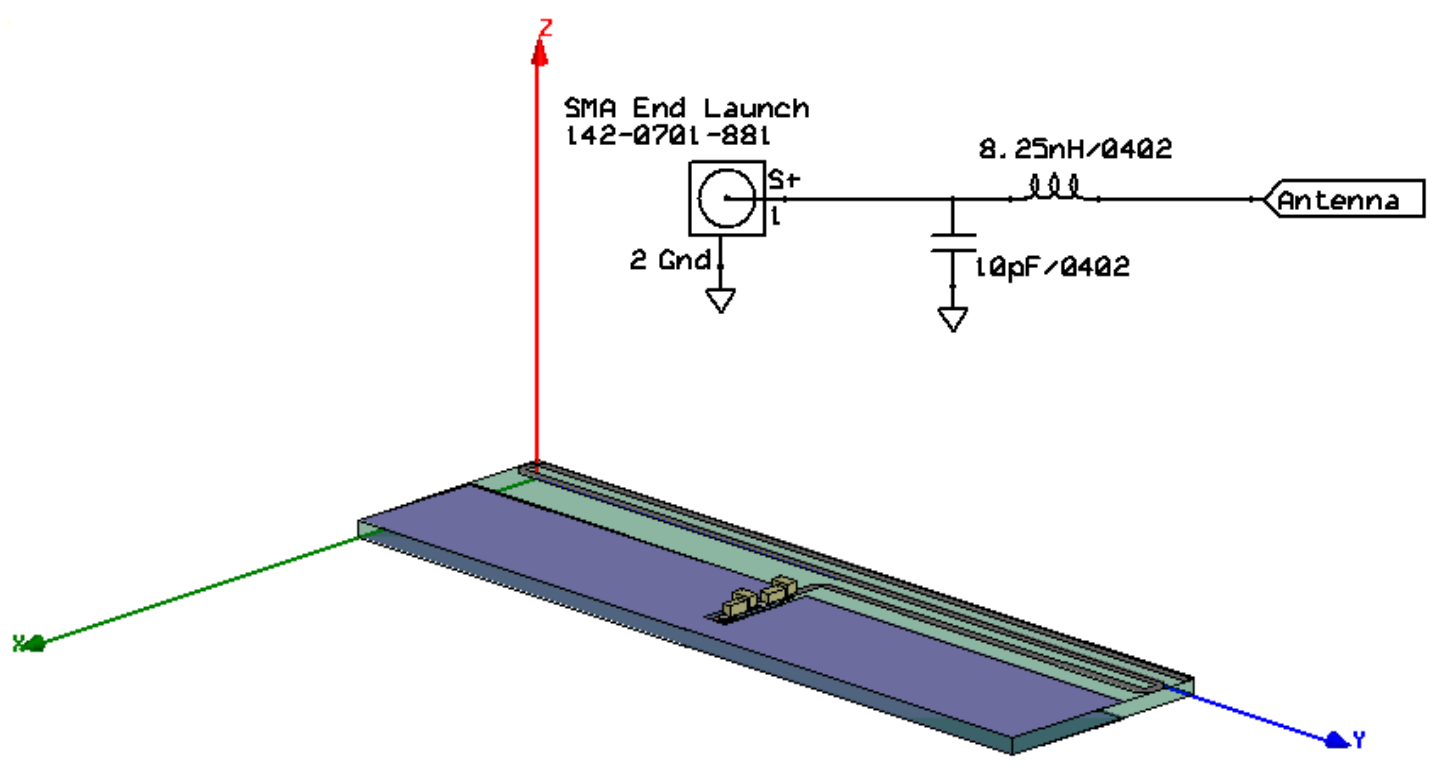

Figure 3.5.1: Short meandering-monopole isometric orientation and simulated matching network required for $50-\mathrm{Ohm}$ match.

\subsubsection{Simulated 3D Radiation Pattern Gain (dB) after Matching}
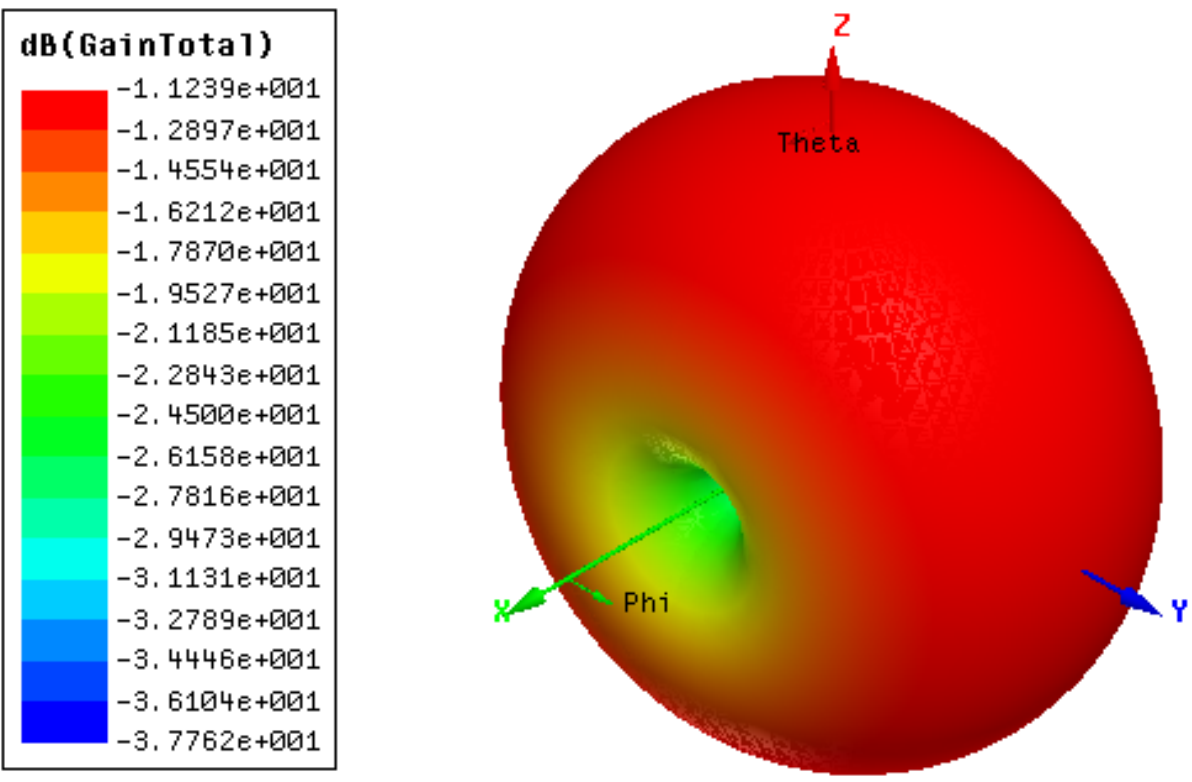

Figure 3.5.2: Short meandering-monopole simulated 3D-radiation pattern expressed as gain in $\mathrm{dB}$ for the isometric orientation before addition of a matching network. 


\subsubsection{Simulated 2D Radiation Pattern}
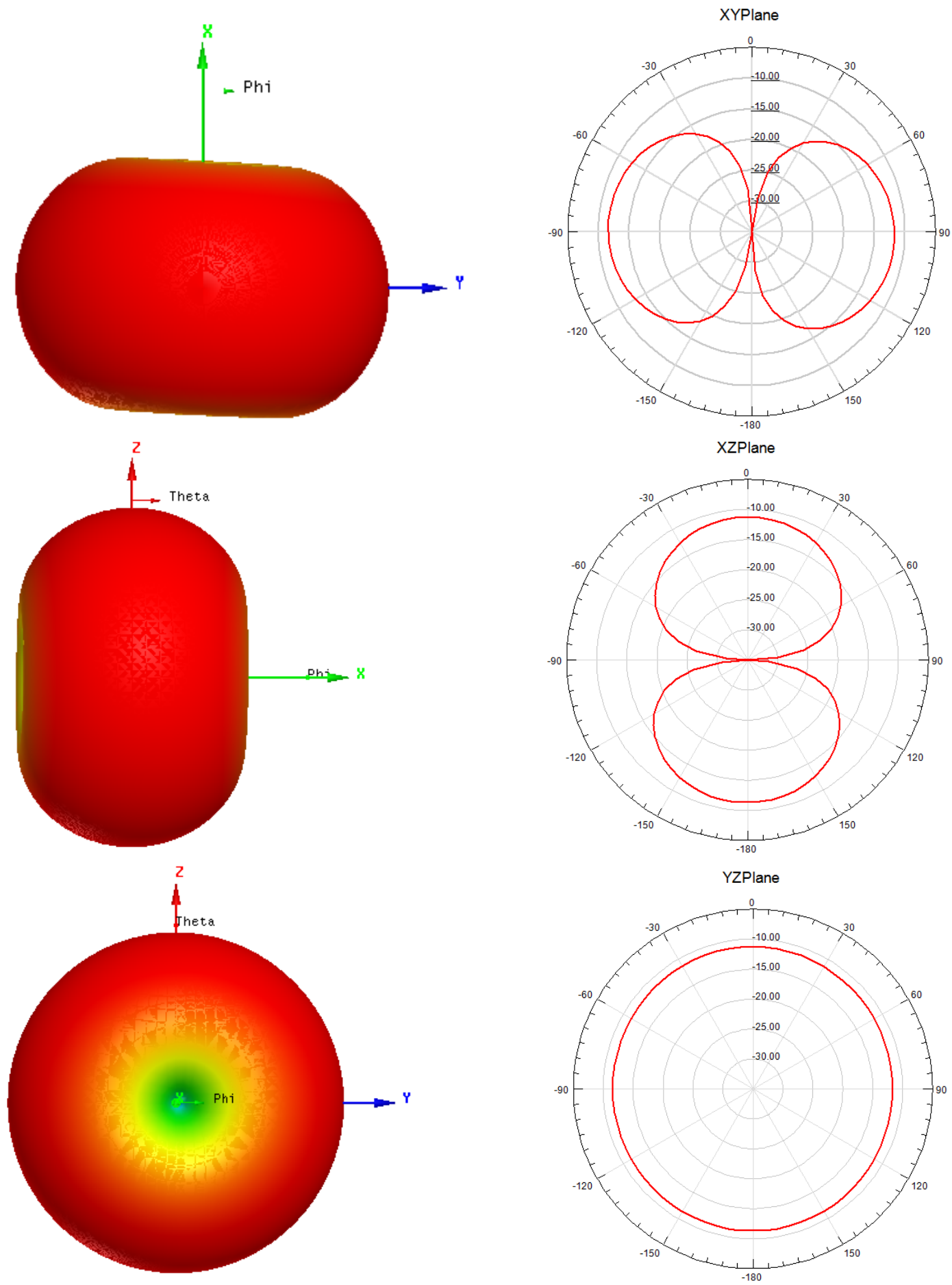

Figure 3.5.3: Short meandering-monopole simulated 2D-radiation pattern expressed as gain in $\mathrm{dB}$ for $\mathrm{XY}, \mathrm{XZ}$, and YZ planes before addition of a matching network. 


\subsubsection{Simulated S11 Reflection Coefficient (dB)}

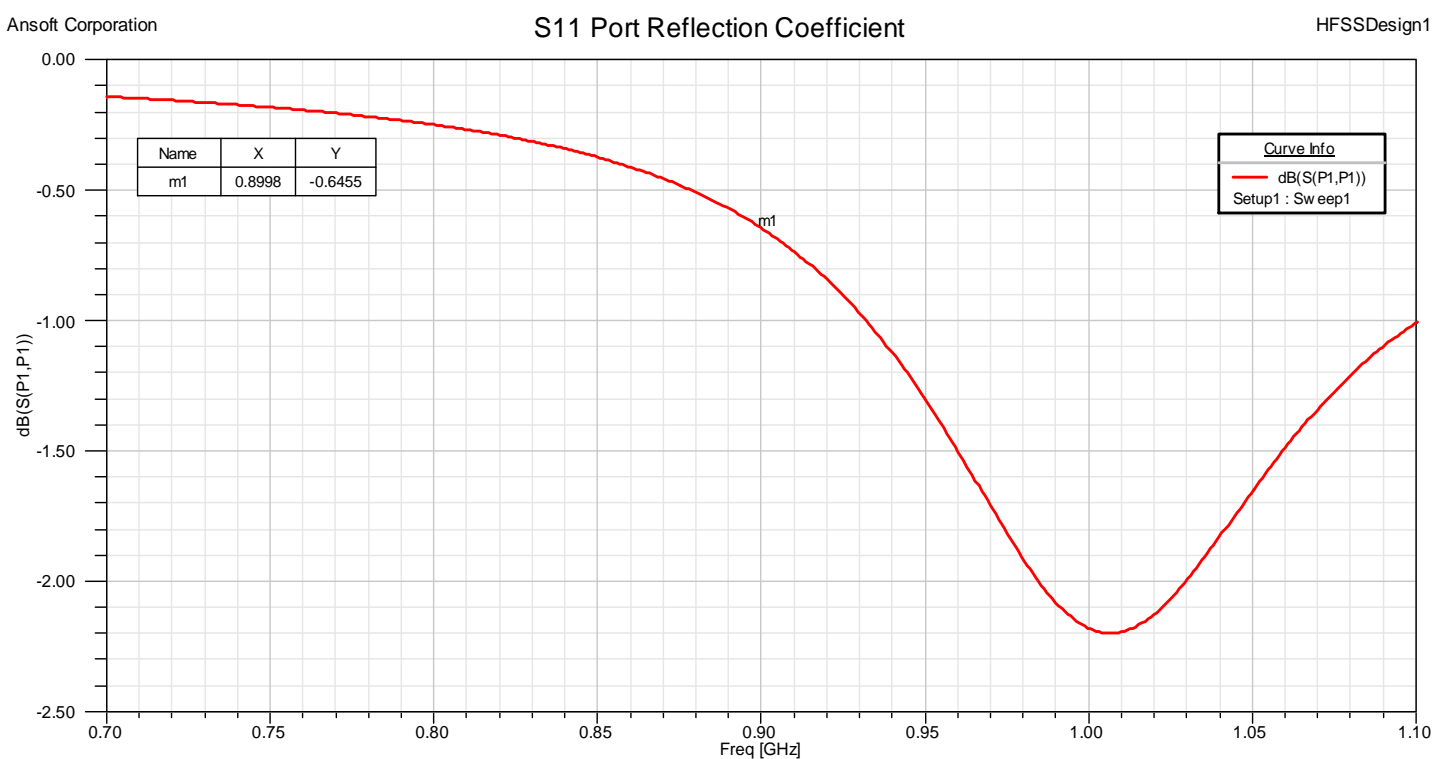

Figure 3.5.4: Short meandering-monopole input-port reflection coefficient (S11) expressed in dB before addition of a matching network.

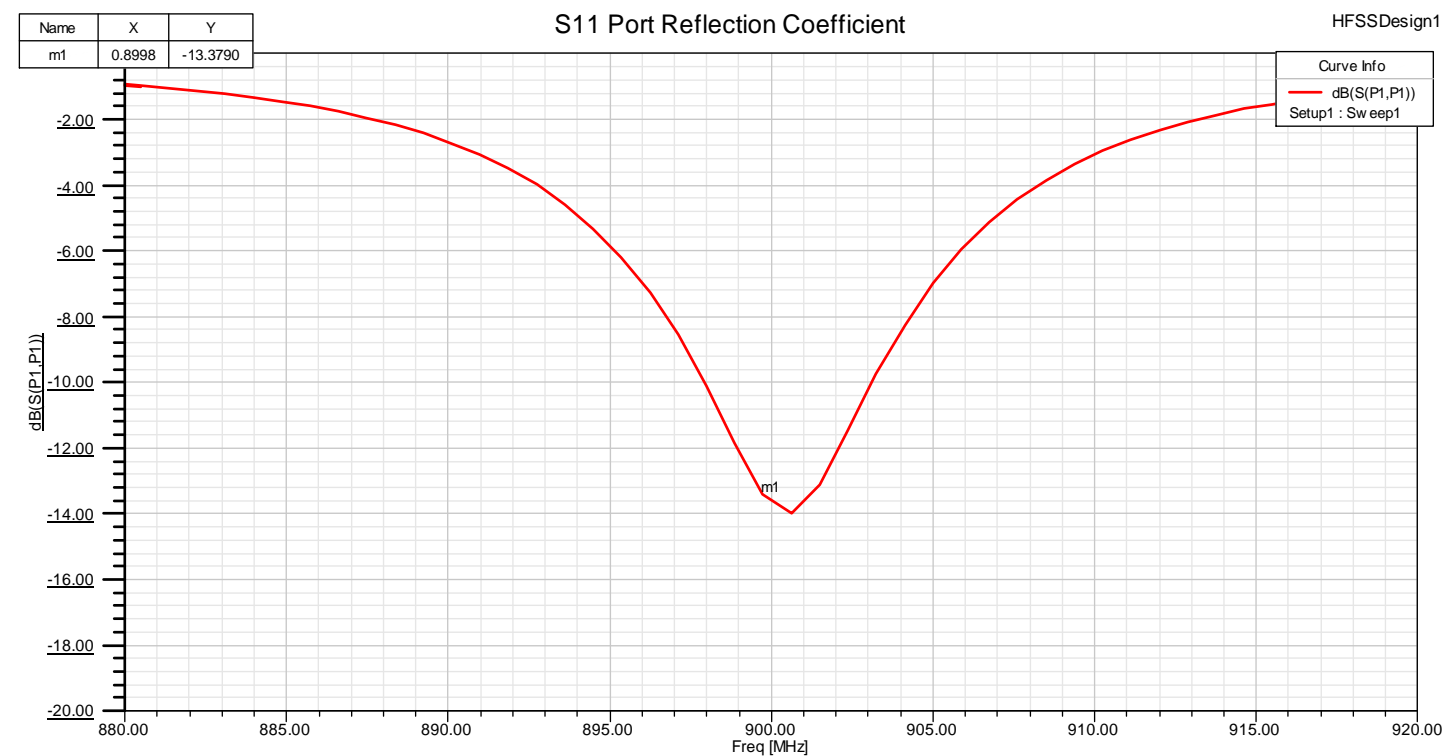

Figure 3.5.5: Short meandering-monopole input-port reflection coefficient (S11) expressed in dB after addition of a matching network.

3 


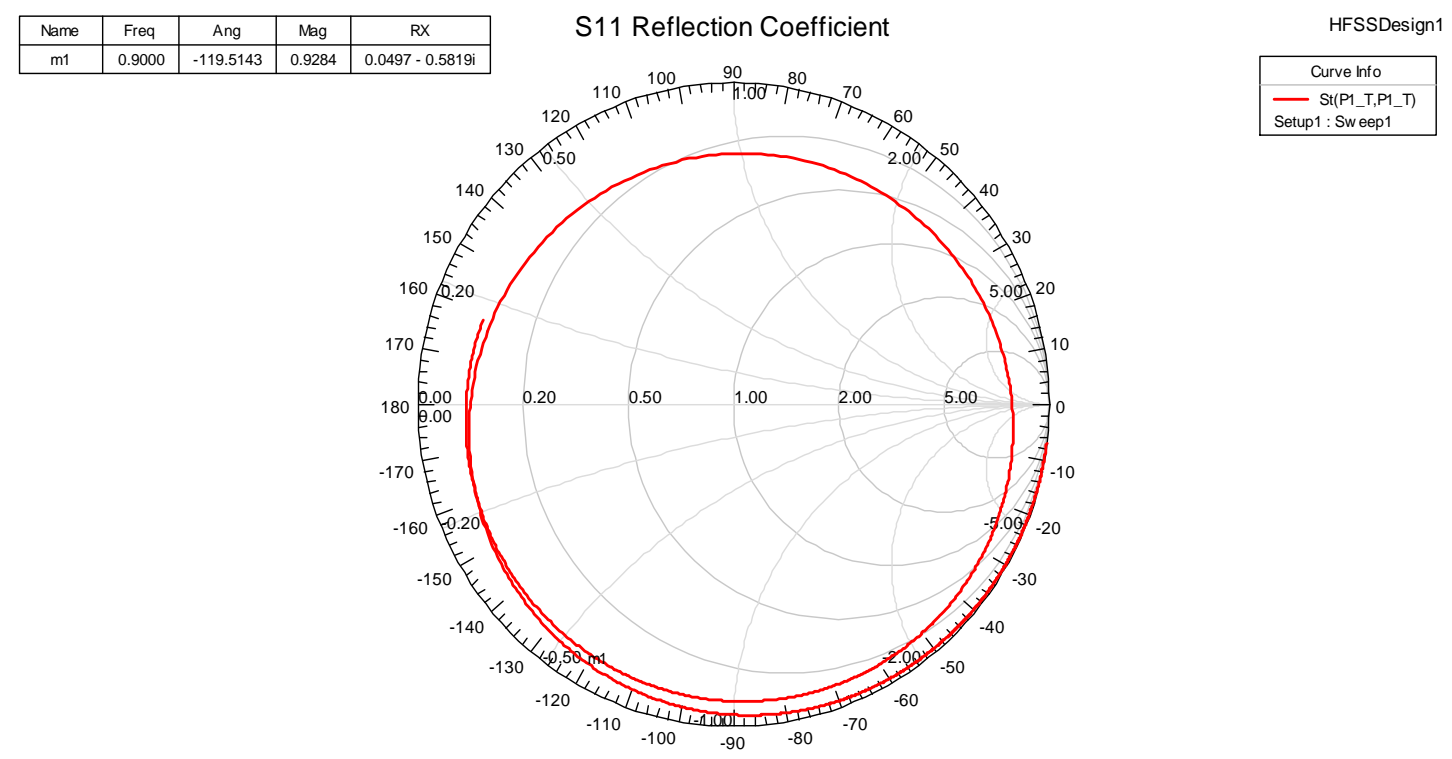

Figure 3.5.6: Short meandering-monopole input-port reflection coefficient (Smith Chart) before addition of a matching network.

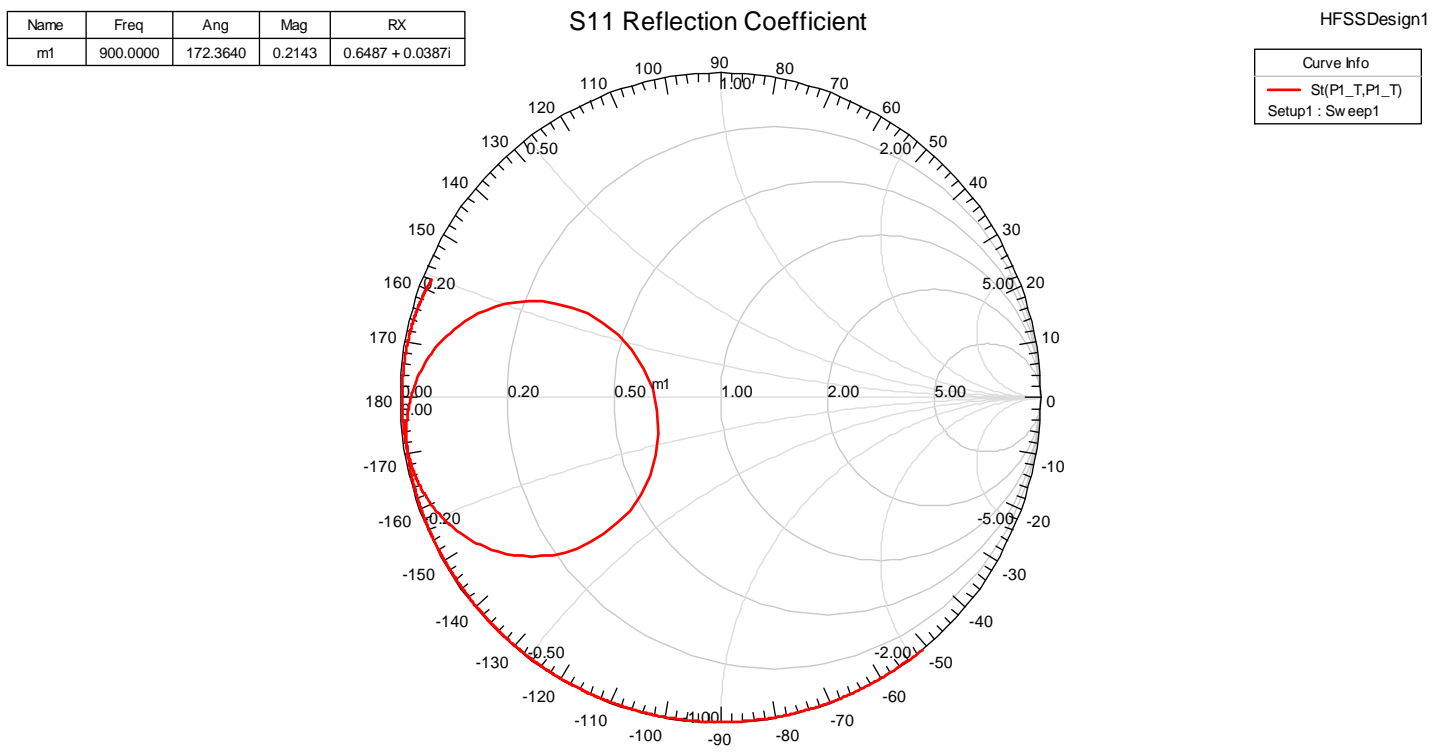

Figure 3.5.7: Short meandering-monopole input-port reflection coefficient (Smith Chart) after addition of a matching network. 


\subsubsection{Simulated Voltage Standing Wave Ratio (VSWR)}

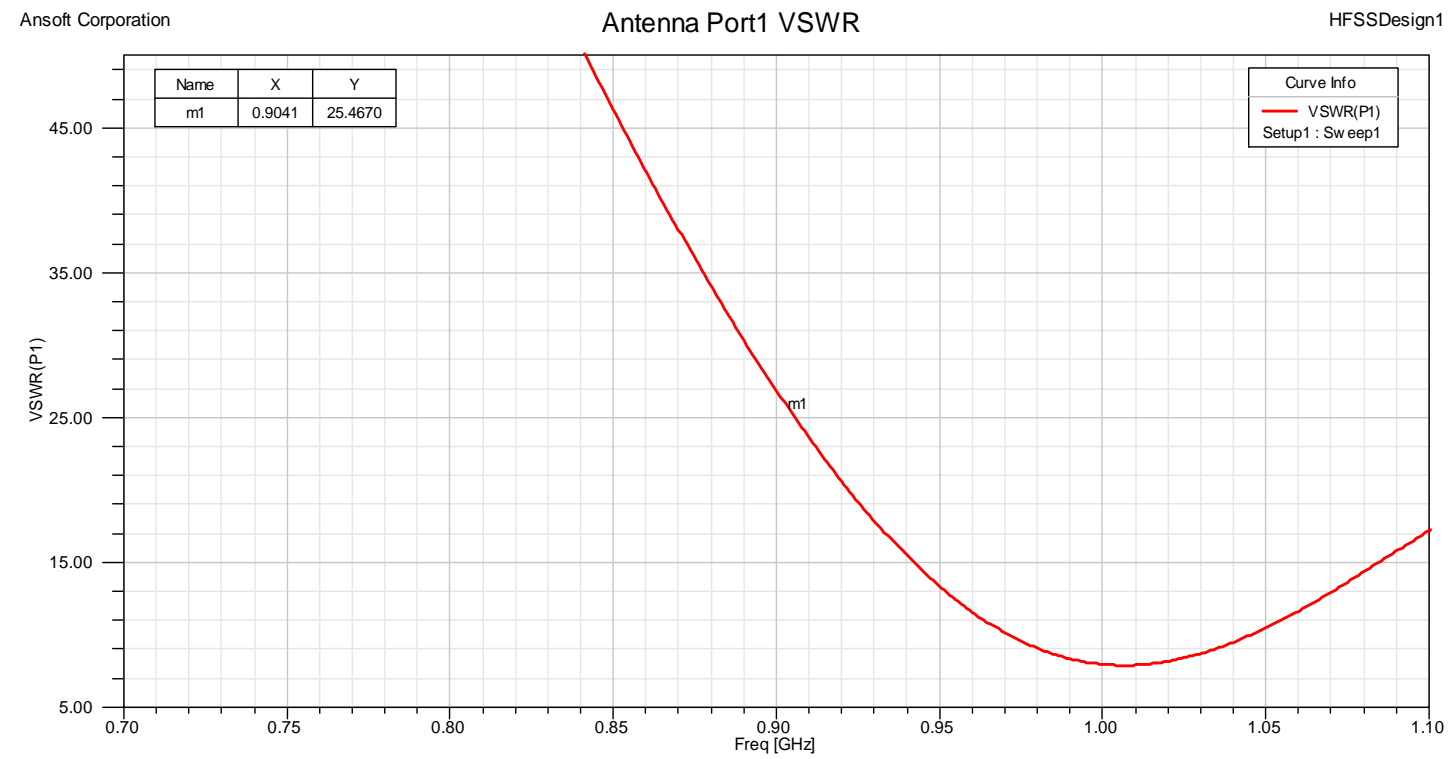

Figure 3.5.8: Short meandering-monopole input-port Voltage Standing Wave Ratio (VSWR) before addition of a matching network.

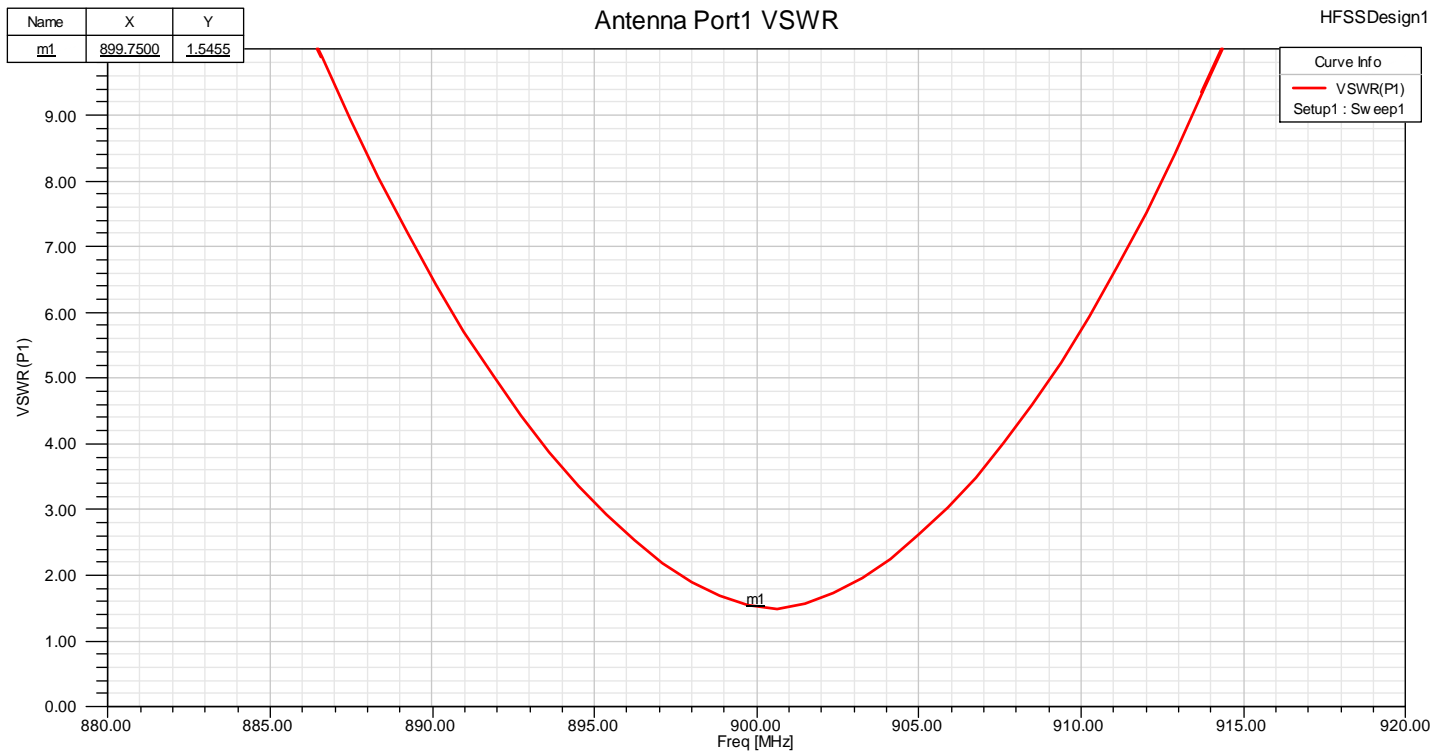

Figure 3.5.9: Short meandering-monopole input-port Voltage Standing Wave Ratio (VSWR) after addition of a matching network. 


\subsection{Compact Meandering-Monopole Simulation}

\subsubsection{Antenna Orientation and Matching Circuit}

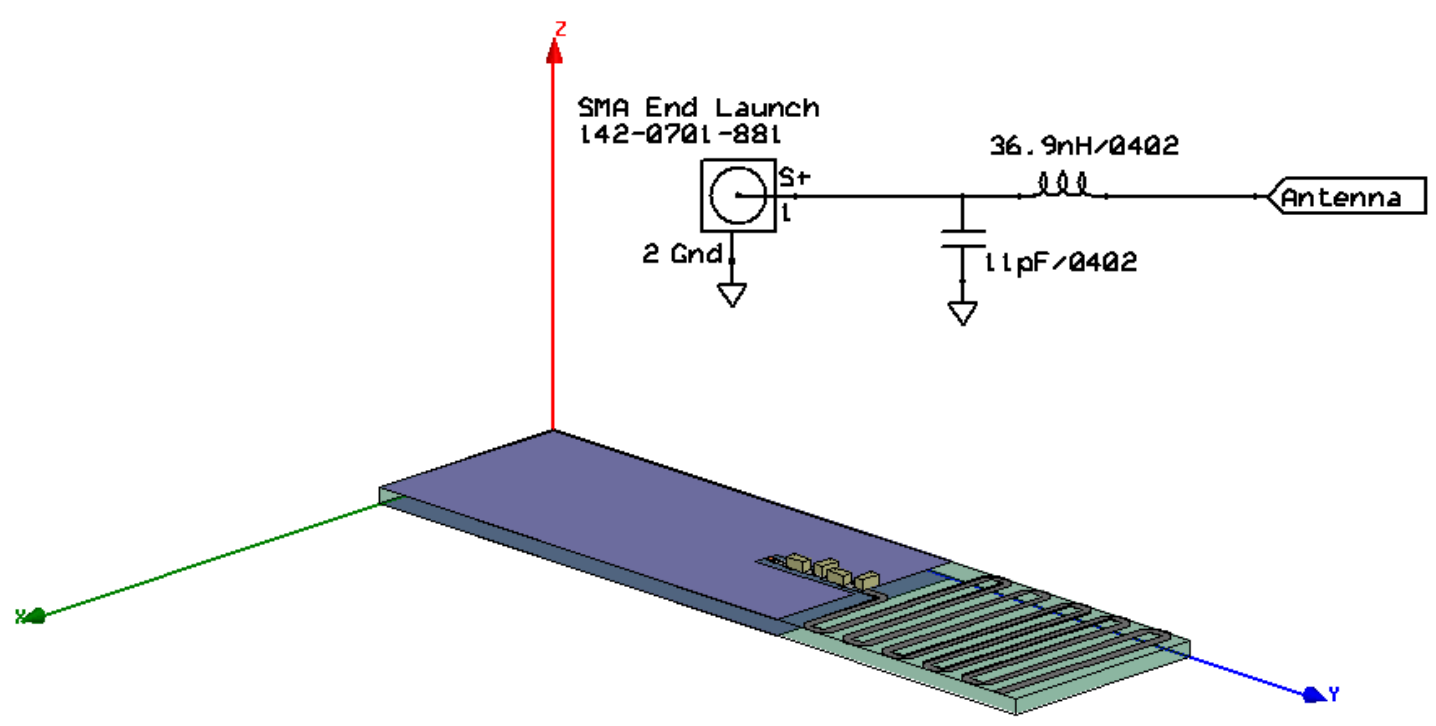

Figure 3.6.1: Compact meandering-monopole isometric orientation and simulated matching network required for $50-0 \mathrm{hm}$ match.

\subsection{Simulated 3D Radiation Pattern Gain (dB) after Matching}
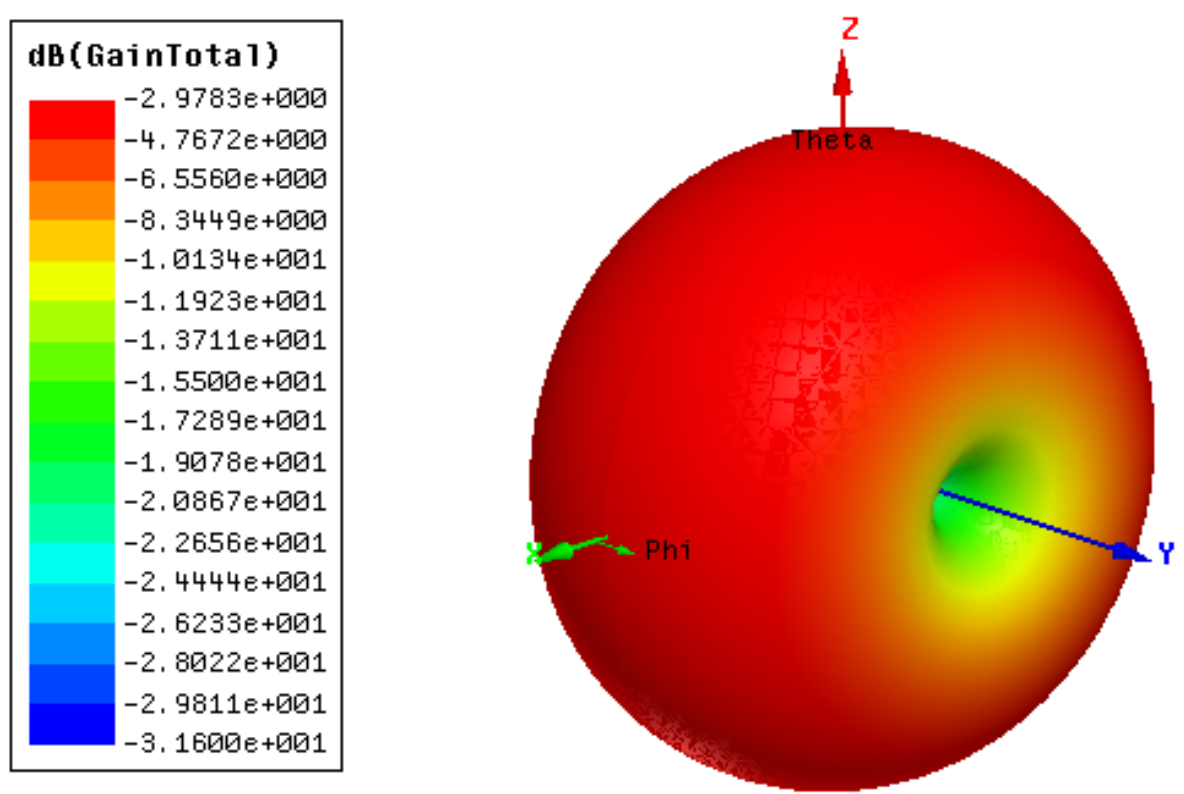

Figure 3.7.1: Compact meandering-monopole simulated 3D-radiation pattern expressed as Gain in $\mathrm{dB}$ for the isometric orientation before addition of a matching network. 


\subsubsection{Simulated 2D Radiation Pattern after Matching}
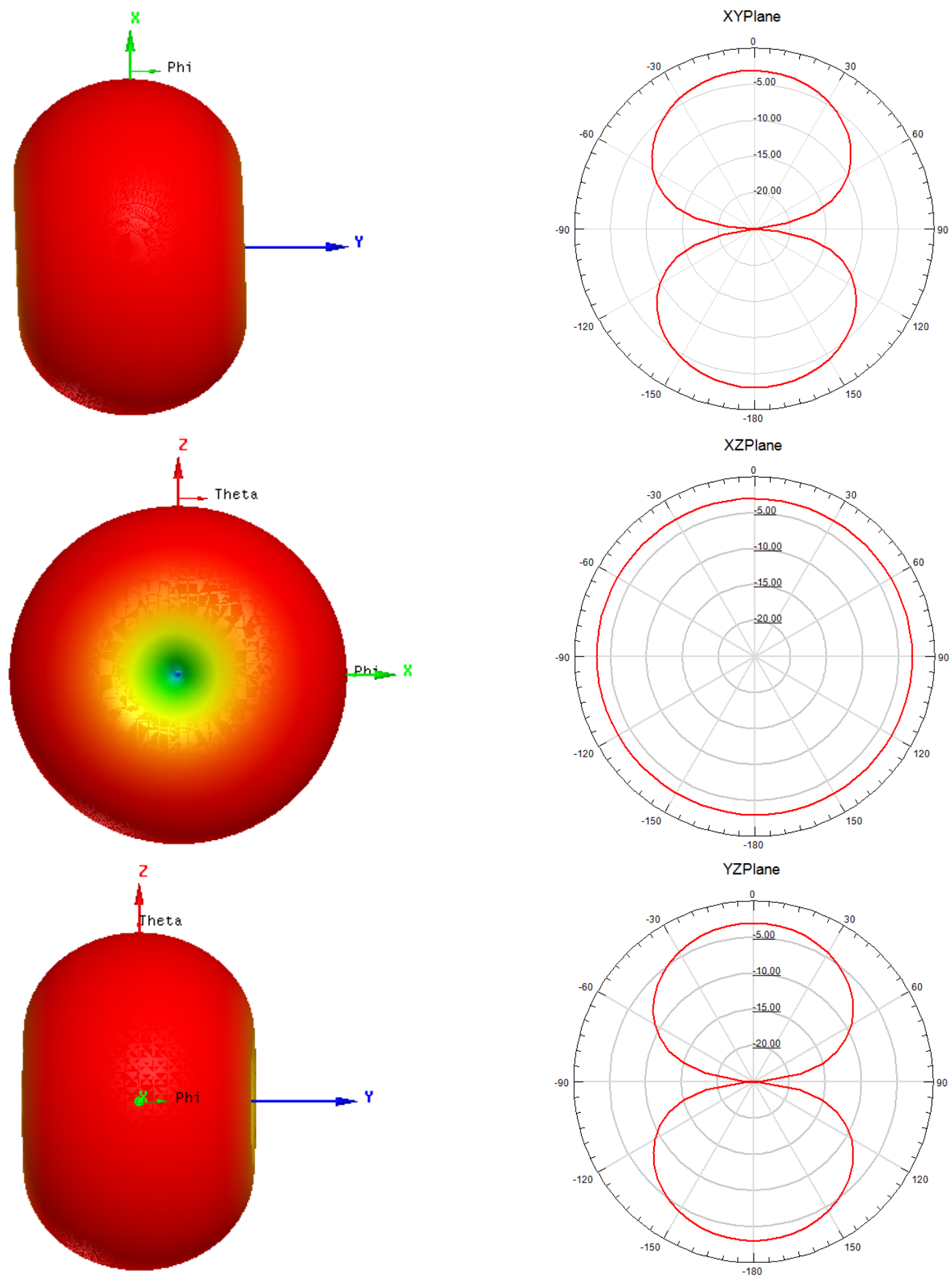

Figure 3.7.2: Compact meandering-monopole simulated 2D-radiation pattern expressed as Gain in $\mathrm{dB}$ for $\mathrm{XY}, \mathrm{XZ}$, and $\mathrm{YZ}$ planes before addition of a matching network. 


\subsubsection{Simulated S11 Reflection Coefficient (dB)}

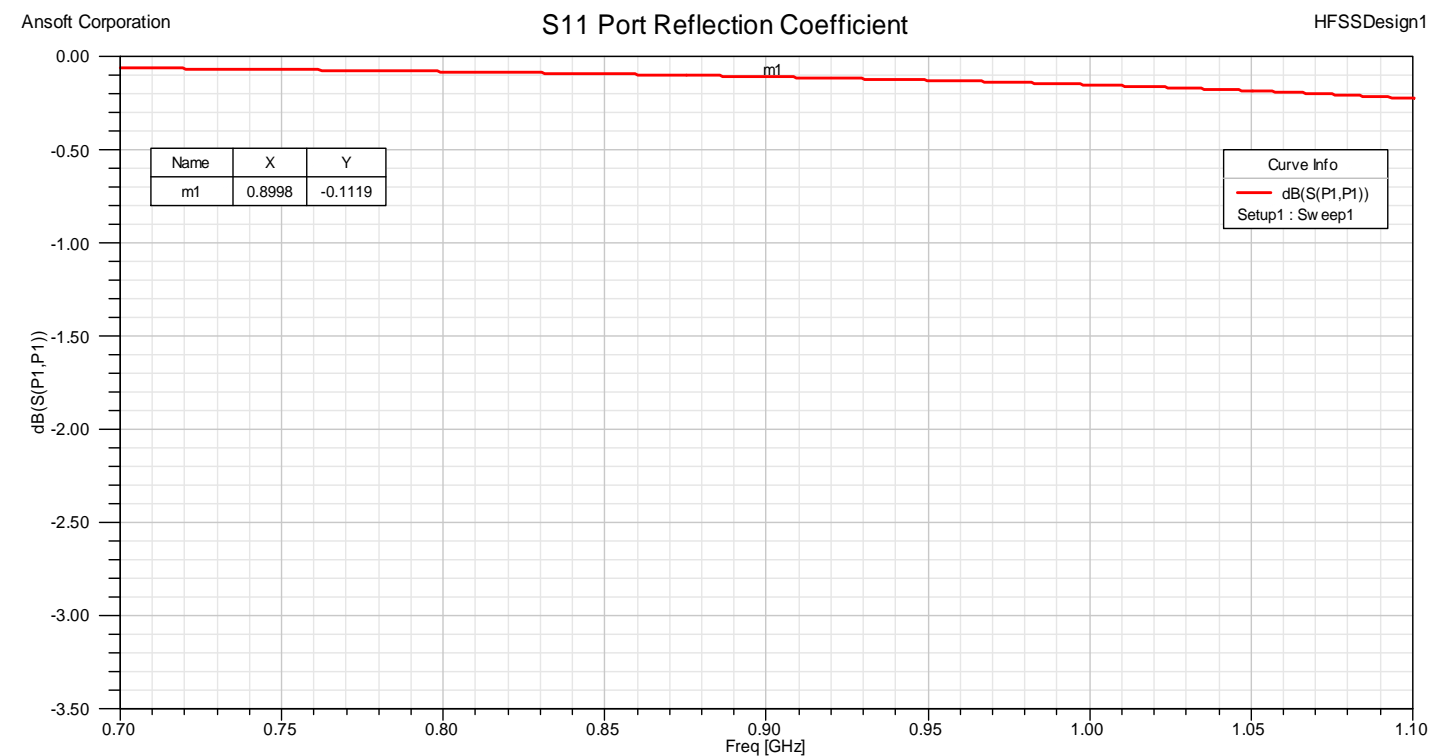

Figure 3.7.3: Compact meandering-monopole input-port reflection coefficient (S11) expressed in $\mathrm{dB}$ before addition of a matching network.

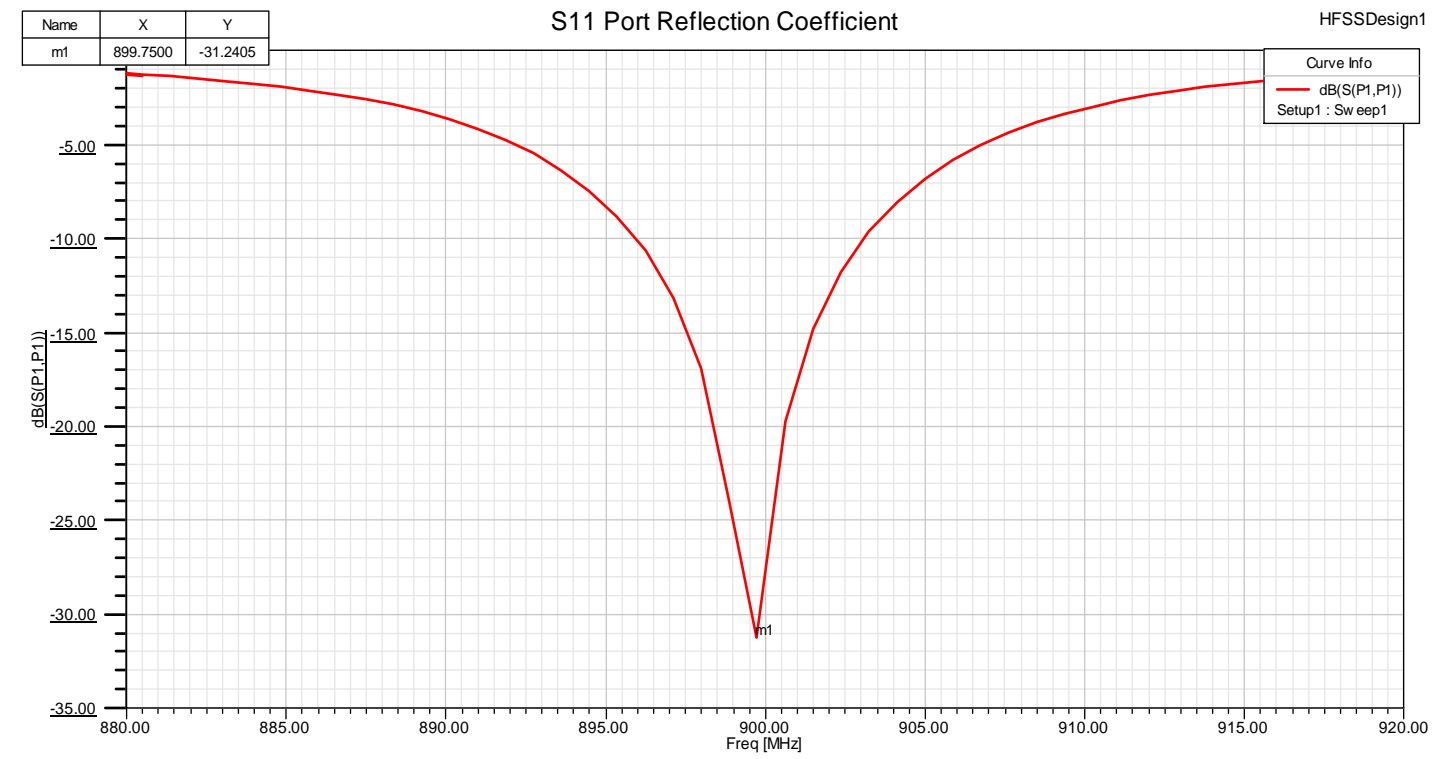

Figure 3.7.4: Compact meandering-monopole input-port reflection coefficient (S11) expressed in dB after addition of a matching network. 


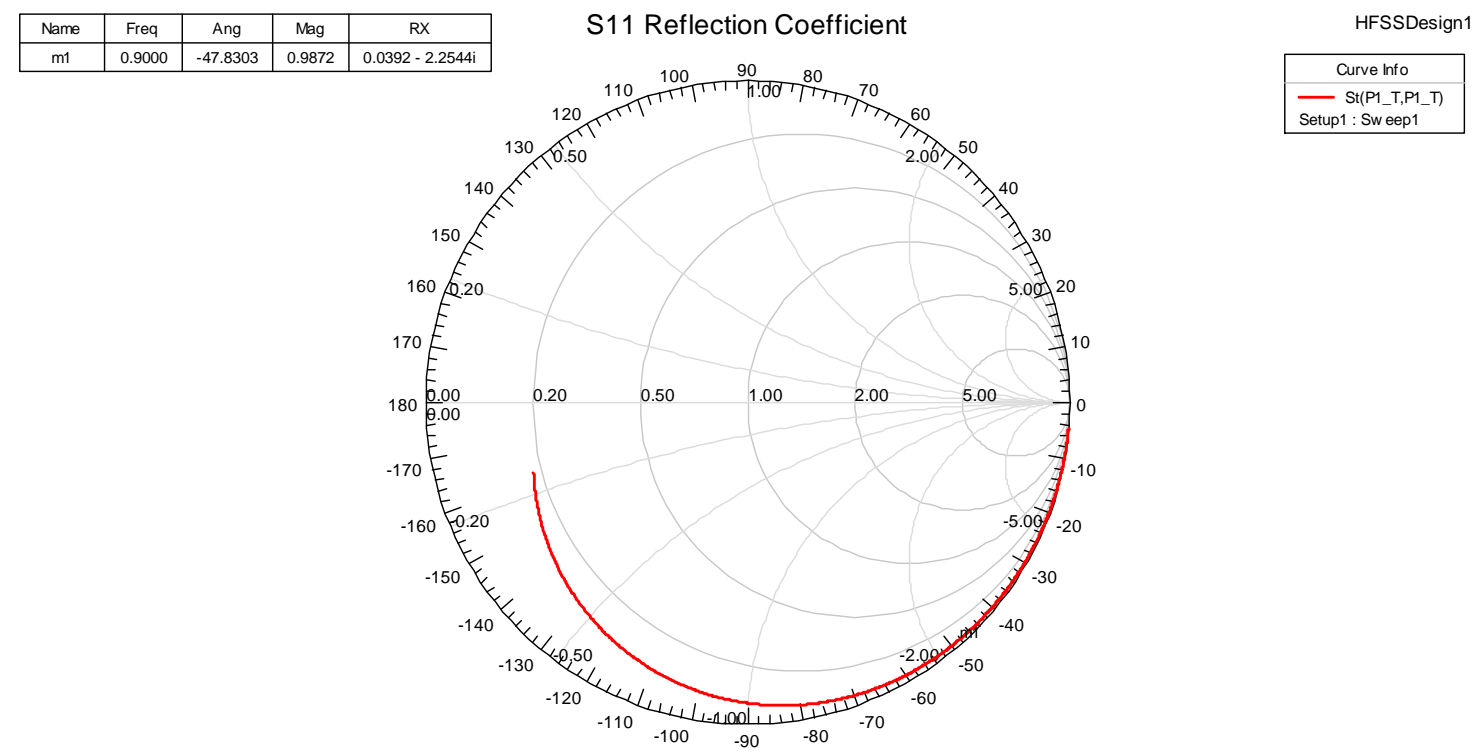

Figure 3.7.5: Compact meandering-monopole input-port reflection coefficient (Smith Chart) before addition of a matching network.

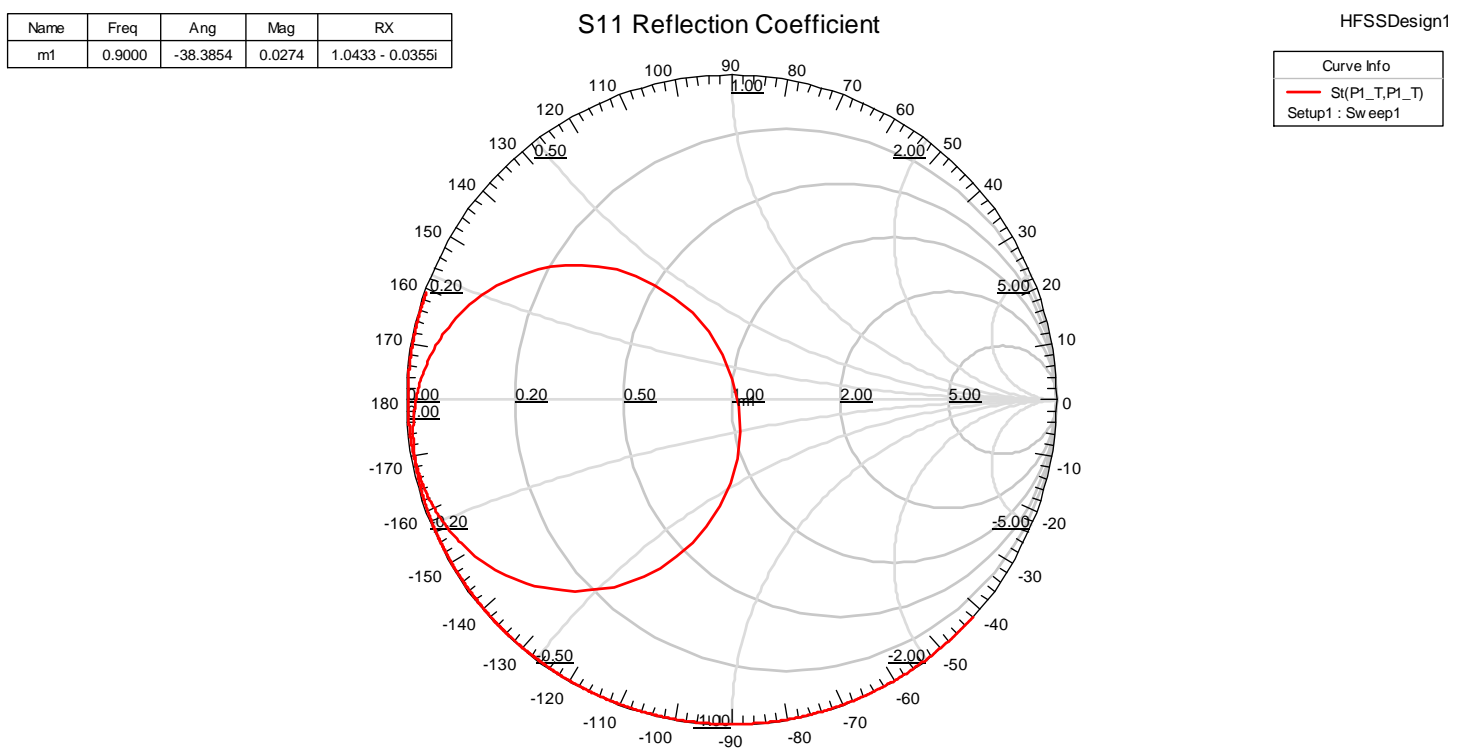

Figure 3.7.6: Compact meandering-monopole input-port reflection coefficient (Smith Chart) after addition of a matching network. 


\subsubsection{Simulated Voltage Standing Wave Ratio (VSWR)}

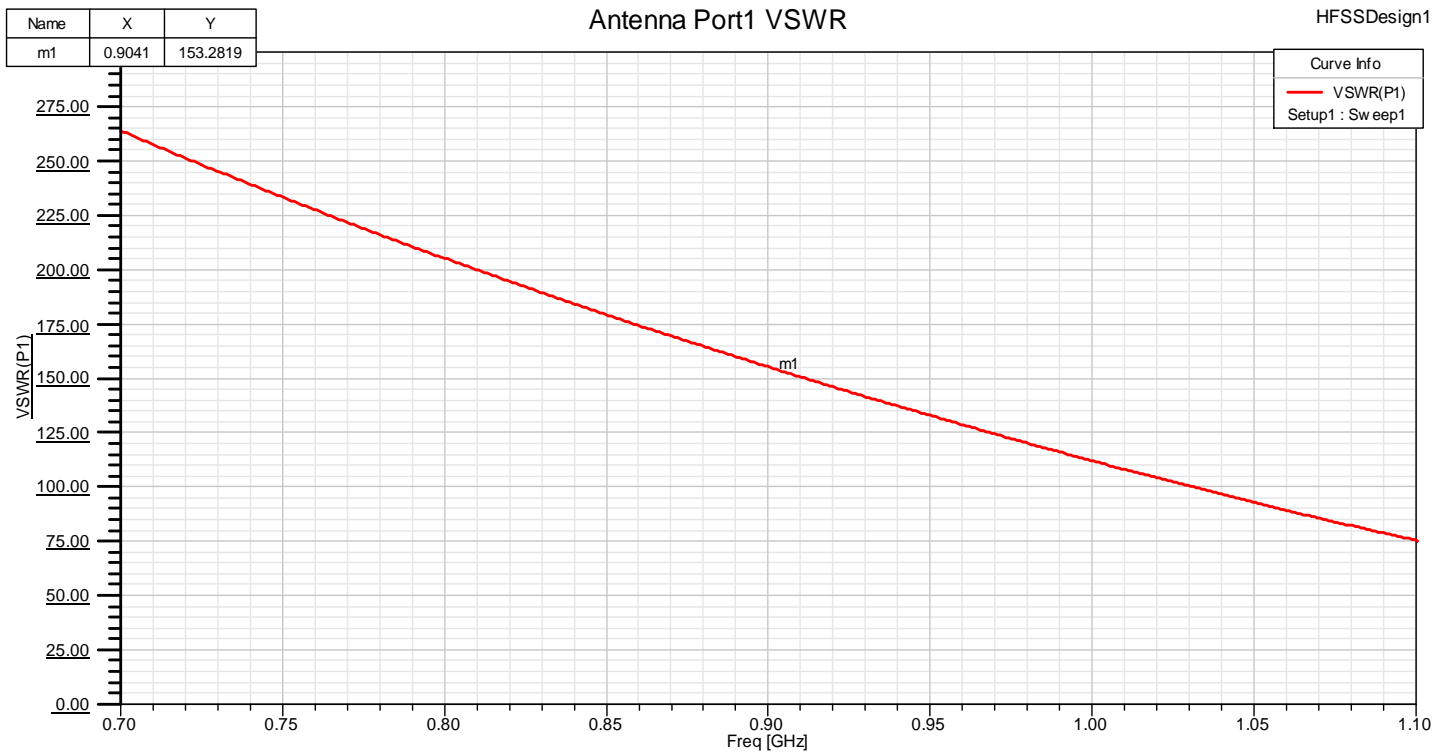

Figure 3.7.7: Compact meandering-monopole input-port Voltage Standing Wave Ratio (VSWR) before addition of a matching network.

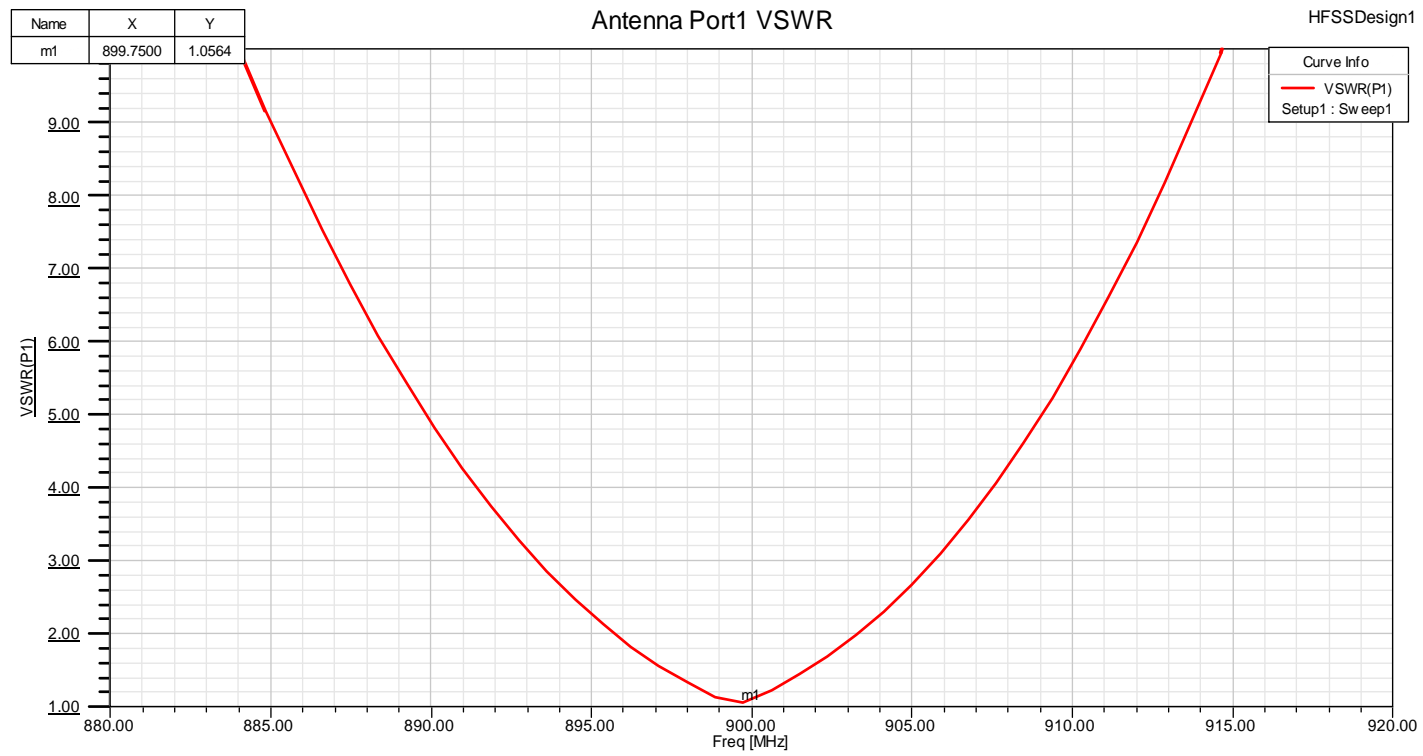

Figure 3.7.8: Compact meandering-monopole input-port Voltage Standing Wave Ratio (VSWR) after addition of a matching network. 


\subsection{Loop Antenna Simulation}

\subsubsection{Antenna Orientation and Matching Circuit}

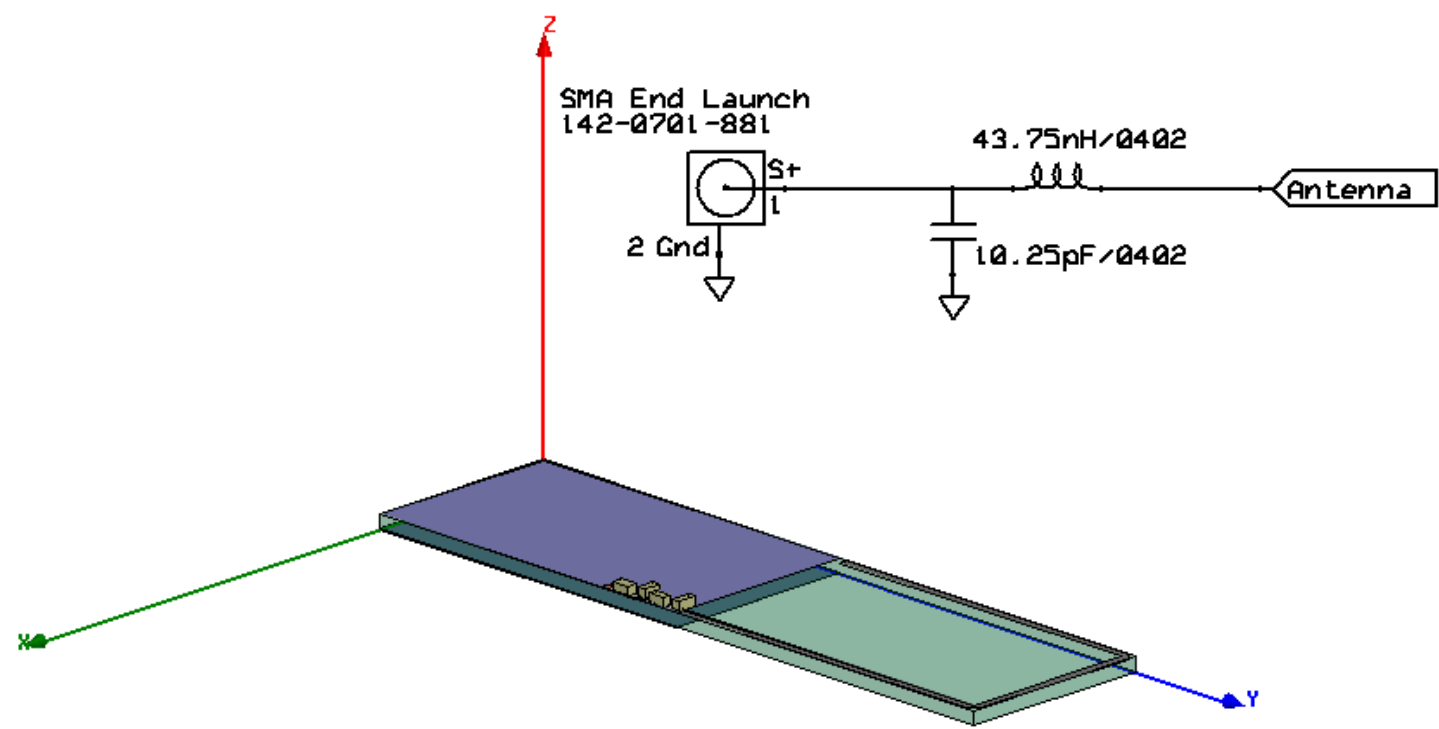

Figure 3.8.1: Loop antenna isometric orientation and simulated matching network required for 50-Ohm match.

\subsubsection{Simulated 3D Radiation Pattern Gain (dB) after Matching}
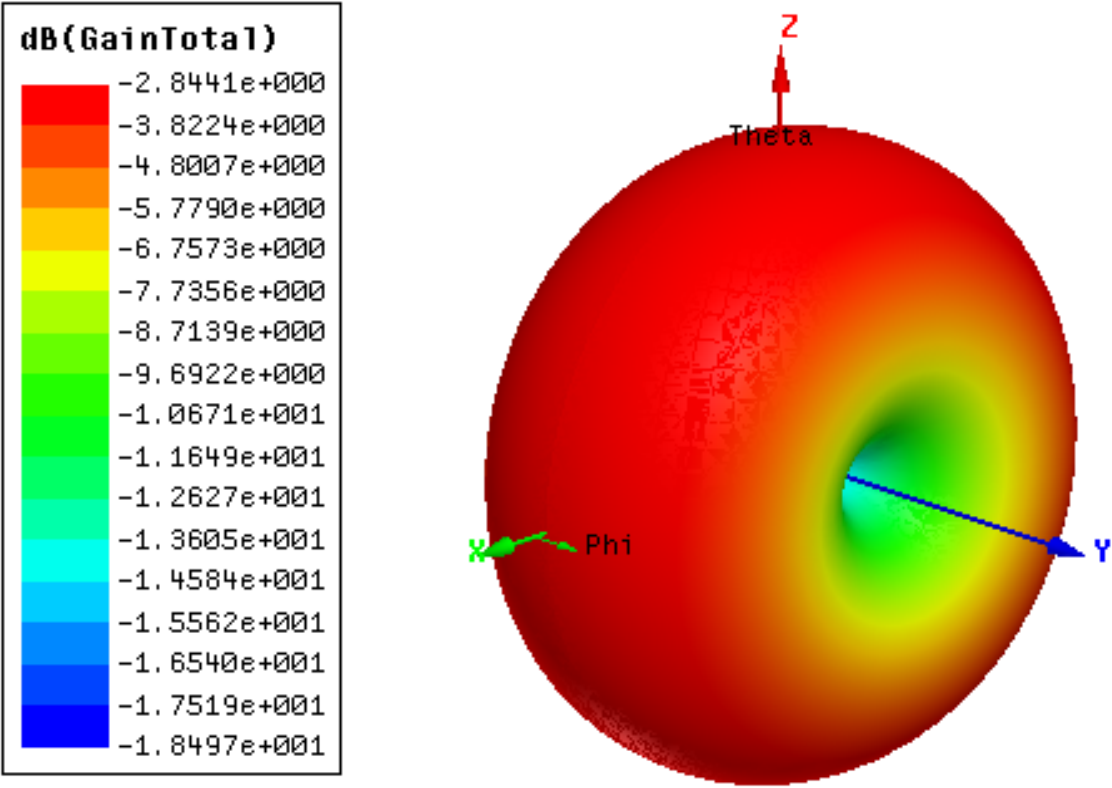

Figure 3.8.2: Loop antenna simulated 3D-radiation pattern expressed as gain in $\mathrm{dB}$ for the isometric orientation before addition of a matching network. 


\subsubsection{Simulated 2D Radiation Pattern after Matching}
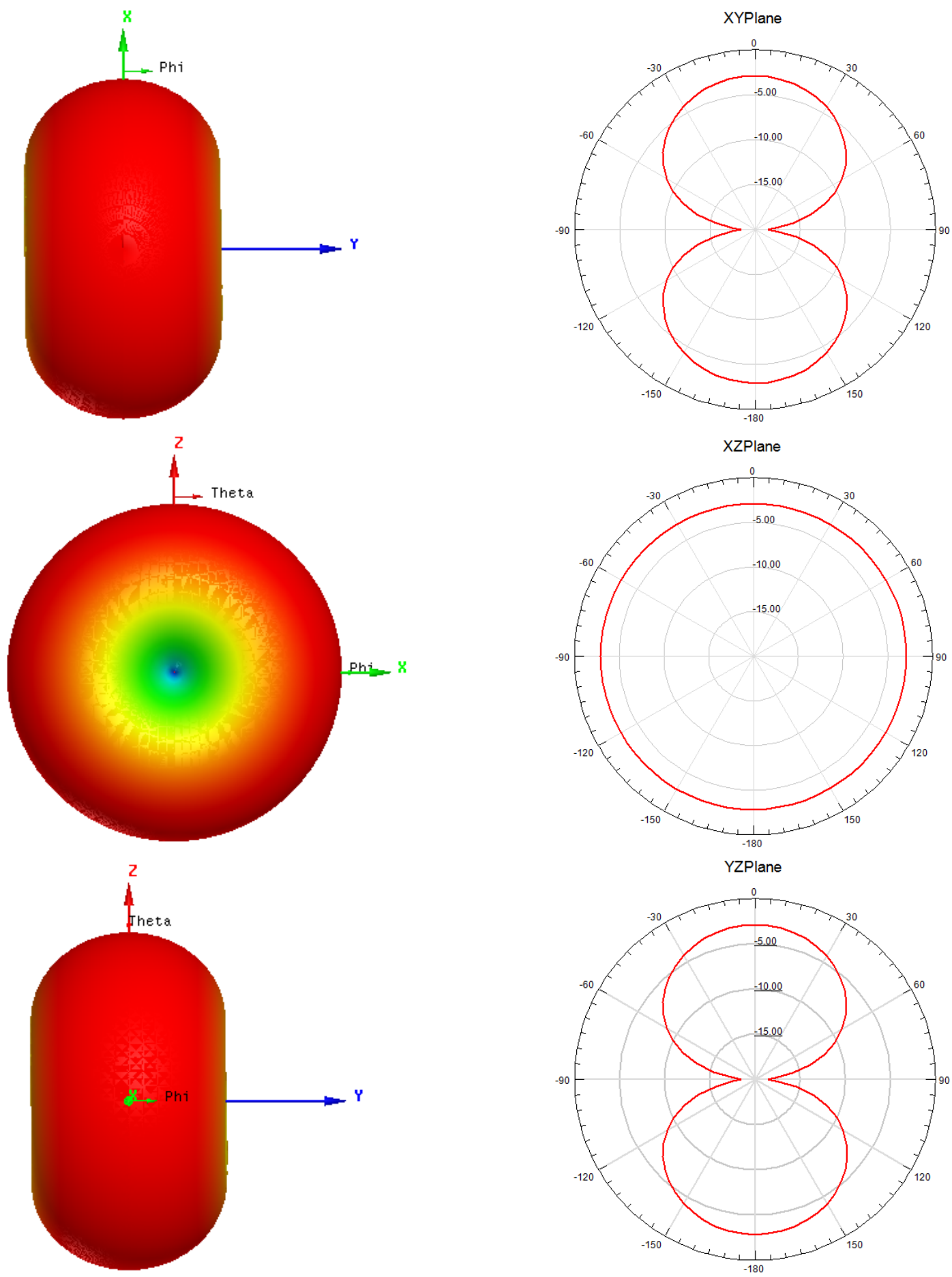

Figure 3.8.3: Loop antenna simulated 2D-radiation pattern expressed as gain in $\mathrm{dB}$ for $\mathrm{XY}, \mathrm{XZ}$, and YZ planes before addition of a matching network. 


\subsubsection{Simulated S11 Reflection Coefficient (dB)}

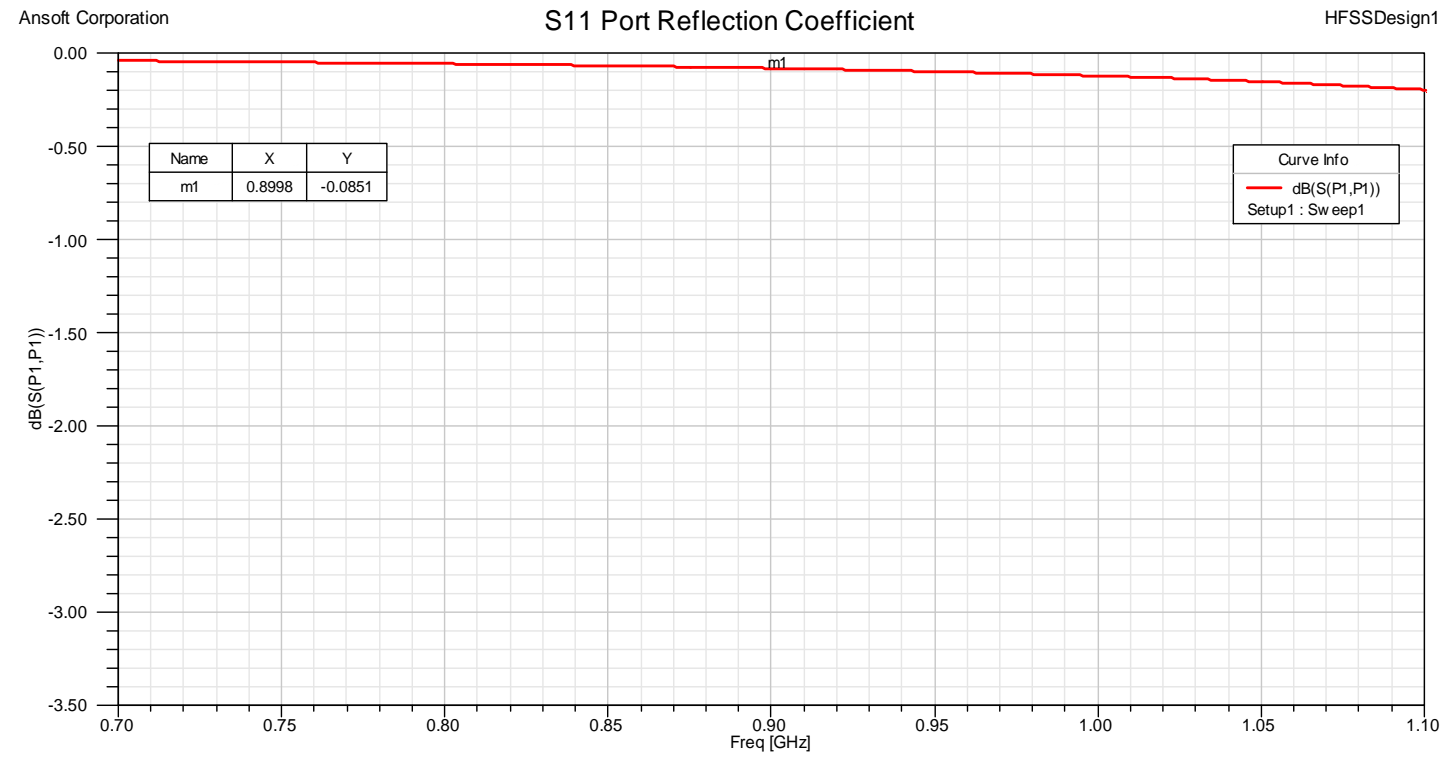

Figure 3.8.4: Loop antenna input-port reflection coefficient (S11) expressed in dB before addition of a matching network.

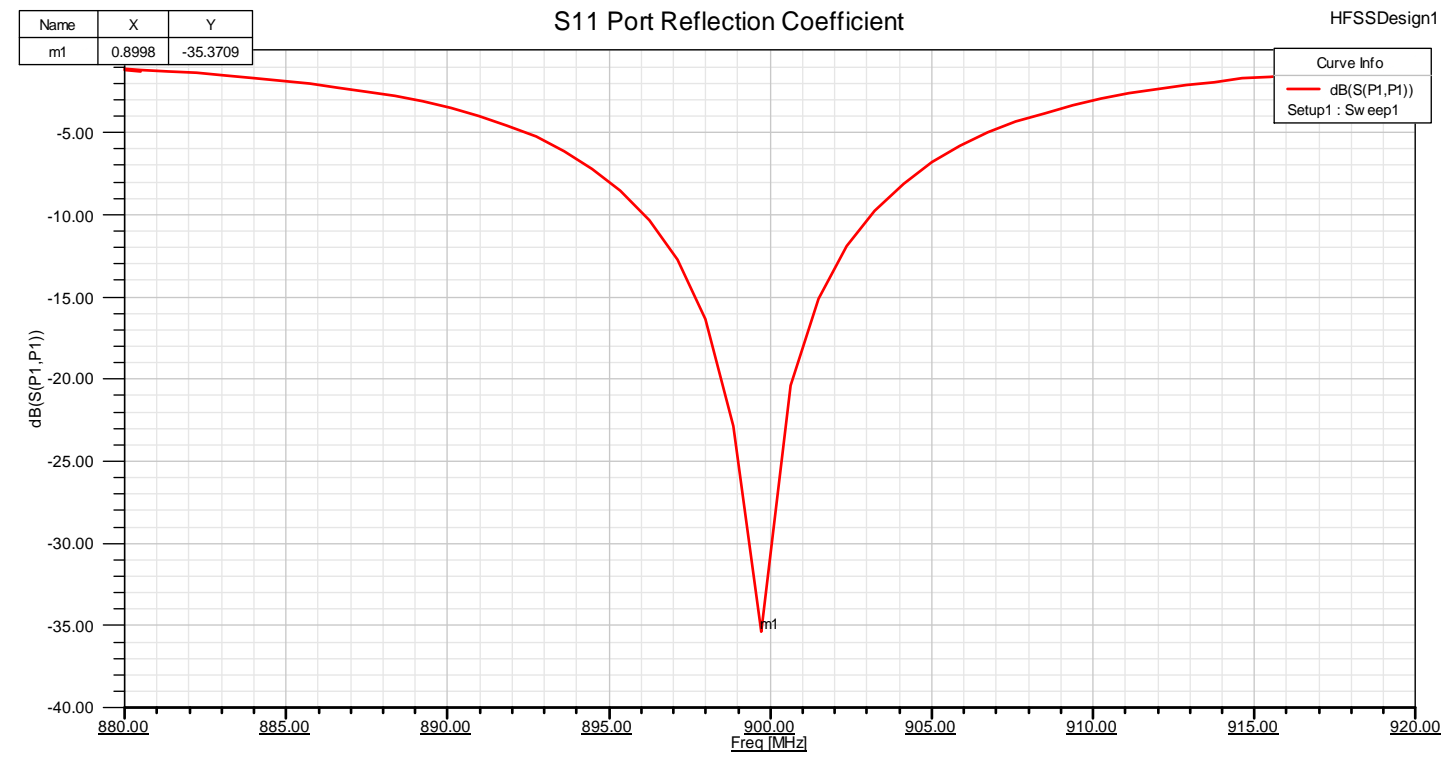

Figure 3.8.5: Loop antenna input-port reflection coefficient (S11) expressed in dB after addition of a matching network. 


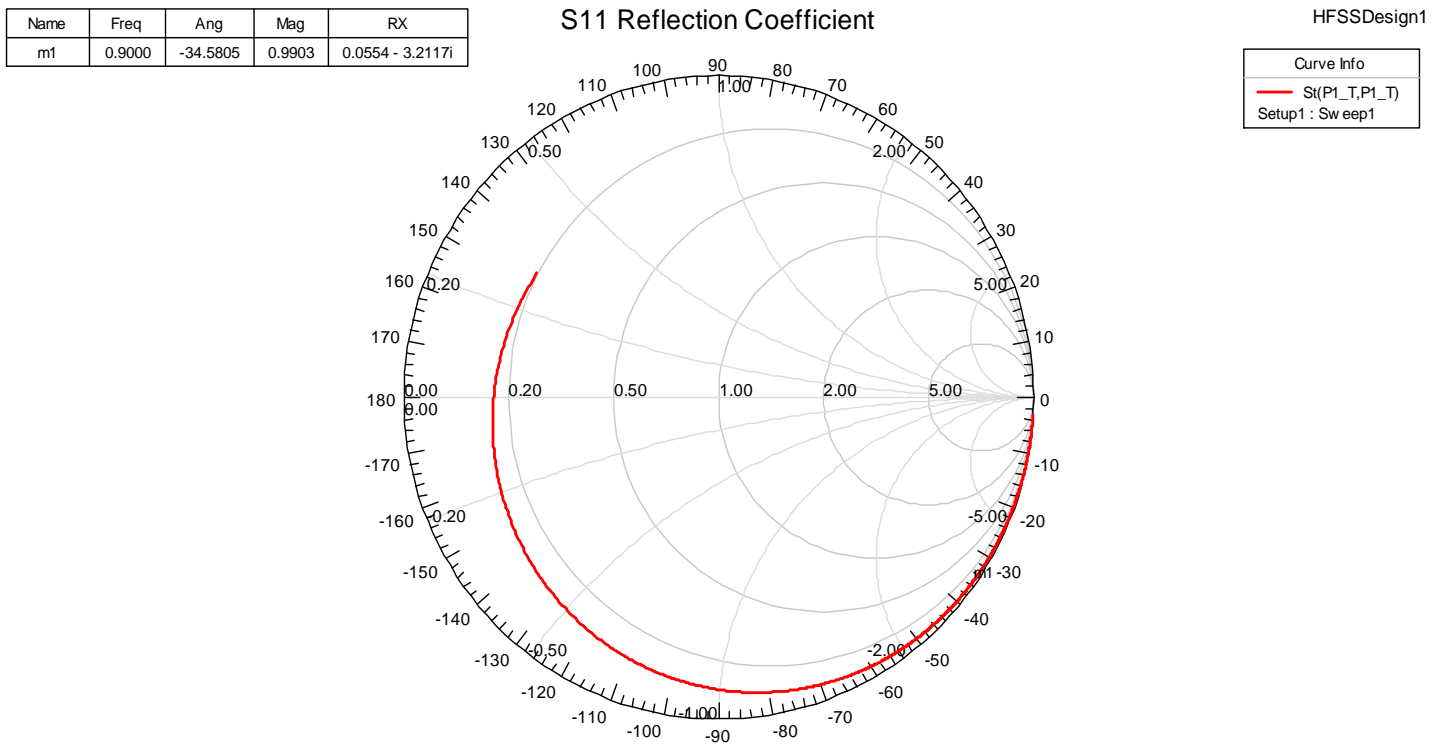

Figure 3.8.6: Loop antenna input-port reflection coefficient (Smith Chart) before addition of a matching network.
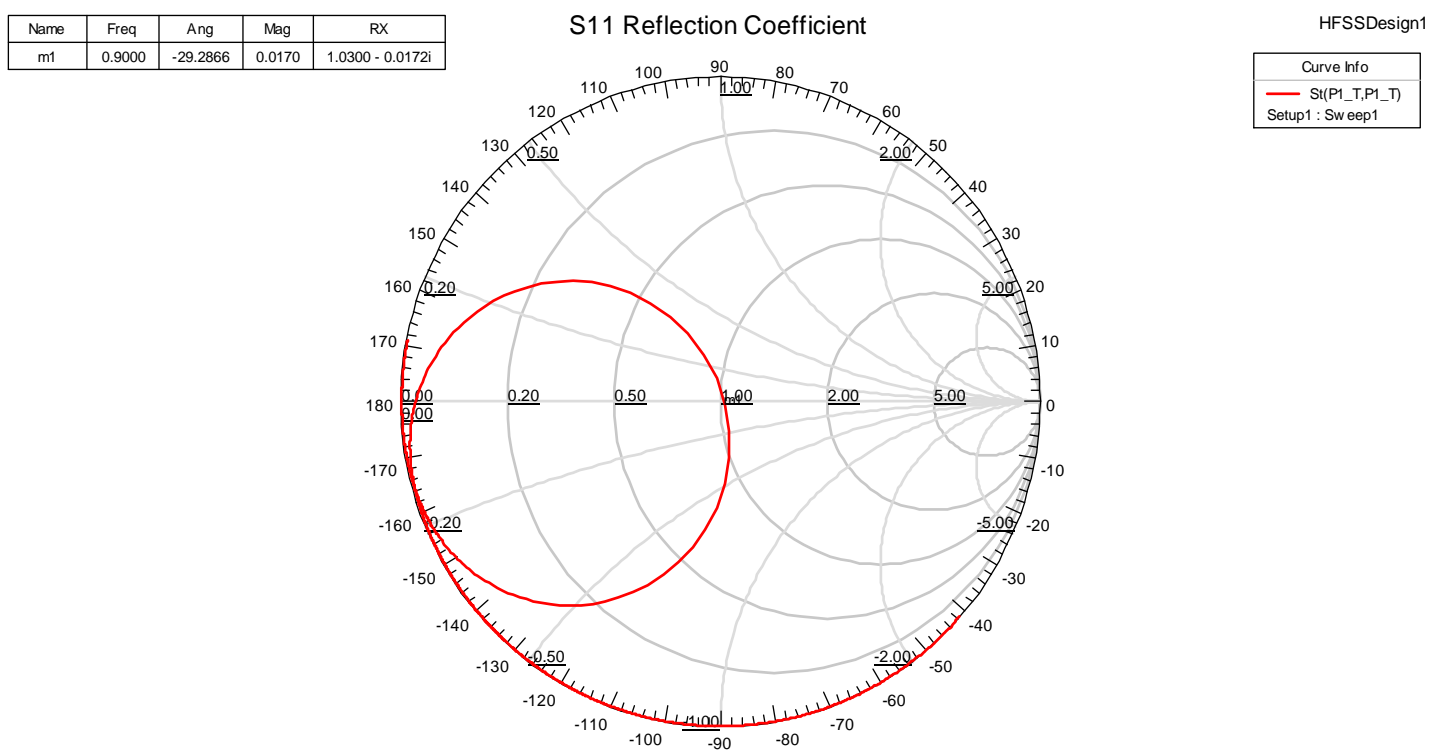

Figure 3.8.7: Loop antenna input-port reflection coefficient (Smith Chart) after addition of a matching network. 


\subsubsection{Simulated Voltage Standing Wave Ratio (VSWR)}

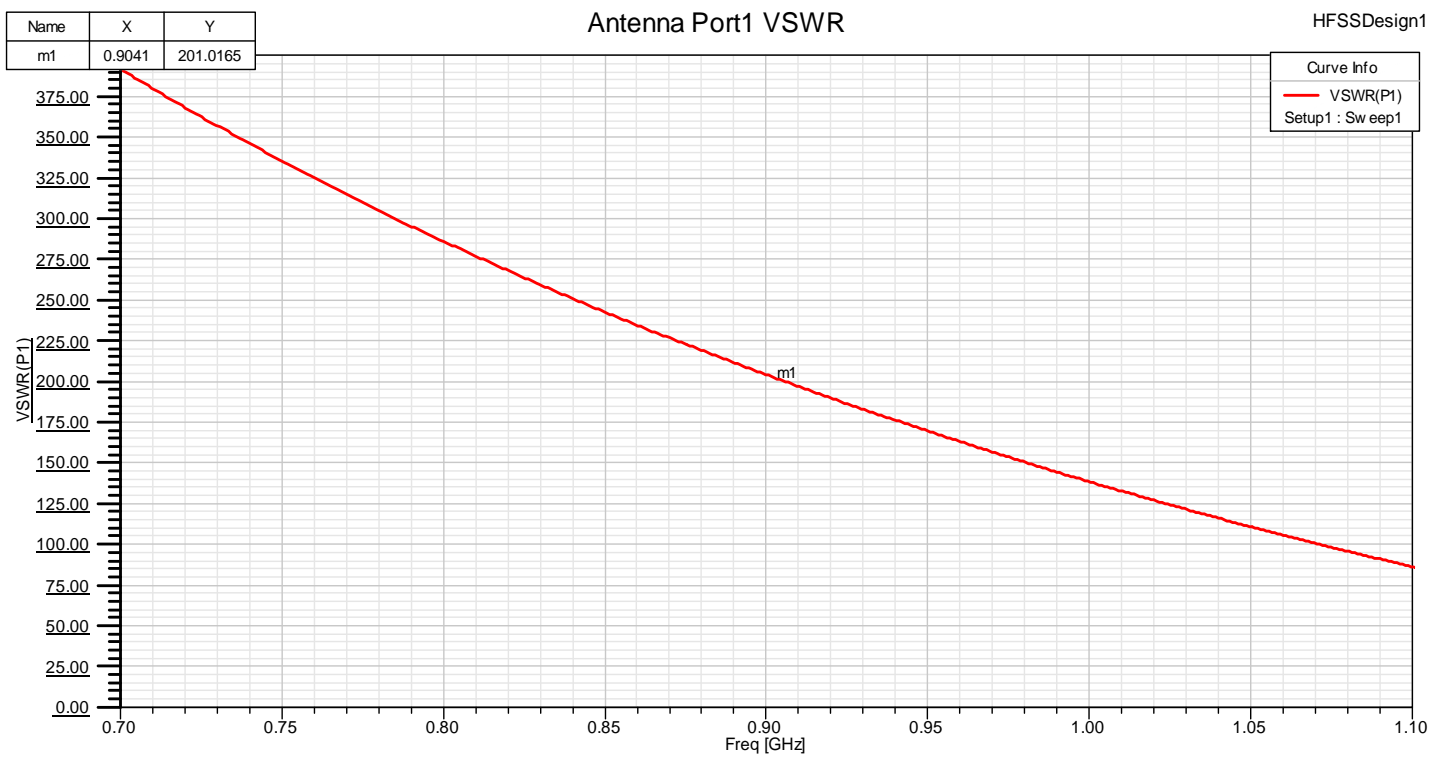

Figure 3.8.8: Loop antenna input-port Voltage Standing Wave Ratio (VSWR) before addition of a matching network.

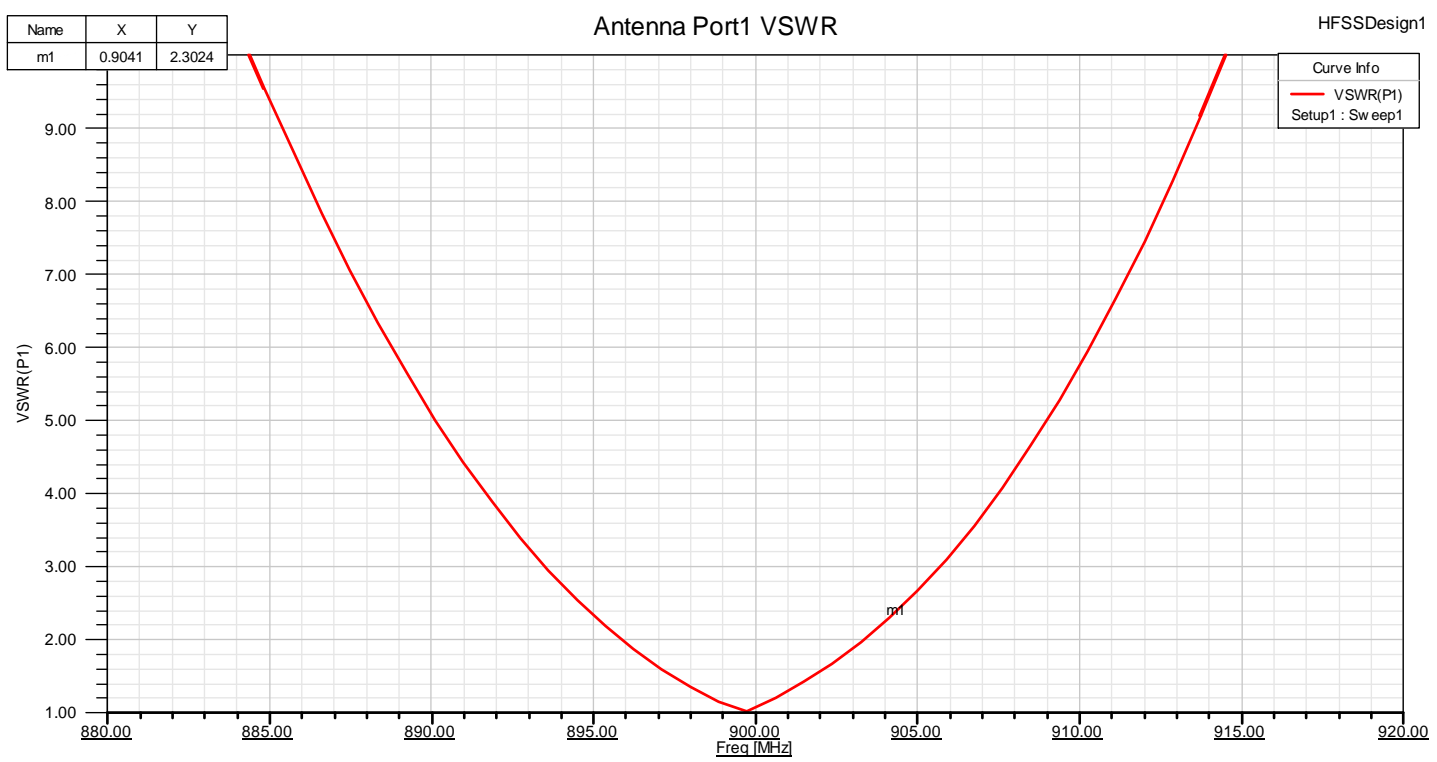

Figure 3.8.9: Loop antenna input-port Voltage Standing Wave Ratio (VSWR) after addition of a matching network. 


\subsection{Ansoft Simulation Results}

Based upon simulated data, an estimate of antenna performance is predicted before implementation of each design. Because the radiating element length of each antenna is arbitrarily based upon its PCB dimensions, the initial resonant frequency of each design is different. The simulated data predicts initial antenna behavior before addition of matching circuitry. This performance data is used to tune the antenna by adding lumped RLC boundary conditions to elements in the simulation model. Simulated data has shown each antenna has the ability to be matched and will resonate properly at $900 \mathrm{MHz}$. One can see the simulated gain is much lower than what would be normally expected for each printed antenna design. The behavior and simulation results of each design are summarized in the following paragraphs.

For the inverted-F antenna, simulation data predicts this antenna to have an initial resonant frequency of $895 \mathrm{MHz}$ and to be easily matched with a 10-nH series inductor and a 2.65-pF shunt capacitor. The radiating element length is intentionally longer to allow trimming of prototyped antennas for an exact match. The antenna bandwidth at 2:1 VSWR is expected to be $10.5 \mathrm{MHz}$ and should have a non uniform radiation pattern containing two lobes. It is expected to have an $\mathrm{S} 11$ reflection coefficient of $-24.65 \mathrm{~dB}$ with peak gain of $-13.9 \mathrm{dBi}$. The simulated gain is much lower than expected for this design.

For the long meandering monopole antenna, simulation data predicts this antenna to have an initial resonant frequency of $860 \mathrm{Mhz}$. The matching network is expected to contain a $28-\mathrm{nH}$ series inductor and 1.1-pF shunt capacitor. The antennas bandwidth at 2:1 VSWR is expected to be $10 \mathrm{MHz}$ and have a uniform monopoleshaped radiation pattern. It is expected to have an $\mathrm{S} 11$ reflection coefficient of -16.8 $\mathrm{dB}$ with peak gain of $-13.8 \mathrm{dBi}$. The simulated gain is much lower than expected for this design. 
For the short meandering monopole antenna, simulation data predicts the antenna to have an initial resonant frequency of $1.1 \mathrm{GHz}$. The matching network is expected to contain an $8.25-\mathrm{nH}$ series inductor with a $10-\mathrm{pF}$ shunt capacitor. The antennas bandwidth at 2:1 VSWR is expected to be $5.0 \mathrm{Mhz}$ and have a uniform monopole-shaped radiation pattern. It is expected to have an S11 reflection coefficient of $-14 \mathrm{~dB}$ and peak gain of $-11.2 \mathrm{dBi}$. The simulated gain is much lower than expected for this design.

For the compact meandering monopole antenna, simulation data predicts the antenna to have an initial resonant frequency much larger than the 1.1-GHz upper simulation frequency limit. The matching is expected to contain a $36.9-\mathrm{nH}$ series inductor and 11-pF shunt capacitor. The antenna bandwidth at 2:1 VSWR is expected to be $7 \mathrm{MHz}$ and have a uniform monopole-shaped radiation pattern. It is expected to have an $\mathrm{S} 11$ reflection coefficient of $-31 \mathrm{~dB}$ with peak gain of $-2.98 \mathrm{dBi}$. This simulated gain is better than other designs but is still much lower than expected for this antenna.

For the loop antenna, simulation data predicts the antenna to have an nitial resonant frequency much larger than the 1.1-GHz upper simulation frequency limit. The matching network is expected to contain a $43.75-\mathrm{nH}$ series inductor and $10.25-\mathrm{pF}$ shunt capacitor. The antenna bandwidth at 2:1 VSWR is expected to be $7.0 \mathrm{MHz}$ and have a uniform monopole-shaped radiation pattern. It is expected to have an S11 reflection coefficient of $-35 \mathrm{~dB}$ with gain of $-2.84 \mathrm{~dB}$. This simulated gain is better than other designs but is still much lower than expected for this antenna.

These simulated results are compared with actual prototyped antenna measurements in the results Chapter 7 of the report. 


\section{Prototype and Test Capabilities}

\subsection{Antenna Prototyping}

This section introduces the antenna prototyping process and equipment needed for antenna fabrication. Each antenna is drafted using Microstation-V8 CAD software. Completed drawings are exported in Gerber file format (as shown below in Figure 4.1.1), containing information about each layer of the PCB. The file is then post processed to remove unwanted layers and then panelized to completely fill an entire sheet. Rapid prototyping equipment made by LPKF Laser and Electronics is then used to route copper-clad laminate into working antenna prototypes. A single 9x13 inch sheet has 10 panels and is capable of producing 50 total antennas.

(a)

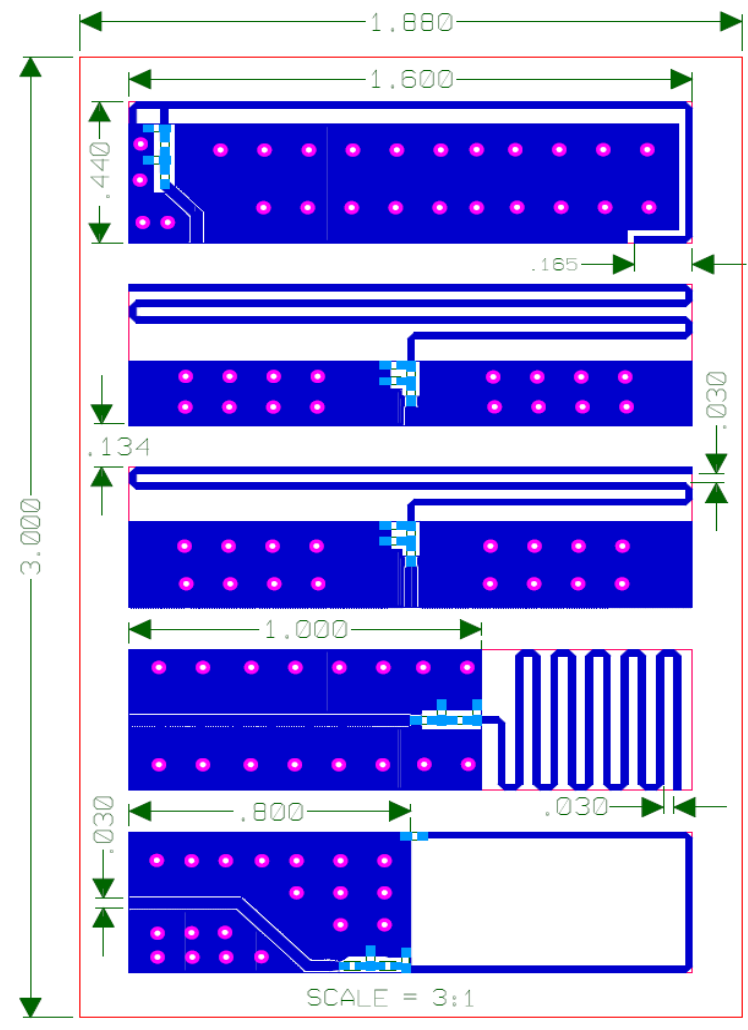

Figure 4.1.1: Microstation Gerber file, from top-to-bottom: (a) Inverted-F antenna, (b) Long meandering-monopole antenna, (c) Short meandering-monopole antenna, (d) Compact meandering-monopole antenna, and (e) Loop antenna. 
The fabrication process begins when a sheet of blank copper-cladded FR4 material is placed in the automated router. Initially, only plated through-hole vias are drilled in the base material before it is transferred to an LPKF Minicontac-RS electroplating system, shown below in Figure 4.1.2. This system uses four chemical baths where the samples are cleaned, degreased, pre-treated with activator, and then copper plated in a final galvanic bath. This system uses reverse-pulse plating to evenly grow copper material connecting all through-hole vias.

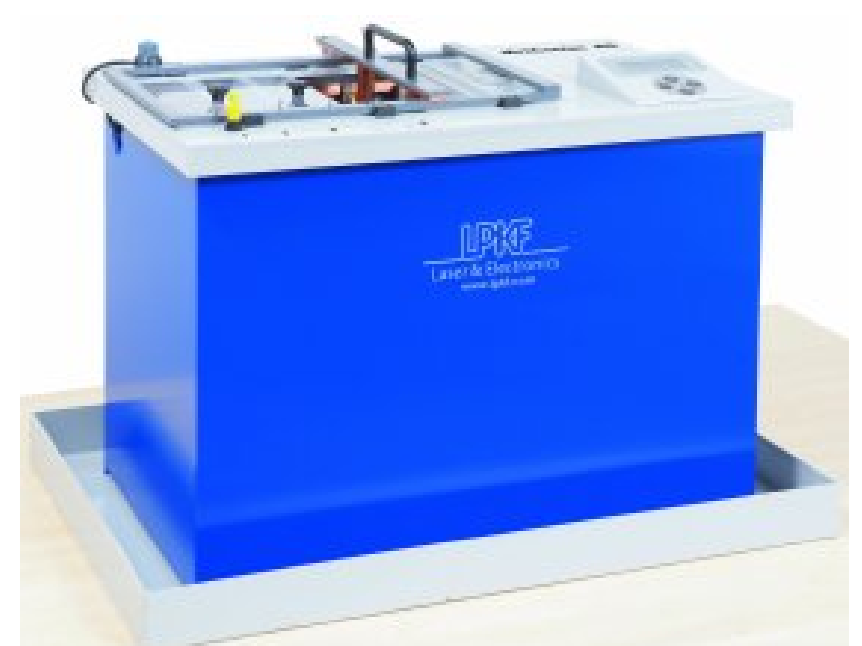

Figure 4.1.2: LPKF Minicontac-RS through-hole electroplating bath.

Once completed, the electroplated sample is cleaned and placed back in the automated router for final routing of top and bottom sides. The LPKF Automated Router and completed prototypes are shown in Figure 4.1.3 and Figure 4.1.4. There are striations visible in the completed prototypes where copper plating is not evenly deposited. This behavior occurs due to excessive removal of activator material in the cleaning process between activation and galvanic baths. Overall, the plating process works reasonably well and the affected antennas with uneven areas are not included in measurements. Four antennas from each design are matched for 50-Ohms and measured data is compared with simulated data in Chapter 7 results. 


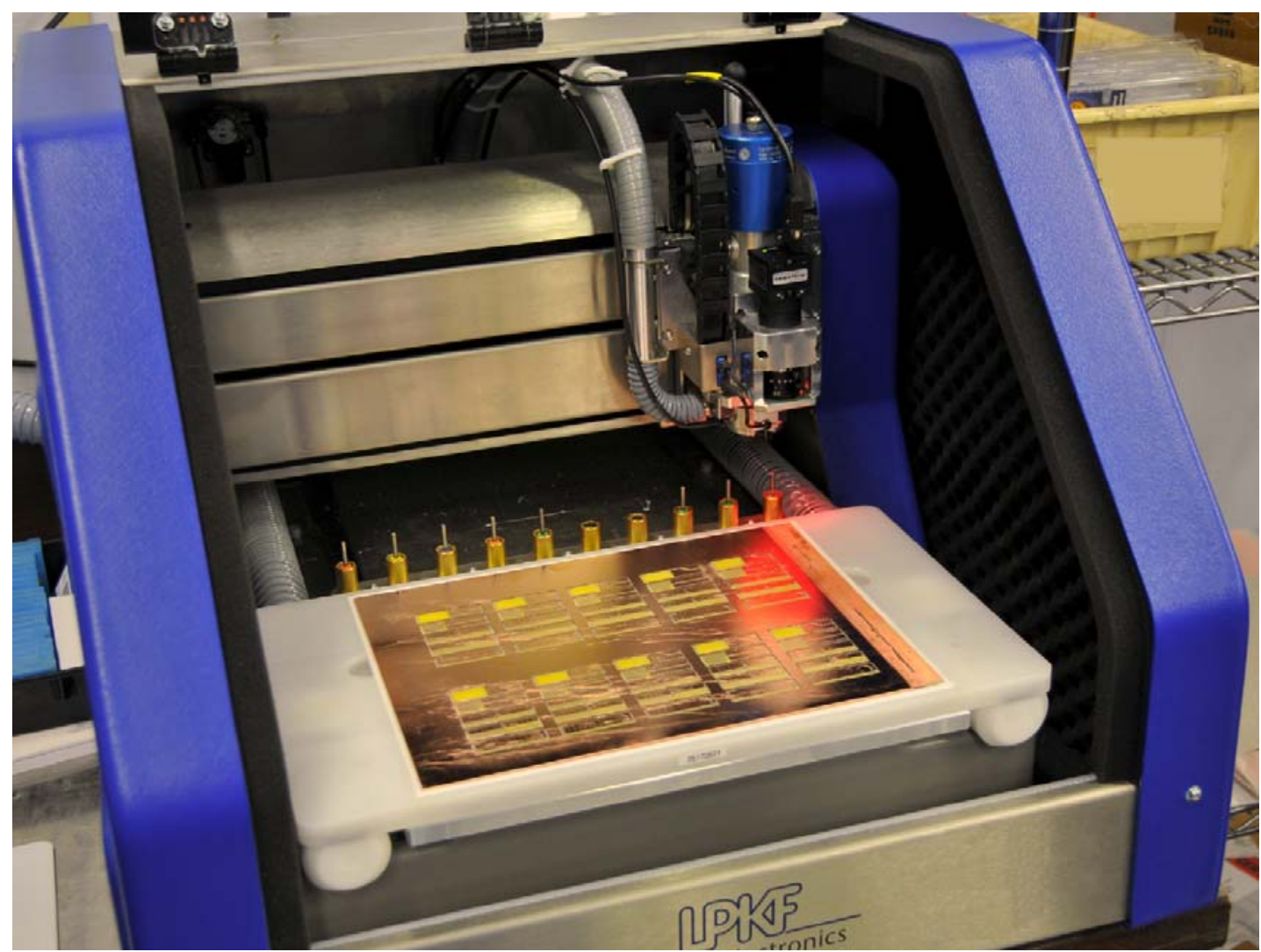

Figure 4.1.3: The LPKF automated protomat router and fabricated prototypes.

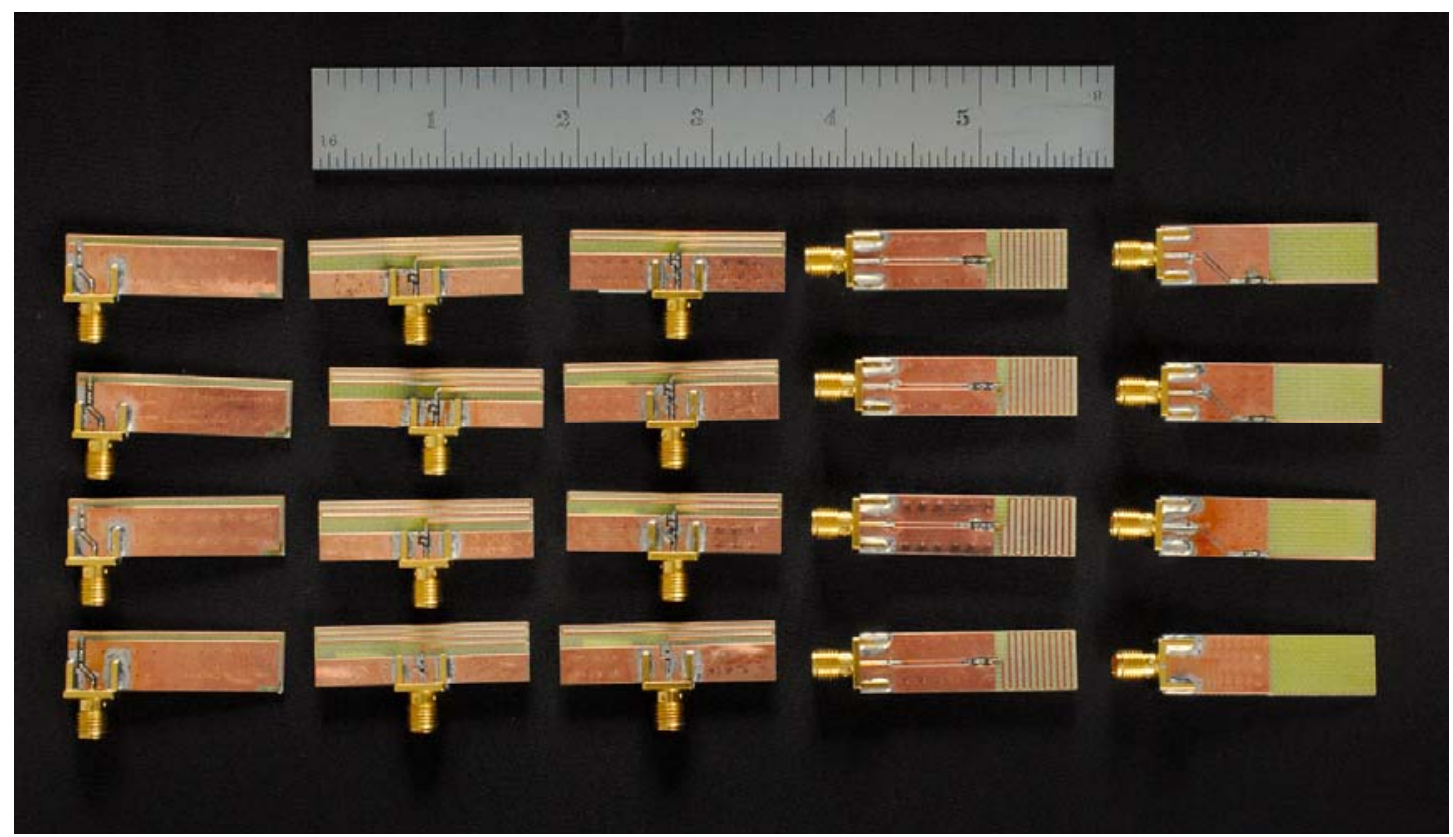

Figure 4.1.4: Completed antenna prototypes. 


\subsection{Antenna S-Parameter Measurement}

An Agilent N3383A Network Analyzer is used to measure antenna Sparameter reflection coefficients. A single port S11-measurement is used to determine how efficiently the antenna is fed and characterize matching network performance. A Lindgren shielded enclosure is used to help stabilize measurements by attenuating outside noise sources up to $70 \mathrm{~dB}$. Prototypes are placed inside the shielded enclosure for collection of magnitude, Smith chart, and VSWR data plots of the reflection coefficients that are presented in Chapter 5. A 22-inch circular mounting base is used as a large ideal ground plane inside the chamber. This improves radiator performance and provides a stable antenna mounting platform. Calibration of the network analyzer is performed at the antenna input port to compensate for all SMA, feed-through, and cable losses. Figure 4.2.1 and Figure 4.2.2 show the HP network analyzer and Lindgren shielded enclosure used for testing.

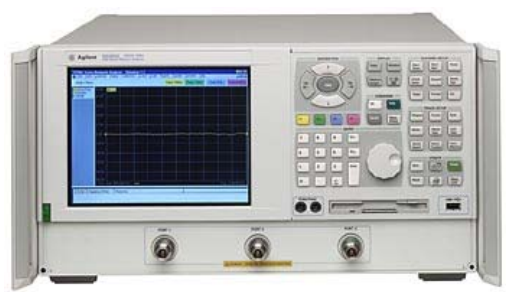

Figure 4.2.1: Agilent HP N3383A 300KHz-9GHz network analyzer.

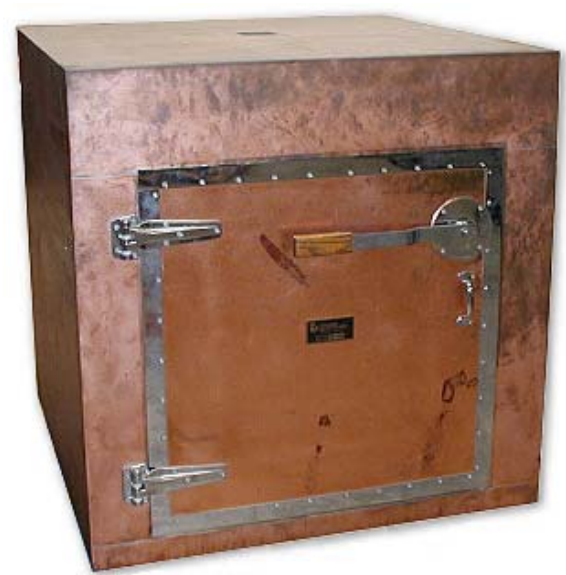

Figure 4.2.2: Lindgren Shielded 50-Inch RF-table enclosure. 


\subsection{Antenna Radiation Pattern Measurement}

This section introduces the radiation pattern setup and outlines what steps are taken for antenna pattern measurement. Because an anechoic chamber is not available at the time of this testing, there are several steps taken to eliminate ambient noise. First, a power amplifier is used to increase the network analyzer's transmitted signal strength to a value significantly larger than ambient noise levels. Second, multiple readings are averaged for each point of data. Third, an open environment free of moving objects is selected to minimize unwanted signal reflections. The equipment required for antenna pattern measurement is shown in Figure 2.1.1. The Antenna Under Test (AUT) is located on a Diamond Engineering Model 5000 antenna positioning system. An Agilent (Model HP8753A) HPIB-enabled network analyzer measures S21, power incident on port 2 with respect to power transmitted on port 1, to characterize antenna radiation pattern. A laptop running Desktop Antenna Measurement Studio (DAMS) automates AUT movement and stores received signal strength data using HPIB. The antenna positioning system is capable of controlling $0^{\circ}$ to $360^{\circ}$ horizontal rotation and $-45^{\circ}$ to $+45^{\circ}$ vertical position. Its platform movement is precisely controlled in $2.5^{\circ}$ horizontal steps and $5^{\circ}$ vertical steps resulting in 2592 points of data for each antenna. Three discrete S21 readings are averaged in each position for reduction of noise.
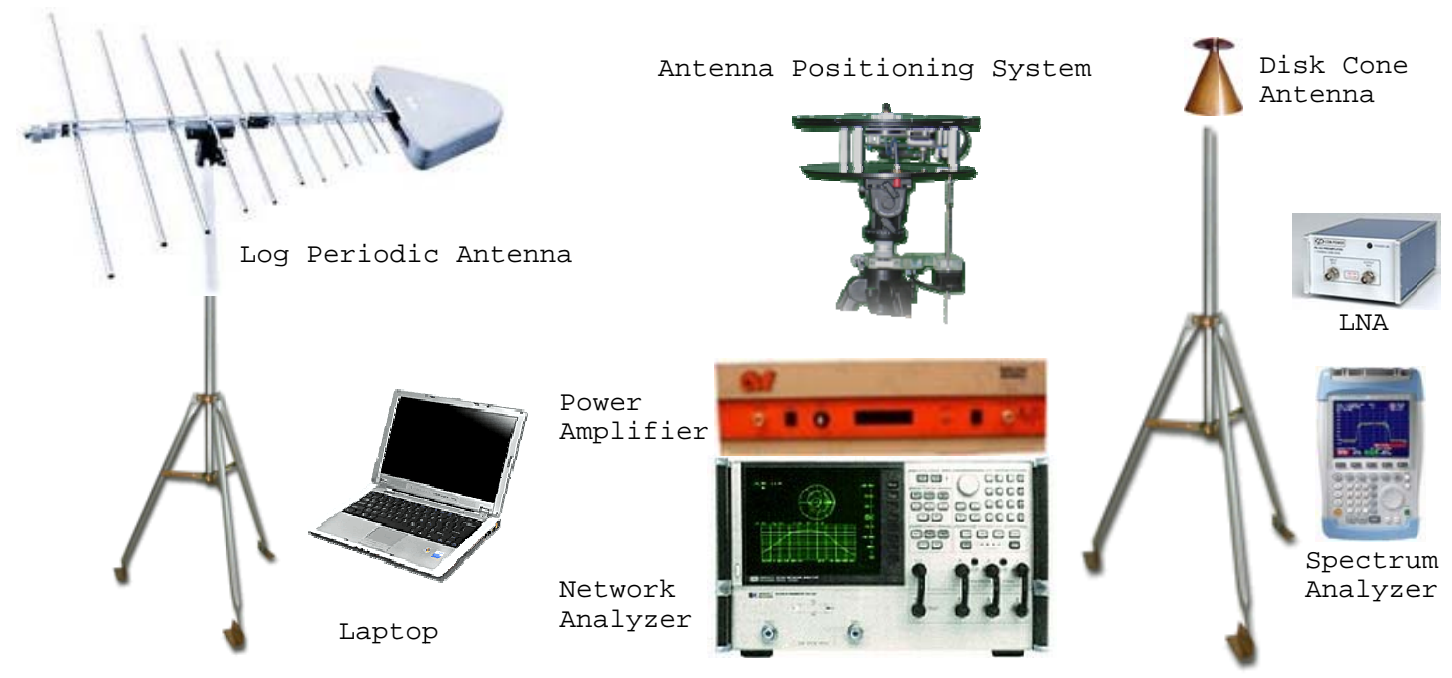

Figure 4.3.1: Equipment needed for radiation pattern measurement. 
Port 1 of the network analyzer is connected to an Amplifier Research (Model 30W1000B) power amplifier that drives a Lindgren (Model 3147) log periodic antenna. The log periodic antenna is positioned to transmit vertically polarized data. An adjustable power amplifier is set for $1 / 2$-watt output power increasing the signal-tonoise ratio of the network analyzer transmitted signal. Port 2 of the network analyzer is connected to the AUT and measures the incident received signal strength. The network analyzer is configured for $900-\mathrm{MHz}$ frequency with 5-MHz span and captures 51 points of data. A two-port round-trip "through" calibration is performed, compensating for all cable losses, linearity errors, and power amplification effects. The AUT and transmit antenna are separated by 15 feet to ensure the transmitted signal is sufficiently in the far-field region.

A second wide-band disk cone antenna is vertically mounted and continuously monitors the spectrum of interest. This antenna is connected to a Com Power (Model PA-103) Low Noise Amplifier (LNA) that offers 32-dB gain and is used to drive a Rohde and Schwarz (Model FSH3) spectrum analyzer. This provides the capability to measure background noise, as well as monitor network analyzer sweep rate and output power.

Measured data is post processed to remove path loss and transmit-antenna gain effects. The DAMS software provides a path loss calculator based on separation in feet and the Lindgren datasheet provides antenna gain measurements at the frequency of interest. Once corrected, the finalized data provides an accurate measurement for AUT gain. These finalized plots are presented in the prototype antenna measurement results Chapter 5. 


\section{Prototype-Antenna Measurement Results}

\subsection{Inverted-F Antenna}

\subsubsection{Antenna Orientation and Matching Circuit}

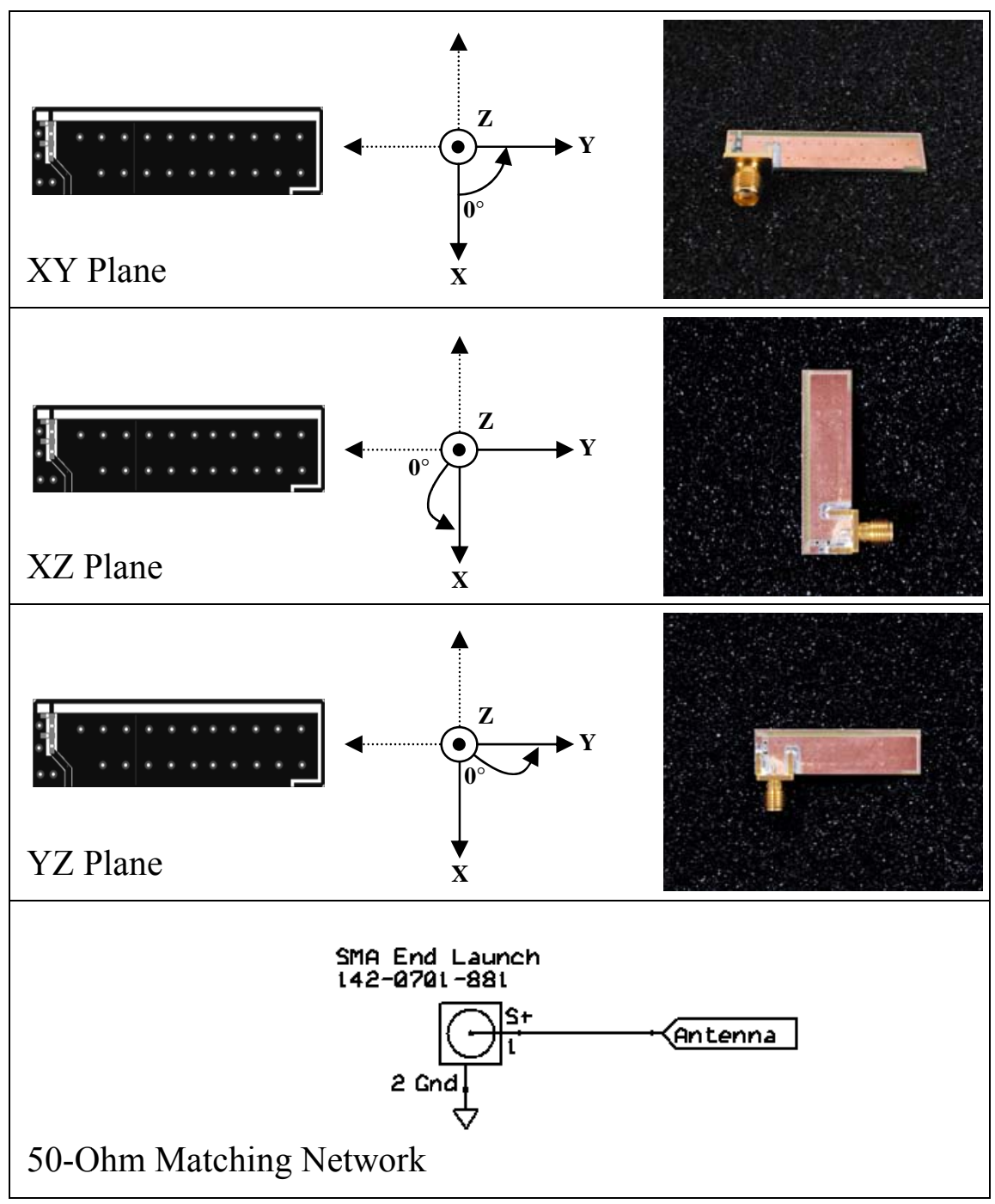

Figure 5.1.1: Inverted-F antenna orientation and 50-Ohm matching network. 


\subsubsection{D Radiation Pattern after Matching}

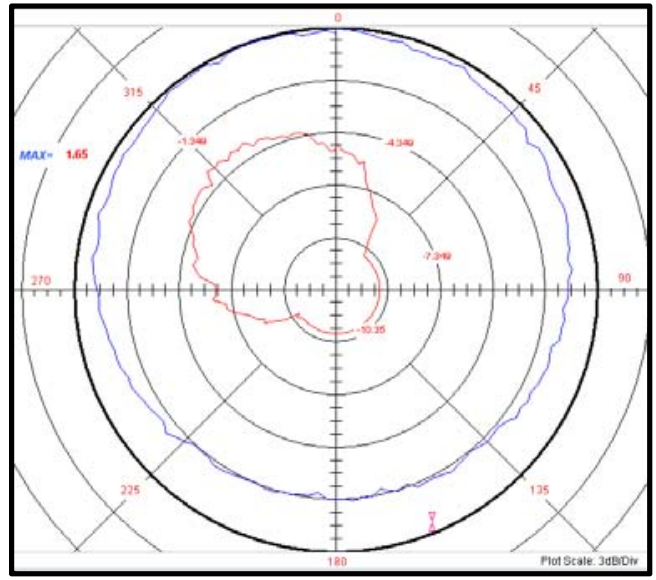

\begin{tabular}{l|ll|}
$\begin{array}{l}\text { Trace: } \mathbf{X Y} \\
\text { Plane }\end{array}$ & $\begin{array}{l}\text { Vertical } \\
\text { Polarization } \\
\text { (Blue) }\end{array}$ & $\begin{array}{l}\text { Horizontal } \\
\text { Polarization } \\
\text { (Red) }\end{array}$ \\
\hline $\begin{array}{l}\text { Frequency } \\
\text { Format }\end{array}$ & $\begin{array}{l}\text { 200 MHz } \\
\text { Azimuth }\end{array}$ & $\mathrm{AZ}=0-360 \mathrm{MHz}$ \\
Zenith & $\mathrm{Z}=90$ & $\mathrm{AZ}=0-360$ \\
Gain Min. & -1.734810 & $\mathrm{Z}=90$ \\
Gain Max. & 1.650669 & -18.004178 \\
Avg. Gain & 0.105123 & -9.152237
\end{tabular}

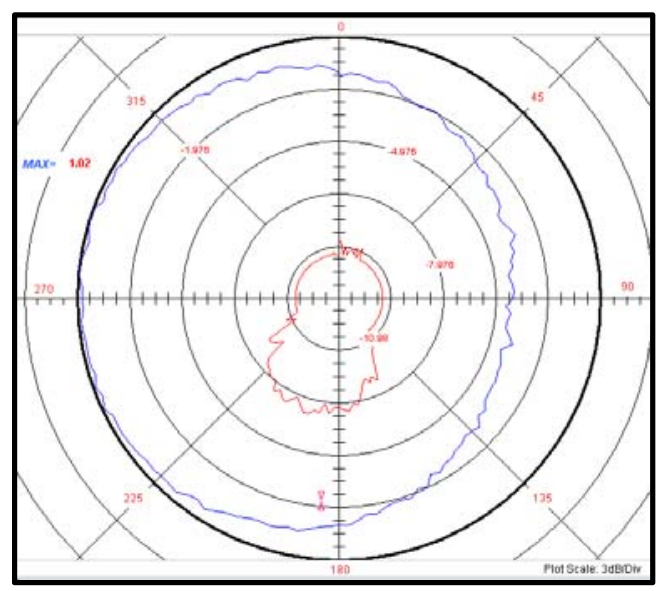

\begin{tabular}{l|ll|}
$\begin{array}{l}\text { Trace: } \mathbf{X Z} \\
\text { Plane }\end{array}$ & $\begin{array}{l}\text { Vertical } \\
\text { Polarization } \\
\text { (Blue) }\end{array}$ & $\begin{array}{l}\text { Horizontal } \\
\text { Polarization } \\
\text { (Red) }\end{array}$ \\
\hline $\begin{array}{l}\text { Frequency } \\
\text { Format }\end{array}$ & $\begin{array}{l}\text { 200 } \mathrm{MHz} \\
900 \mathrm{MHz}\end{array}$ \\
Azimuth & $\mathrm{AZ}=0$ & $20 \mathrm{Log}$ \\
Zenith & $\mathrm{Z}=0-360$ & $\mathrm{AZ}=0$ \\
Gain Min. & -4.435507 & -22.538322 \\
Gain Max. & 1.024120 & -7.159920 \\
Avg. Gain & -1.148956 & -11.989447
\end{tabular}

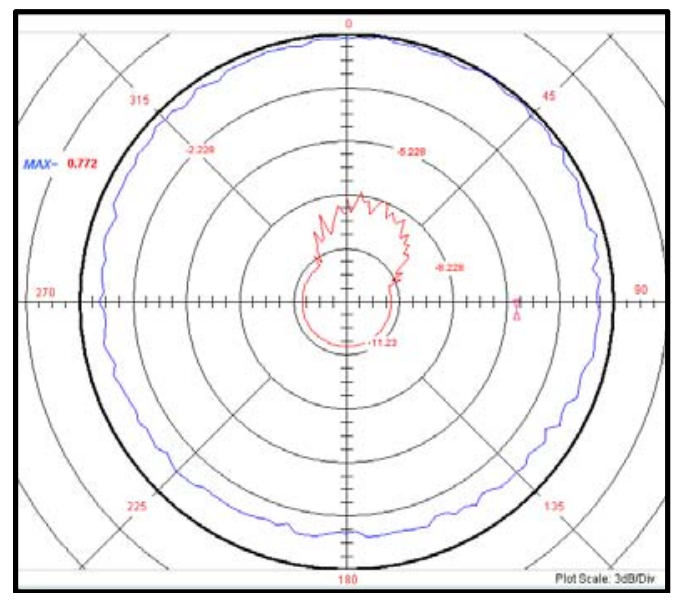

\begin{tabular}{l|ll|}
$\begin{array}{l}\text { Trace: YZ } \\
\text { Plane }\end{array}$ & $\begin{array}{l}\text { Vertical } \\
\text { Polarization } \\
\text { (Blue) }\end{array}$ & $\begin{array}{l}\text { Horizontal } \\
\text { Polarization } \\
\text { (Red) }\end{array}$ \\
\hline $\begin{array}{l}\text { Frequency } \\
\text { Format }\end{array}$ & $\begin{array}{l}\text { (B) MHz } \\
\text { Azimuth }\end{array}$ & $\mathrm{AZ}=90 \mathrm{MHz}$ \\
Zenith & $\mathrm{Z}=0-360$ & $\mathrm{Z}$ Log \\
Gain Min. & -1.389388 & -29.894365 \\
Gain Max. & 0.772073 & -8.027856 \\
Avg. Gain & -0.271077 & -14.194559
\end{tabular}

Figure 5.1.2: Inverted-F antenna 2D-radiation patterns expressed as gain in $\mathrm{dB}$ for $\mathrm{XY}, \mathrm{XZ}$, and YZ planes for horizontal and vertical polarization after addition of a matching network. 


\subsubsection{S11 Reflection Coefficient (dB)}

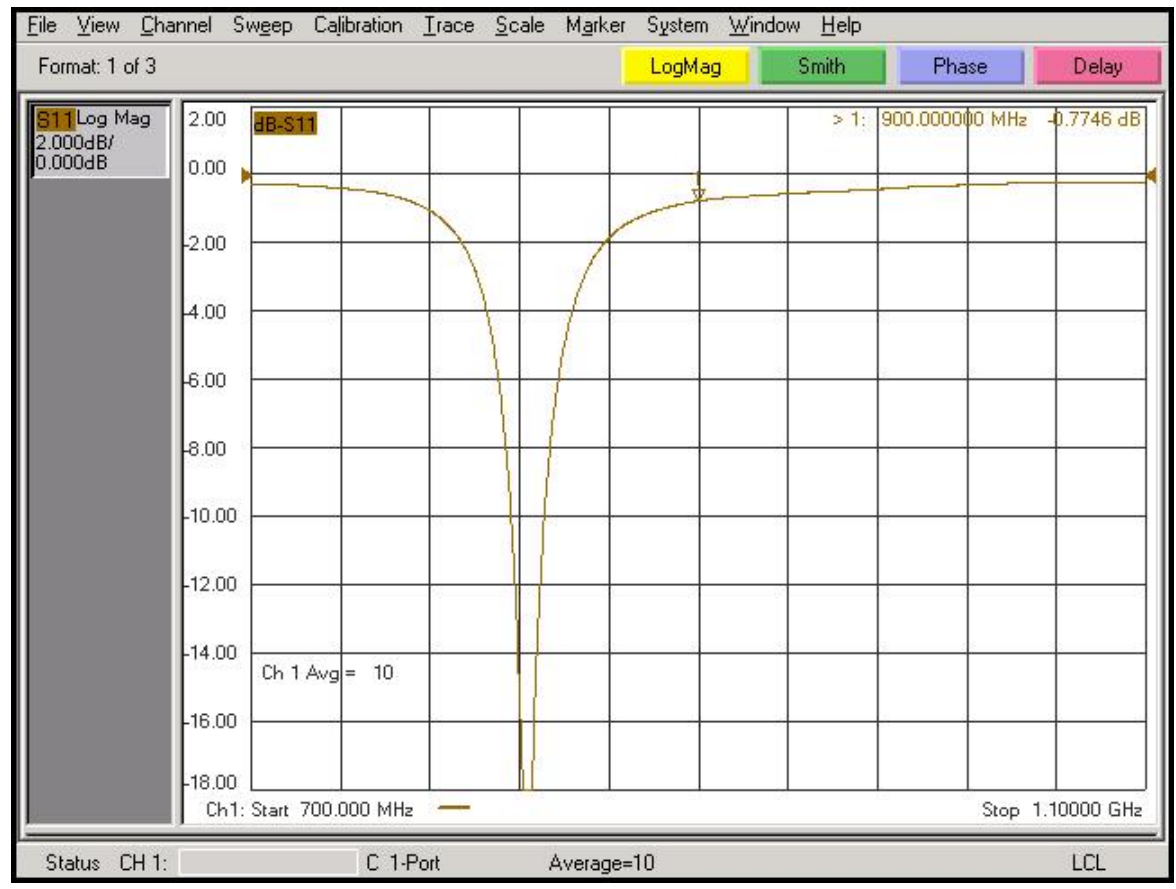

Figure 5.1.3: Inverted-F antenna input-port reflection coefficient (S11) expressed in dB before addition of a matching network.

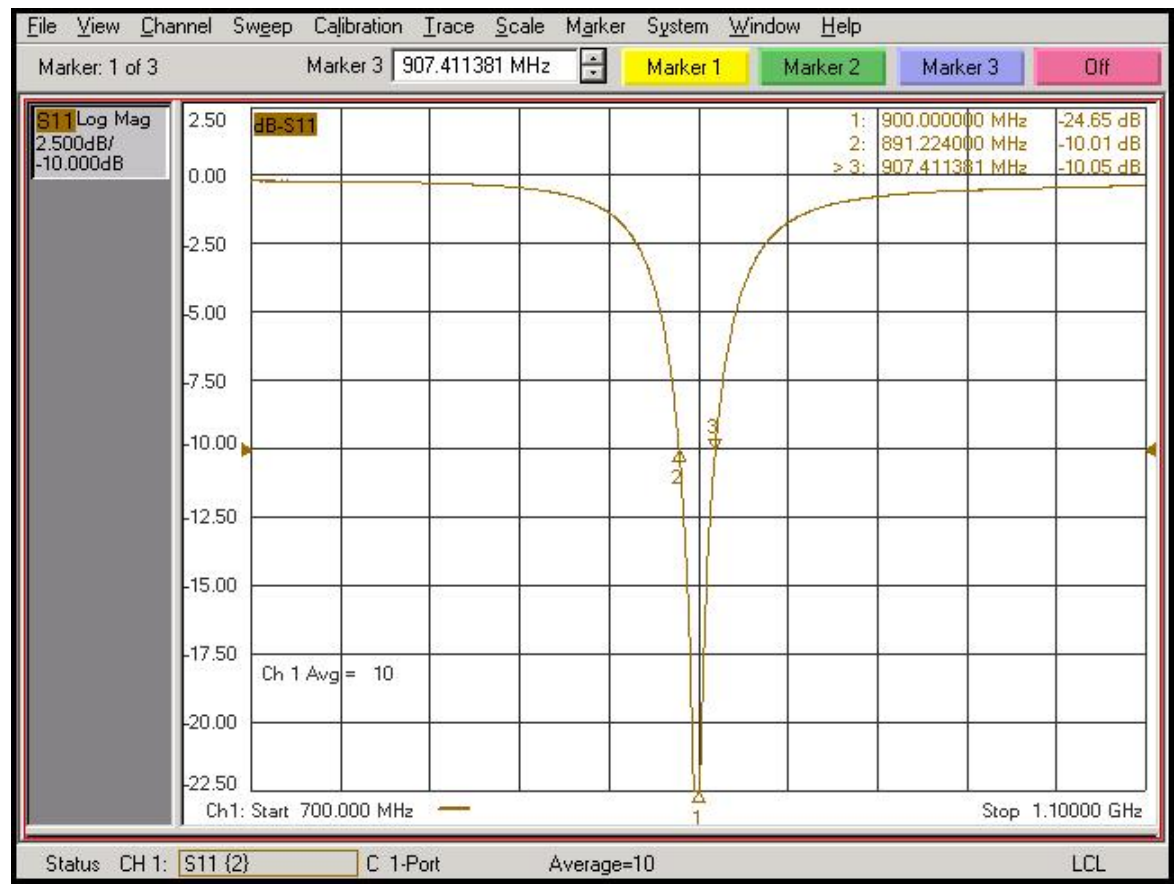

Figure 5.1.4: Inverted-F antenna input-port reflection coefficient (S11) expressed in dB after addition of a matching network. 


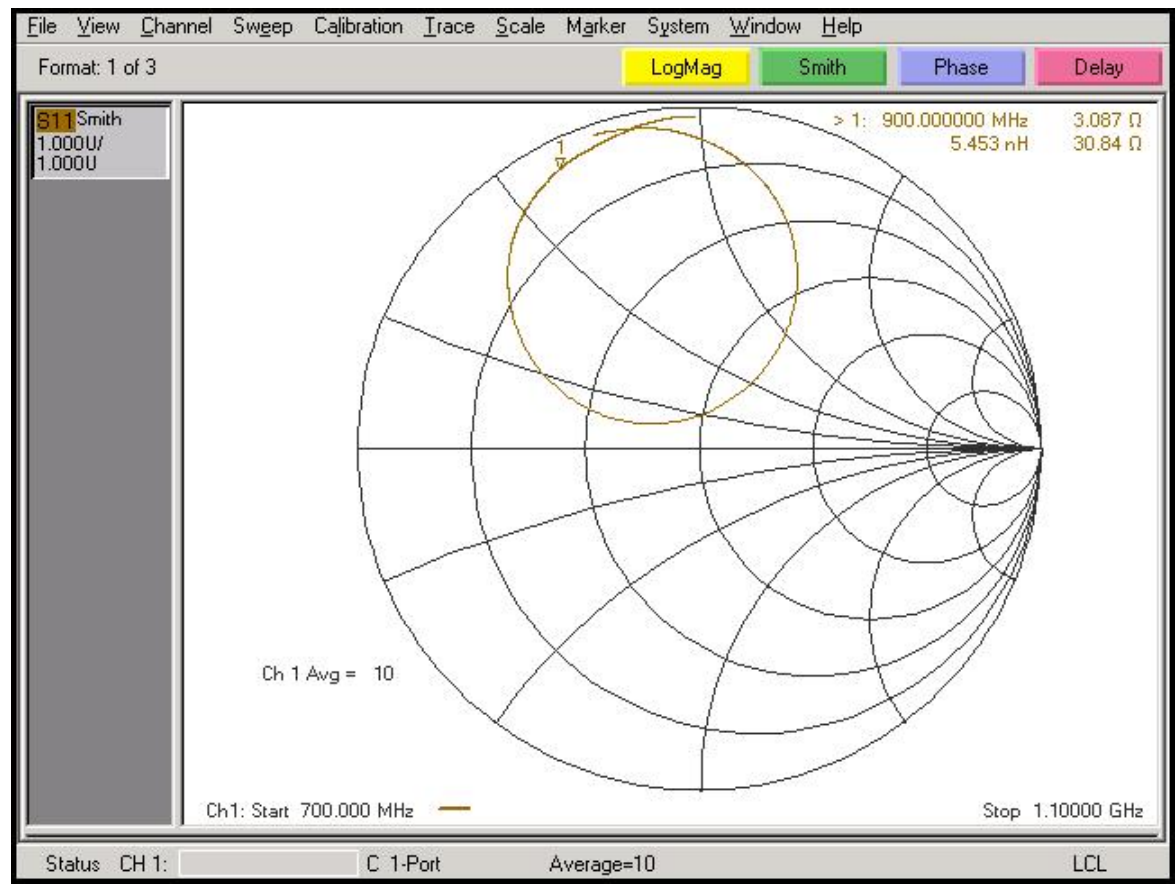

Figure 5.1.5: Inverted-F antenna input-port reflection coefficient (Smith Chart) before addition of a matching network.

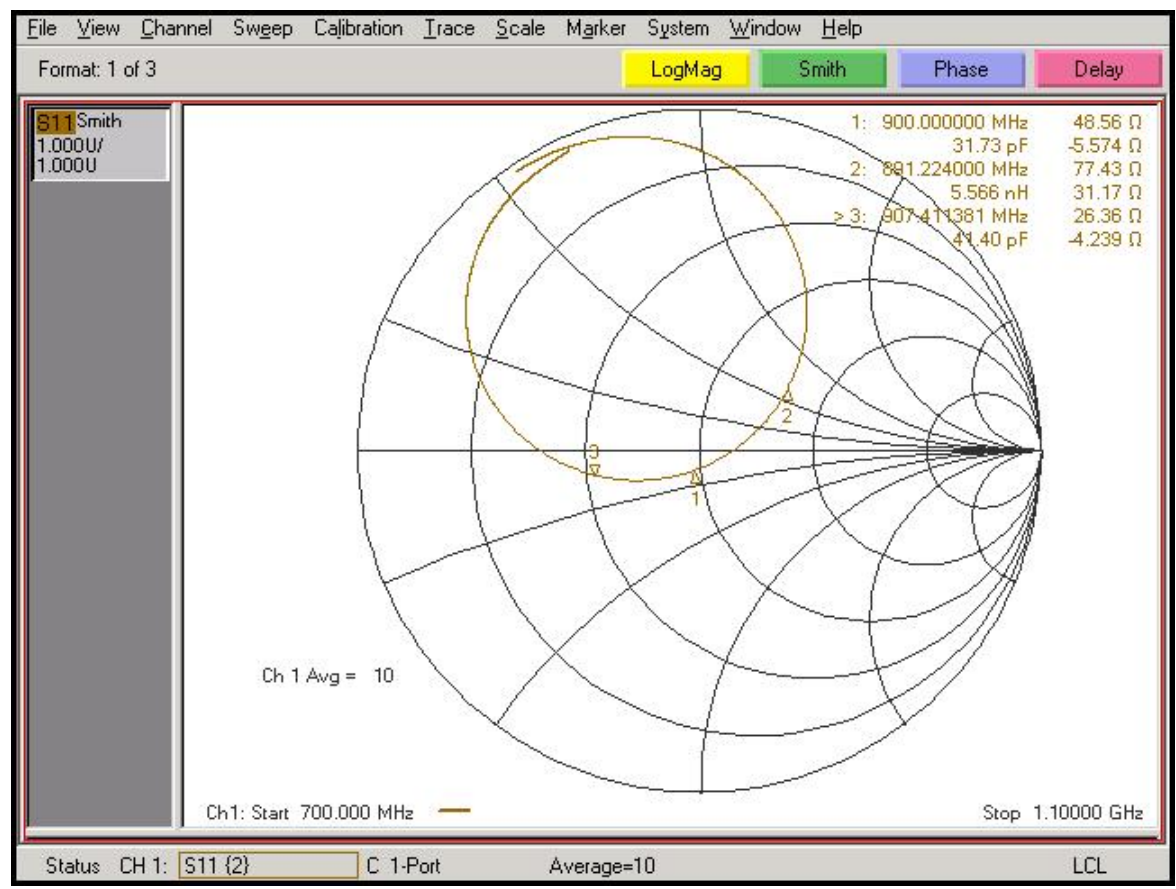

Figure 5.1.6: Inverted-F antenna input-port reflection coefficient (Smith Chart) after addition of a matching network. 


\subsubsection{Voltage Standing Wave Ratio (VSWR)}

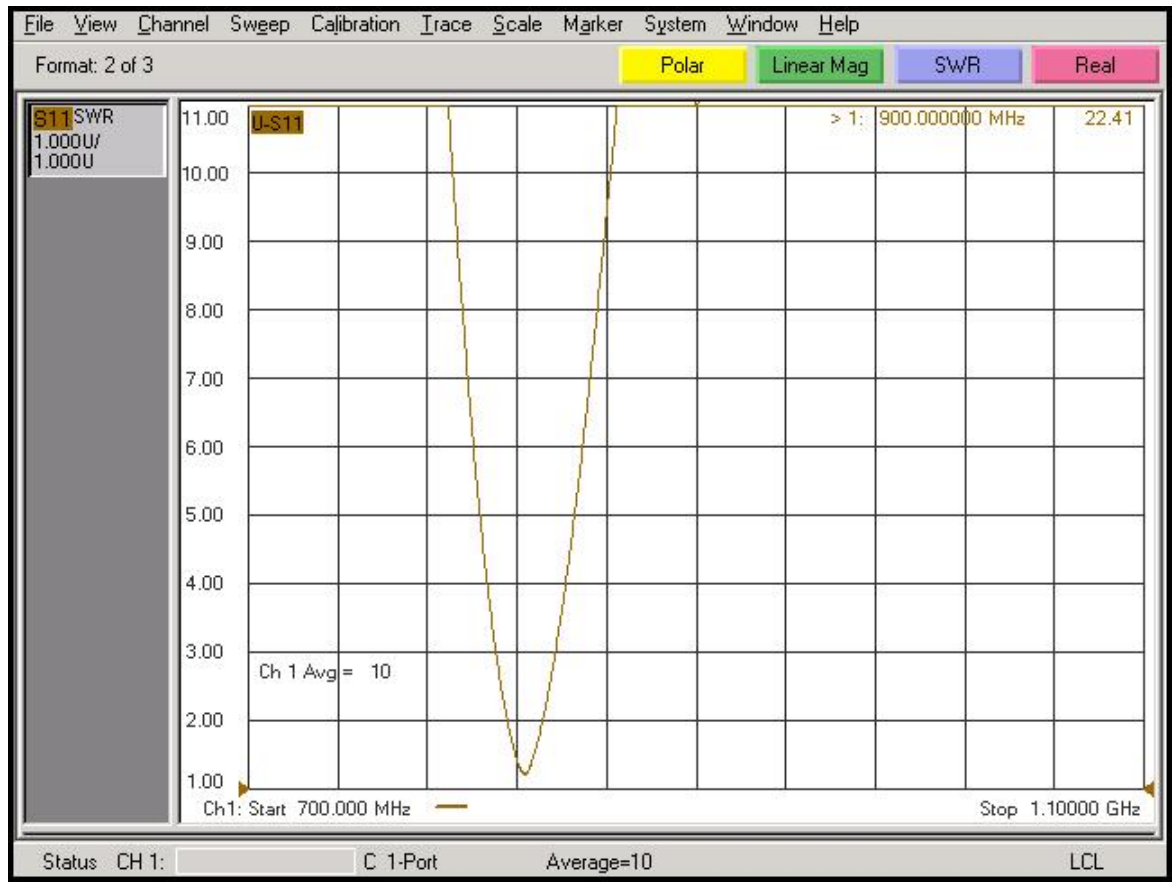

Figure 5.1.7: Inverted-F antenna input-port Voltage Standing Wave Ratio (VSWR) before addition of a matching network.

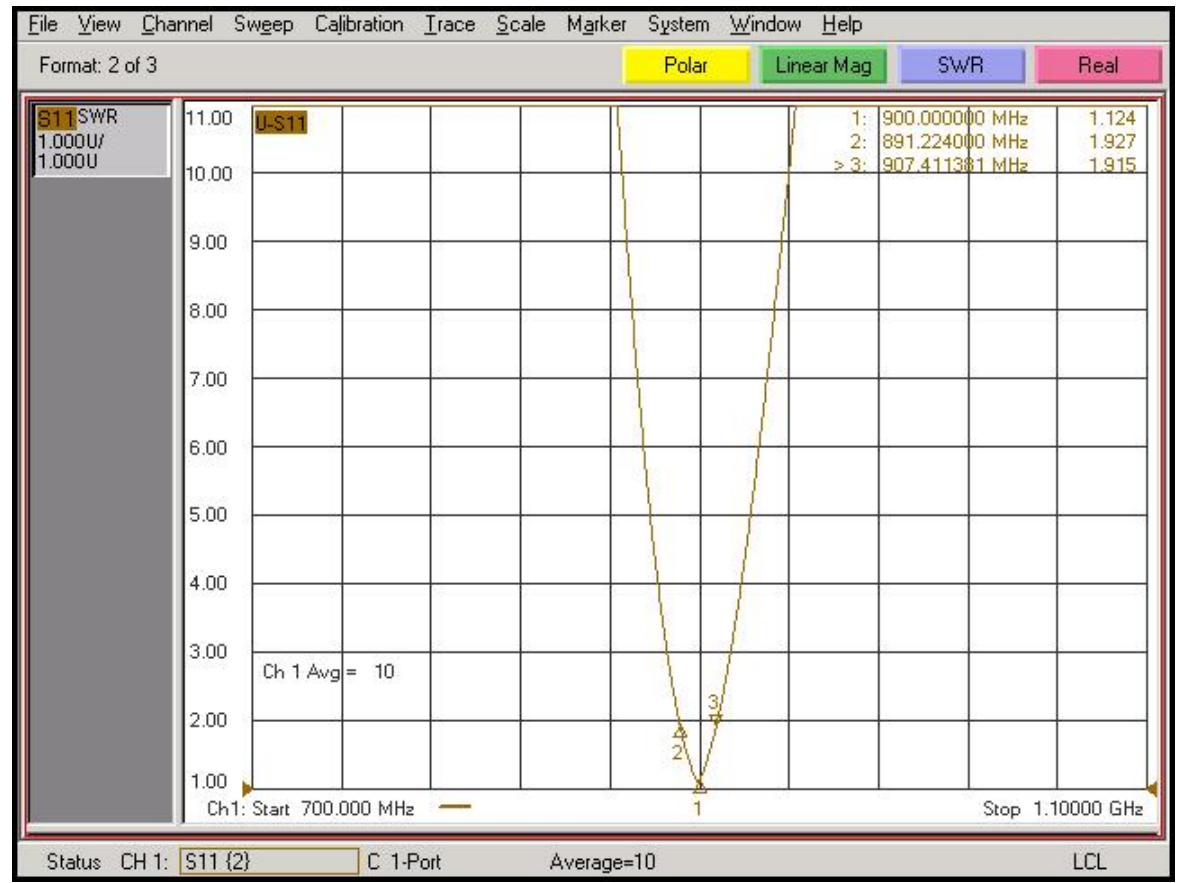

Figure 5.1.8: Inverted-F antenna input-port Voltage Standing Wave Ratio (VSWR) after addition of a matching network. 


\subsection{Long Meandering-Monopole Antenna}

\subsubsection{Antenna Orientation and Matching Circuit}

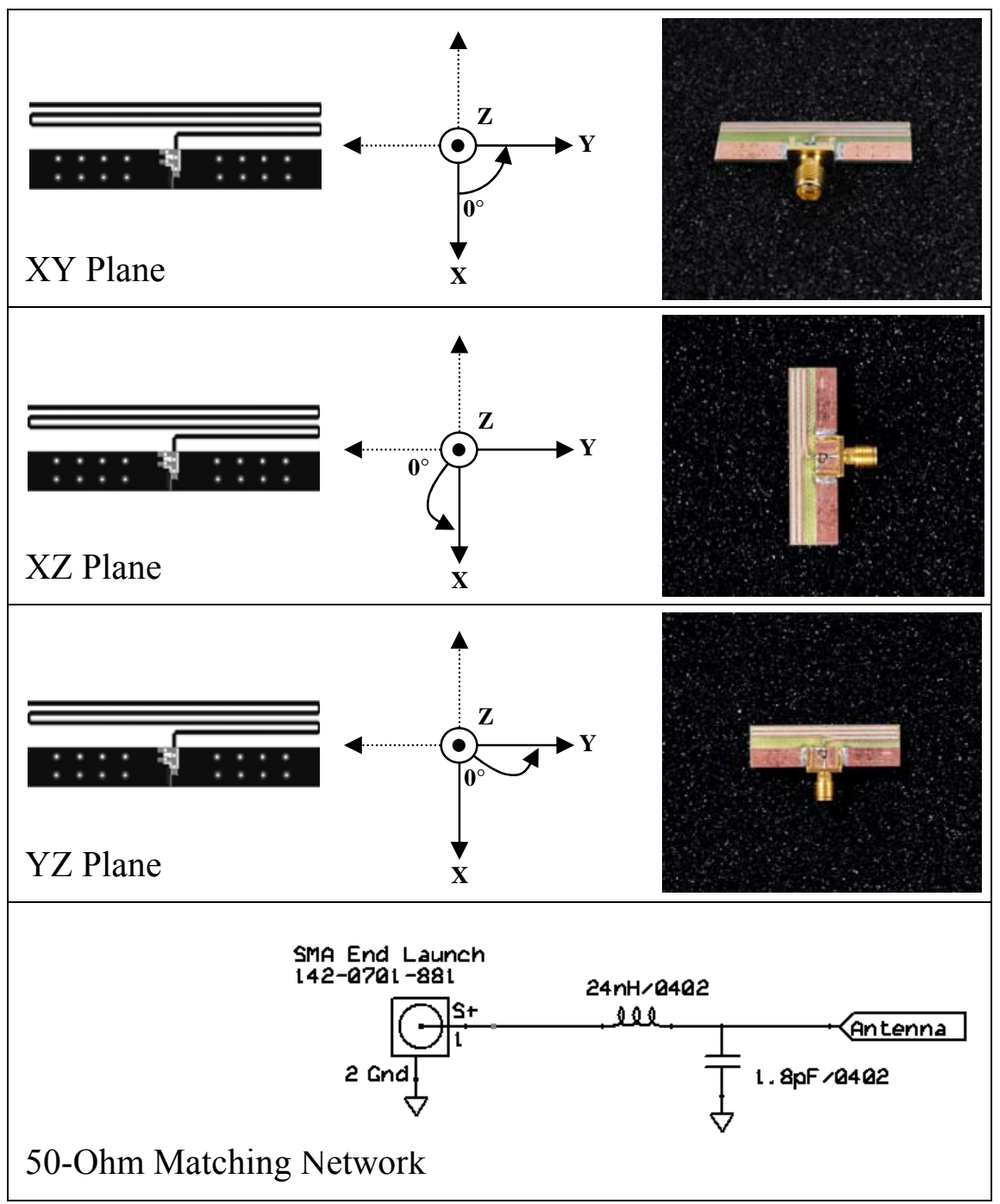

Figure 5.2.1: Long meandering-monopole orientation and 50-Ohm matching network. 


\subsubsection{D Radiation Pattern after Matching}

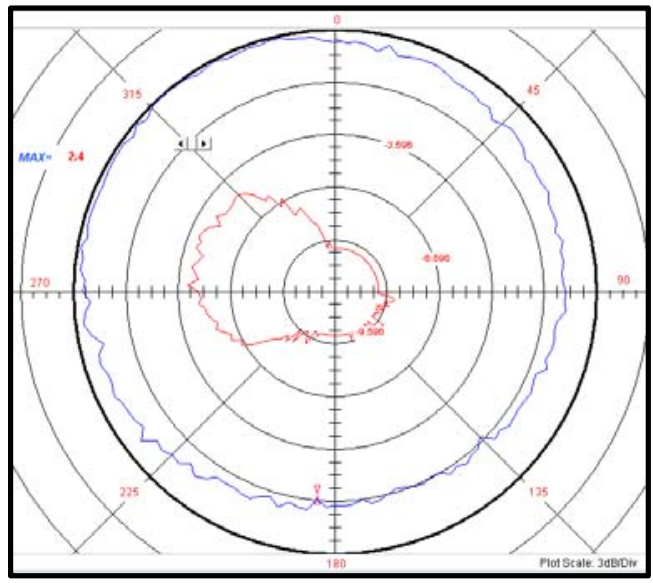

\begin{tabular}{|c|c|c|}
\hline $\begin{array}{l}\text { Trace: } X Y \\
\text { Plane }\end{array}$ & $\begin{array}{l}\text { Vertical } \\
\text { Polarization } \\
\text { (Blue) }\end{array}$ & $\begin{array}{l}\text { Horizontal } \\
\text { Polarization } \\
\text { (Red) }\end{array}$ \\
\hline Frequency & $900 \mathrm{MHz}$ & $900 \mathrm{MHz}$ \\
\hline Format & 20Log & 20Log \\
\hline Azimuth & $A Z=0-360$ & $A Z=0-360$ \\
\hline Zenith & $Z=90$ & $Z=90$ \\
\hline Gain Min. & -0.895065 & -24.208880 \\
\hline Gain Max. & 2.403603 & -4.133904 \\
\hline Avg. Gain & 0.947555 & -9.263607 \\
\hline
\end{tabular}

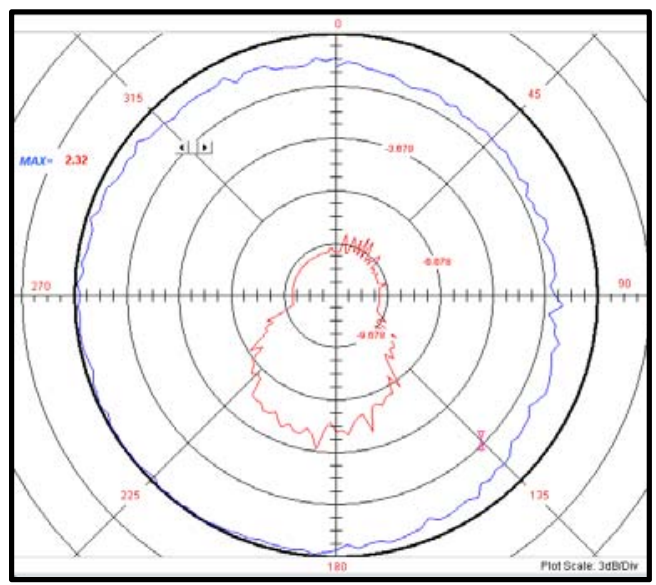

\begin{tabular}{l|ll|}
$\begin{array}{l}\text { Trace: } \mathbf{X Z} \\
\text { Plane }\end{array}$ & $\begin{array}{l}\text { Vertical } \\
\text { Polarization } \\
\text { (Blue) }\end{array}$ & $\begin{array}{l}\text { Horizontal } \\
\text { Polarization } \\
\text { (Red) }\end{array}$ \\
\hline $\begin{array}{l}\text { Frequency } \\
\text { Format }\end{array}$ & $\begin{array}{l}\text { 200 } \mathrm{MHz} \\
\text { Azimuth }\end{array}$ & $\mathrm{AZ}=0 \mathrm{MHz}$ \\
Zenith & $\mathrm{Z}=0-360$ & $\mathrm{AZ}=0$ \\
Gain Min. & -0.442871 & -25.520371 \\
Gain Max. & 2.321597 & -3.846894 \\
Avg. Gain & 1.124091 & -9.276315
\end{tabular}

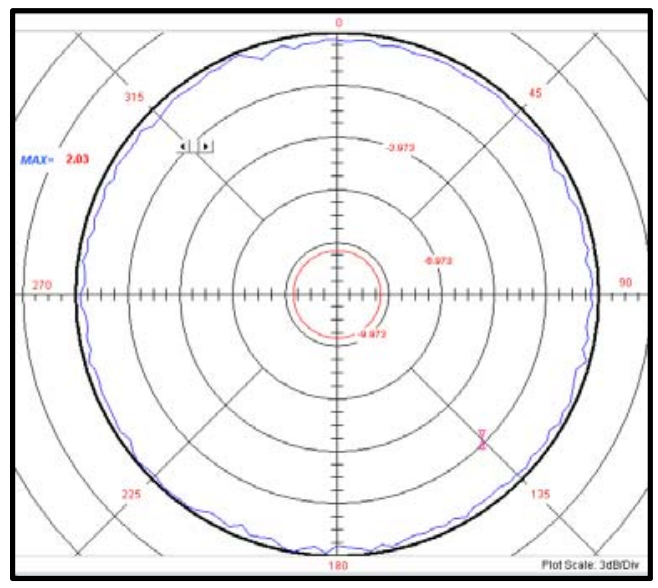

\begin{tabular}{|c|c|c|}
\hline $\begin{array}{l}\text { Trace: YZ } \\
\text { Plane }\end{array}$ & $\begin{array}{l}\text { Vertical } \\
\text { Polarization } \\
\text { (Blue) }\end{array}$ & $\begin{array}{l}\text { Horizontal } \\
\text { Polarization } \\
\text { (Red) }\end{array}$ \\
\hline Frequency & $900 \mathrm{MHz}$ & $900 \mathrm{MHz}$ \\
\hline Format & $20 \log$ & 20Log \\
\hline Azimuth & $A Z=90$ & $A Z=90$ \\
\hline Zenith & $Z=0-360$ & $Z=0-360$ \\
\hline Gain Min. & 1.117629 & -16.713373 \\
\hline Gain Max. & 2.026826 & -11.350361 \\
\hline Avg. Gain & 1.588872 & -13.773913 \\
\hline
\end{tabular}

Figure 5.2.2: Long meandering-monopole 2D-radiation pattern expressed as gain in $\mathrm{dB}$ for $\mathrm{XY}$, $\mathrm{XZ}$, and YZ planes after addition of a matching network. 


\subsubsection{S11 Reflection Coefficient (dB)}

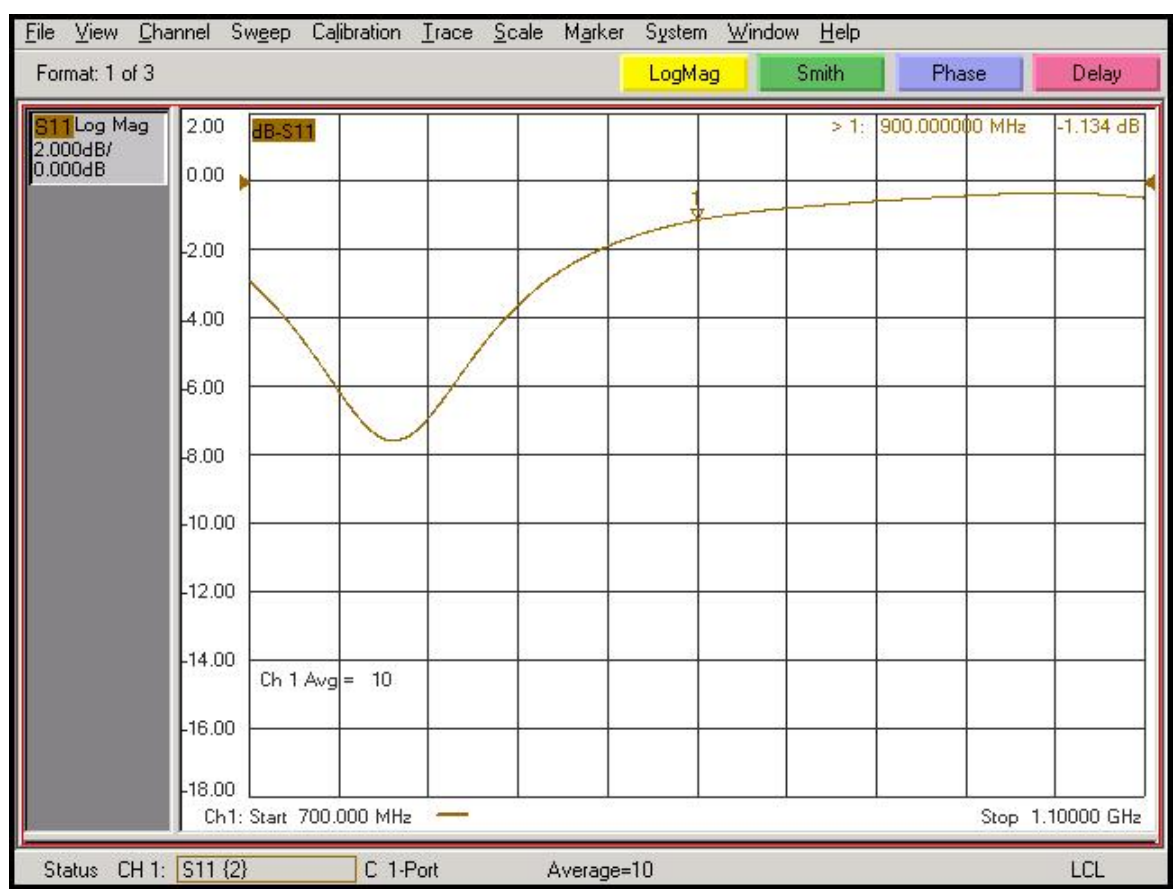

Figure 5.2.3: Long meandering-monopole input-port reflection coefficient (S11) expressed in $\mathrm{dB}$ before addition of a matching network.

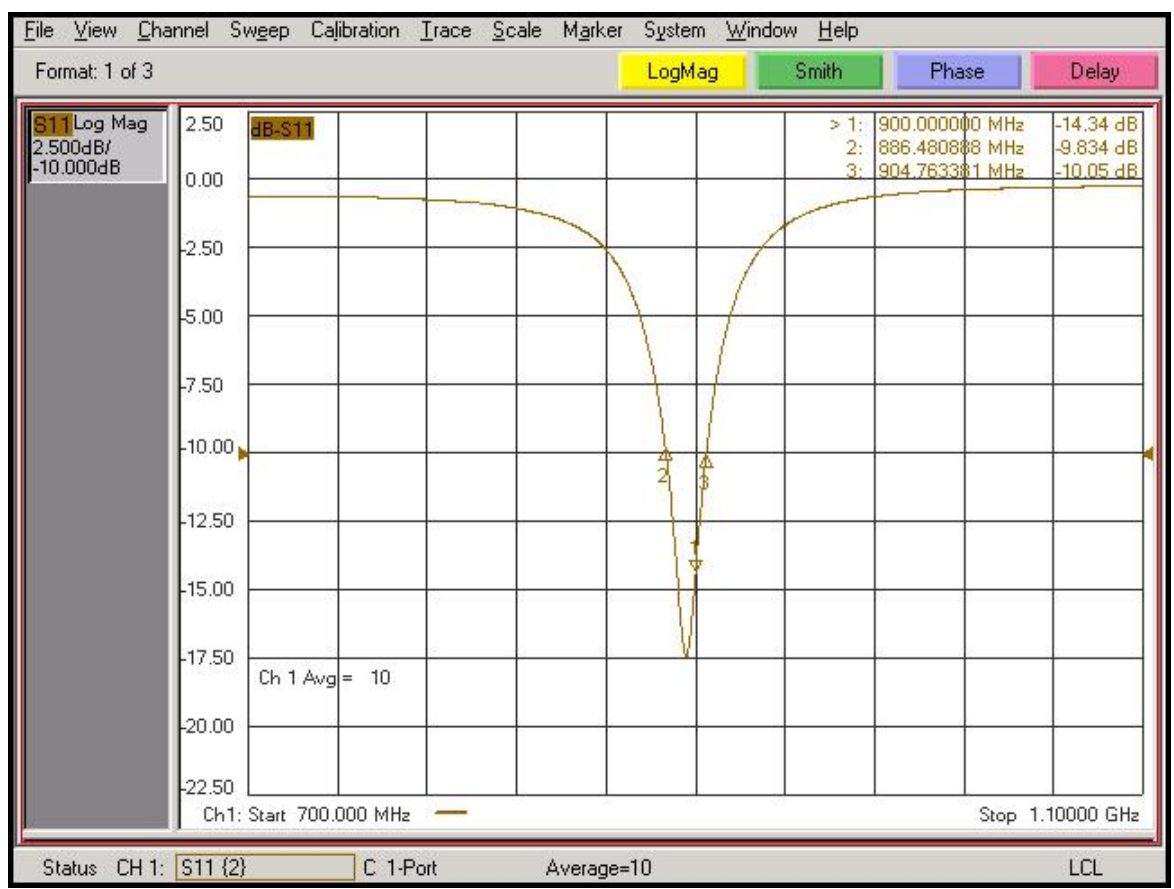

Figure 5.2.4: Long meandering-monopole input-port reflection coefficient (S11) expressed in $\mathrm{dB}$ after addition of a matching network. 


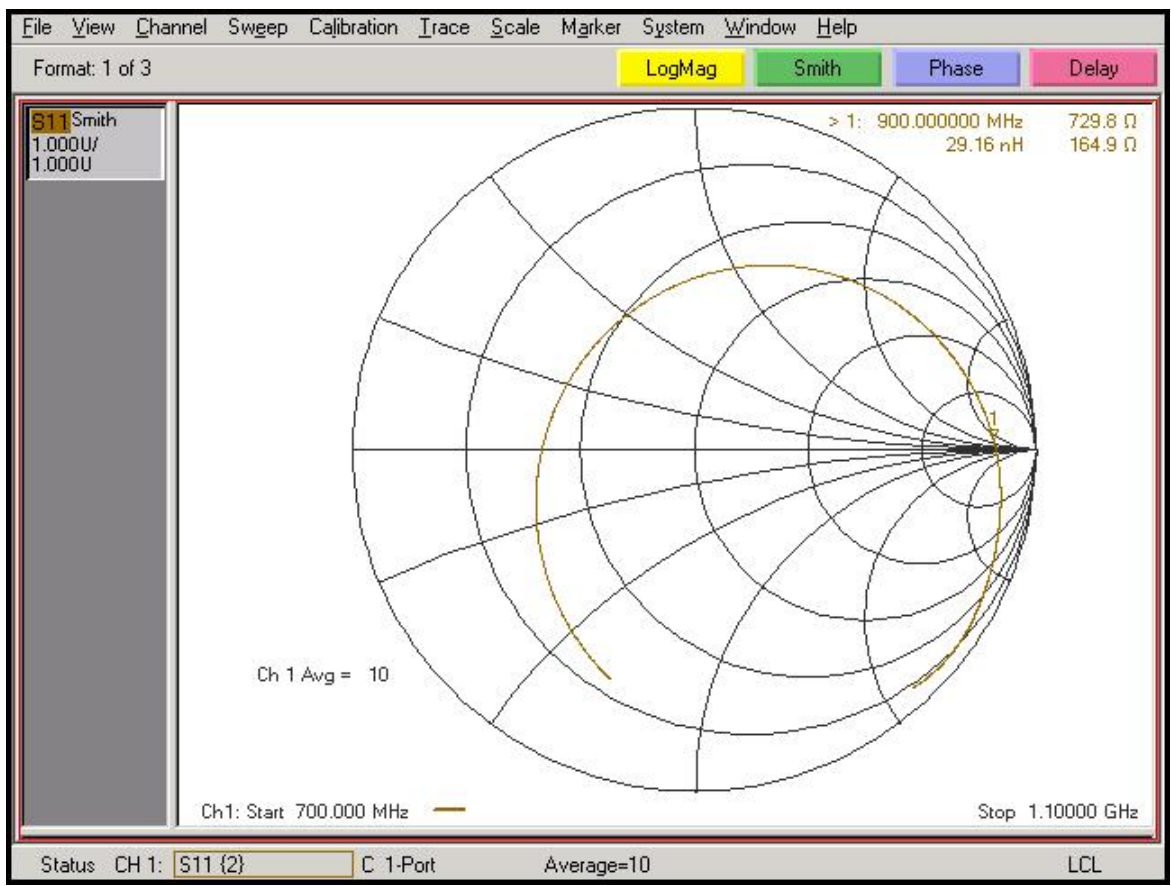

Figure 5.2.5: Long meandering-monopole input-port reflection coefficient (Smith Chart) before addition of a matching network.

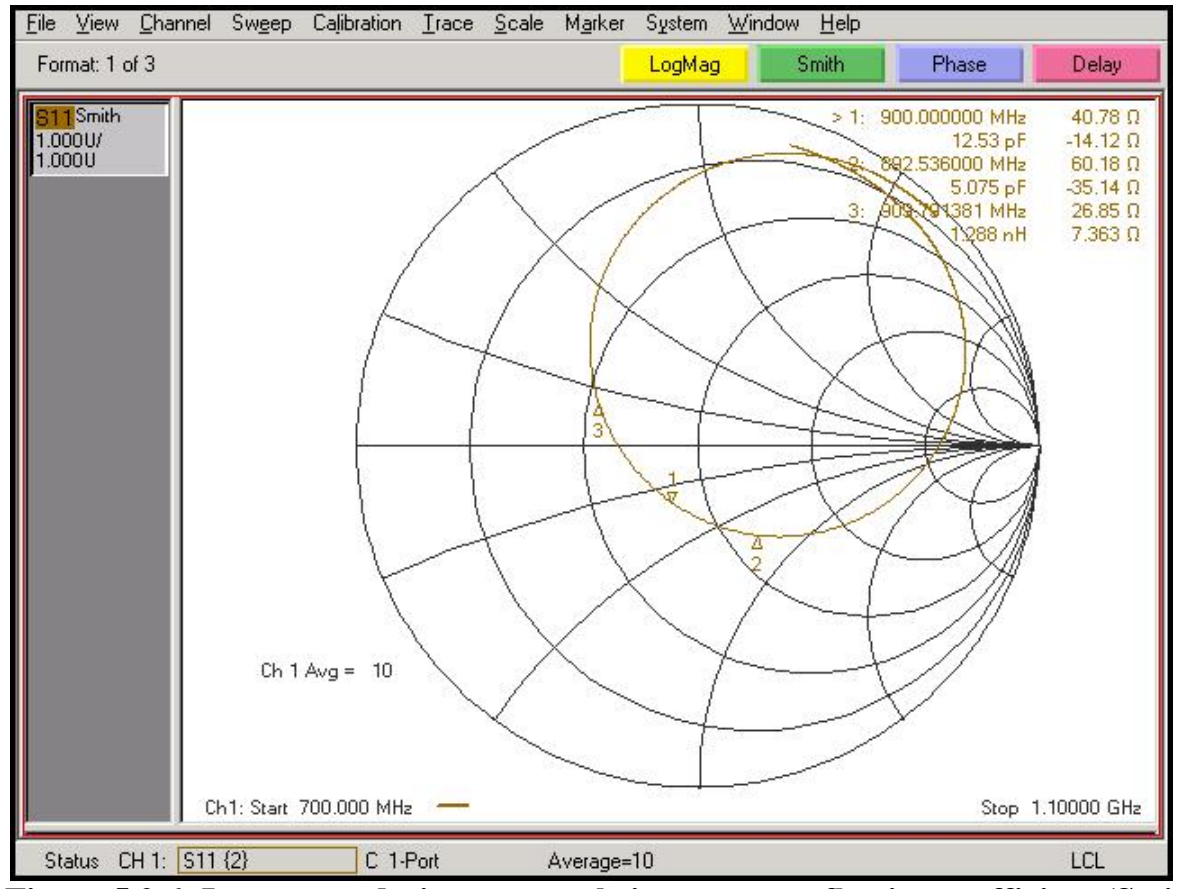

Figure 5.2.6: Long meandering-monopole input-port reflection coefficient (Smith Chart) after addition of a matching network. 


\subsubsection{Voltage Standing Wave Ratio (VSWR)}

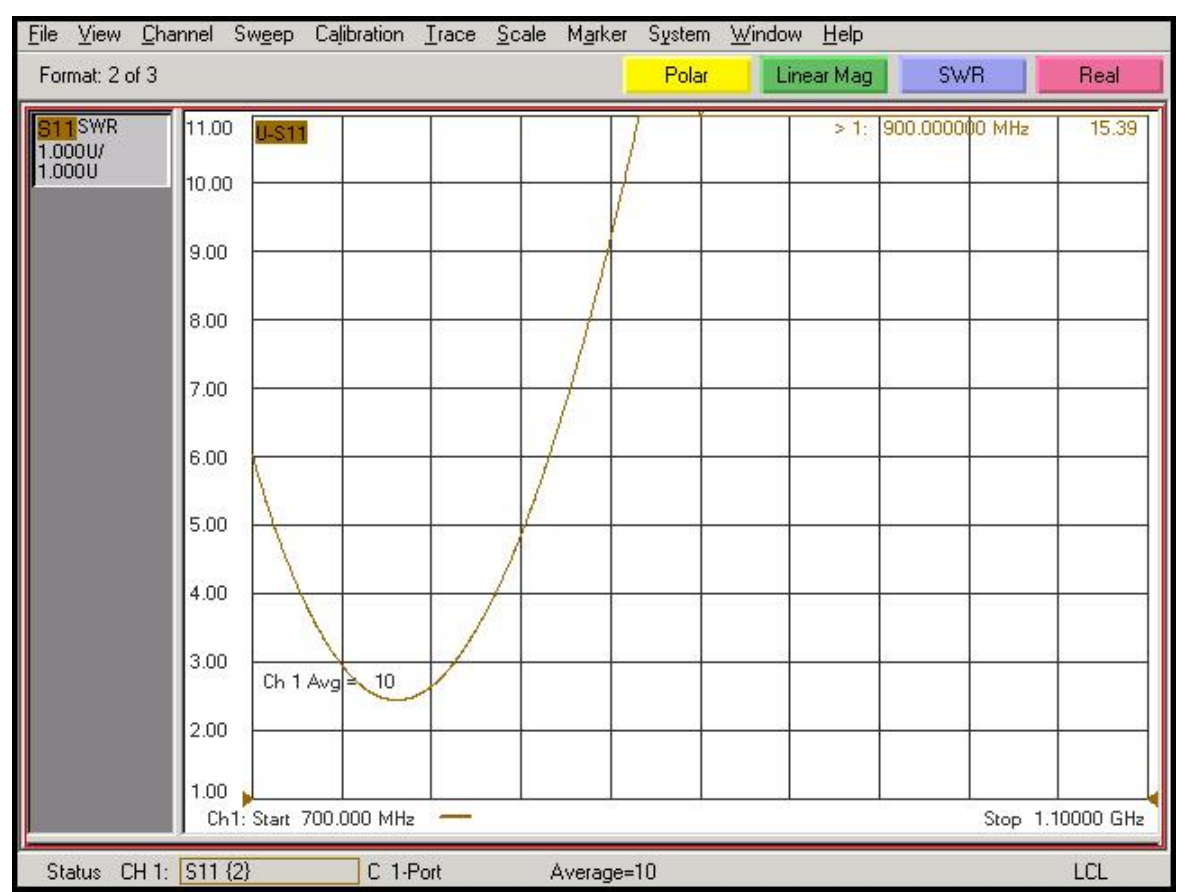

Figure 5.2.7: Long meandering-monopole input-port Voltage Standing Wave Ratio (VSWR) before addition of a matching network.

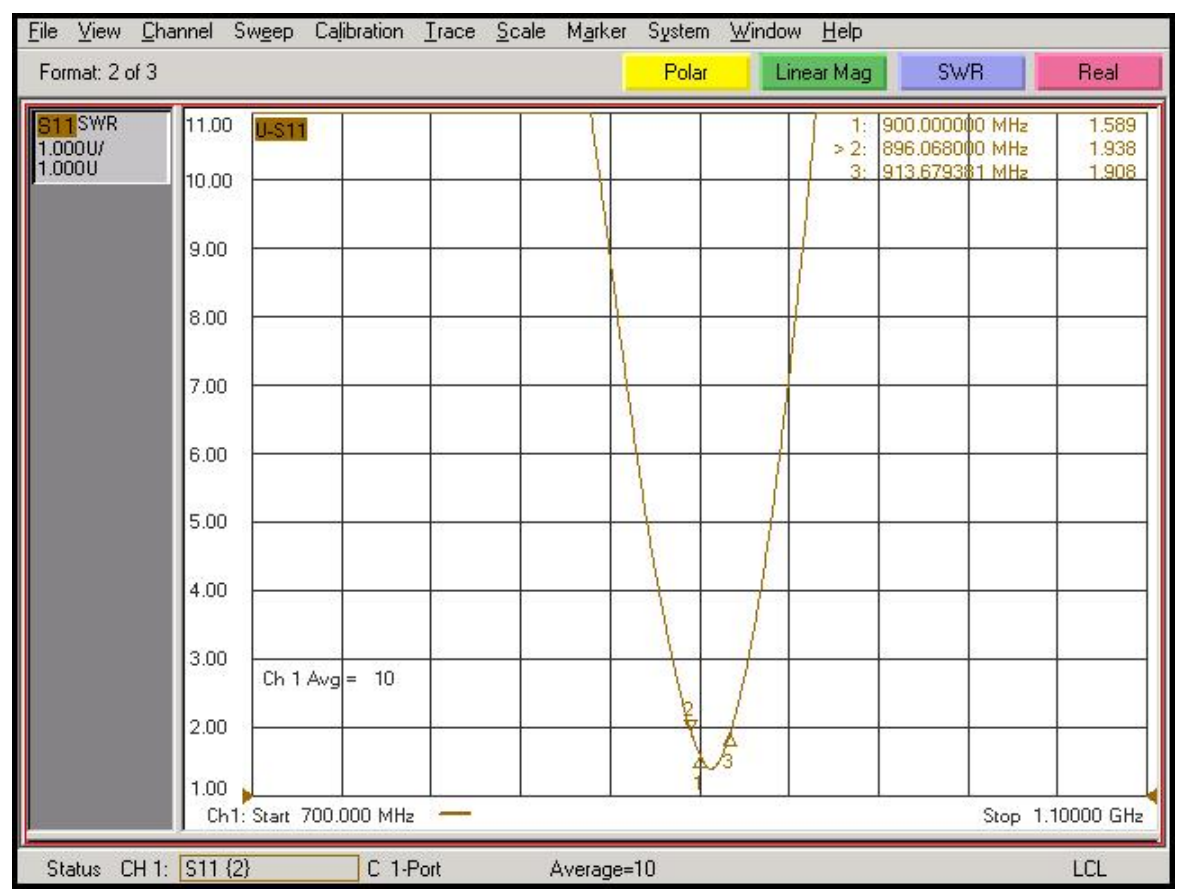

Figure 5.2.8: Long meandering-monopole input-port Voltage Standing Wave Ratio (VSWR) after addition of a matching network. 


\subsection{Short Meandering-Monopole Antenna}

\subsubsection{Antenna Orientation and Matching Circuit}

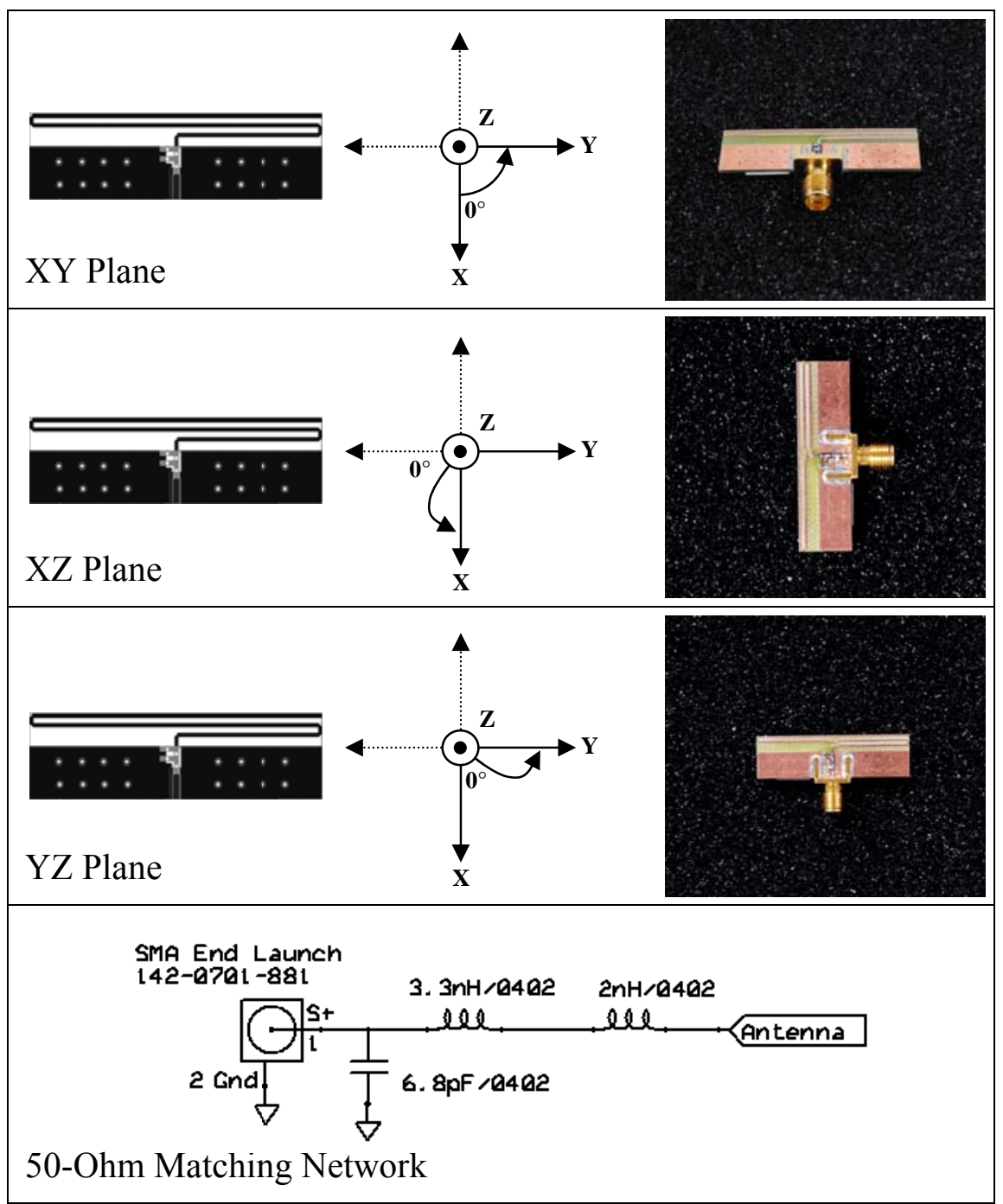

Figure 5.3.1: Short meandering-monopole orientation and 50-Ohm matching network. 


\subsubsection{D Radiation Pattern after Matching}

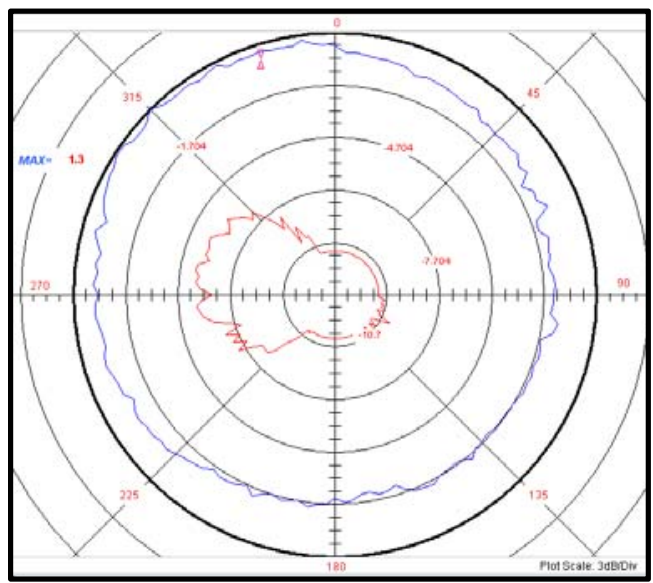

\begin{tabular}{|c|c|c|}
\hline $\begin{array}{l}\text { Trace: } \mathbf{X Y} \\
\text { Plane }\end{array}$ & $\begin{array}{l}\text { Vertical } \\
\text { Polarization } \\
\text { (Blue) }\end{array}$ & $\begin{array}{l}\text { Horizontal } \\
\text { Polarization } \\
\text { (Red) }\end{array}$ \\
\hline Frequency & $900 \mathrm{MHz}$ & $900 \mathrm{MHz}$ \\
\hline Format & 20Log & 20Log \\
\hline Azimuth & $A Z=0-360$ & $A Z=0-360$ \\
\hline Zenith & $Z=90$ & $Z=90$ \\
\hline Gain Min. & -2.314526 & -27.973085 \\
\hline Gain Max. & 1.296417 & -5.618204 \\
\hline Avg. Gain & -0.568787 & -11.062150 \\
\hline
\end{tabular}

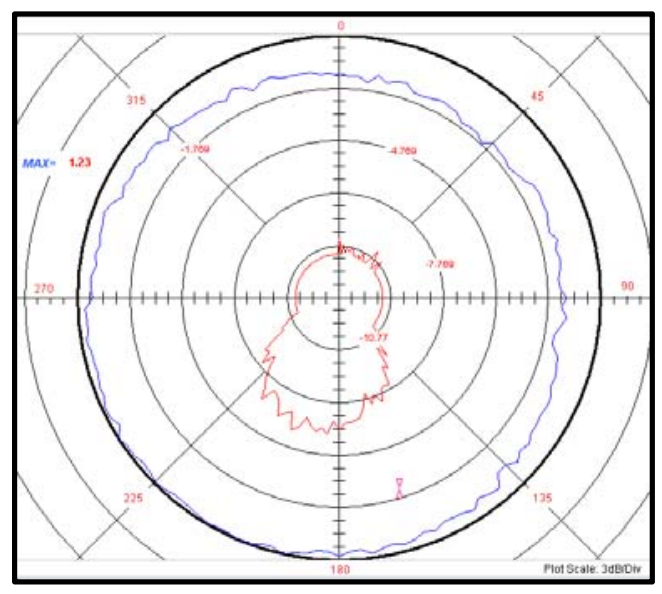

\begin{tabular}{|c|c|c|}
\hline $\begin{array}{l}\text { Trace: XZ } \\
\text { Plane }\end{array}$ & $\begin{array}{l}\text { Vertical } \\
\text { Polarization } \\
\text { (Blue) }\end{array}$ & $\begin{array}{l}\text { Horizontal } \\
\text { Polarization } \\
\text { (Red) }\end{array}$ \\
\hline Frequency & $900 \mathrm{MHz}$ & $900 \mathrm{MHz}$ \\
\hline Format & 20Log & 20Log \\
\hline Azimuth & $A Z=0$ & $A Z=0$ \\
\hline Zenith & $Z=0-360$ & $Z=0-360$ \\
\hline Gain Min. & -1.768155 & -26.256288 \\
\hline Gain Max. & 1.230869 & -5.740235 \\
\hline Avg. Gain & -0.095202 & -11.271322 \\
\hline
\end{tabular}

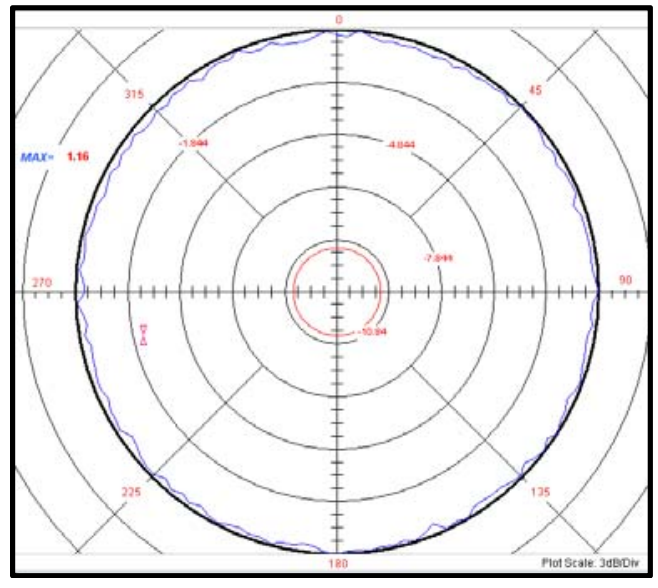

\begin{tabular}{lll|}
$\begin{array}{l}\text { Trace: YZ } \\
\text { Plane }\end{array}$ & $\begin{array}{l}\text { Vertical } \\
\text { Polarization } \\
\text { (Blue) }\end{array}$ & $\begin{array}{l}\text { Horizontal } \\
\text { Polarization } \\
\text { (Red) }\end{array}$ \\
\hline $\begin{array}{l}\text { Frequency } \\
\text { Format }\end{array}$ & $900 \mathrm{MHz}$ & $900 \mathrm{MHz}$ \\
Azimuth & AZ $=90$ & AZ \\
Zenith & $\mathrm{Z}=0-360$ & $\mathrm{Z}=0-360$ \\
Gain Min. & 0.502043 & -21.414459 \\
Gain Max. & 1.155939 & -12.924029 \\
Avg. Gain & 0.816280 & -16.106303
\end{tabular}

Figure 5.3.2: Short meandering-monopole 2D-radiation pattern expressed as gain in $\mathrm{dB}$ for $\mathrm{XY}$, $\mathrm{XZ}$, and YZ planes after addition of a matching network. 


\subsubsection{S11 Reflection Coefficient (dB)}

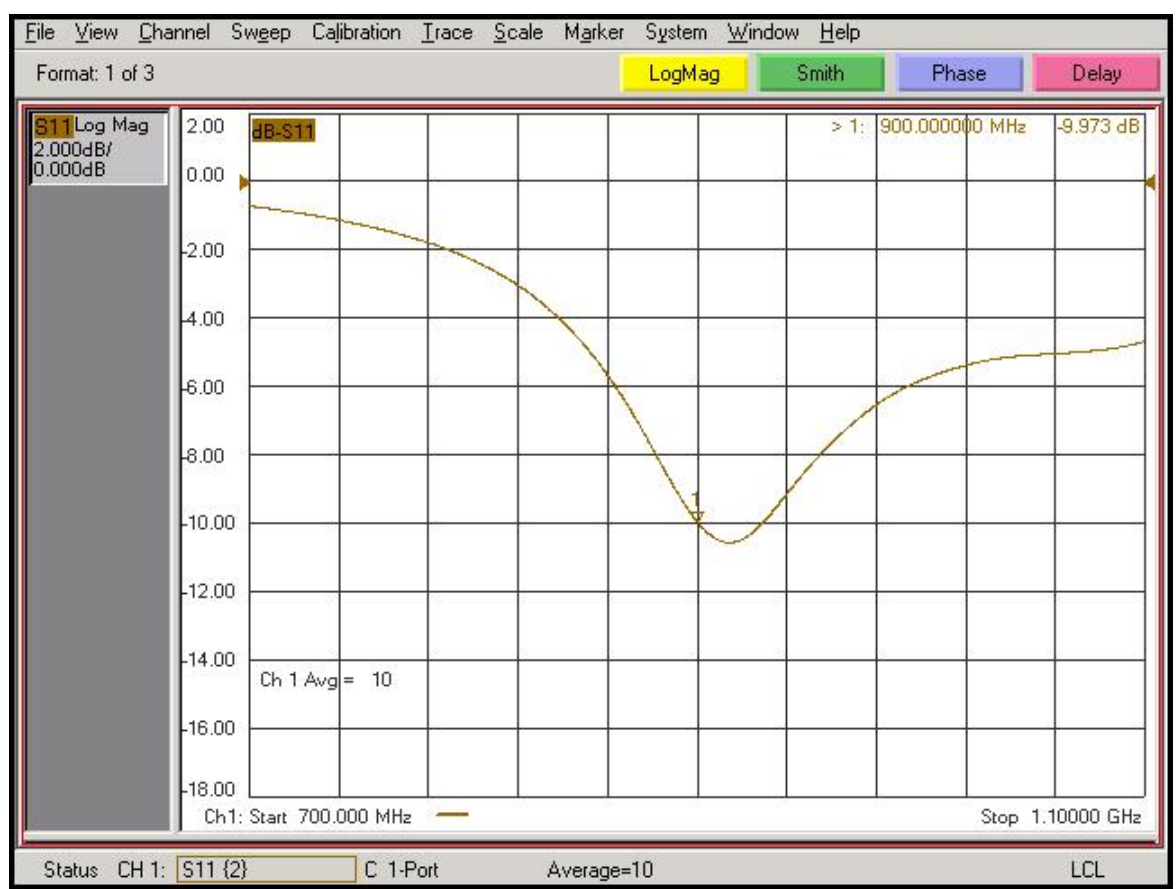

Figure 5.3.3: Short meandering-monopole input-port reflection coefficient (S11) expressed in $\mathrm{dB}$ before addition of a matching network.

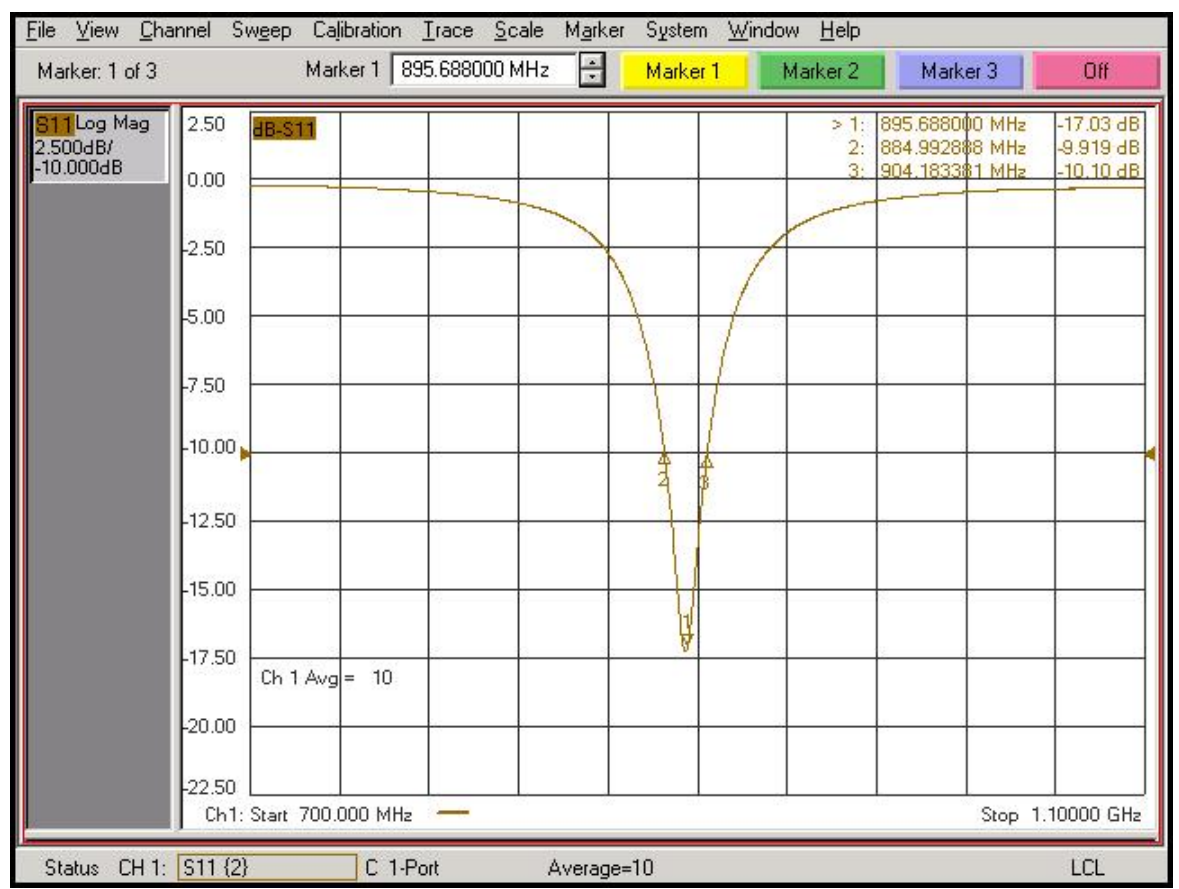

Figure 5.3.4: Short meandering-monopole input-port reflection coefficient (S11) expressed in $\mathrm{dB}$ after addition of a matching network. 


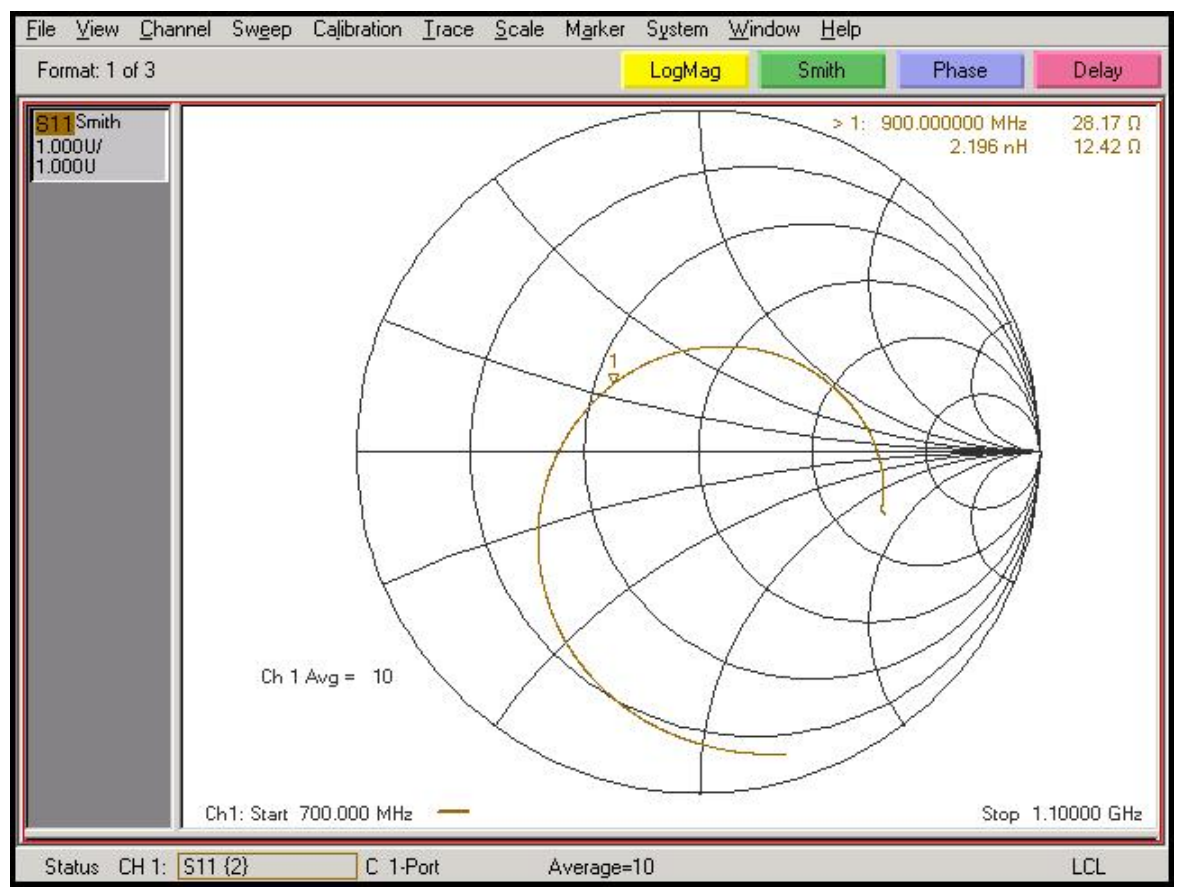

Figure 5.3.5: Short meandering-monopole input-port reflection coefficient (Smith Chart) before addition of a matching network.

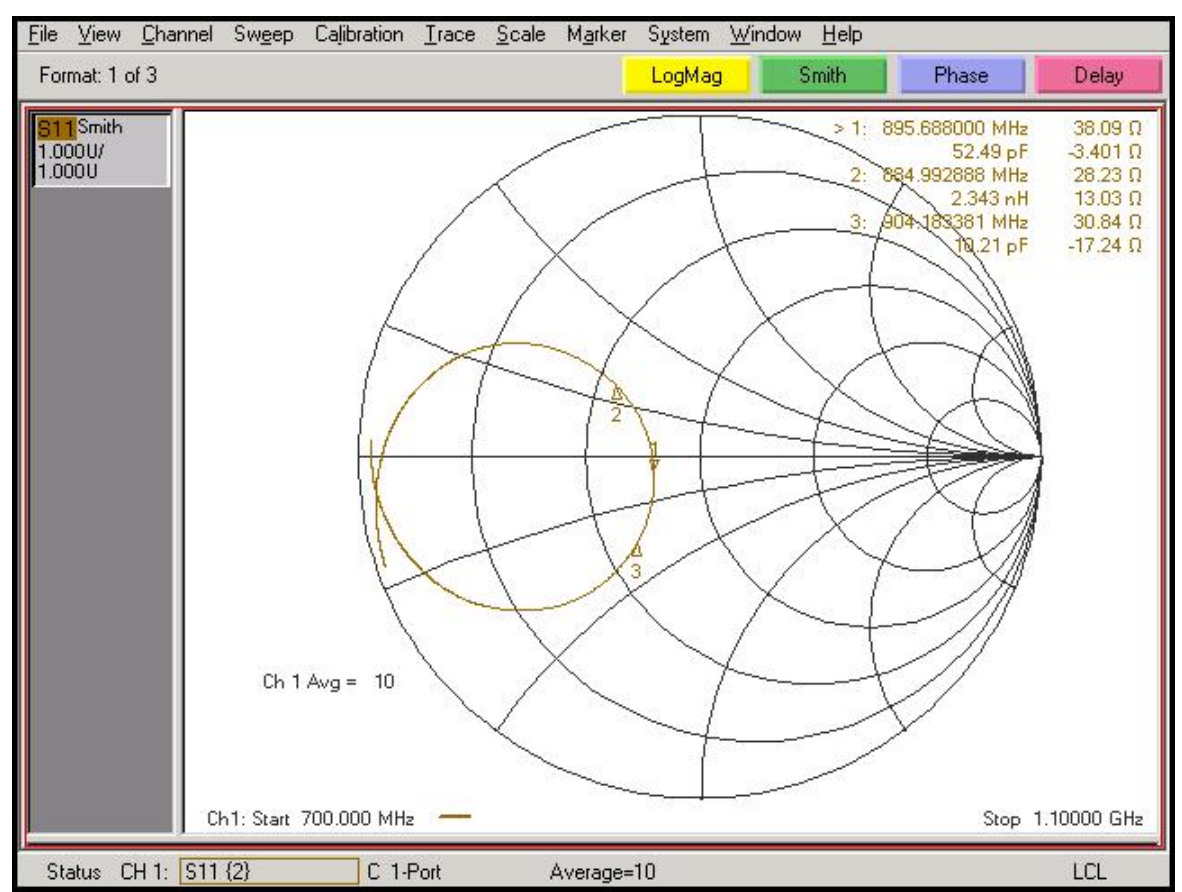

Figure 5.3.6: Short meandering-monopole input-port reflection coefficient (Smith Chart) after addition of a matching network. 


\subsubsection{Voltage Standing Wave Ratio (VSWR)}

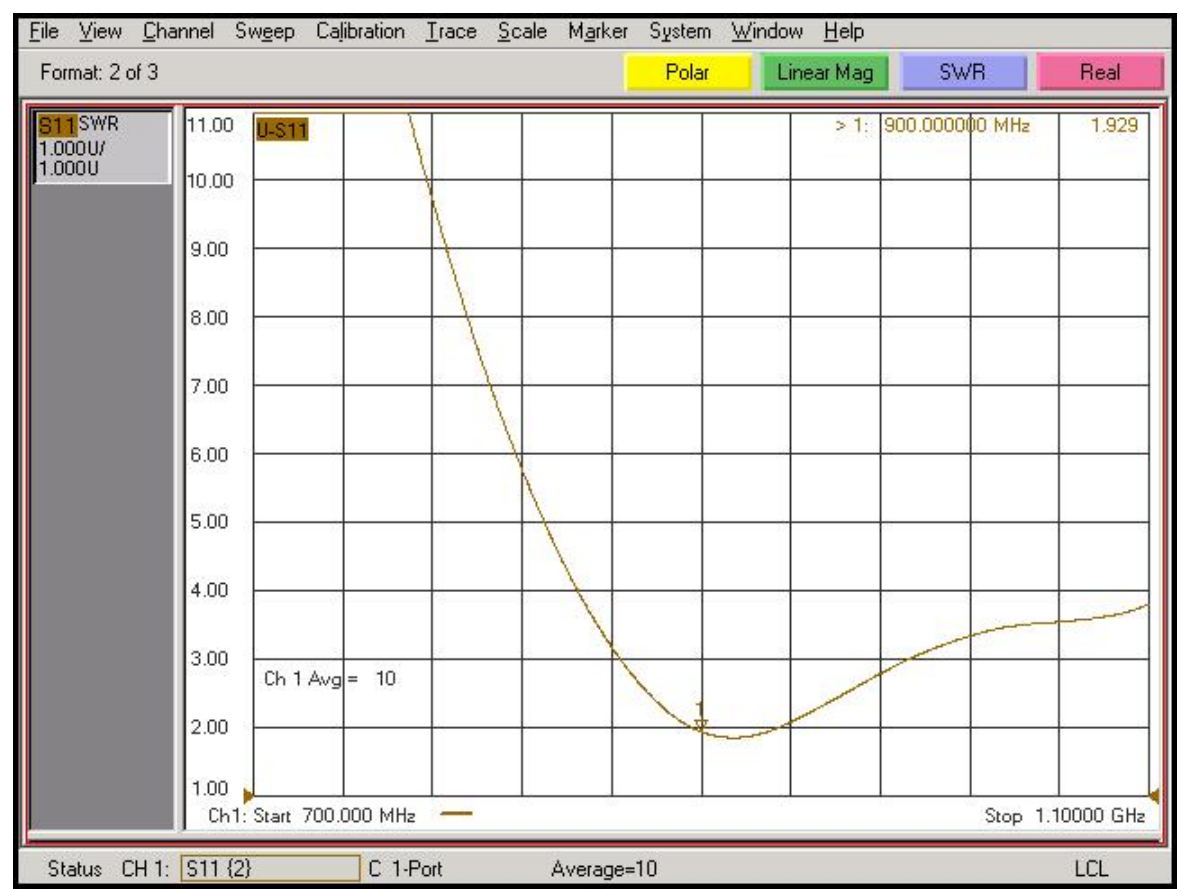

Figure 5.3.7: Short meandering-monopole input-port Voltage Standing Wave Ratio (VSWR) before addition of a matching network.

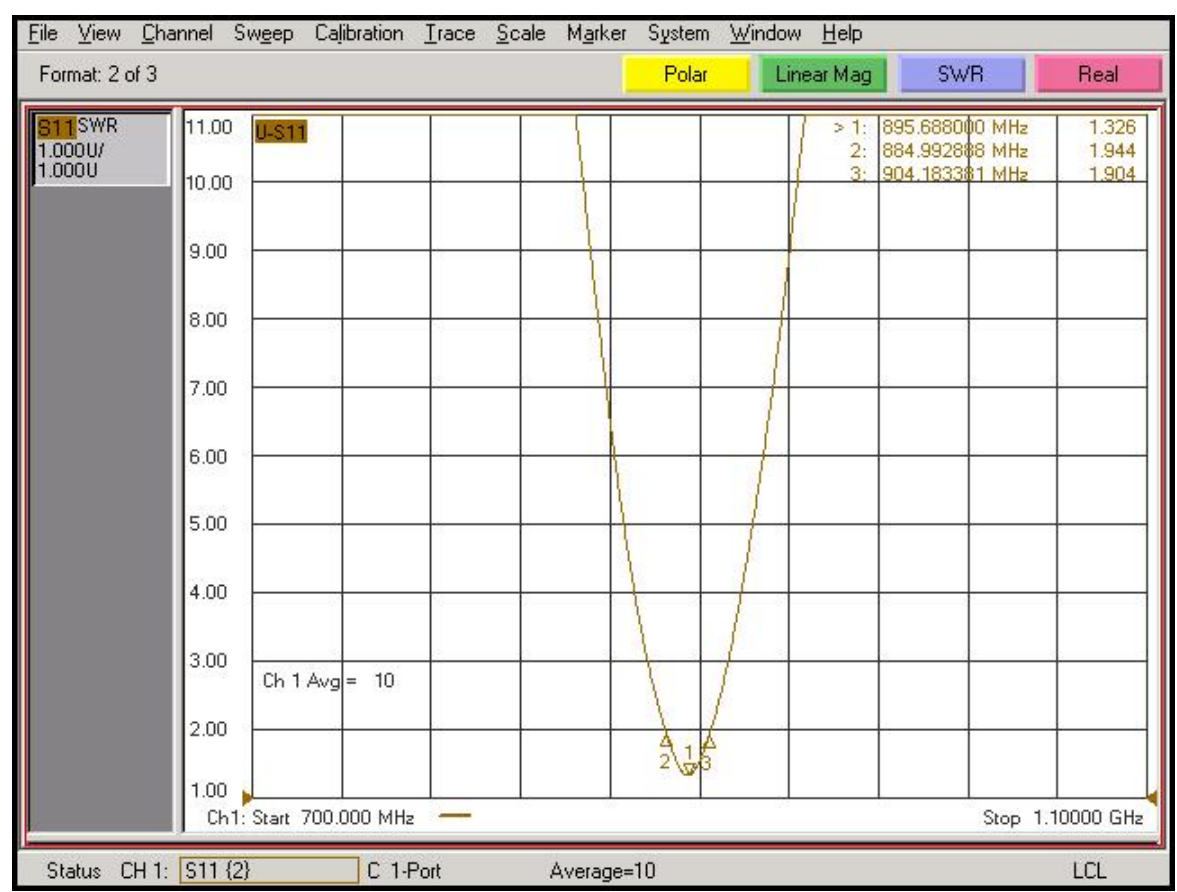

Figure 5.3.8: Short meandering-monopole Input-Port Voltage Standing Wave Ratio (VSWR) after addition of a matching network. 


\subsection{Compact Meandering-Monopole Antenna Results}

\subsubsection{Antenna Orientation and Matching Circuit}

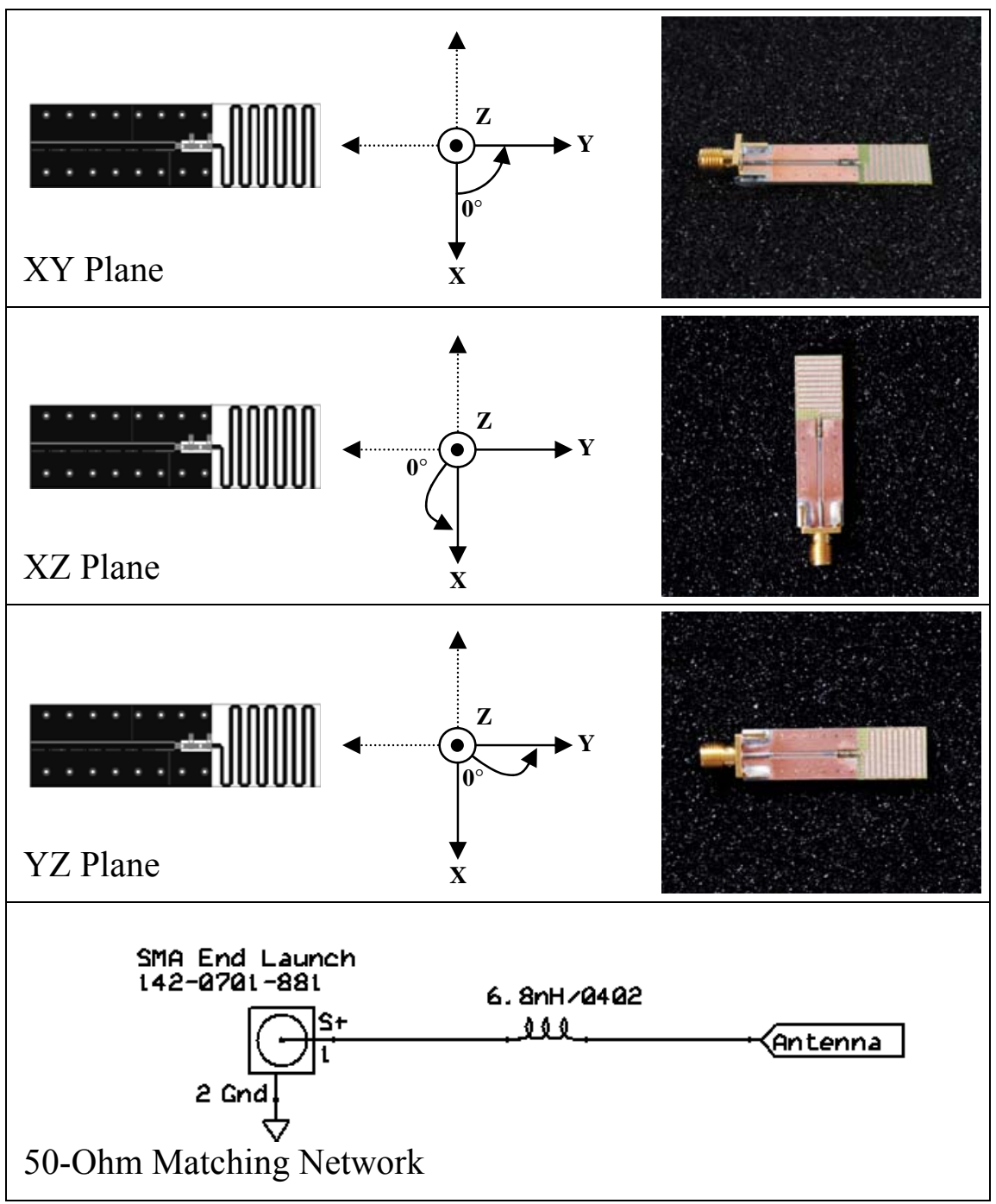

Figure 5.4.1: Compact meandering-monopole orientation and 50-Ohm matching network. 


\subsubsection{D Radiation Pattern after Matching}

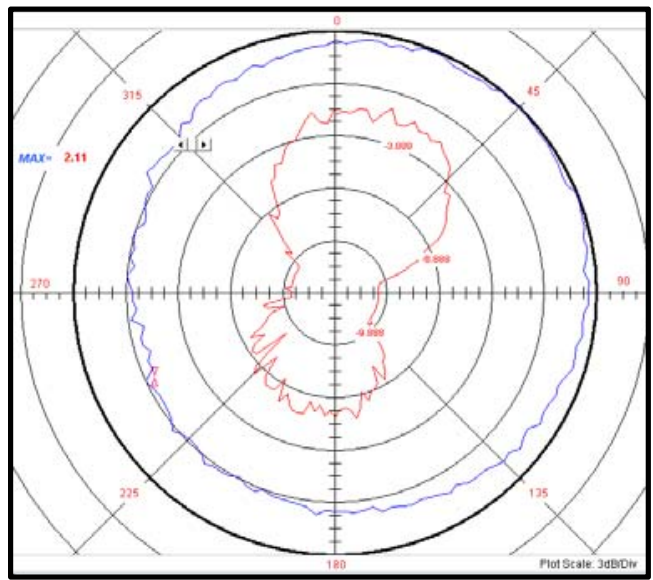

\begin{tabular}{l|ll|}
$\begin{array}{l}\text { Trace: } \mathbf{X Y} \\
\text { Plane }\end{array}$ & $\begin{array}{l}\text { Vertical } \\
\text { Polarization } \\
\text { (Blue) }\end{array}$ & $\begin{array}{l}\text { Horizontal } \\
\text { Polarization } \\
\text { (Red) }\end{array}$ \\
\hline $\begin{array}{l}\text { Frequency } \\
\text { Format }\end{array}$ & $\begin{array}{l}\text { 200 MHz } \\
\text { Azimuth }\end{array}$ & $\mathrm{AZ}=0-360 \mathrm{MHz}$ \\
Zenith & $\mathrm{Z}=90$ & $\mathrm{AZ}=0-360$ \\
Gain Min. & -1.835718 & $\mathrm{Z}=90$ \\
Gain Max. & 2.111581 & -24.714450 \\
Avg. Gain & 0.409187 & -7.200355
\end{tabular}

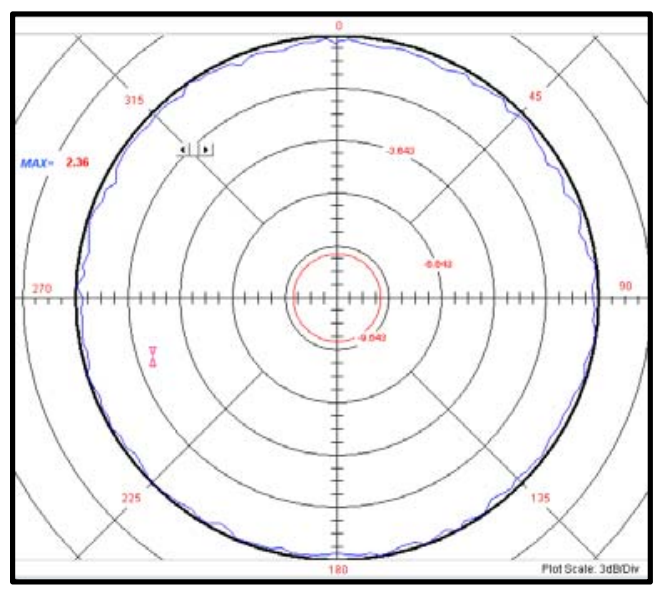

\begin{tabular}{l|ll|}
$\begin{array}{l}\text { Trace: } \mathbf{X Z} \\
\text { Plane }\end{array}$ & $\begin{array}{l}\text { Vertical } \\
\text { Polarization } \\
\text { (Blue) }\end{array}$ & $\begin{array}{l}\text { Horizontal } \\
\text { Polarization } \\
\text { (Red) }\end{array}$ \\
\hline $\begin{array}{l}\text { Frequency } \\
\text { Format }\end{array}$ & $\begin{array}{l}\text { 200 } \mathrm{MHz} \\
900 \mathrm{MHz}\end{array}$ \\
$\begin{array}{l}\text { Azimuth } \\
\text { Zenith }\end{array}$ & $\mathrm{AZ}=0$ & 20Log \\
Gain Min. & 1.612525 & -18.255108 \\
Gain Max. & 2.356678 & -11.859288 \\
Avg. Gain & 2.043632 & -15.053377
\end{tabular}

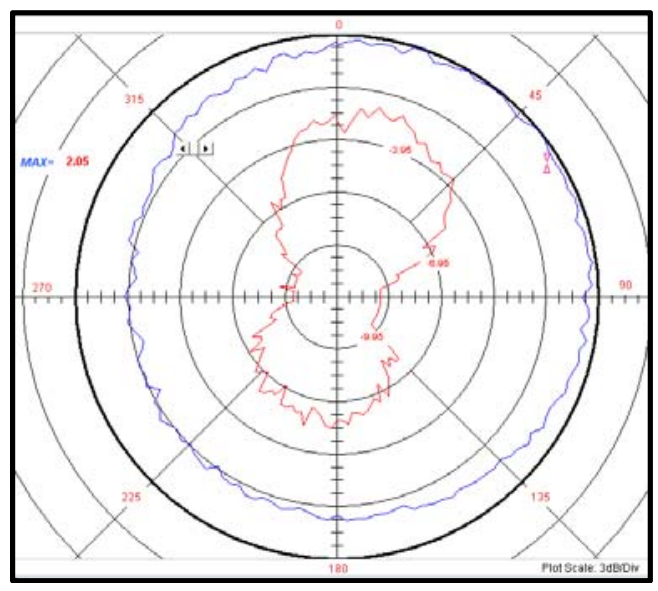

\begin{tabular}{lll|}
$\begin{array}{l}\text { Trace: YZ } \\
\text { Plane }\end{array}$ & $\begin{array}{l}\text { Vertical } \\
\text { Polarization } \\
\text { (Blue) }\end{array}$ & $\begin{array}{l}\text { Horizontal } \\
\text { Polarization } \\
\text { (Red) }\end{array}$ \\
\hline $\begin{array}{l}\text { Frequency } \\
\text { Format }\end{array}$ & $900 \mathrm{MHz}$ & $900 \mathrm{MHz}$ \\
Azimuth & $\mathrm{AZ}=90$ & 20Log \\
Zenith & $\mathrm{Z}=0-360$ & $\mathrm{Z}=0-360$ \\
Gain Min. & -1.582345 & -25.830331 \\
Gain Max. & 2.050275 & -1.842455 \\
Avg. Gain & 0.427233 & -7.206051
\end{tabular}

Figure 5.4.2: Compact meandering-monopole 2D-radiation pattern expressed as gain in $\mathrm{dB}$ for $\mathrm{XY}, \mathrm{XZ}$, and YZ planes after addition of a matching network. 


\subsubsection{S11 Reflection Coefficient (dB)}

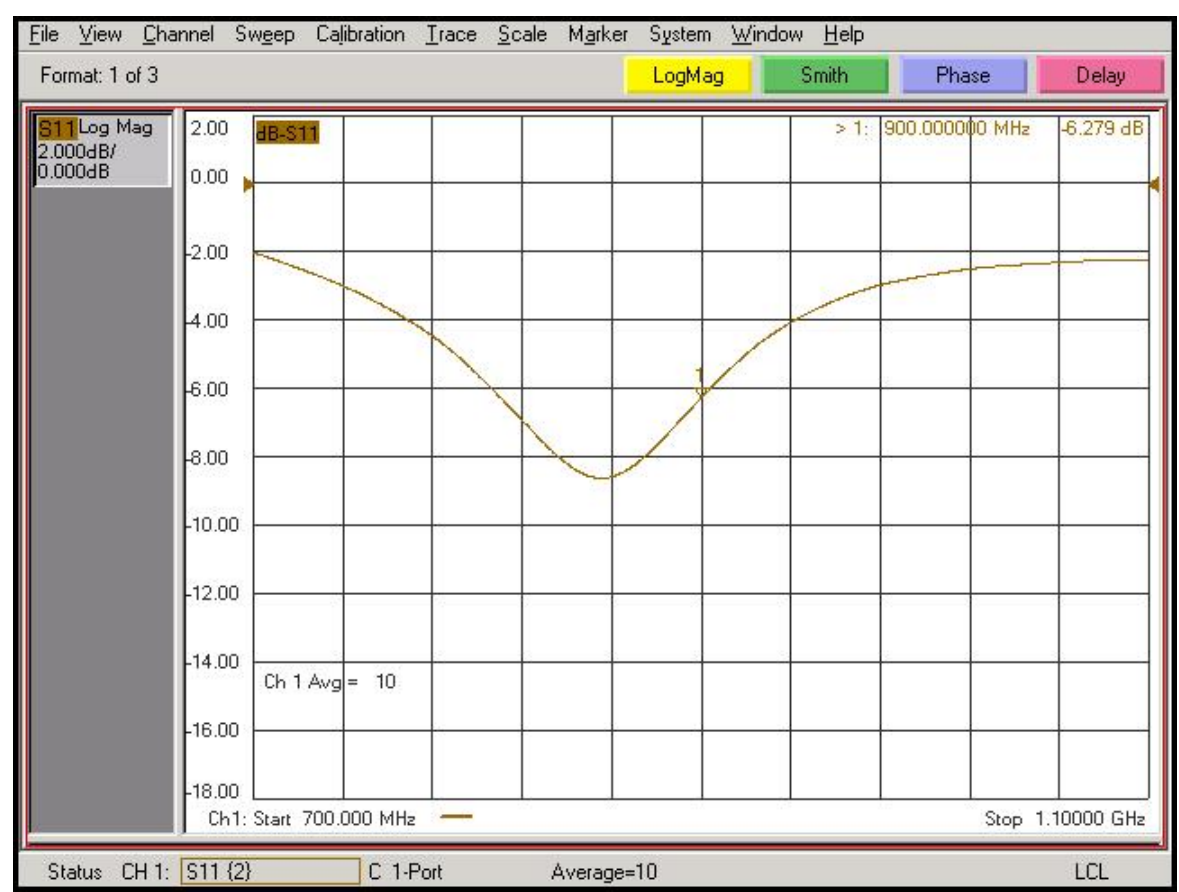

Figure 5.4.3: Compact meandering-monopole input-port reflection coefficient (S11) expressed in $\mathrm{dB}$ before addition of a matching network.

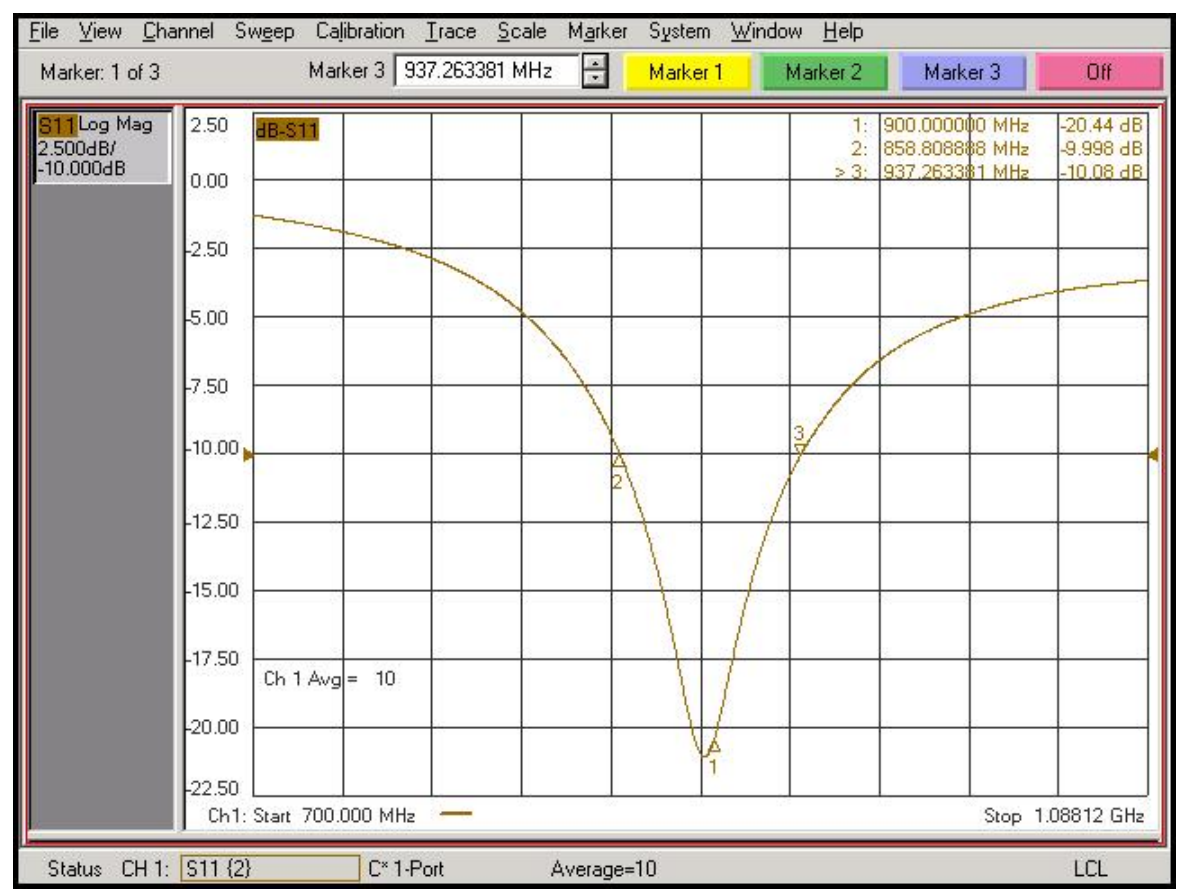

Figure 5.4.4: Compact meandering-monopole input-port reflection coefficient (S11) expressed in $\mathrm{dB}$ after addition of a matching network. 


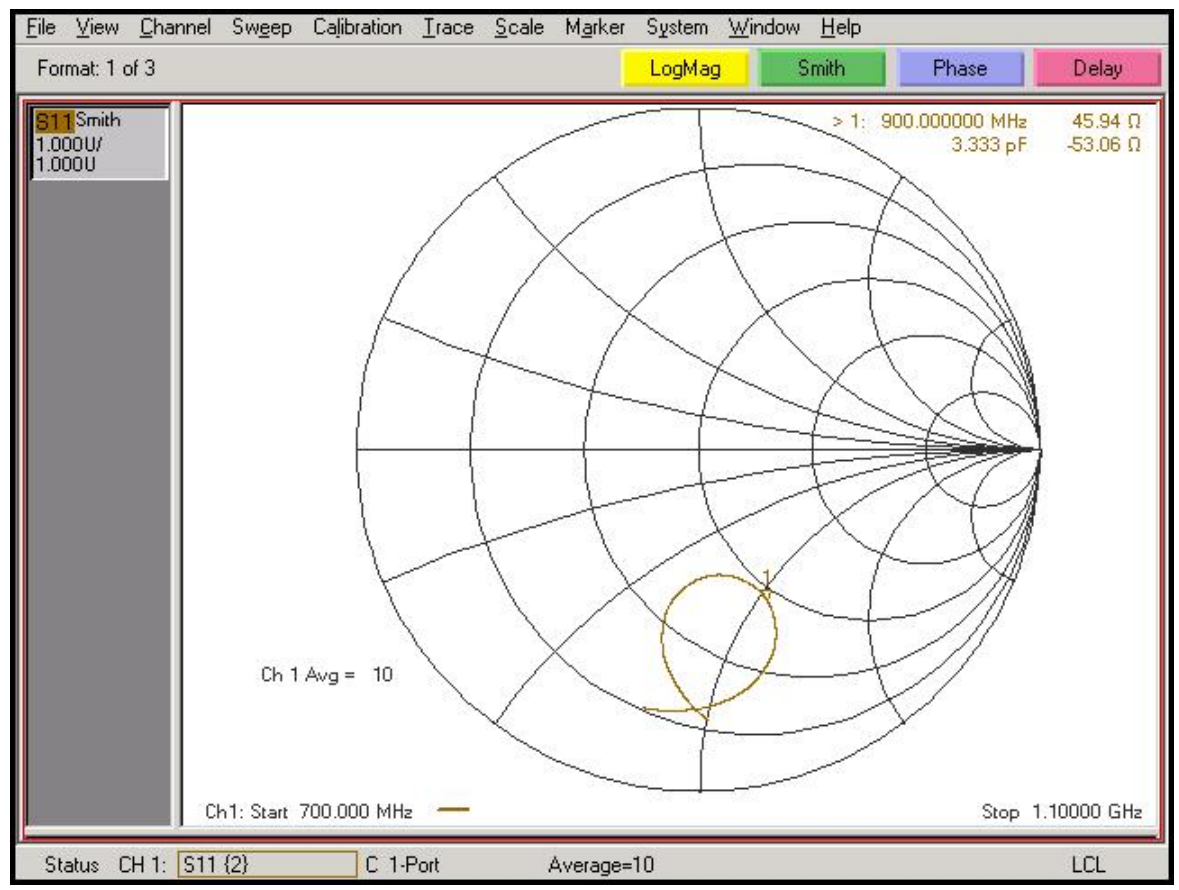

Figure 5.4.5: Compact meandering-monopole input-port reflection coefficient (Smith Chart) before addition of a matching network.

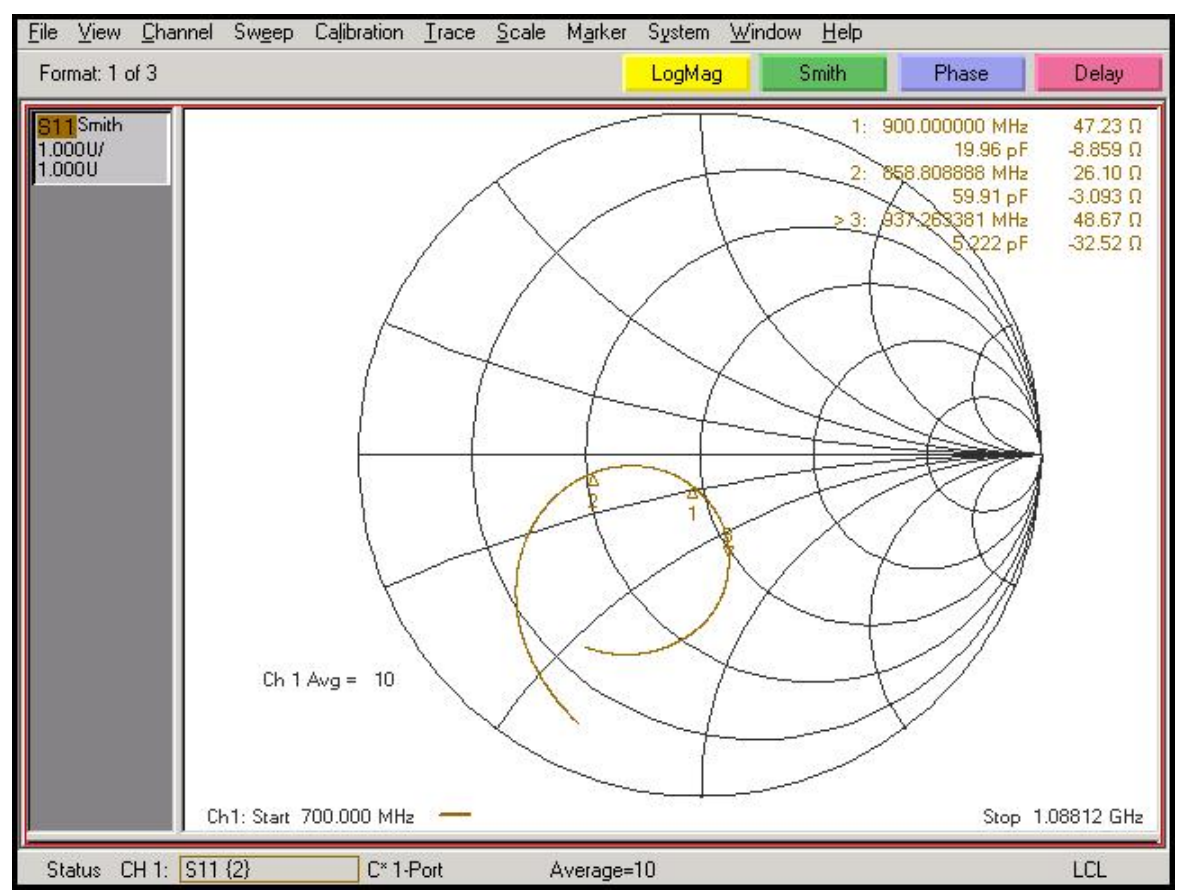

Figure 5.4.6: Compact meandering-monopole input-port reflection coefficient (Smith Chart) after addition of a matching network. 


\subsubsection{Voltage Standing Wave Ratio (VSWR)}

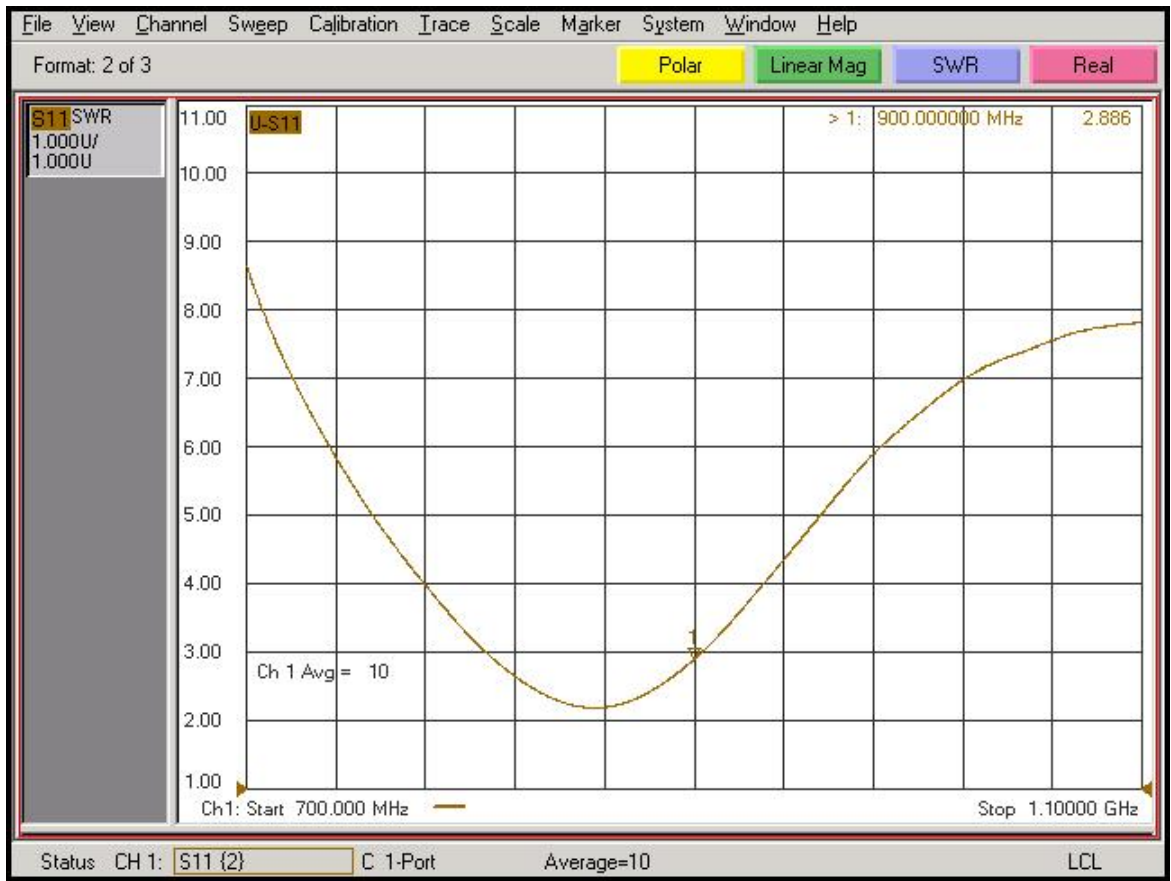

Figure 5.4.7: Compact meandering-monopole input-port Voltage Standing Wave Ratio (VSWR) before addition of a matching network.

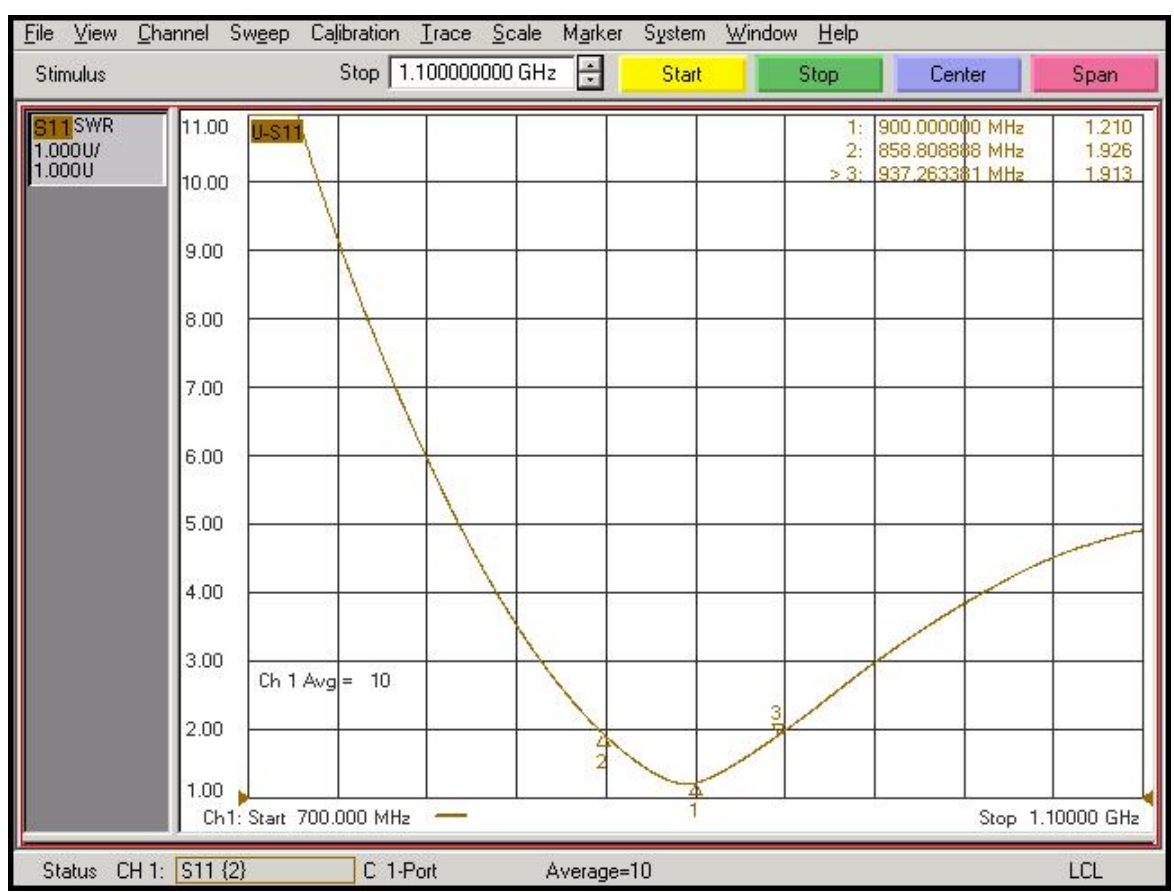

Figure 5.4.8: Compact meandering-monopole input-port Voltage Standing Wave Ratio (VSWR) after addition of a matching network. 


\section{$5.5 \quad$ Loop Antenna Results}

\subsubsection{Antenna Orientation and Matching Circuit}

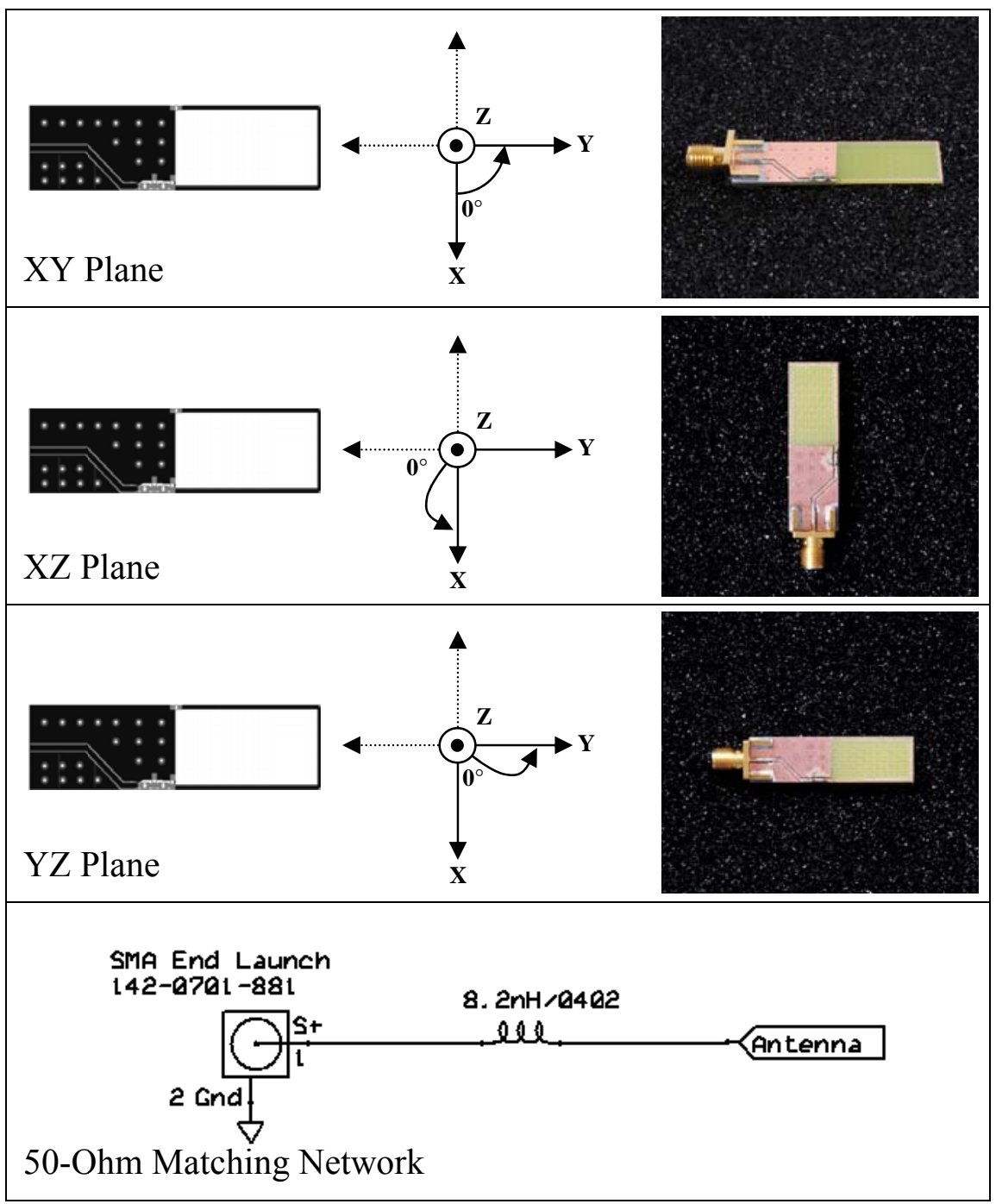

Figure 5.5.1: Compact meandering-monopole orientation and 50-Ohm matching network. 


\subsubsection{D Radiation Pattern after Matching}

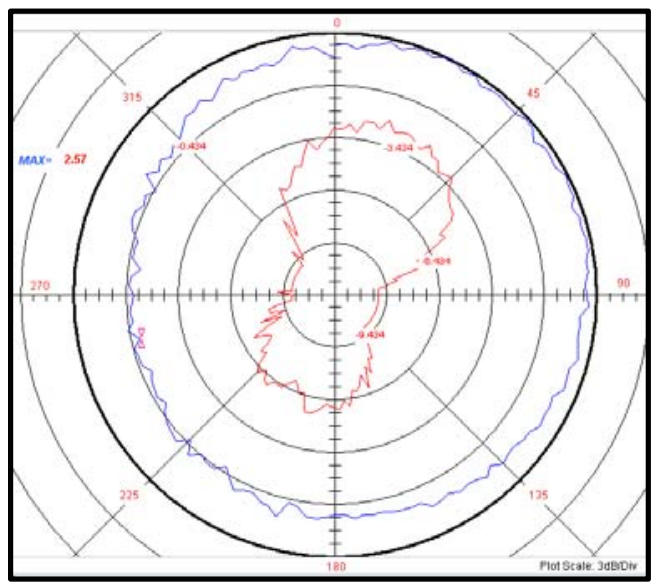

\begin{tabular}{l|ll|}
$\begin{array}{l}\text { Trace: } \mathbf{X Y} \\
\text { Plane }\end{array}$ & $\begin{array}{l}\text { Vertical } \\
\text { Polarization } \\
\text { (Blue) }\end{array}$ & $\begin{array}{l}\text { Horizontal } \\
\text { Polarization } \\
\text { (Red) }\end{array}$ \\
\hline $\begin{array}{l}\text { Frequency } \\
\text { Format }\end{array}$ & $\begin{array}{l}200 \mathrm{MHz} \\
900 \mathrm{MHz}\end{array}$ \\
Azimuth & $\mathrm{AZ}=0-360$ & $\mathrm{AZ}=0-360$ \\
Zenith & $\mathrm{Z}=90$ & $\mathrm{Z}=90$ \\
Gain Min. & -1.208675 & -22.850369 \\
Gain Max. & 2.566039 & -1.978125 \\
Avg. Gain & 0.901678 & -7.358592
\end{tabular}

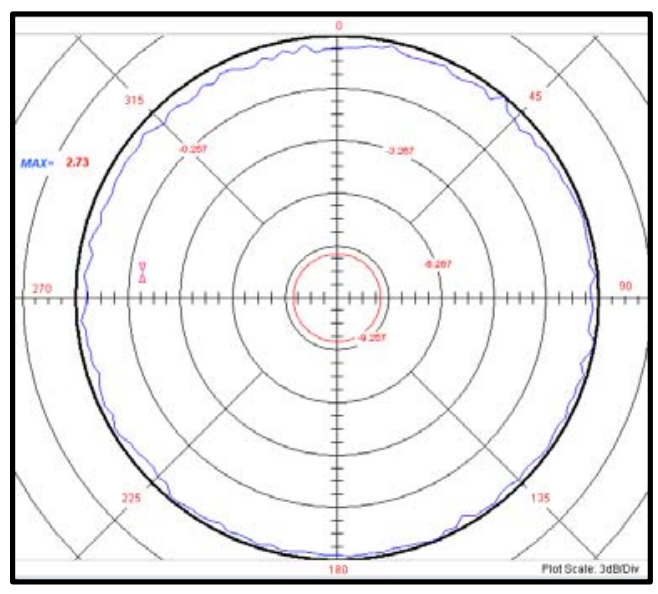

\begin{tabular}{|c|c|c|}
\hline $\begin{array}{l}\text { Trace: XZ } \\
\text { Plane }\end{array}$ & $\begin{array}{l}\text { Vertical } \\
\text { Polarization } \\
\text { (Blue) }\end{array}$ & $\begin{array}{l}\text { Horizontal } \\
\text { Polarization } \\
\text { (Red) }\end{array}$ \\
\hline Frequency & $900 \mathrm{MHz}$ & $900 \mathrm{MHz}$ \\
\hline Format & 20Log & 20Log \\
\hline Azimuth & $A Z=0$ & $A Z=0$ \\
\hline Zenith & $Z=0-360$ & $Z=0-360$ \\
\hline Gain Min. & 1.771435 & -20.181991 \\
\hline Gain Max. & 2.733039 & -14.029143 \\
\hline Avg. Gain & 2.255082 & -16.537740 \\
\hline
\end{tabular}

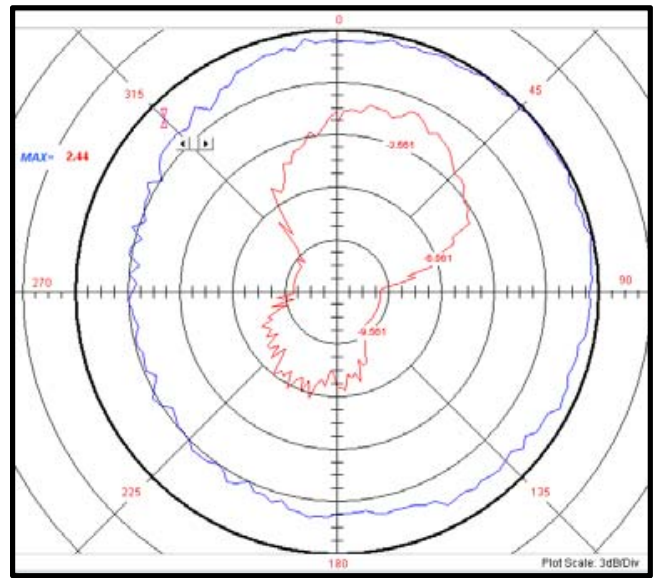

\begin{tabular}{lll|}
$\begin{array}{l}\text { Trace: YZ } \\
\text { Plane }\end{array}$ & $\begin{array}{l}\text { Vertical } \\
\text { Polarization } \\
\text { (Blue) }\end{array}$ & $\begin{array}{l}\text { Horizontal } \\
\text { Polarization } \\
\text { (Red) }\end{array}$ \\
\hline $\begin{array}{l}\text { Frequency } \\
\text { Format }\end{array}$ & $900 \mathrm{MHz}$ & $900 \mathrm{MHz}$ \\
Azimuth & $\mathrm{AZ}=90$ & AZ \\
Zenith & $\mathrm{Z}=0-360$ & $\mathrm{Z}=0-360$ \\
Gain Min. & -1.169779 & -24.238479 \\
Gain Max. & 2.439339 & -1.417685 \\
Avg. Gain & 0.859264 & -7.257388
\end{tabular}

Figure 5.5.2: Loop antenna 2D-radiation pattern expressed as gain in $\mathrm{dB}$ for $\mathrm{XY}$, $\mathrm{XZ}$, and YZ planes after addition of a matching network. 


\subsubsection{S11 Reflection Coefficient (dB)}

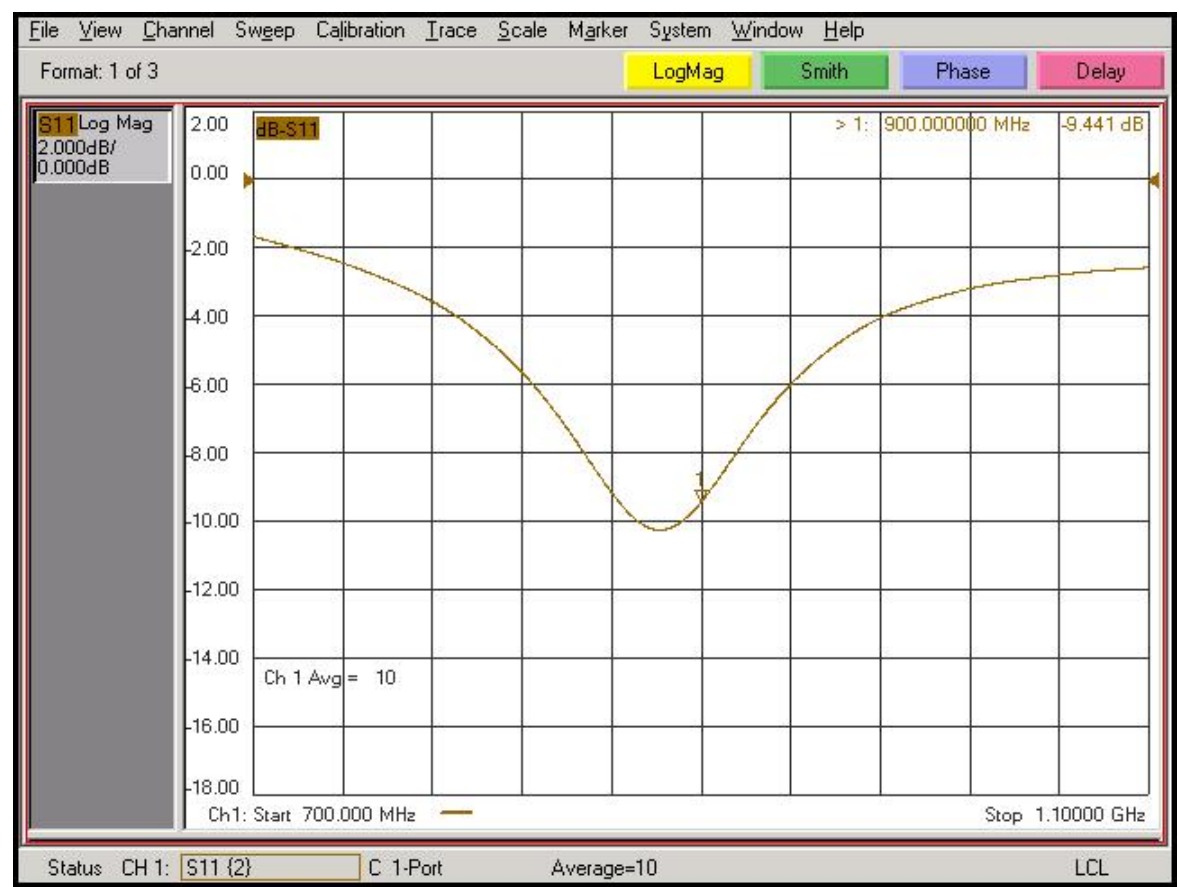

Figure 5.5.3: Loop antenna input-port reflection coefficient (S11) expressed in dB before addition of a matching network.

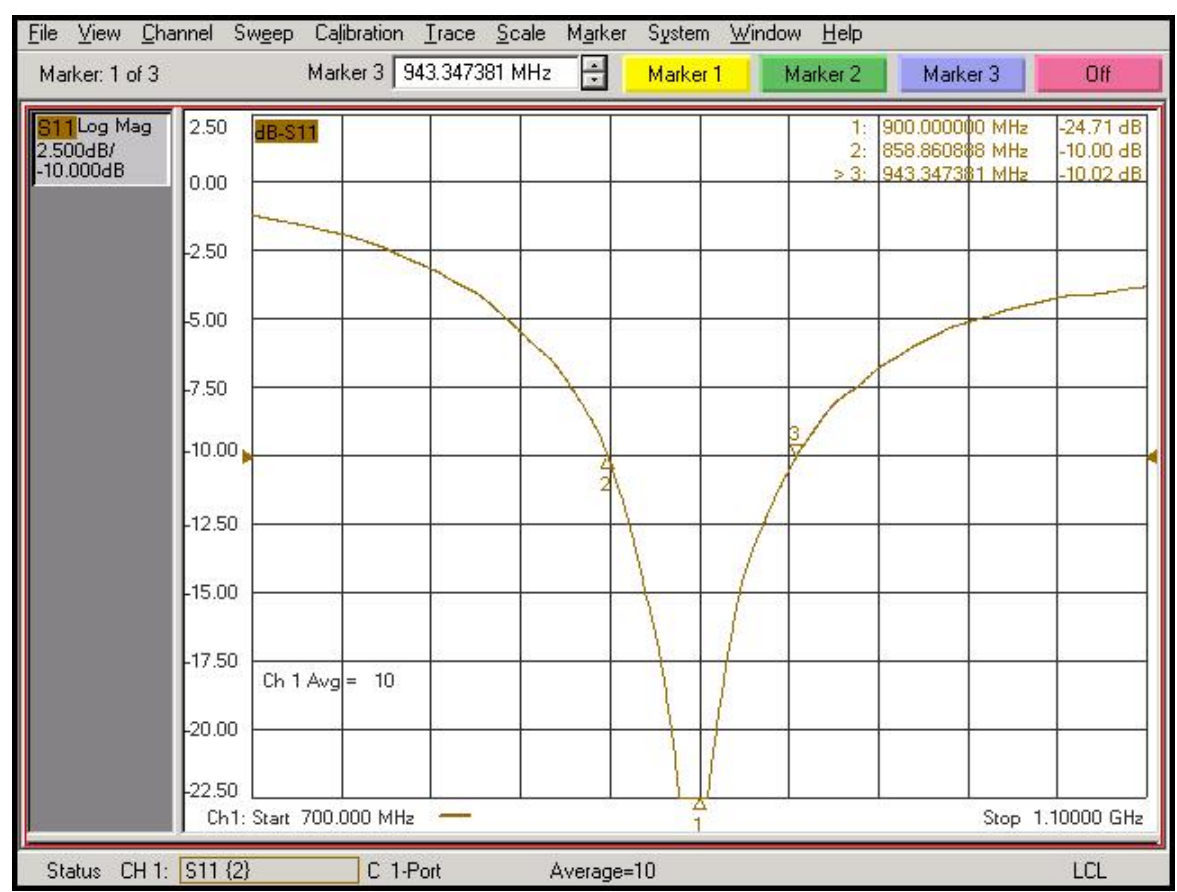

Figure 5.5.4: Loop antenna input-port reflection coefficient (S11) expressed in dB after addition of a matching network. 


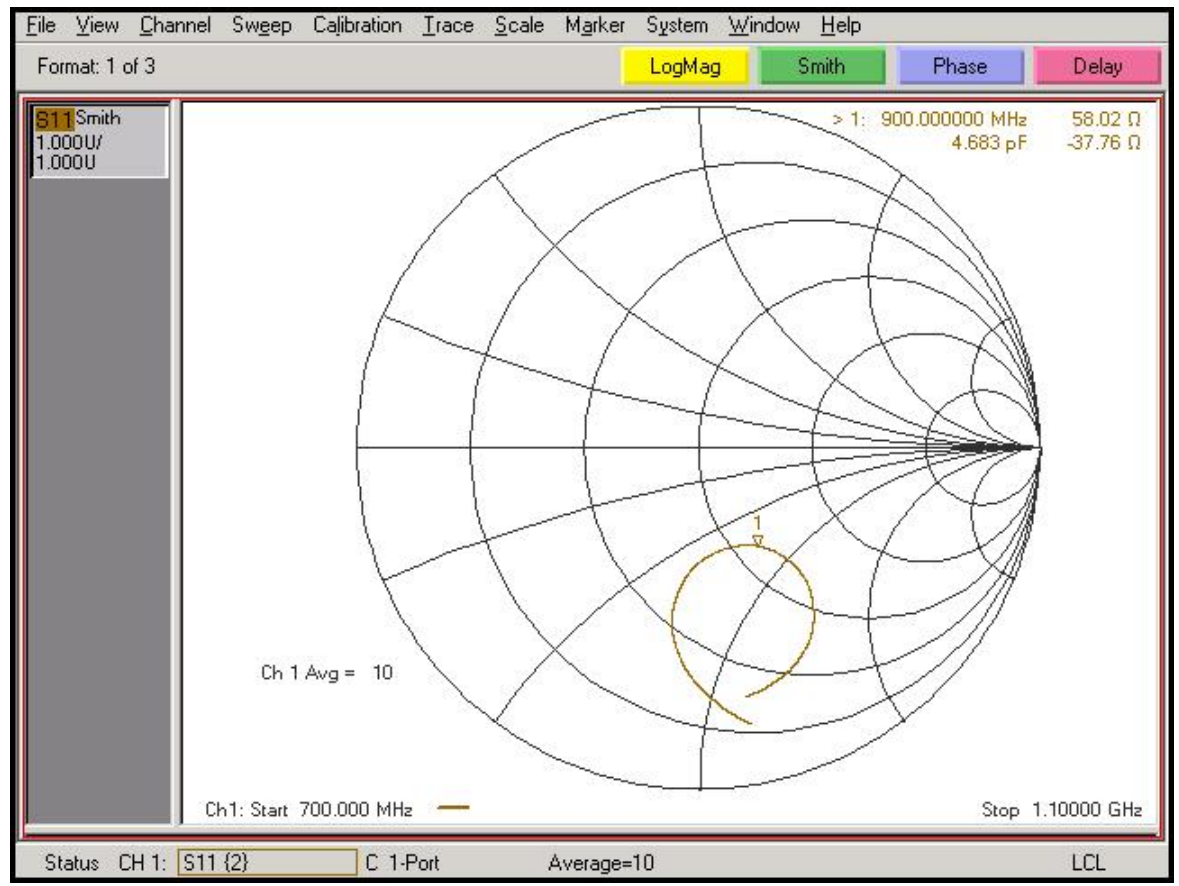

Figure 5.5.5: Loop antenna input-port reflection coefficient (Smith Chart) before addition of a matching network.

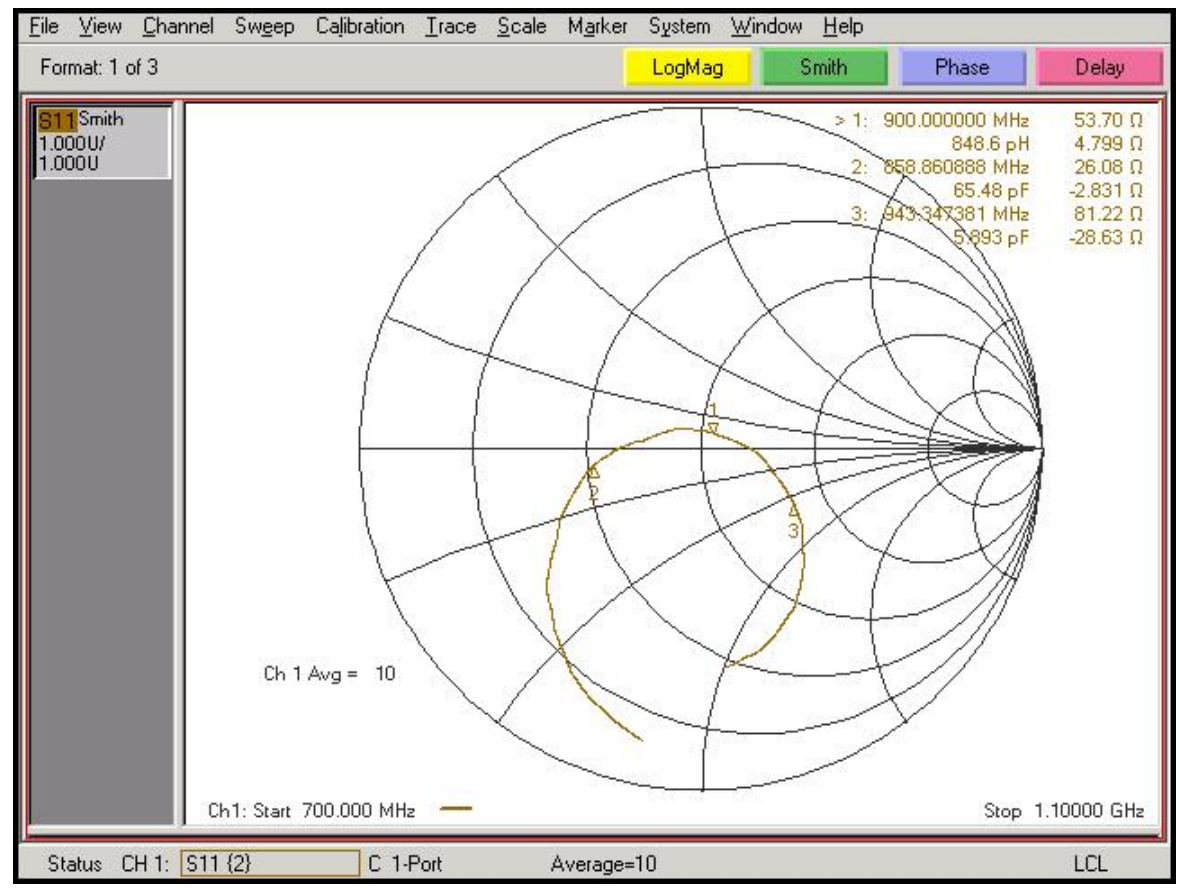

Figure 5.5.6: Loop antenna input-port reflection coefficient (Smith Chart) after addition of a matching network. 


\subsubsection{Voltage Standing Wave Ratio (VSWR)}

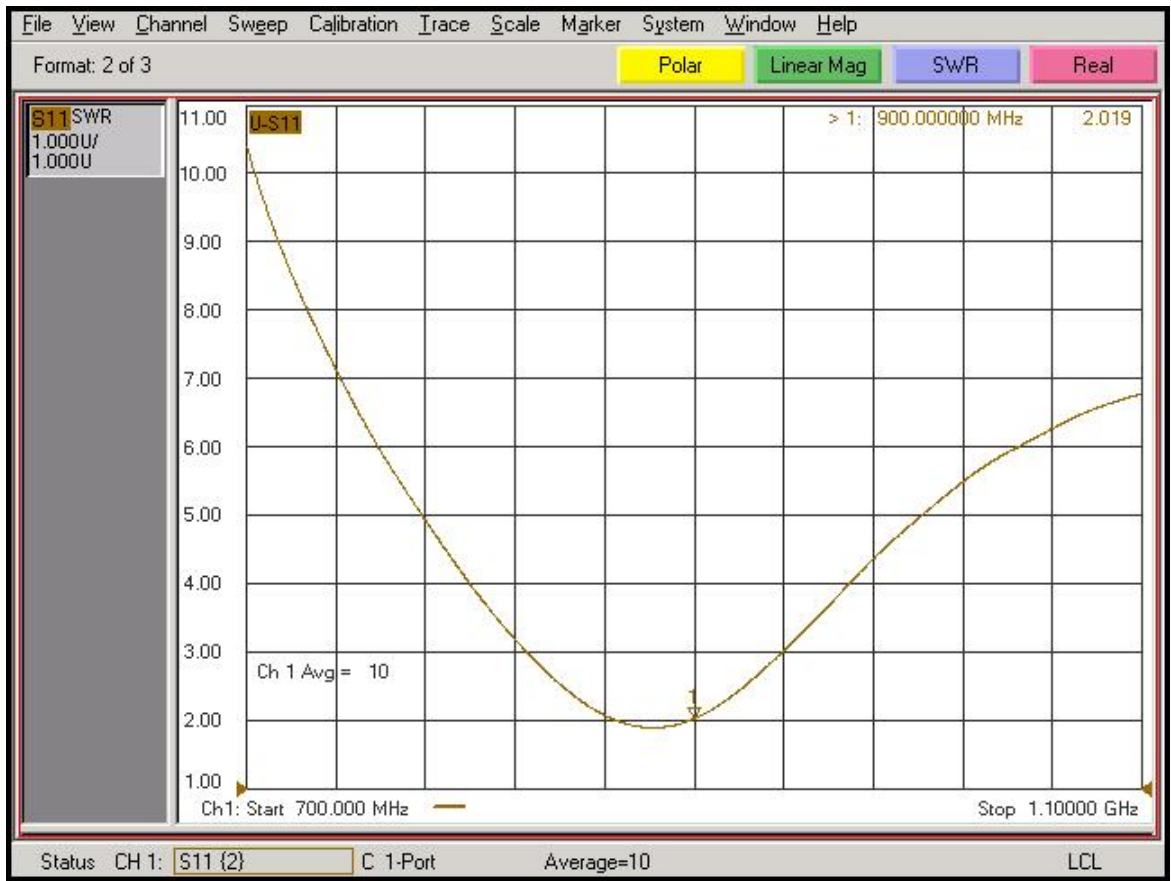

Figure 5.5.7: Loop antenna input-port Voltage Standing Wave Ratio (VSWR) before addition of a matching network.

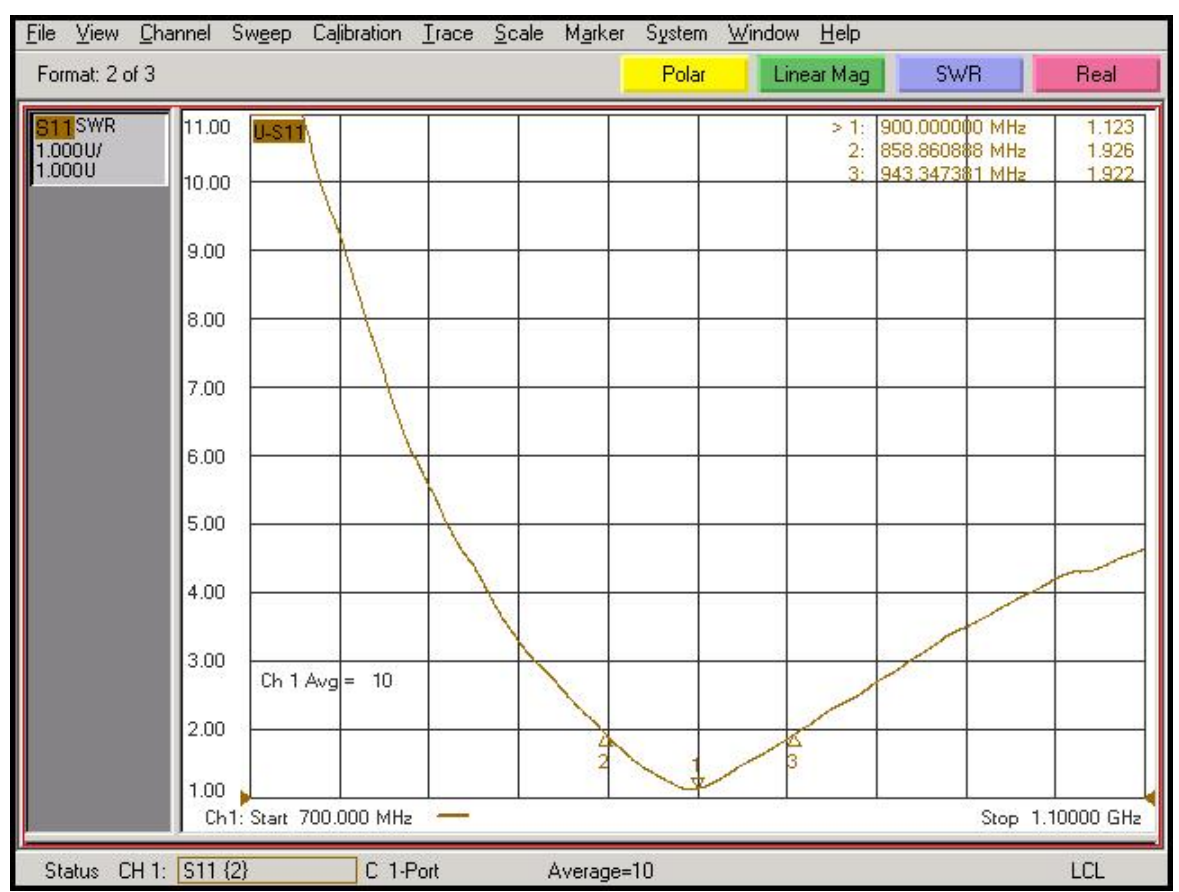

Figure 5.5.8: Loop antenna input-port Voltage Standing Wave Ratio (VSWR) after addition of a matching network. 


\section{Integrated Transceiver Testing}

\subsection{Portable Range Testing PCBs}

The portable range testing PCBs contains an SiLabs c8051F340 microprocessor to control a CC1100 Chipcon transceiver over 3-wire SPI bus. The tool kit contains an onboard power supply, LCD display, UART level shifter, USB interface, pushbuttons, indication LEDs, and transceiver circuitry. It is highly configurable and easily modified to allow for the fine tuning of matching circuitry.

The main purpose of the prototype development tool kit is to facilitate a wide variety of tests. Its main goal is to demonstrate round trip communication while displaying Received Signal Strength Indicator (RSSI) information. A pair of battery powered portable range testing PCBs can be used to measure signal strength verse range for the AUT. The pushbutton interface allows easy on-the-fly changes for output power, data rate, frequency, modulation format, and filter bandwidths. This battery powered tool is used for field testing to discover the maximum attainable distance for reliable round-trip communications. Two range testing prototype development tool kits are shown below in Figure 6.1.1.

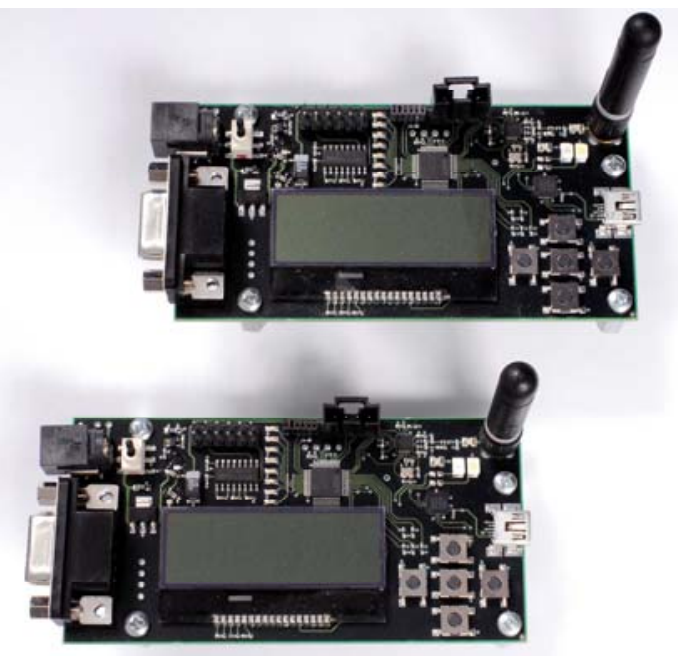

Figure 6.1.1: Two prototype development tool kit PCBs. 


\subsection{CC1100 Typical Application}

The radio portion of the portable range testing PCB design uses a Chipcon CC1100 transceiver. The reference circuit Bill of Materials (BOM) and design schematic is shown below in Table 6.4-1 and Figure 6.2.1.

Table 6.2-1: Reference Circuit Bill Of Materials (9).

\begin{tabular}{|lll|}
\hline Component & Value at 868/915MHz & Manufacturer \\
\hline C51 & $100 \mathrm{nF} \pm 10 \%, 0402 \times 5 R$ & Murata GRM1555C series \\
C81 & $27 \mathrm{pF} \pm 5 \%, 0402 \mathrm{NPO}$ & Murata GRM1555C series \\
C101 & $27 \mathrm{pF} \pm 5 \%, 0402 \mathrm{NPO}$ & Murata GRM1555C series \\
C121 & $1.0 \mathrm{pF} \pm 0.25 \mathrm{pF}, 0402 \mathrm{NP0}$ & Murata GRM1555C series \\
C122 & $1.5 \mathrm{pF} \pm 0.25 \mathrm{pF}, 0402 \mathrm{NPO}$ & Murata GRM1555C series \\
C123 & $3.3 \mathrm{pF} \pm 0.25 \mathrm{pF}, 0402 \mathrm{NPO}$ & Murata GRM1555C series \\
C124 & $100 \mathrm{pF} \pm 5 \%, 0402 \mathrm{NPO}$ & Murata GRM1555C series \\
C125 & $100 \mathrm{pF} \pm 5 \%, 0402 \mathrm{NPO}$ & Murata GRM1555C series \\
C131 & $1.5 \mathrm{pF} \pm 0.25 \mathrm{pF}, 0402 \mathrm{NPO}$ & Murata GRM1555C series \\
L121 & $12 \mathrm{nH} \pm 5 \%, 0402$ monolithic & Murata LQG15HS series \\
L122 & $18 \mathrm{nH} \pm 5 \%, 0402$ monolithic & Murata LQG15HS series \\
L123 & $12 \mathrm{nH} \pm 5 \%, 0402$ monolithic & Murata LQG15HS series \\
L124 & $12 \mathrm{nH} \pm 5 \%, 0402$ monolithic & Murata LQG15HS series \\
L131 & $12 \mathrm{nH} \pm 5 \%, 0402$ monolithic & Murata LQG15HS series \\
L132 & $18 \mathrm{nH} \pm 5 \%, 0402$ monolithic & Murata LQG15HS series \\
R171 & $56 \mathrm{k} \Omega \pm 1 \%, 0402$ & Koa RK73 series \\
XTAL & $26.0 \mathrm{MHz}$ surface mount crystal & NDK, AT-41CD2 \\
\hline
\end{tabular}

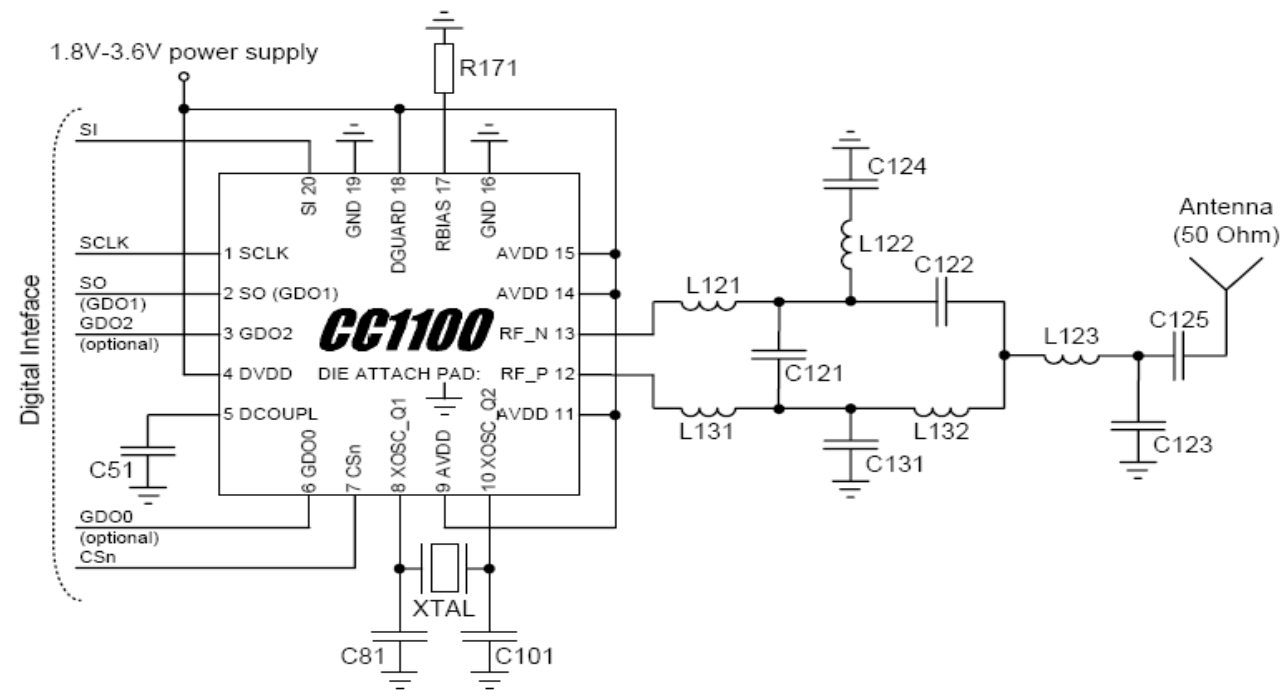

Figure 6.2.1: Reference circuit schematic provided by Chipcon (9). 


\subsection{Balun Matching Network}

A balanced-to-unbalanced matching network is required to optimize power transfer from the CC1100 transceiver to the antenna load. A balun is used to convert transceiver differential output impedance to an easily matched single-ended 50-Ohm output. For small-signal RF circuits, power to the load is maximized using conjugatematching of the expected 50-ohm antenna load impedance to the transmitter source impedance. Because the transceiver output impedance has an 86.5 - j43 Ohm output impedance, load impedance as seen looking in by the transceiver must be exactly 86.5 $+\mathrm{j} 43 \mathrm{Ohms}$ to maximize the power delivered to the antenna. This overall impedance includes the impedance of the balun, matching circuit, and antenna.

The output of the balun matching circuitry is optimized for a 50-Ohm antenna load. Consequently, all antennas have been designed to accommodate 50-Ohm input impedance. Each antenna includes its own additional set of matching components allowing it to be matched to $50 \mathrm{Ohms}$. This configuration allows any 50 -ohm antenna to be used with the range testing PCBs and provides the ability to exchange antennas on the fly. For an integrated transceiver design with limited space, at least two matching components can be eliminated by simply matching the antenna directly to the single-ended balun output. This space saving effort eliminates the flexibility of testing by attaching an external known 50-Ohm antenna. However, the reduction in overall size and improved efficiency can be beneficial in some designs. 


\subsection{Range Testing}

Range testing is performed using the loop antenna because preliminary results show this configuration is the best performing design. Two battery powered range testing PCBs capable of transmitting $+10 \mathrm{dBm}$ are placed in "Ping-Echo" mode. Two loop antennas are used, one on the pinging device and another on the echoing device. The "pinging" range test PCB is placed on a portable cart while the "echoing" range test PCB is located in a fixed position. Range performance is measured over a 225foot distance using the CC1100's internal signal strength indication for received messages. Received message strength is recorded for the entire round-trip communication as data is transmitted and received. This is accomplished by using the pinging device to continuously interrogate nearby units, repeatedly sending "Ping" data packets every $100 \mathrm{mS}$. When the echoing device receives a ping message, it stores the signal strength indication as the Transmitted RSSI (TxRSSI) value. This TxRSSI value is processed and appended to the "Echo" data packet message to be retransmitted back to the pinging device. When the echoed message is received back at the pining device it stores the signal strength indication as Received RSSI (RxRSSI). At this point, both TxRSSI and RxRSSI values are available for transmission to a laptop with custom data logging software.

Three polarizations of the loop antenna design are measured for range performance, these are denoted as vertical-vertical (VV), vertical-horizontal (VH), and horizontal-horizontal $(\mathrm{HH})$ polarization and shown in Figure 6.4.1 below.

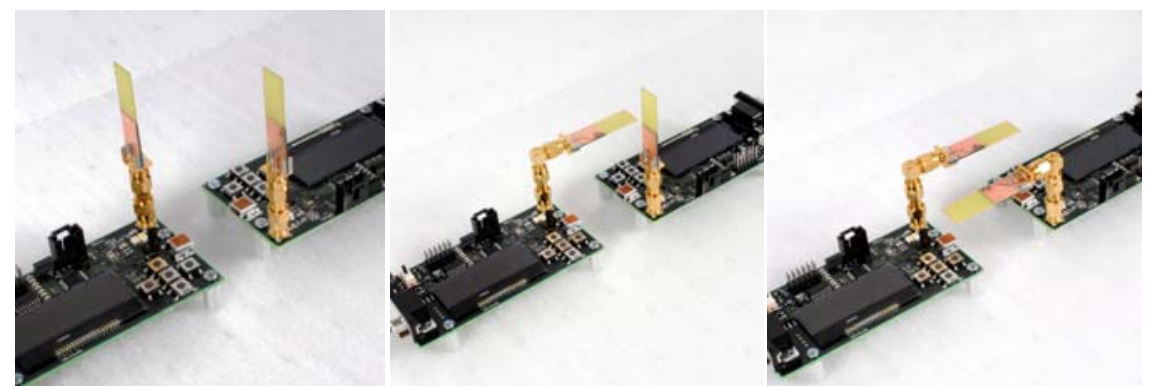

Figure 6.4.1: Loop antenna VV, VH, and HH polarization. 
Additionally in VV polarization, range testing is performed using Face-toFace, Side-to-Side, and Back-to-Back antenna orientations. The positions shown in Figure 6.4.2 are tested to ensure antenna performance is orientation independent.
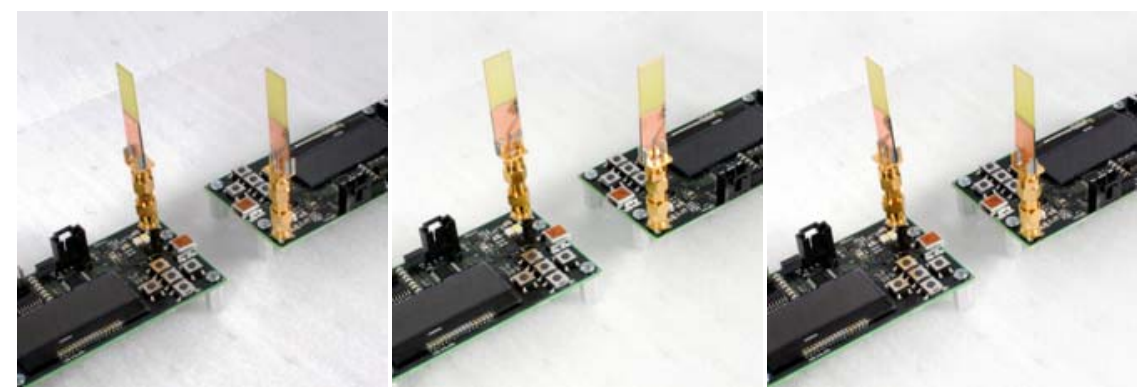

Figure 6.4.2: Face-to-Face, Side-to-Side, and Back-to-Back orientations in VV polarization.

A reference monopole is has been used to provide additional data for comparison with the loop antenna. The reference monopole data can be used to determine the receiver's maximum detectable signal before saturating. An HP Agilent adjustable attenuator is used to find the receiver's Minimum Detectable Signal (MDS). This attenuator is also used to determine the amount of link-budget margin that exists at the maximum 225-foot distance. The reference monopole and HP Agilent adjustable attenuator are both shown below in Figure 6.4.3.
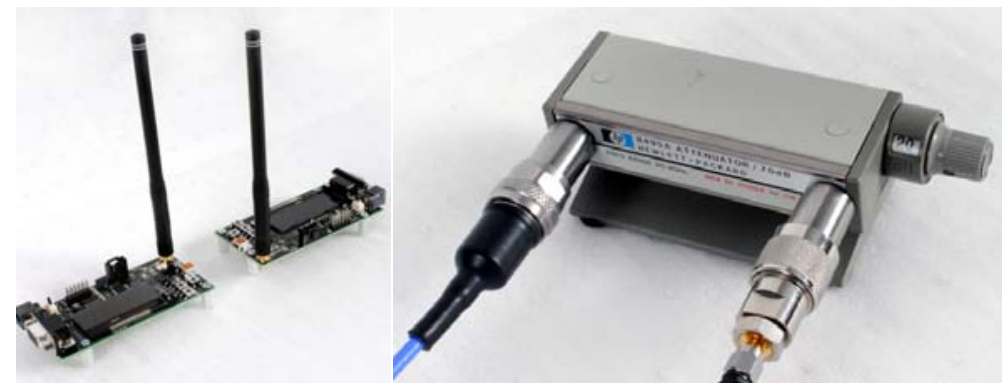

Figure 6.4.3: Reference monopole and HP Agilent 20-dB adjustable attenuator. 
The results for each antenna orientation in VV polarization are presented in Figure 6.4.4. It can be seen each orientation performs with similar results: at the closest range the signal strength is $-40 \mathrm{dBm}$ while at 225 feet the signal strength is approximately $-76 \mathrm{dBm}$. The fact that each orientation performs with similar results is very positive and encouraging.

An additional data set is shown in pink for the Face-to-Face antenna orientation using a $20-\mathrm{dB}$ attenuator. These attenuated loop antenna measurements illustrate functionality at a 225-foot range with an operational margin of at least 20$\mathrm{dB}$. The observed data shows reliable communications, but at this range a $10 \%$ message loss and $-100-\mathrm{dBm}$ signal strength are observed. At this point, message loss is a good indication the receivers minimum detectable signal limit is about to be reached.

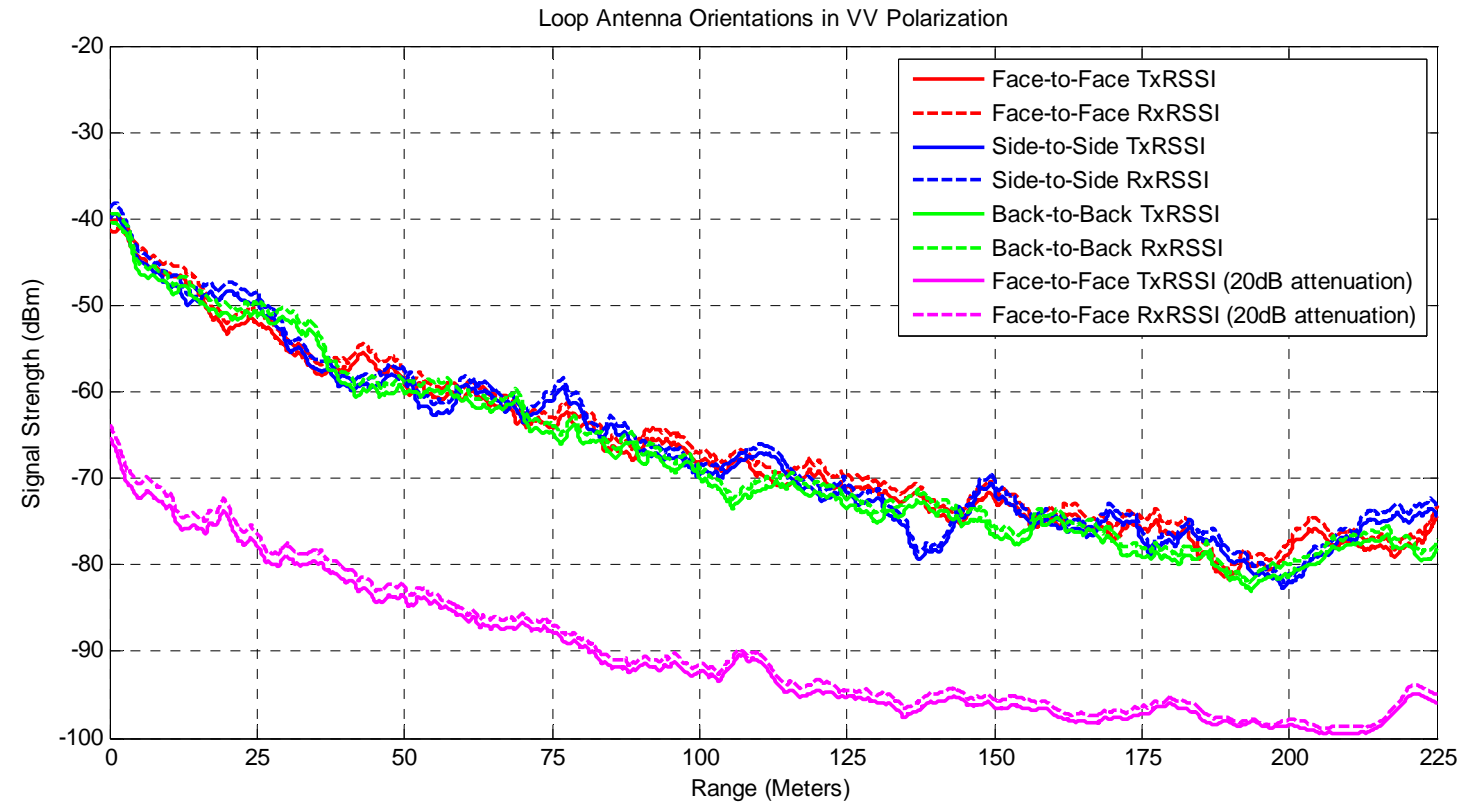

Figure 6.4.4: Plot of signal strength vs range for differing loop antenna orientation in VV polarization. 
The results presented in Figure 6.4.5 show signal strength measurements for the loop antenna $\mathrm{VH}$ and $\mathrm{HH}$ polarizations. Two additional data sets are plotted showing the reference monopole and $20-\mathrm{dB}$ attenuated loop measurements. Observed data shows loop antenna $\mathrm{VH}$ and $\mathrm{HH}$ polarization data very closely resembles loop antenna VV polarization data presented in Figure 6.4.4. The reference monopole is used at very close range to saturate the receiver and determine its compression point. This receiver's compression point was observed at $-20-\mathrm{dBm}$. Using the previously established $-100-\mathrm{dBm}$ receiver MDS an estimated $80-\mathrm{dB}$ dynamic range is found. These results are also very positive and support DAMS antenna pattern measurements that suggest a unidirectional radiation pattern.

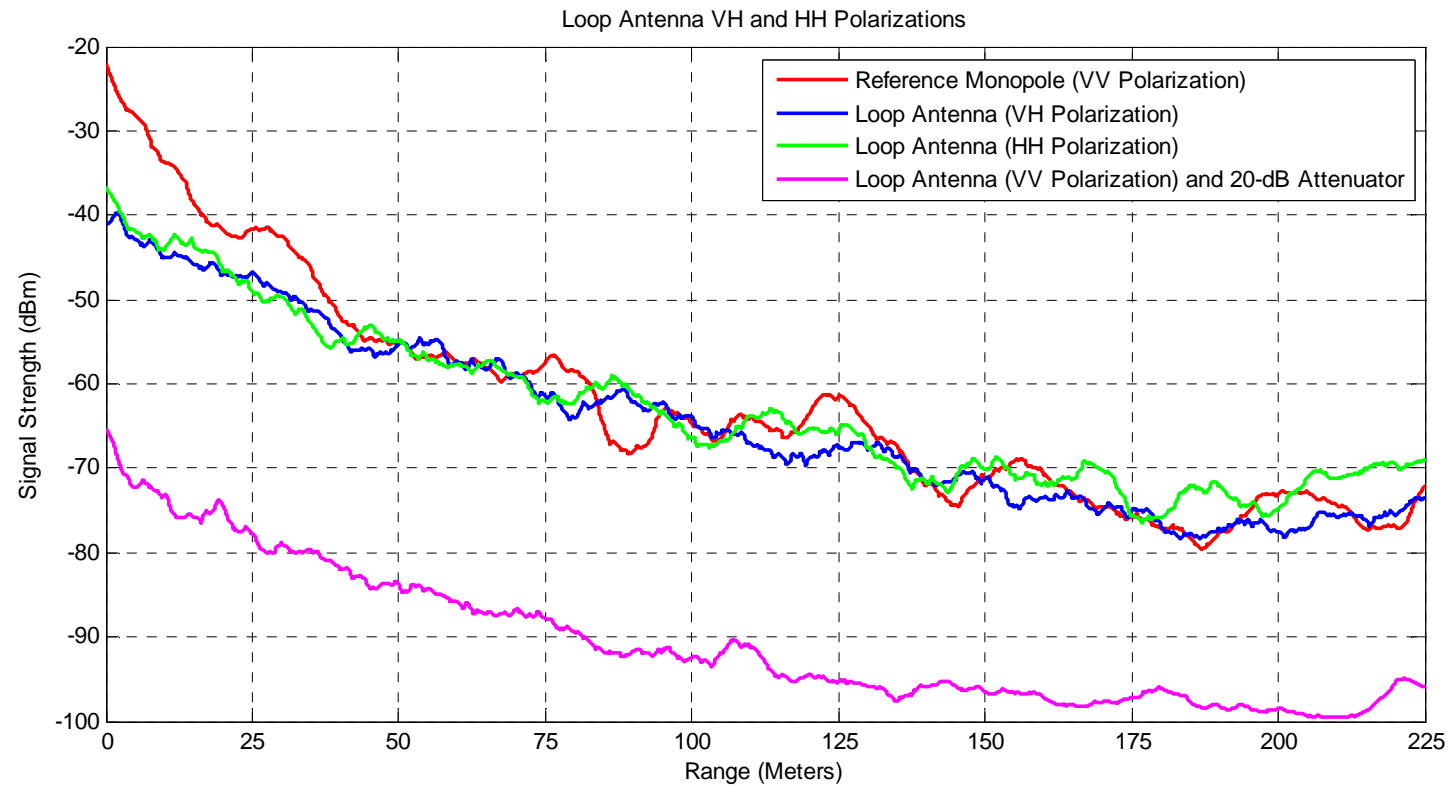

Figure 6.4.5: Plot of transmitted signal strength vs range for loop antenna polarizations. 


\section{Results}

The results obtained during live antenna testing show all antennas are capable of performing at the desired operational frequency with better than 10-dB return loss. The number of matching elements, operational bandwidth, S11 returns loss, and consumed PCB space is different for each antenna design and the radiation pattern of each design is heavily influenced by the effects of a non-ideal ground plane. These tradeoffs make employment of an exact antenna dependent on specific project requirements (e.g., a narrow operational bandwidth may be desirable for use in an extremely noisy environment). This section compares actual measurements to Ansoft simulation data, differences between the two are highlighted, and possible causes explaining the variation are offered.

There are both positive and negative aspects to each antenna design. The measured results and tradeoffs for each design are presented in the following paragraphs.

Due to inefficient layout arrangement, both the long and short meanderingmonopole antenna configurations can be eliminated for use in future designs. These antennas were not easily matched, consumed a large PCB area, and did not perform well with respect to S11 reflection coefficient at the desired frequency of $900 \mathrm{MHz}$. With the exception of these two antenna configurations, all other designs are able to achieve a $20-\mathrm{dB}$ return loss and minimum peak gain of $1.65 \mathrm{dBi}$.

The inverted-F antenna has a narrow bandwidth of $16.9 \mathrm{MHz}$, consumes very little PCB space, and requires no matching components because of its balanced feed line. This antenna is the second best performing design of all evaluated antennas. For the inverted-F antenna, the resonant frequency was already very close to 900 $\mathrm{MHz}$. All required tuning was achieved by simply trimming the radiating-element 
length. Due to the effect of a non-ideal ground plane, the antenna radiates uniformly in all directions making it highly versatile and incredibly useful in many situations. This antenna has a measured peak gain of $1.65 \mathrm{dBi}$ in the XY plane. The inverted-F antenna performs extremely well and the only drawback is its slightly lower return loss when compared to the loop antenna design.

The compact meandering-monopole antenna performs extremely well and is easily matched using a single 6.8 -nH series inductor. This antenna design is the third best performing design of all evaluated antennas. Its 78.4-MHz operational bandwidth is slightly less than the loop antenna's operational bandwidth. However, it does consume less PCB space than the loop antenna allowing for more component placement room. Its isotropic radiation pattern resembles a monopole and radiates equally in all directions making it highly versatile and incredibly useful in many situations. This antenna has a peak gain of $2.35 \mathrm{dBi}$ measured in the $\mathrm{XZ}$ plane. Its only drawback is a lower return loss when compared to the inverted-F and loop antenna designs.

The loop antenna achieves the best performance of all evaluated antenna designs. It is easily matched using a single $8.2-\mathrm{nH}$ series inductor. The loop antenna has the widest measured operational bandwidth at $84.4 \mathrm{MHz}$ and the highest return loss of $24.71 \mathrm{~dB}$. It has a peak gain of $2.73 \mathrm{dBi}$ measured in the $\mathrm{XZ}$ plane. The loop antenna was subjected to additional range testing due to its strong performance. Data collected during live antenna tests show a proven $20-\mathrm{dB}$ margin. Since range doubles for every $6 \mathrm{~dB}$ improvement in path loss, the loop antenna's maximum range can be calculated. Based on a 20-dB margin and Friis transmission formula, if the range contribution is allowed to increase while all other factors are held equal, we could expect a tenfold improvement for a maximum range of 2250 feet. Taking a more conservative approach, for a non line-of-sight indoor communication system, the range dependent loss is treated more like $1 / R^{3.5}$ instead of $1 / R^{2}$. Using this 
estimate, we can realistically expect to see a maximum range of approximately 838 feet. Similar to the compact antenna, the loop antenna's isotropic radiation pattern resembles that of a monopole radiating equally in all directions. This fact makes it highly versatile and incredibly useful in many situations.

A comparison of simulated data with respect to measured data is shown in Table 6.4-1. All data is compared after the addition of a matching network circuitry.

Table 6.4-1: Comparison of simulated and measured data.

\begin{tabular}{|c|c|c|c|c|c|c|}
\hline Design & $\begin{array}{l}\text { Simulated } \\
\text { Bandwidth } \\
\text { (2:1 VSWR) }\end{array}$ & $\begin{array}{c}\text { Actual } \\
\text { Bandwidth } \\
\text { (2:1 VSWR) }\end{array}$ & $\begin{array}{l}\text { Simulated S11 } \\
\text { Reflection } \\
\text { Coefficient at } \\
900 \mathrm{MHz}\end{array}$ & $\begin{array}{l}\text { Actual S11 } \\
\text { Reflection } \\
\text { Coefficient at } \\
900 \mathrm{MHz}\end{array}$ & $\begin{array}{l}\text { Simulated } \\
\text { Peak Gain } \\
\text { (dB) }\end{array}$ & $\begin{array}{c}\text { Actual } \\
\text { Peak Gain } \\
\text { (dB) }\end{array}$ \\
\hline & \multicolumn{2}{|c|}{ Larger value is better } & \multicolumn{2}{|c|}{ Smaller value is better } & \multicolumn{2}{|c|}{ Larger value is better } \\
\hline $\begin{array}{l}\text { Inverted-F } \\
\text { Antenna }\end{array}$ & $10.5 \mathrm{MHz}$ & $16.91 \mathrm{MHz}$ & $-30.0 \mathrm{~dB}$ & $-24.65 d B$ & $-13.9 \mathrm{dBi}$ & $1.65 \mathrm{dBi}$ \\
\hline $\begin{array}{l}\text { Long } \\
\text { Meandering } \\
\text { Monopole }\end{array}$ & $10.0 \mathrm{MHz}$ & $18.28 \mathrm{MHz}$ & $-16.8 d B$ & $-14.34 \mathrm{~dB}$ & $-13.8 \mathrm{dBi}$ & $2.4 \mathrm{dBi}$ \\
\hline $\begin{array}{l}\text { Short } \\
\text { Meandering } \\
\text { Monopole }\end{array}$ & $5.0 \mathrm{MHz}$ & $19.39 \mathrm{MHz}$ & $-14.0 \mathrm{~dB}$ & $-17.03 d B$ & $-11.2 \mathrm{dBi}$ & $1.29 \mathrm{dBi}$ \\
\hline $\begin{array}{l}\text { Compact } \\
\text { Meandering } \\
\text { Monopole }\end{array}$ & $7.0 \mathrm{MHz}$ & $78.46 \mathrm{MHz}$ & $-31.0 \mathrm{~dB}$ & $-20.44 d B$ & $-2.98 \mathrm{dBi}$ & $2.35 \mathrm{dBi}$ \\
\hline $\begin{array}{l}\text { Loop } \\
\text { Antenna }\end{array}$ & $7.0 \mathrm{MHz}$ & $84.48 \mathrm{MHz}$ & $-35.0 \mathrm{~dB}$ & $-24.71 d B$ & $-2.84 \mathrm{dBi}$ & $2.73 \mathrm{dBi}$ \\
\hline
\end{tabular}

Upon comparing the HFSS simulated data to the measured data several inconsistencies are noticed, particularly when comparing the peak gain values. There are many reasons possible why simulated results might vary from measured data, as previously discussed. For instance, the simulation model does not include the grounded coplanar waveguide feed, SMA connector, and is the matching network's lumped-element frequency-dependent loss due to resistance. Other simulation setup factors such as radiation boundary size and port stimulus dimensions also affect 
simulated data. The copper thickness after electroplating is thicker than the simulated $1 / 2 \mathrm{OZ}$ and is unevenly deposited over the sample creating an unpredictable thickness variation. The routing process creates jagged edges tapered to match the $\mathrm{V}$-shaped profile of the universal cutting tool; these lines were simulated as perfectly strait smooth edges and did not containing any taper in the simulated model. Even though the simulated numbers do not match exactly, simulated data being much lower than measured values, the actual trends in simulated data do follow suit with measured data. 


\section{Conclusions}

\subsection{Conclusions}

Two main objectives have been established: to compare the advantages and disadvantages of each type of antenna and to investigate the maximum attainable performance of these antennas in real world conditions. An additional requirement was established that a maximum range of 225 feet be attained with at least one antenna design. Based on the results shown, several conclusions can be made about the evaluated antennas. Each antenna design provides unique characteristics making each ideal in different circumstances.

The simulated data was able to point out which antennas would work well before implementation by comparing the expected return loss of each design. The simulated data accurately represented each antenna's resonant frequency and was able to accurately predict resonant frequency before matching circuitry. However, the actual complex impedance, operational bandwidth, and peak gain are different than simulated results. Overall, the measured antenna performance is much better than that predicted with the simulation. Several reasons have been provided for why simulated data may vary from measured data in the results section. HFSS does not have a good way to incorporate lumped element S-parameter data to account for frequency dependent matching network loss. The use of lumped RLC boundary conditions did not prove to be an effective method for predicting the expected matching network required to match each antenna to 50-Ohms. Even though simulated data did not match up exactly, overall data trends were useful in predicting the best overall choice.

Every design was able to be matched with a $2: 1$ VSWR and properly resonates at the desired $900-\mathrm{MHz}$ frequency. The inverted-F, compact meandering 
monopole, and loop antenna designs performed very well. These three designs have a measured bandwidth that is more promising than what is seen in simulated data. The compact meandering monopole and loop antennas are both wideband with at least 80$\mathrm{MHz}$ bandwidth, while the inverted-F antenna is relatively narrowband with $20-\mathrm{MHz}$ bandwidth. These antennas all have a very strong performance and were able to be well matched. Each design achieved an S11 reflection coefficient less than $-20 \mathrm{~dB}$. Depending on the desired radiation pattern and required bandwidth, any three of these designs would work well for an integrated transceiver design.

Problems were shown with the long and short meandering monopoles. These designs did not perform well with respect to performance of the other designs. This behavior is inherent in the folded monopole design and is caused by opposing currents traveling in opposite direction along parallel sections of antenna. The fieldcancellation effect caused in neighboring antenna elements reduces the usefulness and efficiency of the long and short meandering monopole antennas. In addition, the antenna footprint consumes over half of the PCB leaving very little room for added transceiver circuitry. These designs do achieve a 2:1 VSWR and properly resonate at $900 \mathrm{MHz}$, but have reduced efficiency when compared to the other designs, making them undesirable for integrated transceiver design.

The loop antenna was selected for additional range testing due to its strong performance when compared to the other antenna designs. The loop antenna was able to successfully transmit and receive over the full 225 -foot range with a demonstrated $20-\mathrm{dB}$ margin. This was proven using a $20-\mathrm{dB}$ attenuator between one antenna and its transceiver during range testing. In addition, it is estimated the loop antenna configuration has a maximum range of 838 feet. This range calculation was based on conservative estimates for spherical spreading loss. The omnidirectional radiation pattern makes the loop antenna highly useful and sought after for many different applications. Furthermore, due to the range testing results presented in Section 6.4, 
the antenna's performance is expected to be orientation independent. Measured results show the loop antenna performs very similar to the reference monopole antenna at distances greater than 50 feet. The loop antenna performance is exceptional when considering a printed antenna of its size.

Given a set of design constraints, the data collected and shared in this paper can be used to confidently select an electrically small printed antenna for transmission over a distance of 200 feet. Additionally, the antennas bandwidth and radiation pattern can be precisely chosen based meet specific product needs (e.g., a narrow operational bandwidth may be desirable for use in an extremely noisy environment). This knowledge helps to achieve more reliable product designs that work to their best of their ability every time. It has been shown that simulation software can offer a very good starting point for antenna design, significantly reducing the number of iterations needed for final product acceptance. However, it has been shown that simulation is not a suitable replacement to eliminate the antenna prototyping process. 


\section{References}

1. Bancroft, Randy. Microstrip and Printed Antenna Design: 2nd Edition. Raleigh, North Carolina : SciTech Publishing, Inc., 2009. pp. 1-9.

2. Chen, Zhi Ning. Antennas for Portable Devices. Sussex, England : John Wiley and Sons Ltd., 2007. pp. 24-25.

3. Pozar, David M. Microwave Engineering, Third Edition. Sussex, England : John Wiley and Sons, Inc., 2005. pp. 143-149,155-156.

4. Waterhouse, Rod. Printed Antennas for Wireless Communications. Sussex, England : John Wiley and Sons Ltd, 2007. pp. 183-184,258.

5. Bahl, I. J. and Trevedi, D. K. A Designers Guide to Microstrip Line. s.1. : Microwaves, 1977. pp. 174-182.

6. Edwards, T. C. Foundations For Microstrip Circuit Design. Sussex, England : John Wiley and Sons Ltd, 1980. p. 111.

7. Chia, Michael Y.W. and Chen, Zhi Ning. Broadband Planar Antennas: Design and Applications. Sussex, England : John Wiley and Sons Ltd, 2006. p. 135.

8. Wong, Kin Lu. Planar Antennas for Wireless Communications. Hoboken, New Jersey : John Wiley and Sons, Inc., 2003.

9. Bahl, I. J. and Bhartia, P. Microstrip Antennas. Dedham, Massachusets : Artech House, Inc., 1980. p. 3.

10. Associated Author. CC1100 Datasheet: Single-Chip Low Cost Low Power RFTransceiver. www.ti.com. [Online] May 2007. [Cited: February 2, 2009.]

http://focus.ti.com/docs/prod/folders/print/cc1100.html. p.21. 